$x+20 x+4$

3.th $2+x$

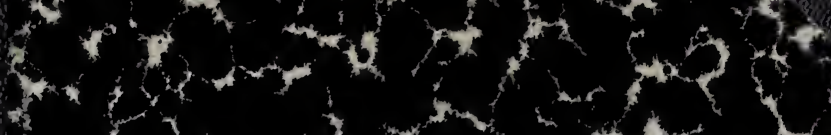
$x+2 x+2 x+3$

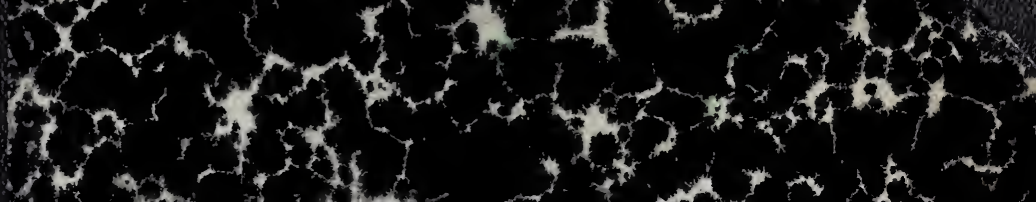

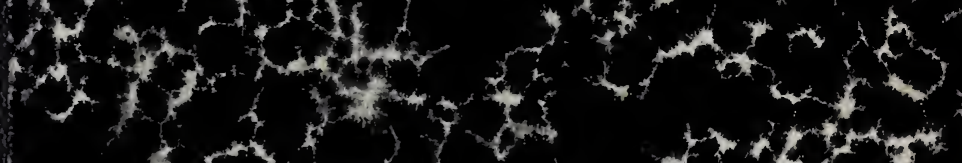

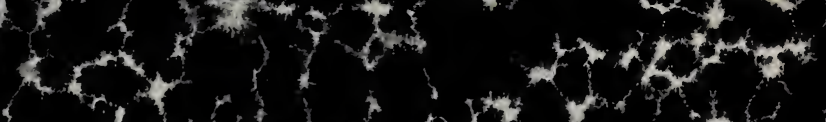
$x^{2}+x^{2}+x^{2}+y^{2}$ wat +4

$y_{2}+x^{x}+5$

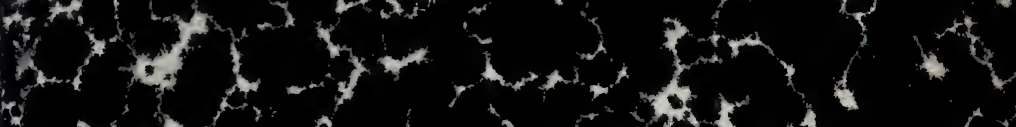
$\rightarrow+3+2 x$ $y-3, t^{2}+2 x$ at $x^{2}+x^{2}$ + $+4+2+1$ $6 y^{2}+2+2$ $\operatorname{coc}^{2}+x^{2}+x^{2}$ S) 3 lat $2 x x^{2}$, $y^{2}=10$

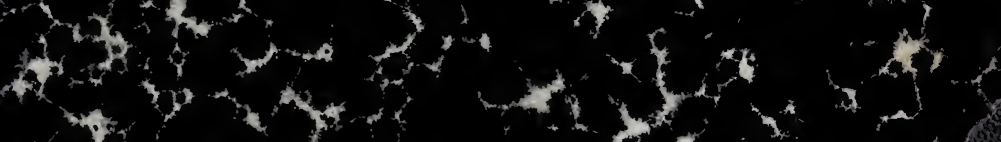
$-10+2+2$ ?.

tat $+2+2$ $x^{2}-y^{2}+4$ 


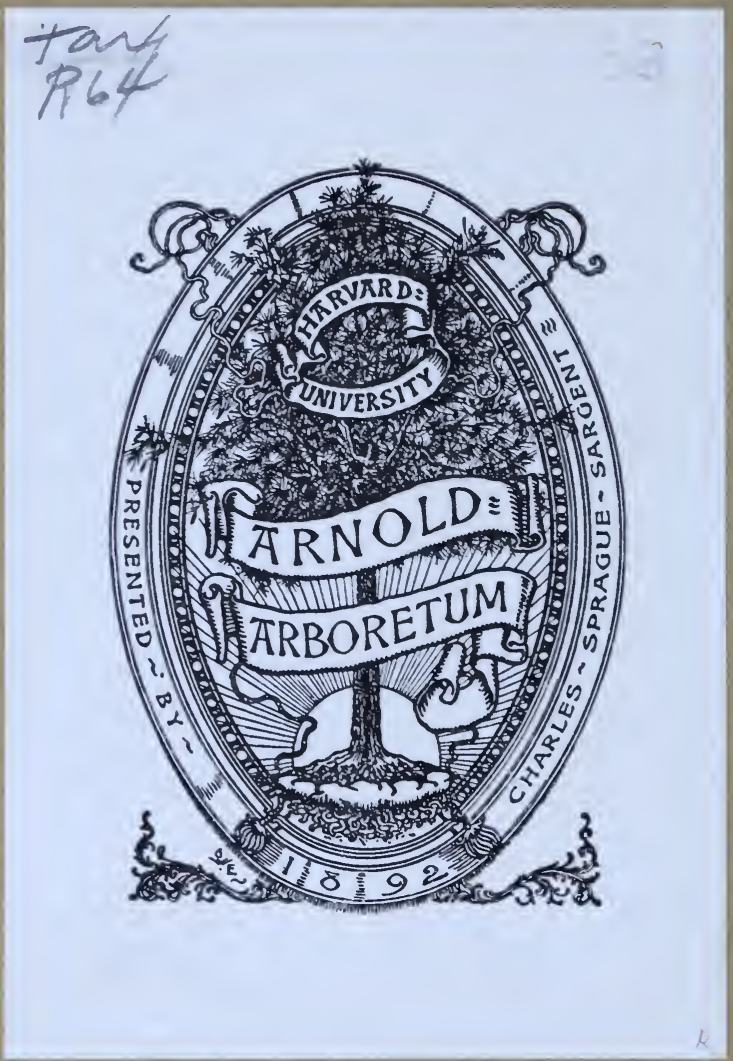






\title{
EugENe ROLLAND
}

\section{FLORE POPULAIRE}

$\cap \mathrm{U}$

\section{HISTOIRE NATURELLE DES PLANTES}

\author{
DANS LEURS RAPPORTS
}

AVEC LA LINGUISTIQUE ET LE FOLKLORE

$$
\text { TOME I }
$$

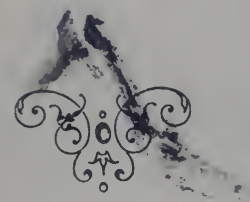

LIBRAIRIE ROLLAND

2, RUE DES CHANTIERS, PARIS 



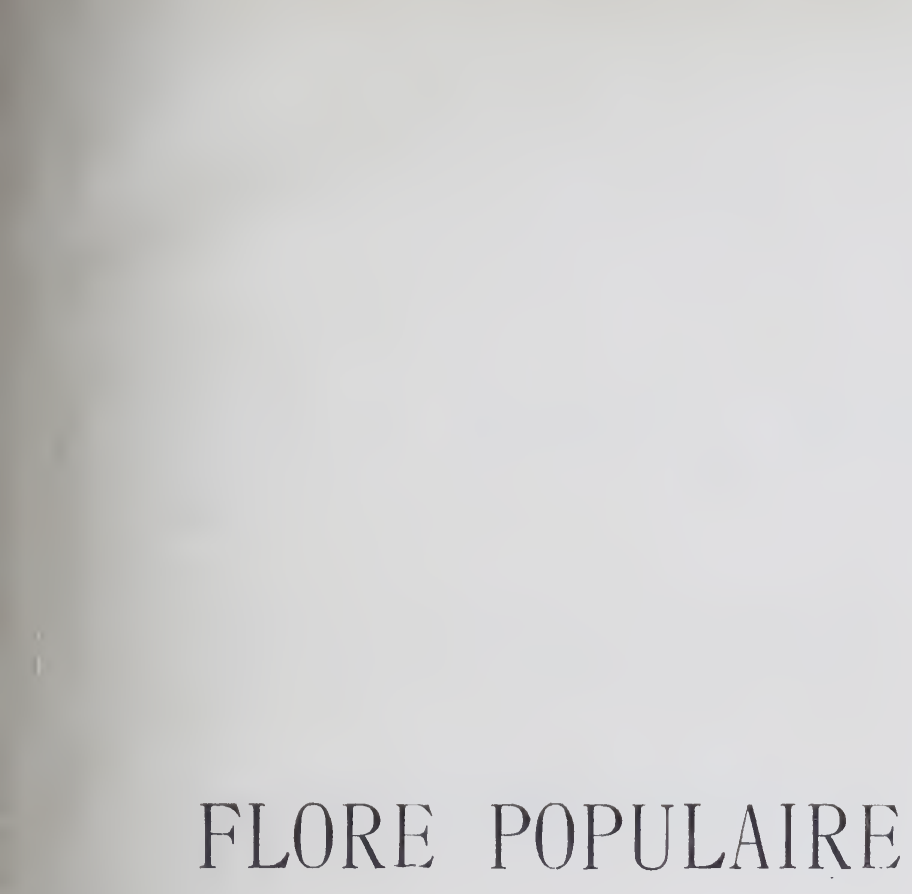

\section{FLORE POPULAIRE}


CHARTRES. - IMPRIMERIE GARNIER 
EUGENE ROLLAND

\title{
FLORE POPULAIRE or
}

\section{HISTOIRE NATURELLE DES PLANTES}

DANS LEURS RAPPORTS

AVEC LA LINGUISTIQLE ET LE FOLKLORE

\author{
TOMIE I
}

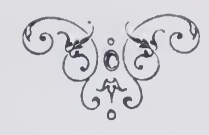

\section{LIBRAIRIE ROLLAND}

2, RUE DES CHANTIERS, PARIS 


\section{Woh.1911 23856}

Digitized by the Internet Archive in 2017 with funding from BHL-SIL-FEDLINK 


\section{INTRODUCTION}

Sous le titre de Flore populaire nous publions un recueil systẻmatique des noms populaires donnés aux régétaux et des proverbes, devinettes, contes et superstitions qui les concernent. Le domaine exploré, à res divers points de rue, est l'Europe ancienne et moderne, l'extrème nord de l'Afrique et l'Asie occidentale. Ie lecteur sapercerra bien vite que les diverses parties de ce vaste champ d'enquête sont très inégalement représentées dans notre Flore et que l'Europe occidentale y occupe une place prépondérante. C'est que, pour cette région, les sources nous ont été plus facilement accessibles.

Les documents que nous avons mis a contribution sont les suirants :

10 Les ouvrages imprimés de toutes sortes, principalement les Vocabulaires des divers idiomes et les Flores locales. 10 n trouvera la liste des auteurs cités à la fin de chaque volume.

$2^{\circ}$ Ceux que nous avons été chercher nous-mème dans ce puits sans fond qu'on appelle la Tradition orale. Ils sont suivis des mots : recueilli personnellement ou, en abrégé, rec. pers.

30 Ceux que nous derons à l'obligeante communication de nos amis et correspondants. Ils sont suivis chaque fois de la mention du nom de la personne qui a bien roulu nous les envoyer. 
4. Enfin ceux que divers savants ont extraits pour nous d'ouvrages qui pour ètre utilisés, demandaient une compétence spéciale. lls sont suivis des initiales de leurs noms mises entre crochets.

Voici la liste de ces personnes, que nous tenons à remercier d'une façon toute particulière:

II. H. GAlboz, pour les langues celtiques (moins le loreton). -

M. E. Ensivit, pour le breton. Abréviation : [H. G.]

I. A. DE Conk, pour le néerlandais thollandais et llamand.

M. H.-F. FeILberici, pour les langues scindinaves.

M. TH. Volkor, pour les langues slares.

11. A. Gorover, pour le roumain.

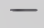

[E. E.

II. J. Vinson, pour le basque.

II. Eir. Lalakaxtz, pour l'arménien.

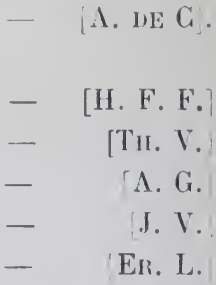

MM. J. Gayus J. ( . En. Enmont En. E.), et feu H. JenssenTrscil [11. J.-T.] pour divers noms de plantes relevés dans des ourrages de botanique.

Nous avons classé les plantes dans l'ordre généralement adopté par les botanistes modernes, c'est-à-dire que nous avons suivi pour les Familles la clissification de 1.-P. DE GANdolle (Théoric de la

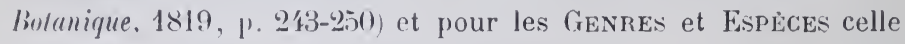
de Nrmax (Sylloge florar puropeae, 1854-185̃).

Nous arons 'lissé les langues et les dialectes dans l'ordre suivant:

Grec ancien. grec du moven-âge, grec moderne.

Latin ancien, latin du moyen-îge.

Nomenclature latine savante de la Renaissance et de nos jours. Français ancien et moderne, dialectes et patois gallo-romans (de la France, de la Suisse romande, du Pays wallon, des Iles Normandes, du Val d'Aoster.

Italien, ladin.

Espagnol. 
Portugais.

Catalan.

Roumain.

Allemand.

Néerlandais |flamand et hollandais।.

Anglais.

Langues celtiques.

Langues scandinaves.

Langues slaves.

Langues lithuaniennes (lithuanien et letton.

Albanais.

Tsigane.

Basyue.

Magyar, esthonien, finnois.

Arabe, persan, ture, arménien et autres langues orientales.

Nous terminons en faisant appel it de nouveaux collaborateurs pour les volumes suivants de la Flore populaire.

Eugène Rolland.

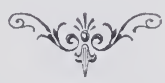





\title{
FLORE POPULAIRE
}

\section{RENONGULACEES}

\author{
ATRAGENE (') ALPINA. (LINEE, 1753) \\ NONS DE CETTE PLANTE.
}

clematis alpna geranifolia, nomenclature savante, C. Batni, Prodromos theatri botanici, Basil. 1671, p. 135.

clematis cruciata alpina, nomencl. sav., PONA cité par BAuHix, Pinax, 1671, p. 300.

clemalis alpina, nomencl. sa\%, Miller, Dicl. 1768.

vilalbina dei sassi, italien, Nomi adoperali. 1873.

vabies, Mont Cenis, J. F. RE, Flor'a segusiensis. 1805.

curpenitca, napretsnic, roumain, BRANDzA.

naprasnica, roumain, Сıнас.

doppelblume, allemand.

umwund, Altenau, Pritzel al Jessex.

yoasstrauben, bavarois, ScHMELLER.

srobotina, serbo-croate, Šulek.

dikoï chmiel, russe, Falk, Beytr. 1786.

zurganysch, tatare, FALk, Beytr. 1786.

\section{CLEMATIS VITALBA. LINNE - LA CLEMATITE DES HAIES.}

1. NOMS DE CETTE PLANTE.

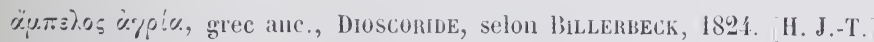

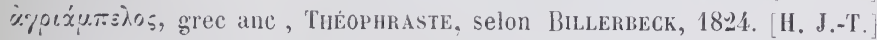

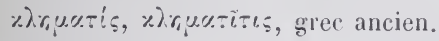

xinurs:id\%, grec mod., Portu's, Diclionarium gr. barb. 1635.

clematis, latin, PLINe, Hist. nat.

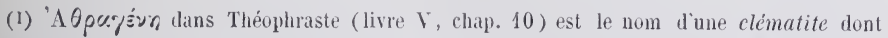
lidentification n'est pas très certaine. Linné a eu le tort de ne pas écrire athragene comme l'avaient fait ses prédécesseurs Anguillara, Césalpin, J. Ray. (Voy. à ce sujet A. Gras, dans Bull. de la Soc. bot., 1860, p. 907). 
clematis, clematitis, clematitis syluestris, clematitis sylvestris latifolia, vitis sylvestris, vitis nigra, vitalba, viburnum yallorum, atragene Theoplrasti, vitis syleestris caustica, viorna, nomencl. sav. du Moyen-Age et de la Renaissance, Buunis, Pinax. 1671.

clématite, f., clématite des haies, f., clematite grimpante, f., français.

clématile, $\mathrm{f} .$, français populaire.

vidalbo, f., Toulouse, AzAïs, Dict. des illiomes rom.

bidalbo, f., Hérault. - Aveyron. - Haute-Garonne. - Lot. - Tarn. -

Tarn-et-Garonne.

vidaouba, f., Tulle (Corrèze), G. de Lépinar, Noms.

vidaoubo, f., Corrèze. - Gard.

guidaoubo, f., Brive et UsseI (Corrèze), G. DE LÉPINAr, Noms.

bidaoua, f., bidaouèrés, f. pl., Luchon (Pyr.), comm. par feu J. SAcaze. bidaouo, f., env. de Moissac (Tarn-et-Gar.), rec. personnell.

bidablé, f., Haute-Garoune, Poumarède. - Montauban, Gaterau.

guidalbé, f., Périgord, Poésies popul. de la France, Mss. de la Bibl. nat., f. fr., 3341, fo 147.

bidlaoude, f., Tarn-et-Garonne, ADriex PAgès, p. 193.

bidaoucle, f., Landes, MÉTIVIER. 1839 ; et comm. de M. J. DE LAPORTERIE. bidaouclo, f., sud-ouest du Lot-et-Garonne, comm. par M. l'abbé L. DARDI. Gers, comm. par M. H. Daignestous.

billaoule, f., bidaoulere, f. Armagnac, comm. par M. J. dE LAPorterie. bidaou, Gironde, LATERRADE, 1846. [H. J.-T.].

billaouguero, f., gascon, IzAïs. Dict.

biraougo, f., Lot, Puel, Catal. des $p l$.

birnoügneros, f. pl., gascon, Guide des Gascons, Tarbes, 1858.

bilaryo, f., Saint-Germain (Lot), Sovlié.

visable, f., Isère, A. Gras, Hist. nat., (dans Statist. de l'Isere, II. 1846). visaube, f., Saintonge, Jôsaix; Boucherie, Patois.

risoucille, f., Corniéville (Meuse), comm. par M. F. Bохnardot.

viablè, f., canton d'Aime (Savoie), comm. par M. Marjollet.

yable, f., envir. d'Albertville (Savoie), rec. pers.

rouable, f., Genève, Beadquer, Dict. du Doubs, 1881.

ourable, f., Sallanches (Savoie), rec. pers.

l'ouablla, f., Suisse romande, BrIDEL, Glossaire.

wouaublla, f., Vallorbes (Suisse rom.), Vallotton-Aubert.

voublè, f., La Salle (Val d'Loste), rec. pers.

wabthe, f., (avec th doux anglais), Bas Valais, Gilliéron.

vouailles, f. pl., Jura, rec. pers.

vouaille, f., Doubs, Beadquer, Vocab. du Doubs.

veilleee, Franche-Comté, Perrox, Proverbes de la Fr.-Comté, 1876. 
vélier, m., véhier, m., éveuillie, m., Doubs, BeAdquler, Voc. du Doubs. villé, m., Forèt de Clairvaux (Iube), Bacdoun, Patois de la Forêt de Clairv. véillie, m., rouâiè, m., bois à la vieille, m., bois à la vigne, m., Franche Comté, DART01s.

vélie, m., véïe, m., Montbéliard, Contejeax, Gloss. du pat. de M. veuillet, m., viieux, m., viée, f., viie, f., Hte-Marne, c. par A. Daguin. vieille, f., Tranqueville (Vosges), Halluaxt, Flore pop. des Vosges.

vellhana, f., Suisse romande, Bridel, Gloss. du pat. de la Suisse rom. valiniè, m., provençal mod., Garcis, Nouv. dict. provençal, 18.11. vioche, f., Anjou, Desvatx, 1827. - Deux-Sèvres, comm. par I. B.

Souché. - Chef-Boutonne (Deux-Sèvres), Beacchet-Filleat. viouche, f., Deux-Sèrres, comm. par M. B. Socché. vioche blanche, f., Charente-Inférieure, comm. par M. E. LEMarié. virgouenne, f., vigouenne, f., vigougne, f., Centre de la France, Jaupert. vigane, f., vicane, f., Centre, Jaubert.

vigonne, f., Ineuil (́canton de Châteauneuf, Cher), rec. pers. vis, Meuse, Iovisr, Hist. nat. de la Meuse, 1835. vissano, f., arissano, f., Gard, Rev. des lany. rom., 1884, p. 70. - Anduze

(Gard), Viguier.

ravisano, f., Languedoc, SAUVAges, Dictionn.

bissana, f., Montpellier, LoRet, Filore.

vigne sauraye, f., Mayenne. - Ille-et-Vilaine.

vifranche, f. Villers-le-Sec Haute-Saòne), rec. pers.

folle vigne, f., fausse vigne, Manche et Calvados, Joret.

vigne de la Vierge, f., Envir. de Caen, Joret.

vigne blanche, f., aubevigne, f., français de diverses provinces.

aoubavit, f., Var, Mistral.

aoubovit, f., Gard, c. par H. P. Fesnuet.

aoubavis, f., Var, Hanry. - Porquerolles, OLlivier.

abravis, f., Marseille, Mistral.

aoubujo, f., Alpes, Mistral.

aoubugea, f., Digne, Hovnorat.

obi, m.. Centre de la France, Jacbert, Gloss.

obisse, Nivernais, c. par M. A. Mlllies.

vigno de Salamoun, f., provenc. mod., Mistral.

vigne du diable, f., Eure-et-Loir, c. par M. J. P0QUET.

viorne $\left({ }^{1}\right)$, f., français ancien et mod. - Normandie. - Orléanais. -

Anjou. - Berry. - Poitou. - Champagne. - Nivernais. - Bourgogne. vionrna, f., Basse-Auvergne, Malval.

(1) Du mot latin viburnum, qui plus ordinairement, s’applique à d’autres plantes. 
viorne des paurres, f., français, NEмxicH, Catholicon.

vienne, f., grande vrône, f., Anjou, Desvaux, Flore.

grosse vrone, f., Mayenne, comm. par un botaniste de la Mayenne.

viône, $f$., Mayenne, IoEm. - Normandie, Joret.

vianne, f., Berry, Janet-Massicault, Thibaul, roman berrichon, 1870 .

viennes, f. pl., Centre de la France, Jaubert, Gloss. du Centre.

viène, f.. Vendée, Lalanwe, Gloss. ilu pat. poit.

vieyne, f., Pays messin, D. LoRraix, Gloss. du pat. lorrain.

vêne, f., Pays messin., rec. personnell.

jorne, f., pays d'Ouche (Normandie), Joret.

yorne, f., Annoville (Manche), comm. par M. J. Couraye du PARc.

liorne, f., liône, f., liane (', f., nor'mand, Jonet.

vianchi, f., Villefranche (Rhòne), I'Uitspele.

lianchi, f., lyonnais, Purtspele.

liébos, m:, Issoire (Puy-de-Dòme). c. par M. J. Bareire.

tortile, f., Valenciennes, Hécart.

redorta, f., Montpellier, Lonet, Flore de Montp.

redouorto, f., Apt (Vaucluse', Coligxos, Flore d'Apt.

ridorte, f., Pyrénées-0rientales, Compario.Ilist. nat. des Pyrénées Orient. regourtiol (2). m., Aveyron, VAissier, Dict. patois de l'Aveyron.

viorte, f., français.

rampille, f., Haute-Normandie, Delboule; Joret.

rampioule, f., wallon, GRANdGagNaE. - Spa, LEzAACK.

gripette, f., wallon, Felder.

traineau, m., Centre de la France, Bonesu. Flore du centre.

trainiau, m., Centre, Jaubert.

trainasse. f., Morvan, Chambere, Gloss. du Morv.

tréna, m., Plancher-les-Mines (Haute-Saône), Poulet.

entrevalis, m., Arles, Laugier de Chartrouse, 1838. - Bouches-du-Rhône,

Villexeute. - Aix-en-Provence, Boyer de Foxscolonbe. - Var,

A.HC. -- Euvirons d'Avignon, Palux.

entrevigé, 11., Gard, comm. par M. P. Fes@uet ; - Polzolz.

entrevedil, m., Gard, Porzolz, Flore, 1857.

anbriri, f., Létra (Rhône), Сhabert, Patois de Létra (dans Revue des patois, 1888).

(1) Ces muts viennent de viome derenu yorne avec laarticle $l$ soudé protéthiquement. Voila prubahlement l'origine du mot liane qui aura été transporté en Amérique où il a ser vi à désigner diverses plantes sarmenteuses et grimpantes.

(2) En patois cérenol regourtilha a le sens d'entortiller, enrouler (AzAïs, Dict. des idiomes du midi). 
beligris, m., beligasso, f., birgasso, f., Aveyron, Varssier.

coulmon, m., Allier, comm. par M. C. Bourgougnon.

sariejo, f., Gard, comm. par M. C. Feseuet.

sariuéjho, f., languedocien, SAuvages.

sain bois (1), m.. Centre de la France, Jaubert.

sén-buas, m., Ile d'Elle (Vendér), Revue de Philol. frane', 1889, p. 117.

bois-garou, m., Nivernais, c. par M. A. MnLliex.

houblon, m., hoblon, m., Hante-Marne, comm. par A. DaguiN.

liseron $\left({ }^{2}\right), \mathrm{m}$. français du XVIe siccle, Livocier Mist. des Plantes, 158.4.

- Matthiole, Commentaires, 1655.

drabieux, Côte Saint-André (Isère), Robrs, Essai de topographie médicale de la Cöte-Saint-André, Lyon, 1855.

bois de bique, m., Haute-Narne, comm. par II. A. DAguis.

coune de gaïsse, f., (= corne de chèrre', Retonfey Pays messin), c. par

M. Iuricoste de Lazaronce.

jussemi de borro, (c’est-ì-d. jasmin d’àne), Pyrénées-0rientales, Companro. jaoussemi dés azés, m., erbo del vent. f., Gard, comm. par M. P. Fesquet. range, Verviers, Lejeune.

rang, Spa, LEzAגCK.

ranteh, wallon, FELLER.

herbe aux yueux, f., trancais.

yèp' du bribeu f., (herhe du mendiant), Sјa, LEZAACK.

barbe d's chipuve, f., Centre, Jaubert.

barbe de chievre, f., Anjou. Drswarx. - Vormandie, Joret.

barbe au bon Dieu, f., barbe du bon Dieu, f., Nomandie. - Champagne barbe de Judas. f., Maine, Desportes. H. J.-T.].

barbe de capucin, f., Aube, Des Etangs.

barbubuto, f., Alpes, Mistril, Tres. d. Fel.

cheveux de la Vierge, m. pl., Borry. - Champagne. - Dauphiné.

chevenx de la Bonne-Dame. m. pl., barbe de Totre-Seigneur, f., Centre de la France, Jalnent, Giloss.

traut de chin, (e.-it-d. laisse, corde, trait de ehien), (iendreville (Vosges)

et Virecourt (Meurthe), Hallant, Fl. pop. des $\mathrm{I}$.

bois ì fumer, m., Normandie. - Champagne.

bois de fume, m, Wignehies, prè Ivesues (Ford).

(1) Cette plante est ainsi appelée parce qu'elle peut remplacer, at point de vue médical, le veritable sain bois, Daphne Gnidium. - Jaubert.

(2) La figure représentée par Linocier suus le nom de liseron est hien une clématite et non pas un convolvulus. Est-ce par suile diune errenr? - Comprarez le mot lisoni, nom vénitien de la clematis flammula. 
bô fumant, m., bô fumâ, m., Vosges, Haillaxt, Flore.

bois fumerot, m., Doubs, BeatQcier. - Montbéliard, Conteseas.

bô femerot, m., Montbéliard, Conteseax, Git. du pat. de $I$.

fumerotte, f., Doubs, Beavquier, Voc. du Doubs.

fumailles, f. pl., Salins (Jura), rec. pers.

fumei, m., environs de Cambrai, rec. pers.

feum bo, m., bouè d' toúbali, m., bo d' toubali, m., wallon, Fruler.

bois de pipe, m., herbe de pipe, f., Haute-llarne, comm. par 1. D.aum.

djon, tournan-bouè (bois tournant), m., wallon, FELLER.

cranliegni, m., Somme, Ledied.

crinquillier, ut. Samer (Pas-de-Calais), r. par II. B. dE Kenuervé.

crinkiyie, f., incrinque-hayure, f., Saint-Pol (Pas-de-Calais), com. par

II. Ed. Eusoxt.

cranquillure, f., Manin (Pas-de-Cal.), com. par II. Ed. Evм0یx .

crinquillure, f., crinque-hayure, f., Ramecourl (Pas-de-Calais, com.

par M. Ed. ЕDмo.т.

diable en haie, m., Manche, Joret.

vitalba, clematide, italien.

fior di mimuè, ital., Targioni, Diz. botanico, 1825.

capponi, m. pl., (les fruits cotonneux de la plante), T.ragiosl, Di: botanico. vis saruaja, piémontais, Annales de l'0bserv. de Turin, 1811, 1. 14.1.

viorna, piémontais, Balbis, Piante dei contorni di Torino. Torino, an IX.

- Zalli, Dis. piem.

vitalbera, visrabbia, Turin, Nomi adoperati.

liarba, piémontais, ZALl, Diz. piem.

bialba. mialba, rialba, vialba, Vérone, Pollixi, Flora veronensis, 1892.

vialber, erba der pitocch, milanąis, BAxf, Voc. mil.

ridárbola, Còme, Moxti,. - Brescia, Melchiori.

viderbola, Plaisance, Nomi adop.

videtbor'a, Milan et Côme, Nomi adop.

vidalbora, Côme, Comolli. [H. J.-T.].

vinèrbola, caurixula, ligabosch, milanais, Cherubisi.

videlbour, Pavie, MANFred.

vidalba, bolonais, Coronedi-Berti. - Mantoue, Cherubini.

vilèlba, Modène, Parme, Plaisance, Nom adop. - romagnnol, Morri.

ritabia, Rome, Pérouse, Nomi adop.

ritabbia, Env. de Pérouse, Zaxetru, p. 203.

vitarba, sicile, Cupaxi, Hortus catholicus, 1696; Nomi adop., 1873.

visalba, Pérousc. [H. J.-T.].

vialba, Vérone, Pollini. [H. J.-T.].

inalba, Valle Imagna, près de Bergame, TiRABoschi, 1873. [H. J.-T.]. 
inambolı, Valle San Martino, près de Bergame, Tiraboschi. [H. J.-T.].

viturbo, Messine, Nomi adop.

viturrole, Calabre, Pasncale et Avelliwo.

vitarva, Cosenza, Catanzaro, Nomi adoperati.

villasa, idasa, Brescia, MelchioRI.

vidison, Vicence, Nomi adoperati.

i'ileson, Trévise, SACcardo.

vidigarle, Trévise, Nomi adoperati.

vidercia, Parme, Malaspixa.

vit bastarda, eua de bosch, Bergane, Nomi adoperati.

vite falsa, Forli, Ravenne. Ferrare, Bologne. H. J.-T.!.

vida salvarlga, Parme, PEschien, 1828. 11. J.-T. .

viticella, vitaglia, Avellino, Nomi adoperati.

viticedda, sicilien, Mortillaro.

vitaura, lschia, Gussoxe. 1854. H. J.-T.].

vidizela, Brescia, МЕцсн1юn, 1817. [Н. J.-T.]

iræèr, Valle Gandino (pris de Bergame, Tiraboscin. [H. J.-T.].

veticchia, vilicchia, vitricchia, vitjielva, vita selvatica. Abruzzes, Finduore,

rocabulario dell' uso abruzzese.

viticchio, Aquila et Ascoli-Piceno, Nomi adop.

viticchie, Ancône, Nomi adoperatı.

vitosa. Sud de l'Italie, Nomi adop.

vitacchiosa, Chieti, Tomi adop.

vitoja, vilalbo, vitacchia, Potenza, Nomi adop.

vitajo, Bari, Nomi adoperati.

grambollino, Cosenza, Tomi adop.

vitusara, vitusa, Catanzaro. Nomi adoperati, 1873.

vilaja, tortavitaja, Vésuve, Pasncale, Flora vesuriaua, 1869.

vitara, vitrara, Naples, PAsqCAle el Arellixo. Flora medica di Napoli, 18 il vilura, vilusara, Reggio (Calabria), Nomi adoperati.

ligabosch, reabla, Brescia, Nomi adop.

ligara, Mirto Sicile), Crpsir, Hortus catholicus, 1696. - Sicile, Nomi adoperati, 1873.

ligauu, Sicile, CrрAxi, IIot'. cathol., 1696.

ligonia, Calabre, PAsq[ALE, 1811. [H. J.-T.].

passionén, Parme, Malaspixa.

gusserna, Parme et Plaisance, Nomi adoperati.

yuz:eller, Reggio, Nomi adoperati.

eibu dei censiosi $\left({ }^{1}\right)$, italien, d'après Sidxo.

(1) Dicesi in Toscana erba dei censiosi, perche i poveri applicano sulla cute le foglie 
clemati, Gênes, Nomi adop.

sciarboa, Gênes, Cas.acciı, Jicr. genorese.

blaudin, blaudinarie, brundinarie, Frioul, P'ıroxa, Vocabolario friulano, in $-8^{\circ}, 1871$.

ridigada, guiz, roaje, vidigon, ridison, viligada, r.zgoleze, Belluno, Nomi adoperati.

bensigliu, zara, Sardaigne du sud, MoRis, Flora sardoa, 1837-185\%. auzzơra, aะzara, zara, Sardaigne méridionale, Sraio.

isterzu, stersu, Lanussci (Sardaigne), Moris, Flora sardoa, in-10, 1837-1859.

auciada, Iglesias (Surdaigue), Monis, Fl. sarloa, iı-4, 1837-1859!.

bidighinsu, Cuglieri (Sarlaigne), Moris, Fl. sardoa.

vitichingiu, Fonni Sardaiguej, Mloris, Fl. sarloa.

idrighinzu, sarde logodourien, SPaxo.

ridalba, muermo, muermera, yerba del pordiosero, vid blancu, espagnol. erba d'os pordioseiros, erba d'os mendigos, erba d'o póbre, gallicien,

VAlladares.

rillauba, catalan.

vidalba, ridorla, Vall de Nuria, Varreds.

viadella, Calella (Catalogne), Cixi, Flora de Calella (dans Anuuri de la Associacio d'excur's. calalana, 1883.

vidaura, vidarsa, ratalan, CoLyerro, 18.6. [H. J -T.].

vidriella, Valence, CoLmeiro, 1846. [H. J.-T.].

viligera, Aragon, Willkome et Laxe. [H. J.-T.].

virgaza, virgaraza, Santander, Comission, 18\%. [H. J.-T.].

botiguerus, enredadera, Aragon. Comission, 1872. Н. Ј.-Т.].

erba de llagos, manaiula, catalan, CoLMerro, 18.16. H. J.-T.]

nue:za, nue :za blanca, rastillan, Colmerro, 1846. H. J.-Т.:

vide branca, sipo de reino, portugais.

mormeiru, gallicien, Curf.ro, Vicc. gallego.

luminosa, vitga alba, roumain, Braxozs, Limba hotamica.

curpen, liurpen, roumain, CiнAc, Dict. daco-rom. - roumain transylva-

nien, Fes\%.

waldrebe, brennliraut, brennu'ur, allemand.

liela, liola, hranlia, anc. liaut allemand, Dıеғехвасн, Gtossarium.

bettlerseil, allemand, Grim, Deutsches Woert.

u'aldstricli, allemand, Grassinsx, Deutsche I'flam:ennamen, 1870.

tockeburt, bockishart, düels-tweren, Gattingue, Pritzel et Jessex. 
gänsemord, Alsace.

judesel, Luxemb., KoLtz, Flore du Grand duché de Lux. 1873.

lelleliken, Luxembourg, J. HEBER.

lehnhech, Eifel, Wintgen, Tegetalion der Eifel, 1865.

liene, Autriche allemande, Pritzel et Jessex.

teufelsreben, Carinthie, ZwaxzigER.

niala, raücherli, canton de Saint-Gall Suisse), Wartjidxx.

petersbart, teufelszwirn, Golling, Pritzer et Jessex.

helikenläım, f., lurenseil, n., Souabe, Scнмш.

lïhlen, allemand de Transylranic, Fusz.

lirlisch, Merkersdorf (Basse-Autriche), comm. par M. F. Br.sky.

nursche, nirschn, lirisch, lirchn, jüln, lirlacher, lirchliozll, lurschn.

lïlyen, liarlisch, jullische widn, ulawiedn, ulischwidn, hatl'n $(=$ les

fruits de la pl.), Autriche all, , F. H(EFER.

lynen, flamand, Dodoxaeus, 1644; OUdeMass, 1872. [А. DE CoCK].

heglioorile, klimkoorde, flamand. [А. DE Cock.]

heete lilim, flamand, Rocces, Flore du Nord de la France, 1803.

clementine, flamand, DE Bo, Westrlaamsch idiotikon.

virgin's bower, wild clematis, climber, anglais.

traveller's joy '1), anghlais, Cotgrate, F'ench. dict., 1650.

smolie-urood, anglais, Prion, Popular names of british plants, 1870.

grey beards, old man's beard, old man, smoking cane, Devonshire, Friend.

old man's woozatl, lady's bower, begyar-brushes, maideu's honesty,

honesty, honey-stick, love, love bind, bullbind, bellywind, bedwine,

bethwine, bindwith, hay-rope, crocodile, dans les difl. dial. de

l'Augret., Britt, et Holl, 1878.

lus-slreapach ( = plante grimpante, gaélique d'Ecosse, MaC ILPINE et autres H. G.?

ysyarllys bychan $i=$ la petite ysyarllys; ysgarllys = la plante qui se divise), gallois, J. Daries, 1632 et J. Walters, 1794. [H. G.?

barf y ywr hen = barbe: du vieillard), cudd y coed (= qui cache le bois),

gilllois, Hlugir D.ties, 1813. [H. G.].

dringiedydd = grimpeur), gallois, S. Evass, 1852. E. E.].

roüanés (= reine), breton, P. GrégoIRE de Rostrexen. [E. E.].

rouallez, breton, Le Goxidec, Troude, LIÉgand. [E. E] ].

barvinek, tchèque, A. Mubller, Alph. Warterb. synonymer Namen der offic. Pflanien, l'rag, 1866.

tita, tilul, lom, biela loza, le $a$, serbo-croate, ŠLeK.

(1) Ainsi appelée parce que le voyageur recunuait qüil est arrive auprès l'un village lorsqu il voit les haies couvertes de rette plante. 
borodarnyl (plante des verrues (1)), petit russien [Tн. V.]. aihen zuri, basque, LACoIzQretA, 1888, [J. V.] s:ulak, magyar, Niklosicir, Slav. Elem. im mag. p. 117. fejer renit:, magyar, Fusz, Trivialnamen d. Pflan:en in Siebenburgen. melaa', arahe du Mont-Liban, Berggrex, Guide francais-arabe, 184. tschermaluk, tatare criméen, Stevex, ler:. d. taurischen Pflanien. tschermau'uk, tatare criméen, Pallas, Observations faites dans un voyaye en $1793-94$, II, 393.

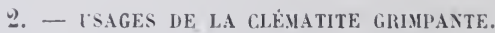

Ist iı allen Theilen brennend scharf, so dass sie auf der Haut leicht Blasen und Geschwiire lerrorruft. Man hat das liraut, Herba et Stipites Clematidis sylvestris bei syphilitischen nnd rlemmatischen Zustænden, Wassersucht, Wechselfieber, ansserliclı gegen lirelze und Geschwïre, die Wurzel als Abfiihrmittel, in frïherer Zeit auch das zerquetschte Krant als Vesikator angewendet. Die Triebe dienen in Slavonien zur Anfertigung von Bienenstocken, Korben n. dgl. - Rosextnul, Synopsis plantarum.

Toutes les parties de la clématite des laaies ont une saveur àcre et brùlante. Ses feuilles fraiches écrasées et appliquées sur la peau, rougissant d'ahord lit partie avec laquelle elles sont en contact, y font naître de l'inflammation, y produisent des vessies, et par snite des ulcères superficiels. C'est de lii que cette plante a recu le nom d'herbe aux gueux, parce que certains mendiants s'en servent pour se fuire renir des ulcérations aux bras ou aux jambes, et par là exciter la commisération. Ces ulcères ont peu de profondeur, sont larges à volonté, et se guérissent facilement: il suffit de les couvrir avec des feuilles de poirée, et de les garantir du contacl de l'air.

Les paysans en font un cautère, ils prennent la fine pellicule qui est sous l'écorce, la roulent en forme de pois et se l'appliquent sous un sou fortement comprimé par une bande. Le trou se forme aussi hien quavec un caustique plus puissant. - Nirernais, c. par M. A. Millien.

La cuisson dans l'eau fait perdre à ses jeunes pousses leur ìcreté, et dans quelques parties de l'Italie, principalement dans l'État de Gênes et dans la Toscane, les gens de la campagne les mangent après les avoir ainsi próparées $\left({ }^{2}\right)$. En Provence les paysans se servent de la clématite quand elle est

(1) Il vient des verrues aux mains à celui qui fuuche cette plante. [TH. V.]

(*) Dans le département de l'Hérault on mange les jeunes pousses de la clématite en guise d'asperges sous le nom de bidablous. (BARthés, Glous. bot. de Saint-Pons). - En Italie, selon TARgioxi, Di:. bot., la jeune pousse est appelée vitalbino. 
sèche, pour guérir la morve des chevaux, des mulets et des ânes, en forçant ces animaux à l'aspirer par les narines. LoIselectr-Deslongciramps.

Les branehes trainantes servent de cordes à lessive pour faire sécher le linge.

La clématite est employée dans la vannerie grossière, prineipalement pour faire des muselières pour les bœufs et les eheraux.

On en fait des paniers où doivent couver les poules, les pigeons.

Les ehapeliers en font des ronds qu'ils mettent à l'intérieur des easquettes pour les maintenir.

Les enfants, à la eampagne, font désséeher la tige de la clématite et la fument, en guise de cigare.

\section{LOCUTIONS DIVERSES.}

«On appelle vidaoubo une personne dont la taille est longue et effilée. » Corrèze, Bérosie, Dict. du pat. du Bas-Limousin.

« Toumbà dins un beligàs. - S'empêtrer dans une affaire épineuse ou ruineuse. "Aveyron, VAsssier, Dirt. du pat. de l'A $v$.

«Vitalbaio: luogo pien di vitalbe e figuratamente luogo pericoloso. » Italien, Maxuzz, Dizionario ital.

\section{FOLKLORE.}

Le rossignol ehante la nuit parce qu'autrefois il s'endormit sur une branche d'arbre, où il y avait une guidalbe (Clematis vitalba). Les vrilles de eette plante grimpèrent pendant la nuit et lui entortillèrent tellement les pattes qu'il ne put s'envoler; aussi pour éviter d'ètre retenu pendant la nuit, il ehante : dormiraï pu, pu, pu... me toursounaio la vi! (Je ne dormirai plus, plus... m’entortillerait la vigne!) - Périgord. - Poésies pop. de la France, Mss. de la B. Nat., f. fr. 3341, fo 147. Tradition reeueillie par M. DE Gourgues.

(Colta la mattina di S. Giovanni, ha virtù di preservare da' dolori di capo e di ventre chi se ne einge il eapo e i lombi. "Abruzzes, Fisanore.

Pour se guérir du mal de dents on se met sur le pouce une feuille de cette plante. - Lauraguais (Haute-Garonne), c. par M. P. FAgot.

\section{CI.E.MATIS FLA.MILLA. (LINAE). - LA CLËMATITE ODORANTE.}

1. Noms de Cette plante (1):

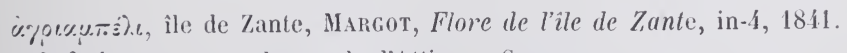

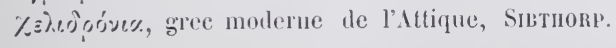

(1) Cette plante porte très souvent les mèmes noms que la précédente, 


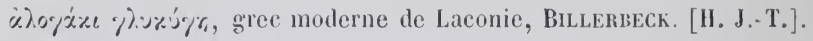
flammula viticelia, clematis urens, anc. nomencl., BAums, 1671. flammula Jovis (1), ane. 110m., Hrac. Anbrosinus, 1666. [H. J. T.]. rincula, anc. nomenclat. savante, J. Canus, Circa instans, n० 198. flammule, f., français du XVIe s., Roch LE BAlluf, Le demosterion, lienues, $1578,1 \% .71$.

clématite odorante, f., clématite flammule. f., français savant.

clamalico, f., sud-ouest du Languedoc, I)erout.

virudelo, f., Castres, A7ass, Dict. des idiomes du midi.

biralelo, f., Saint-Pons (llérault), Bantues. - Sud-onest du Languedoc, DUBouL.

rivonarto, f., Var, Haxrs. - Ile de Porquerolles, Ollivier.

entrevaciis, m., dix-en-P'rovence, Borer de Foxscolonbe.

entrerije, III., Montpellier, Loret. - Nimes, Vixcexs.

jaousémin d'asé (jasmin d'ìne), Apt, Coligxon, - Env. d'Avignon, Palux. jassemi de borro, m., Pyréuées orientalee, Compaxyo, Ilist. n. des P.-O. liseron espagnol, m., français du XVIe s. Lrocien, Ilist. des Pluntes, 1584. vilalba piccola, italien, Targioxi, Diz. bot.

fuoco morto, Vísure et Capri, Pasquale, Flora vesuviana. - Ischia, Gussone.

binsilln, binzigliu, Sardaigne mérid., Spano.

gelsomino bastardo, Emilie, Nomi adoperati a designare le piante di bosco. yeusmi bastard, ritalba odorusa, Bergame et Crémone, Nomi.

brumlinarie rosse, Ldine, Nomi adoperati.

tetli, Sardaigne du Nord, Moris, Fl. surdoa.

ur:ula, Arrizo et Biri (Sardaigne), Ilonis, Flora sariloa.

lisoni, Venise, Boeruo. - padonan, Patriarch.

yüsmina, Brescia, Nomi adoperati.

vidraria, flamula trepadora, espagnol, Colmeiro.

muelmera, Grenade, Borssien, Voyage botanique dans le midl de l'Espagne. vidriella, Minorque, Costa, Flora de Cataluña, 1864.

erbu de flimula, erba de Job, catal., Willkomm et L. [H. J.-T.].

badiella, barlialla, catal., Colmeiro, 1846. H. J.-T.?.

erba de las llagas, catalan, Costa. Fl. de Cat.

scharfe waldrebe, liriechende waldrebe, allemand.

plaméneli, tchèqune.

dirizmina, slirobut, serbo-croate, šctek, Juyosl. im. bilja.

sebenq, arabe algérien, Muxn, Noms arabes des plantes de l'Ali $l_{j}, 1866$. narberd, arabe algérien, HaNoteAt, Ĺa Kabylie.

(1) Aammula dicitur, quod contusa applicat?, veficas flamma instar excitet. Bachis. 
zenzou, arabe algérien, Hanotead. - arabe tunisien, Not. s. les forêts de la Tunisie, 1889.

azen:ou, thouz:imth, kabyle, HaNoteaU.

9. USAGES.

On la cultive aux environs d'Aigues-Nortes, et on en donne les feuilles sèches aux bestiaux qui les mangent avidement, tandis que la plante fraìche est un poison pour eux. - DE LAMARCK et de Candolle.

\section{CLEMATIS IBECTA. (LINXÉ).}

flammula recta, flammula Jovis, anc. nomencl., Bavhin, Pinax, 1671. clematis erecta, nomencl. de DE CANDOLLE. jaoussemi, m., Nîntes, AzaIs, Dict. des id. du midi. entrevalis, un., treverin, m., Apt (Vaucluse), Colignon. entrevige, m., Gard, comm. par M. P. Fesquet. fiammolo, vitalbino, italien, TARgioni, Diz. bot. fiammola, Tessin, Lenticchia. [J. C.] . erba bormera, catalan, Costa. - Vall de Nuria (Catalogne), VaYreda. virumbellas, Vall de Nuria, VAireda. naprasnica, roumain, Branuza, Limba botanica, etc. naprasnic, roumain, Cıнас, Dict. daco-rom. naprasnilie, roumain transylvanien, Fusz, Trivialnamen. blatterzug, Thuringe, Pritzes et Jessen, Vollisnamen. lomons (= casse-ıеz) (1), tomynis, lomynos, petit russien. [Tн. V.] lotol'ina, magyar, Fusz, Trivialnamen..., 1848.

\section{CLEIMATIS VITIGELLA. (LINNÉ).}

clematite bleue, f. clematite i fleurs bleues, français. entrévijhé, m., canton du Vigan (Gard), Rouger. vitalba pavonazza, vitalbino, viticella, italien, TARgions.

\section{CLEMATIS CIRRHOSA. (LINNÉ).}

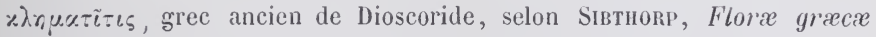
prodomus, 1806.

ùOpurśvn, grec ancien de Théophraste, selon FraAs.

(1) On provoque les saignements de nez en se fourrant des parties de cette plante dans les fosses nasales. 


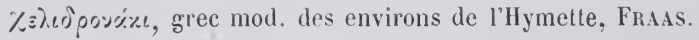
clématite toujour's verte, français, Bon jardinier pour 1843. yerba de foco, anc. espagnol, Ibx Beituar, traluit par Leclerc, II, 430. pidighignu, pidighin:u, Sardaigne du Nord, MoRIS, Fl. sardoa. bintiri:zu, Sardaigne du Sud, Morss, idem. trezzu, Mnravera (Sardaigne), Monıs, ilem. mussorgia, Iglesias (Sardaigne), Moris, ilem. zenะou, ramous, arabe algérien, Muxis, Notice sur les noms arabes. dheyian, arabe, lis Beithar, trad. Leclerc, II. p. 430. zen:ou, gamous, arabe tunisien, Not. sur les forêts de Tunisie, 1889. azen:ou, kabyle, HАхотеAU, La Kabylie. aizen:ou, kabyle, Iвx lieithar, trad. LECL. II, 4:30. thou:zimt, berbère, E. G. PARIs. - arabe du Boghar (Algérie), Dereaux. mara'n, arabe du Ilont-Liban, Berggren, Guide francais arabe, 1844. dâliyyé souda, arabe syrien, BERGGrEx.

dalia souda, arabe algérien, DLRAxDo, Calendrier de Flore alyérienne.

\section{CLEMATIS INTEGRIFOLIA. (LINAÉ).}

klokutze, roumain de Transylvanie, Fusz, Trivialnamen, etc. clocotici, (pl.), roumain, Ciнас, Dict. daco-rom. siberische waldrebe, allemand.

tsiganky (= les bohémiennes), zabiy-kroutcha (= l'ouragan mortel), petit russien, comm. par II. Тн. Volkor.

tsiganka, (plante des Tsiganes), sinii lomonos, Petite Russie, DE GuberNatis, Mythologie des plantes.

bèrtse-rirag, magyar, NEuxich, Cathol. d. Nalurg., 1793.

bértse, magyar, Fusz, Trivialnamen in Siebenb., etc.

\section{FOLKLORE}

En Tartarie, un chef cosatue vaincu, désespéré de voir ses soldats l'abandomner, se suicida avec sa lance. Cet acte de lâcheté ne tarda pas à être crucllement puni, ear il survint hientòt un ouragan qui mit les cosaques en lambeaux et les fit entrer en terre où ils furent transformés en clématites. Saint Pantaléon lés voyant toutes nues en hiver, eut pitié d'elles et leur donna ce duvet qui aujourd'hui couvre leurs fruits. Cependant elles étaient très tristes de demeurer en terre étrangère et ces âmes cosaques finirent par obtenir de Dieu, d'être transportées en Oukraïne pour que les jeunes filles en fissent des couronnes.

Petite Russie, comm. de I. Tн. Volkov. 


\section{CLE.MATIS MAURITIANA. (De Gandolee).}

vigne vierge, f., ile Maurice, BaKER, Flora of Mauritius, 1877.

\section{PULSATILLA VERNALIS. (MILler). - L'ANÉMONE PRINTANIÈRE.}

1. NOMS DE CETtE PLANTE.

anemone vernalis, nomenclature de Linvé.

anémone printanière, $\mathrm{f}$. français savant.

flur de luf, Grisons et Engadine, Durheim, 1856.

anemolo primaticcio, Tessin, Levticchia. [J. C.].

pulsatilla, Vall de Nuria (Catalogne), Vayreds, Catal.

wilde tulpe, allemand, Nемnich, Catholicon, 1793.

waldtulpe, Silésie, Pritzel et Jessen.

schlaapblaum, wilder crocus, Poméranie. Pritz. et Jess.

schafblume, Prusse occidentale, Treichel, Volksth.

eselsylocken. Tyrol, Zwanziger.

ganslan, gugyuhosen, Carinthie, ZwaNziger.

gänserle, Ratisbonne, HopPe, Flora.

oxeöron, gölielilolika, ljælatuppa, lijælsippa, mosippa, movippa, liungsnören, rosli, suédois dialectal, JENSSEN-Tusch.

yjéthivel, norvégien dialectal, IDEM.

verjurt, danois. [H. J.-T.].

On donne souvent a l'anémone printanière les mêmes noms qu'ì l'anémone pulsatille.

9. FOLKLORE.

Die Schlaflume soll nicht ins Haus gebracht werden, wenn die Gänsen brüten, weil sonst die Embryonen im Ei ersticken würden. - Prusse occident., Treichel. Volksth.

\section{PULSATILLA VULGARIS. (MILLER). - L'ANÉMONE PULSATILLE.}

1. NOMS DE CETte PLANTE.

pulsatilla, pulsatilla vulgaris, anemone communis, anemone montana, herba venti, anc. nomencl., Bauhin, Pinax, 1671. 
herba sardoa, nomencl. de DoDoEss, cité par Baunn, idem.

anemone limonia, samolus Plinii, nomencl. de Dalechamps, citée par Baunin, idem.

anemone pulsatilla, nomencl. de LixNé.

anémone, anémone pulsatille, pulsatille, (') français savant.

cocles, f. pl., ane. fr., Duchesne, De stirpibus, 1544; Cir. Etmense, Dict.

latinogallicum, 1561 .

colquelourdes, f. pl. anc. fr., Ducnesne, De stimpibus, $\mathbf{1 5 4 4 .}$

coquelourdes, f. pl. (le mot est presque toujours employé au pluriel),

franç. anc. et mod. - Centre de la France. - Orléanais. - Cham-

pagne. - Normandie.

coqueluriau, m. Yonne, Jossier, 1882.

coquerelles, f. $\mathrm{p}^{\mathrm{l}}$, anc. frans., Brohon, l)e stirp., $15.1 \mathrm{l}$; Duchesne, De

stirp. 1539. - Haute-Marne, comm. par M. A. DAguin.

coqueret, f. Les Riceys (Aube), Guénin, Statist. du cant. des Riceys, 1852.

- Haute-Marne, comm. par M. A. Daguin.

coquerelle, 1., Côte-ll'Or, Roser, Flore, 1883.

clochette, f. fils avant le père, m., herbe à la teinture, f. Hante-Marne, comm. par M. A. DAguin.

couchot, m., Lemmecourt (Vosges), Haillant, flore pop. des Vosges. couchiri, m., Meuse, Cordier, Voc. des mots patois de la Meuse, 1833.

couchiri, m., coucheriu, m., coucher'u. m., couchereu, m., coucheriol,

III., Meuse, Labourasse, Gloss. du pat. de la Meuse, 1887.

coucherieu, m., Mense, Doisr, Ess. s. l'hist. nat. de la Meuse; Labourasse.

Giloss. du put. de la Meuse.

coucou, m., Centre de la France, Jaubert, Gloss. du C'entre. - Côte-d'Or,

Roser, Flore de la Côte-d'Or, 1883.

canquoin, m. Chàtillon-sur-Seine (Côte-d'0r), c. par M. F. Dagurs.

canquoins, pl., cougjus, pl., Côte-d’or, Rover, Idem.

coincoin, m., Haute-Marne, comm. par li. L. Aubriot.

fleur de Pâques f., Haute-Narne. - Normandie.

flou de Pasio, f. Forcalquier (B.-Alyes), c. par M. E. PLacchub.

passobélours, erbo d'entée (²), Lot-ct-Gar., e. par M. l'abbé L. Dandr.

passetelours, m., passefleur, français du Xiı s., DuEz, Dict. françois-

ital. 1678.

erbo del vent. f., Gard, comm. par M. P. FesQuet.

(1) Le mot pulsatille est déjá dias Cotgrave, a french. dict., 16 iso.

(2) Entée signifie poison lent, langueur. - Les paysans atteints de fière intermittente lappliquent autour du poignet, à l'endroit des pulsations [.luhé L. DARDy]. Cumparez le nom die la plante: pulsatille. [E. R.]. 
herbe au vent, f., Champagne. - Normandie. - Bourbonnais.

écorche viau, Aube, Des Etangs, Noms pop. des pl. de l'Aube, 184.4.

herbe au liable, f., Aube, Des Etangs, Idem. - Canton des Riceys (Aube)

Guénin. Statist. du Cant. des Ric. 1853.

flour de lou, f., Briançonnais, comm. par M. J. A. Chabrand.

tête de loyze, f. Suisse romande, Vicat, Hist. des plantes vénéneuses.

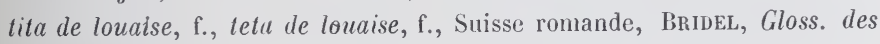

pat. de la Suisse: Durhem, Schweiz. Pflanzen-Idiotilion.

gironato, f., Var, Avic, Catal. des Plantes du Var.

mourgue, f., Cantal, recueilli personnellement.

coripiène, culotte, Normandie, CHesnow, cité par JoRET.

passafior, fior de Pasqua, erba de vent, milanais, Cherubin.

erba del diavolo (1), italien, Duez, Diz. ital. franz., 1678.

stelaro, Venise, BoErio, Diz. del dial. vene:.

flor del vento, espagnol.

erba do vento, gallicien, CuveIro, Dicc. yallego.

adormitzele $\left({ }^{2}\right)$, f. pl., deditzei $\left({ }^{3}\right)$, pl., oitza, flórea-vîntului, flóreapascelui, roumain, HASDEU.

tagschlaf, tagschlag, ancien allemand, JIEFENBACH, Gloss. lat. germ. merl. aet., 1857 ; s. vº pulsatilla.

bergmündel, bergmünnlein, allemand, Grimм.

kuhschelle, küchenschelle $\left(^{4}\right)$, osterschelle, osterblume, bocksbart, mutterblume, wildmannshraut, tageschlaf, das graue bergmännchen (wegen des grauharigen Kopfes den man nach der Blüthe auf dieser Pflanse sieht), allemand, Nемnich, Catholicon, 1793.

krônblôm, bas allemand, Grini, Wörterb.

hackelkraut, Prusse, Frischbier, Preuss. Wörterb.

beisswurz, bisswurz, Suisse, Nemich, Catholicon.

arschkucke, Autriche allemande, Nвмліси.

arstguclin, aschgub'n, blaue arschlium, merznbêcherl, oarguka, BasseAutriche, F. HoEFER.

schlotterblume, cantons de Schwitz et d'Unterwald, Durheis, Schweiz. Pfl. Idiot. - Prusse, Frischbier.

(1) Ainsi appelée parce qu'elle fait du tort aux blés. (DuEz.)

(2) C.-ì-d. les petites endormies. - Le calice de la fleur de la pulsatille se referme, quand vient la nuit.

(3) Ciнsc, Dict. daco-roman, explique ainsi ce mot : de dadacesc = faire la bonne d'enfant, soigner un petit enfant. On emploie la plante tout entière pour les bains des petits enfants.

(4) Par fausse étymologie populaire. 
heuschlafen, f., Souabe, Scumid.

güggelblume, Suisse allem., Pritzes et Jessen, I). d. Volksn. d. Pfl.

ruug moderkrut, IIolstein, Idem.

tagschläferle, Henncberg, ReinwaLD, 1793. H. J.-T.

wolfspfole, bords de la Moselle, Pritzel.

plumpblomen, allem. tranșylvanien, Pritzes.

uihstereblomm, biereblommen, allem. de Transylvanie, FLsz, Trivialnamen in Siebenb.

kloclienblome, environs de Brême, Focke, Volksth. P/lunzeniamen, Brême, 1870 .

mânskraut, Luxembourg, J. WEBER.

oschterblum, Luxembrurg, KoLT\%, Flore du Grand Duche, 1873.

kiuchenschell, Alsace, MAPPus, Historia plantarum alsaticarum, 1742.

bilzuu':, allemand, Diefenbaci et Wuelcken, IIoch und niederdeutsches

Hrerterb.

belswort, moyen bas allem., Idem.

beizulü, Saxe, liaumgarten, 1790. H. J.-T.]

kiueclienschelle, flamand, DodoxaEs, 1644. A. DE C.]

wildemansliruid, flamand, Otdemaxs, 1872. A. DE C.]

pasque flow'er, anglais.

passe flower, flau flower, anglais du XVIJ siècle, CoTgRave, French dict., 1650.

dane's blood, dane's flower (1), Coventry bells, anglais dialectal., BRITTEN et Holland.

blodeuyn y pasc (= fleur de Pìques), gallois, Hugh Davies. [H. G.]

coculoce, breton, Nomenclator de 1633. [E. E.]

coculoc: , breton, P. GrégoIre. E. E.

oxöron, ysnöron, lioskälla, ïxnabjülla, vippa, bakktulpan. bakksippa,

tiälroser, suédois dialectal, Jexssen-Tusch, Nordislie plantenavne, 1867.

kubjelle, norvégien, Idem.

bli̊ vejrurt, bli̊ vorurt, bli liobjelde, blï oxegre, danois dialectal, Idem. pohanina, pulzlatnili, tchèque, A. Müller, Wört d. off. Pflanzen, 1866. sasa, poklonac, kosmatinec, serbo-croate, Šulek, Jugosl. im. bilja. sasantia, polonais.

sanki, sek, polonais, Er.ntel, Warsavia physice illustrata, 1730. czarne ziele, polonais, LINDE.

(1) Cies noms sont une corruption par fausse étymologie populaire des mots Adonis blood, Adonis flower. 
son-trawa, wetrezina, prostrel, russe, Falk, Beytr. z. topog. Kenntn. des r'uss. Reichs, 1786.

nahas-loraki, basque, LACorzQLETA. [J. V.].

tavaszi, kokoertsin, magyar, Fusz, Trivialnamen.

karro liepparl, esthonien, HuPEL.

hawaye, arabe syrien, BerggRex, Guide français-arabe, 1844.

sortschup, kirghi\%, Falk, Beytr., 1786.

«L'ensemble des fruits plumeux de l'anémone pulsatille est nommé cla boussel; au figuré on appelle chaboussel un homme qui a les cheveux hérissés et mal peignés. „) Briançonnais, comm. par M. J.-A. CHAbRAxd.

Les fleurs de celte plante servent à teindre les œufs au temps de Pâques.

\section{PULSATILLA PRATENSIS. (MILLER).}

anemone pratensis, nomencl. de Linné.

pulsatilla nigricans, nomencl, des pharmaciens, XVIII siècle.

barllanto, f., provene. mod., AzAss. Dict. des idliomes du midi.

sisinei, roumain, Brandza, limba botanica, 1882.

wiesenliuhschelie, allemand.

osterblume, Prusse, Frischiler, Preuss. Würterb. 1882.

bitzwurzel, allemand, Grum, Wörterb.

glockrose, Brandebourg, Pritzel et Jessex.

schwarze arschtgucken, heanaug'n, heanglock'n, schafgloclien. Basse-

Autriche, F. HowFER, Woerterb.

sasanlia, pohanina, tchèque, A. Mueller, Alph. Wörterb., 1866.

ozarne ziele (= herbe noire). polonais, NEмnich, Cath.

lesjali mali, mali lesjak. sasa, zaspanka, serbo-croate, ড̀Ltek.

son blakitny (= sommeil bleu), prostril (= traversant comme une flèche),

petit russien, comm. par М. Тн. VоцкоV.

leany liukörstin (= herbe noire), magyar, Nemsich, ('atholicon, 1793.

numanu perri, turc, Plıarmacopea româna, 1862.

\section{PUISATILLA MONTANA. (MILLER).}

anemone montana, nomencl. de Lixsé.

anemouno de mountayno, f., Forcalquier, c. par M. E. Platchid.

graulon, Forez, A. Legrand, Statist. lotan. du Forez, 1873.

osterglöckchen, Tyrol, P'ritzen et Jess., D. d. Volksn. d. Pfl.

\section{PLLSATILLA I'ATENS. (MILLER).}

anemone patens, ncmenclat. de LiNsÉ.

heideblume, Prusse, Fisischbier, Preuss. Wörterb. 
arschglocken, erstyucter, Basse-Autriche, F. HowfER. wetrenik, wetreniza, russe, FaLk, Beylr., 1786.

son (sommeil), son trava lierbe du sommeil), son blakitny (sommeil bleu), petit russien, comm. par M. Тн. Volkov.

On dit en Russic que si l'on place cette plante sous l'oreiller, elle vous fait voir en rève ce qui vous arrivera en réalité. - DE fubernatss, Mythol. des $p l$.

\section{I'LLS.ITILL.1 ILI'IAI. - (LOISELEUR DESLONGHAMPS).}

anemone alpina, nomenclature de Lixxé.

éponsè, f., =épouse), canton d'Aime (Savoie), comm. par M. Marjollét. amimone, f., Montreux (canton de Vaud), Durheim.

pel de rat, m., sud-ouest du Languedoc, Duвoul.

péou d'arrat, m., (= poil de rat), Luchon (Pyrénées), J. SACAze.

pei di $c u . m$, Alpes vaudoises, Durheim.

barbua, f., provençal mod., Hoxvorat, loc. provenc.

barbudlo, f., Gard, comm. pa. II P. Fesquet.

booumiano, f., Forealquier, c. par M. E. PLALChUd.

viola blanca, catalan, Costa,, Intr. à la Flora de Cat. 1864.

pulsatilla, Vall de Nuria (Catal.), Vayreda, Catal.

bürentatzen, wilder jager, schnecrosen, räucliliny, weisser rüuchling, rïucherlan, Carinthie, Zwa৯zIGER.

haarige mann, Berne, Dunheiм, Schweiz. Pflanzen-Idiot.

haarmanteli, Oberland bernois, IDEM.

teufelsbart, Silésie, Pritzel et Jessen.

sidahuat, schaudermann, fotzabäsa, tifelsbart, bergmünnli, canton de Saint-

(iall, Wartmaxx, Beitr. :. Saint-Gall. Vollisbot., 1874.

hoar im at'st, schnearoase, Basse-Autriche, F. HoEfER, Wïrterb. 1. niederïsterr. Pflanzenn. - Carinthie, LExER, Kärntisches Würterb. 1862.

\section{ANEMONE NE'MOROSA. (LINEE). - L'ANÉMONE DES BOIS. 1. NONS DE CETTE PLANTE :}

anemone sylvestris vulgaris, ranunculus candidus, ranunculus nemorosus, ranunculus sylvarum, anc. nomencl., Baunin, Pinax theatri botanici, 1671.

anémone des bois, f., renoncule des bois, f., anémone sylvie, f., sylvie, f., sylvie blanche, f., français.

anemona dels prats, f., Pyrénées-0rientales, Companyo, Hist. nat.

alimoïno dei boués, f., provençal, Lrons, Ess. s. les végét. des Bouchesdu-Rliône, 1863. 
anemouno du bouos. f., Forcalquier, c. par II. E. PLauchud.

limouna, f., provençal mol., Honnorat, Voc. provencal, 1848.

alèmânne, f., Saint-Pol (Pas-de-Cal.), c. par H. Ed. Edıont.

amimone, f., Montreux (canton de Vaud), Durhein.

bassin blanc, m., bassinet blanc, m., Haute-Marne, comm. par M. A. DAGUIN,

risoletta, f., Château d'OEx Suisse romande), Vicat, Pl. vénénenses. Bridel, Gloss. du pat. de la S. rom.

tourne-midi, casse-verre, Haute-Marıe, comm. par II. L. Iubiot.

queullon-pä̈ele (= chandron-poële), Saint-Just (Oise), L'Intermédiaire, Vol. I, p. 169.

coucou, m., Normandie, JoRet, fil. pop.

flenr anx cocus, f., Centre de la France, Jaubert, Gloss. du Centre.

passe-fleur, pihatte é lé, f., Spa, LezAAck, Jict. des noms n'allons.

sochelî, Plancher-les-llines (Haute-Saône), Poulet

solhlüye, f., Vallée de Cleurie Vosges', Thiniat, La vallée de Cleurie. sohlâye, f., Vosges, Haillant, Flore pop. des Vosges.

hhohhlauye, f., Cornimont (Vosges), IdE.M.

hhohhlane, f., fonrfelane, f., La Bresse (Vosges), Idem.

fourfelae, f., Gerbamont (Vosges), Iden.

fourfelaye, f., Vagney (Vosges), IDEM.

jeannette, f., Bainville (Vosges), IDE.M.

Ilemoiselle, f., Normaudı, JoRk, fil. pop.

fleur des dames, f., Normandie, Ion:.

bilés de chenve, Ban de la Roche, H. G. Oberun, Description géognostique. écorche viau ( $\left.{ }^{1}\right)$, Aube, Des Étaxgs, Noms pop. des pl. de l'Lube, 1841.

pormmerole. f., cloche, clochette, claqnets, m. pl., Normandie, Jonet.

pâquette, f., Oise, Graves. - Herlin-le-Ser (Pas-de-Calais), c. par M. Ed.

EDsont.

fleur de Pâques, f., normand, JoRET.

fleur du vendredi saint, f., Oise, (iraves.

fleûr do vinrli sin, f., fleur du printin. f.. flevir du mä (lleur de mars),

yejp' du moss (herbe de mars), blan mâ, pihèlé, m., hit' l'oủhê, wallon. FELLER.

rosella borla, Vall de Nuria (Catalogne), Varrebs, Catal. de la Flora. mapóla silvestre, gallicien, VALLADARES.

oit:a, flórea vintulni, flórea pascilor, roumain, BRANDzA, Limba botanica. floare aschtilor, mustenitsch alb, roumain de Transylvanie, Fusz.

(1) Cette plante cause le pissement de sang et la dyssenterie aux vaches. 
weisse osterblume, müræblïmchell, windrïschen, kiiseblume, kï̈seblüınchen, weisse winlblume, buschwindrïschen, allemand.

katzenblume, kï̈blume, Henneberg, Pritzel et JEsSEx.

luk, Tubingue, IDEs.

moryendiimincher, allem. de Transylvanie, IDE.I.

schneekaterl, Salzbourg, IDE.u.

segenblanme, Gottingue, lDE.s.

abrelblum, beschblum, liesblimchen, lieschtblimcheu, hol:blum, leffelliraut,

hefflerblum, Luxembourg, J. Weber.

waldhiincheu, Oldenbourg, Focke, Volksth. Pflanzenu.

storchenblume, Suisse allem., Duruerm, Schu'-Itliot.

bettseicha'a, osterbluoma, eierbluoma, willi sitlosa, tubateckel, zitlosa,

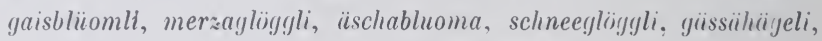
gaissanägeli, eanton de Saint-Gall, W.ntmans, Beitr.

yänsbliimll, canton des Grisons, Derhein, Schw.-Idiot.

gäsglöggli, cant. d'Appenzell, IDEx.

kronhaxn, Basse-Iutriche, F. HöFE, Wört. Il. niedisterr. Pflan:enn. naaken hiemallien, naalite wiewlien, Westphalic, LAxdors, Die westfüli-

schen Pflatzemanen (dans Botanisches Centralblatt, 1882.

kïisbliimichen, liuchulisblume, Eifel, Wirtgex, Vegetation der Eifel, 1865. milhlradeln, Garinthie, Zwaxziger, Verzeichn.

bosch-hanen.voet, flamand, DoDoxatess, 1641. [A. de C.]

mellibloem, bosch-hanevoet, windbloem, flam., Ocdexass. [1. de C.]

kwade-oogen-bloemen, ber'stbloemen, eierkens, Flandre orientale, c. par

II. A. DE Cock.

melli-nortel, Groningue, DE GoRTER.

alremony, emony, woorl crowfoot, anglais, PRior, Pop. names of $\mathrm{br} \cdot \mathrm{pl}$. enemy, anenemy (par suite de fausse etymologie populaire', anglais dia-

lectal, Brittex et Hollaxd, Dict. of engl. planl-names, 1878.

cuclioo flower, granny's nightcap, anglais dialectal, IDE..

couslip, nord de l'Écosse, IDEM.

bowbells, cuclioo spit, Devonshire, Friexd, Gloss. of Devoush. plant-names,

1882. - West Worcestershire, Cinnberlaix, Gloss. of Worcestersh. lus ua gaoithe (= plante dı vent), irlandais, FoLEx. [H. G.]

plir ua gaoithe (= plante du vent), gaèlique écosiais, C.AMERox. [H. r.] lus na geajee (= plante du rent), manuois, Cregees et lielly. H. G.] bloclau' $r$ gu'ynt (= fleur dı rent), ffrithogen (= forestière), gallois,

Yeddy!gon Myddfai. [H. G.]

blodeuyn y gwyut (= fleur du vent), llys y grwgut (= plante du vent),

ffrithlys (= plante du taillis), gallois, HuGH Davies. H. G.]

nead chailleach, [ = old woman's nest , irlandais, 0 Reills. H. G. 
disgral, breton, D. Le. Pelletier. [E. E.]

dislirab, breton, LiéGARD. [E. E.]

hvitsippa, hvithverf, hvitmeja, tjälblomma, luki, suédois dialectal, Jenssen-

Tusch, Nordiske plantenaine, 1867.

simmer, kvilvis, hvitveis, hvidsimmer, norvégien dialectal, loEM.

hvidvisse, hidsippe, snogeblombster, danois dialectal, IDEM.

rüsiclia hajni, tchèque, A. Ntelder, Alph. Woerterb. 1866.

ranilica, breberina, rupelnica, minica, serbo-croate, šulek,

louroslip (= cécité des poules) (1), petit russien. comm. par М. Тн.

VolKov.

fejer bereli-viray, magyar. Fusz, Trivialnamen in Siebenb.

fejér pipats, magyar, NeMnjch, Catholicon, 1793.

fedjela, arabe algérien, Florian Pharaon, Voc. ar., 1860.

\section{FOLKLORE.}

Die linder pflegen die drei ersten Windröschen, welche sie im Frühjahre funden, zu essen, weil sie danı das ganze Jahr hindurch niclıt krank werden. ") Prusse occidentale, Treichel, Voltksth. aus der Pflanzemelt $f$.

Westpreussen.

Cf. "Magi multum quidem iis tribuere, quumprimum aspiciatur, eo anno tolli jubentes; dicique, colligi eam tertianis et quartanis remedio. Postea alligari florem panno roseo el in umbra asservari ita, quum opus sil, adalligari." - Les mages ont attribué de grandes vertus à ces plantes (aux anémones), ordomnant de cueillir aussitôt la première qu'on aura aperçue de l'aunée, et de dire qu'on la cueille pour guérir de la fievre tierce ou de la fiève quarte; après quoi on enveloppera la lleur dans du drap incarnat, on la gardera à l'ombre, pour la porter en amulette, quana il en sera besoin. PLine, Hist. nat. édit. Littré, XXI, 94.

ANEMONE CORONARIA. LINNÉ). ET ANEMONE HORTENSIS. (LINNE) (2). - L'ANEMONE DES JARDINS. xous.

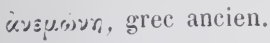

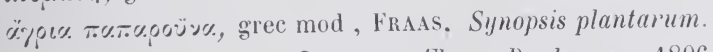

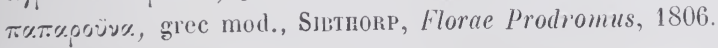

(1) On appelle ainsi certaine maladie des yeux. Celui qui en est atteint ne voit plus clair à partir du coucher du soleil, mème auprès d'une lampe allumée. [Th. V.]

(2) Cette dernière est l'anemone stellata de LaMarck. 


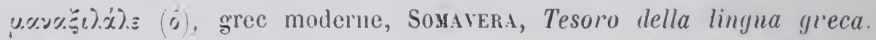
anemone, latin.

anemone sativa, ancienne nomenclature.

anémone, f., anémone des jardins, f., anémone des flenristes, f., anemone cultivee, f., frangais.

anemouno dei jardin, f., anemouno des ouort, f., Forcalquier, comm. par M. E. Platchud.

animone. f.. Valenciennes, HЁсавт.

couculo, f., anemouno, f., sud-ouest du Languedoc, Deisout.

iol de perdigal, m., Ağde (Hérault), Azass, Dict. des idiomes rom.

anemone, anemone de yiardino, anemolo, italien.

anemola, napolitain, Savastaxus, Botanicorum libri IV, Neapoli, 1712, p. 93.

anemul. Frioul, Pirona, loc. friulano. - Bologine, Coronedi-Berti, Voc. nèmula, fran:esiglia, sarde méridional, SPAxo.

anébolo, m., Gènes, Casaccia, Dict. genov.

anemolo, anemone, $\mathrm{m}$. sarde logodonrien et septentrional, Spaxo.

anemone areste, Sardaigne, Monis, Flora sardoa.

fayottino, lindadóro, italien, TARgioni, Diz. bot.

paparinedda az:ola (') (?), Sicile, LAGLsı, Erbuario-italiano-siciliuno,

Napoli, 1742, in-4.

aryemone, Sicile, CuPAxi, Hortus catholicus, 1696.

argemolo, aryemolo dal fioco, Vérone, PoLbixi, Flora veronensis.

anemone de coronas, anemone coronada, espagnol, Nerxich, Cath.

nemora, catalan, Colmeiro, 1846. [H. J.-T.]

anemone, anemola, portug., Brotero, Flora lusitanica, 1804.

anemona, gallicien, VALLADARES.

kronenanemone, gartenanerione, anemone, allem.

salamönli, sulomönli, Suisse allem., Durhern, Schweiz. Illiot.

alemönli, Suisse allem.. Pritzel et Jess., D. d. Vollisn. d. Pfl.

tuin-anemone, hollandais, Neмxich, Catholicon, 1793.

gallant, ancien anglais, Brittex et HoLl., Dict. of. engl. plant-names.

trägairds-sippa, sucidois, Хемхıсн, Catholicon. 1793.

kalos, serbo-croate, šclek, Jugosl. im. bilja.

an-no'mân ('), cheqầiq an-no'man, arabe.

(1) L. gousi traluit paparinedda a:sola par anémone sauvage. De quelle anémone verutil parler?

(2) Selun Dozr, Gloss. des mots déricés de l'arabe, p. 373, et DE SLANE, Traduct. 
na'moun, arghamoûni, arabe syrien, BERGGRE, Guide français arabe. lala, arabe algérien, Munbr, Noms arabes des pl. de l'Algérie, 1866. kahwiela, Malte, Delicata, Flora melitensis, 1853.

On trouvera les noms français des rariétés d'anémones cultivées, dans VALLOT, Hortus regius, 1665, p. 13-19.

Les jardiniers et les fleuristes se servent, tans la culture de ces fleurs, de termes particulier's qu'on pourrait appeler laargot des amateurs d'anémones. Voir ces termes dans Rozier, Cours d'agriculture, 1793, sub verbo anémone, p. 477-483 et le Bon jardinier pour 1811, 1. 141-149.

\section{FOLKLORE.}

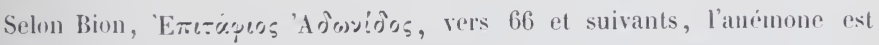
née des pleurs de Vénus, et la rose du sang d'Adonis. Selon Nicander, daus ses scholies sur Théocrite, V, 92, c'est l'anémone qui est née du sang d'Adonis.

\section{ANEMONE P.A TONTA. DE GiNdolde) - ANEIIONE OEIL DE PAON.}

anemone pavonina, nomencl. de LAMARck. griffe de chat, f., Centre de la Frauce, Jacbert, Gloss. du $C$. weil de paon, m. français. iol de parou, m., Agde Hérault). Azass, Dict. des idiomes du midi. olho de parào, portugais, Brotero, Flora lusitanica, 1804.

\section{A.VEMONE HEPATICA. LIXE' - LHÉPATIQUE ${ }^{1}$.}

trifolinm hepaticum, hepatrcum trifolium, trifolium magnum, trifolium aureum, hepatica. hepotsca trifolia, hepatica terrestris, herba trini-

angl. d'Ibn Khallicin, II, p. 5T, le mot arabe an-no'man est une corruption du grec ¿̀su(s)r. - Les Syriens expliquent ce noin, en disant que No'man, le célèbre roi arabe de Hira, ayant le premier introduit cette plante dans son pays, lui donna son nom. M. Clermont-Gaxieau (Etudes d'Archéologie orientale, Paris, 1880 , p. 26 et suiv.) pense que le No'man dont il est question, n'est pas le roi de Hira, mais une divinité de l'ancienne Syrie, correspondant à Adonis.

(1) Ciest une plante dont les racines fibreuses poussent des touffes de feuilles à long pétiole, nombreuses, d'un vert luisant, tavelées de blanchâtre, divisées en trois lobes ; ce qui leur a fait trouver quelque ressemblance avec le foie, et a valu à la plante le noun

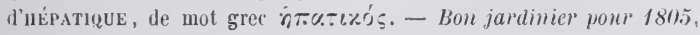


tatis, trinitas, nomenclature sarante dn Moyen-dge et do la Renaissance, Baunis, Pinax llieatri botanici, 1671.

trinitas-unitas, latin du XVe siècle, G. Canus, L'opera salernitana. hepatica triloba, nomenelat. de DE CANDOLle.

hepatica nobilis, nomenclat. de МоExch.

liépatique, f., hépatique des jardins, f., anémone liépatique, f., anémone

bleue, f., herbe de la Trinile, f., français.

liepatique des fleuristes, f., hépalique lrinitaire, f., français, Banbeu-

Dersocro, Le Botaniste fransois, 1767. [Ed. EDs.].

palica, Valenciennes, Hėcart. (terme corrompu introduit par les jardiniers.) erlba del fetje, f., erba del bajou, f., buxol, Pyrénées-Orientales,

Companio, Hist. nat. des Pyr.-Orient.

erba d’uou féyé, f., Gard, PouzoLz, Flore du Giard.

erba d'aou fétylié, f., Le Vigan (Gard), RocGer, Topoyraphie.

crbo d'oou fégé, f., Aix en Pror., Borer de Foxscolombe. - Var, Haxrs.

- Apt (Vancluse), Colignox.

erbo dou fège, f. Forealquier, c. par M. E. Pladchud.

erba au ferlzo, f., vandois, Derhem, Sclur. pfl. idiol.

eirl)' au fedje, f., ourellella, f., Suisse romande, BRIdes, Gloss. des pat.

lierre de terre, m., Beaumesnil (Normandie), JoRkt.

rioleto dé Sanle-Madeleine, f., Aix an Provence, Borer de Foxscolonbe. anemone feyatella. italien, Nesnich, Catholicon.

erba figadela, Venise, Boerso, biz. del dial. renet.

figarlela, Brescia, Melchori, Voc. bresc.

erba Trinita, erba Trinitas, Trinitas, rammeolo tridentato, trifolio

epaliro, italien, TARGioni, Dis. botaniro.

piadasna, f., romagnol, MorRt.

erba felyera: catalan, Costa.

erba da Trinilade, gallicien, Cureino, Dicc. gall.

\& yerba de la Trinitarl, esp)., Colveiro; aragonais, Willkom. [H. J.-T.]

trebol de prados, isp., Cussius, 1601. [H. J.-T.]

higadela, espagnol, Ouds, Tres. des deux langues esp. el frans., 1660.

crucea roiniculmi (= la croix du fort), trei-rei (= les trois méchants),

roumain, Braxdza, Limba bolanica, 1882.

foi de juare, roumain de Transylvanie, Frsz, Trivialnamen d. Pfl. leberblume, leberkraul, hersliraut, blane osterblume, edel-leberliraut, allemand.

gulden kilee, allemand, Cussius, Rar. plant. historia, 1583.

grillenlilee, allem., Loxicerus, 1540; Dodoexs, 1644.

fisawan lepèr, m., Luxembourg, J. WEBER.

blatterliraut, allemand, Grum, Deulches Wort. 
nomutzchen, Paderborn, Pritzel et Jess. Vollesn. d. Pfl.

haselmü̈nch, Tyrol, I. V. ZINGerle, Wali, Büıme, ete. (dans Zeitsch. I. 1. d. Myth. I, 323.)

katoenaug'n, lebalirand, Autriche allemande, F. HoEfer, Wört.

mür\%blume, Prusse, Frischbier, Preuss. Worl.

labarablïamli, steibliiomli, merzablüomli, Saint-Gall, Wartmann.

mühliblüamll, Sargans (Canton de Saint-Gall), Wartuans.

blaue violen, Prusse orientale, Pritzel et Jessen.

blaue holzblume, Henneberg, Pritzel et Jessen.

liewerkrockt, hasselvoaltcher, allemand de Transylvanie, Fusz, Trivialn.

apateka (1), llamand. [A. de Cock].

liver wort, anglais.

Trinity, herls Trinity, noble agrimony, noble liverwort, three leaved

liverwort, anglais, Cotgrave, French diction., 1650.

squirrel cups, heart lirerworl, spring beauly, Etats-Unis, BERGEN.

liframert, islandais, Jenssen-Tusch, Nordiske plantenaine.

lefverört. bleisippa, lïkk, blülälik, lillingblomma, suédois, IDE.M.

blüsimmer, blüreis, norvégien, IDE.

leverurt, adelklever, blïsimmer, y!glenlilever, danois. H. J.-T.]

jalermick, podlislia, tchèque, 1. Míller, Wört. Al. off. pfl., 1848.

alencik, jatrenka, trojica, maćlia, tetenek, serbo-croate, ŠuLEk, Juyost. trilistnik (= trois feuilles), russe, FaLk, Beytr.z. Kenntn. d. russ. R.

liviat watrobiamy, polonais de la Prusse, Treichel.

matrobnili, polonais, EnNotel, Warsar. phys. illust.

alinu sahle, letton, Uluaxn, Lettisches Wïrt.

pehlera sahles, alimabding, letton, HupeL.

liülma-alıne, lï̈lma-elıjal, lï̈lma-elased, esthonien, Wiedemans.

maksa rohhul, esthonien, Hupel.

mäjfï, magyar, Fusz, Trivialn. d. Pfl.

Remarque importante. - Ve pas confondre cette hépatique qui appartient à la famille des Renonculacées avec la pulmonaire, espèce de lichen qu'on appelle quelquefois aussi hépatique.

\section{ANEMONE RANUNGILOIDES. (LINNÉ). - LANÉIONE A FLEURS JAUNES.}

ranunculus nemorosus luteus, anemone nemorum lutea, ancienne nomenclature, Bauns, Pinax theatri botanici, 1671.

anémone à fleurs jaures, f., sylvie jaune, f., fausse renoncule, français.

(1) Par corruption du terme savant hepatica. 
elléborine, f., français des jardiniers, Le Bon Jardinier pour 1805. casse-verre jaune, Haute-Marne, comm. par M. L. Auвriot. yelbe osterblume, allemand. aprilblume, märablume, Prusse, Frischiser, Preuss. Wört. yoldhülınchen, geelögschen, gelbe waldveilchen, Silésie, P'RITzEL ct JEss. gelbe wallviolen, geele haselblumen, Prusse orientale, IDEN.

gul simmer, danois. [H. J.-T.]

yulsippa, suédois, Jennsen-Tuscu, Nord. planten.

guldsimmer, gulveis, norvégien, IDEn.

śuta śumarica, polc̈nili, časa, serbo-croate, ŠLlek.

kozelet:, petit-russien, comn. par M. Th. VoLkov.

\section{AIOONIS ESTITALIS. (LINÉ). ET MI)ONIS \\ ALTIMNILIS. (1) (LINNÉ). - LES GUUTTES DE SANG.}

up\%zucisy, gree ancien de Dioscoride, selon Blllerbeck, 1824, et FraAs.

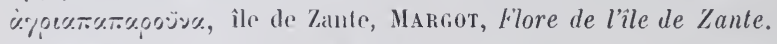

adonium (") (??), latin, PliNe, Ilist. nat.

adonis, flos adonis, adonium, anemone tenuifolia, anemone ayrestis, eranthemum, chamsmelum eranthemum vel purpureum vel rubrum, aculeata, ranunculus arvensis flore rubicundo, anthemis eranthemos, Nomenclat. du Moyen-Age et de la Renaissance, BavHin, Pinax theatri botanici, 1671.

helleborus Hippocratis $\left({ }^{3}\right)$, nomenclature pharmaceutique ancienne, Rosenthal, Synopsis plantarum diaphoricarum, 1861.

goutte de sang. f., gouttes le sang, f. pl, sang de Vénus, m., sang de Jésus, m., français.

(1) Ces deux plantes sont quelquefois confondues sous te nom de Adonis annua.

(2) I propos de ce mot Eug. Founxier a inséré dans le Dict. botanique de Ballon, 1876, t. 1, p. 53 , le petit art. suivant:

Idonium, plante inventée par Pỉne, qui a mal rendu, comme cela lui est arrivé souvent, le texte de Thejopluraste et qui a traduit par adonium les mots 'A ơvídos jardins $\mathrm{l}$ Adonis.

La mème opinion avait déjà ité émise par LitTré dans sa traduction de l'Histoire naturelle de Plixe, t. II, I. 73, note 10.

(3) Clusius rapporte que de son temps ( $\mathrm{XVI}^{\mathrm{e}}$ s.) les pharmaciens allemands substituaient sil racine à celle de l'hellebore et la regardaient mème comme le véritalle hellébore d'Hippocrate a cause dune sorte de ressemblance extérieure avec la raciuf que le père de la mèlecine décrit sous ce nom. 
goutte ed' sang, f., Saint-Pol (Pas-de-Cal.), c. par M. Ed. Edmont. gottes di sonk, f. pI. wallon, FELLER.

fleur d'Adonis, f., Duez, Diz. ital. franz. 1678.

saigne-nez, Anjou, Desvaux, Flore de l'Anjou.

coq-thé, m. Loiret, c. par M. J. Poquet.

bassin. Jouy (Loiret), c. par M. J. PoQuet.

rubitz, m. anc. francais, DECAISNE, Catalogue des plantes représ.

roubisso, f. environs d'Avignon, PALun, Cat. des plantes d'Avignon. Gard, comm. par M. P. Fesquet.

rubisso, f., $\Lambda_{\mathrm{p}}$ (Vaucluse), Coligion, Flore d'Apt. - Bouches-du-Rhòne,

Villeneuve, Statisl. des B.-du-Rh. - langmedocien, Sauvages, Dict. rougeotte, f., Mínil en X. (Vosgess), Haillant, Fl.pop. des l'osges. Haute-Marne, comm. par M. A. Daguin.

brunette, f., Valenciemnes, HéCart, Dict. rouchi.-Sornme, Ledieu. - Hante-

Marne, comm. par M. A. Daguin. - Moyemmoutier (Vosges), Halllant. roouro bastardo, f., gouto de sang, f., flou dou masclun, f., Forcalquier

(Basses-Alpes), e. par M. E. Plauchud.

rouelo bastardo, f. (unot à unot faux coquelicot), Apt (Vaucluse), Colignon. seil de perdrix, m., Haute-Marne, comm. par M. A. DAguin. - Normandie,

JoREt, $F l$. pop.

ail de faisan, m. français, Vilmonin, Fleurs de pleine terre, 1870.

ail du diable, m. français, Dict. d'hist. nat., 1804-1830. [H. J.-T.]

galan, (') m., Toulouse, Tournos, Flore de Toul. - Tarn-et-Garome,

Lagrèze-Fossat. - gascon, Noulet, Flore.

erbo d'amour, f., Var, Haxkx, Catal. des pl. du Var.

aourignol, il. sud-onest du Languedoc, A. Duвoul.

moussurèt, m., (= petit monsieur), aouriflan routje, m. sud-ouest du

Languedoc, Duboul. - Tarn-et-Gar., Lagrèze-Fossat.

boulech rouge, m. Villefranche de Lauragais (Haute-(iar.), c. par M. P.

FAG0T.

gratê, masculin pluriel, Charente-luférieure, comm. par M. E. LEMarié. jalbert salbage, m., Hérault, Bartiès, Gloss. bot. de Saint Pons.

bramèfouam roudze, Issoire (Puy-de-Dôme), comm. par M. J. Bareire.

fior d'Adone, ranunculo dei grani, italien, TARgioni, Diz. bot.

adonide, occhio di diavolo, italien.

adonio, ital., Duez, Diz. ital. franz., 1678.

gozze de sangue, Vérone, PoLlin, Flora veronensis.

gioze de sangue, Trévise, SACcardo, Flora trevigiana.

(1) Comparez gallant, nom anglais de l'anemone hortensis, plante de la même famille. 
paparinicchia de ventu, Sicile, Cupaxi, IIortus catholicus, 1696.

russuliddu, Sicile, G. Buxci, Flora dei dintorni d'Avola (Dans Atte dell'

Accad, di scienze nat. di C'atania, 18.9-1857.)

piumétta, Parme, Malaspina, Voc. parm.

ogu de boi, Sardaigne du Sud, Moris, Flor'a sardoa.

cardinalin, pianta malanni, Tessin, Zeitschr.f. d. deutsche .Iythol. IV, 177. camomilla rossa, italien, selon MoRri, Diz. rumagn.

yallapozza, chegasangh', 111., romignol, Morru.

uy de perdiu, Minorque, Costa, Introd. "la Flora de Calal.

ull de perdiu, Vall de Nuria (Catalogne), Varreds, Catal. de la Fl. de V. salta-ojos, yota de sangre, espagnol, CoLmeiro, Diccion. 1871.

ollo de perdis, gallicien, Valladares.

rushcuté (= la petite russe), rommain, Сінас, Dict. daco-roman;

BRANDZA, Limba bot.

cocoshei (1), plur., roumain, ClнAC, Dict. daco-rom.

adonisröschen, ackerröschen, feuerröschen, teufelsauge, allemand.

schabab, eanton de Lucerne, Durheim, Schw. Idiot.

bluatstrïpfli, canton de Saint-Gall, Wartuaxx, P. z. St-Gall. Vollisbot.

blutströpfli, canton de Berne, Zeitsch. f. d. d. Mythol. IV, 177.

braune mädel, marienröslin, feuerrösel, rosa liamillen, Silésie, Pritzel.

schu'arzbraun mädchen, Hesse, IDEM.

ladderbleam, Schässburg (Transylvanie). IDEM.

füerfünlisken, Westphalie, LAxdors, Westfäl. Pflanzennamen. 1882.

bluetströpflan, toiflaugen, Carinthie, Zwaxziger, Verzeichn., 1888.

diüelsooge, bas allemand, Focke, Vollisth. Pflunzennamen, 1870.

füerooge, Frise orientale, IoE.r.

waild holieschblommen, allom. de Transylvanie, Fusz, Trivialnamen, ete. bruyneltekens, flamand, Dodoxaeus et Oudemans, Flora van Ned. A.de C.] duivelsoog, koollien-vuur, Flandre et Brabant. [A. de Cock.]

adonis flower, purple camomile, red camomile, red mathes, love-liesbleeding, anglais dialectal, Brittex et HoLl. Dict.

pheasant's eye, rose-a-ruby, anglais, Prior, Popul. names.

bloddroppar, gossen $i$ det gröna, suédois, Jexisex-Tusch .

brune piger, jomfruen $i$ det grönne, damois dialectal, IDEs.

rdeća mrvca, rudeća lamilica, planencic. ognjenice, serbo-croate, SUleK, Juyoslav. im. bilja.

(1) Cocosh en roumain signifie coq. La plante est ainsi nommée à cause de sa couleur rouge comparée à la crète de l'oiseau domestique. En France, dans quelques prorinces, on appelle le papaver rlocas coq, pour la mème raison. 
hlavàcek jarni, ohniček, tchèque, A. Müller, Wört. syn. nam. pavlyni otchi (= yeux de paon), petit russien, comm. par Tн. VoLkov. lialias viraig, magyar, Fusz, Trivialnamen.

ben na'aman, arabe algérien, MuxBr, Not sur les noms ar. des pl.

$n a ̂ b$ djemel (mot à mot dent de chameau, arabe de Constantine, PRAx,

Plantes de Constantine, etc. (dans Revue de l'Orient, 1850).

\section{AI)ONIS TER.VLLIS. (LINNÉ).}

1. Nons de CetTe plante.

aourignol. Snd-0uest dı Languedoc, Duboul.

spintc, cucoshel, roumain, Braxuza, Limba botanica.

bölımische christwur:al, böhmische nieswursel, falsche christun':el, allemand.

böhmische christwurz, Silésie, Маттuschка, 1779. [H. J.-T.]

gelle arschtguclien, Basse Autriche, F. HoEfEr.

lioe-ooghe (= œil de vache), flanand, Dobonaeus, Cruydt-boeck. 1. de L.] kasllöser, arontorpsros, aronsros. suédois dialectal, JENSSEN-Tuscir. troll-öjn, danois dialectal, IDE.I.

gorocvet, zeiji mak, serbo-croate, ŠLEk, Juyosl. etc.

scheltoi zvet (prononcez jeltoi zviet), russe, FALK, Beytrïge etc.

starodoubka, russe sibérien, PALlas, Voy. en Russie, III, 26.

horytzvit (= fleur llamboyante), petit russicn, comm. par TH. VoLког.

\section{USAGES.}

Die schwarze, bittre Wurzel wirkt scharf purgirend und emetisch und wird in den (namentlich franzœsischen) Apotheken oft statt der schwarzen Nieswurz gehalten. - Rosenthal, Synopsis plantarum diaphoricarum.

Le spintẹ est employé dans la mèdecine vétérinaire popul. des Rounains pour guérir certaines maladies des chevaux. - comm. par II. A. Gorover.

\section{ADONIS CUPANIANA. (GUSSONE).}

uy de perdiu, Baléares, Marès, Cat. des plantes des Baléares, 1880. a'llet el serduck, Malte, Delicata, Flor'a melitensis.

\section{ADONIS MGROCARPA. (DE GixdoLLE).}

nab-djemel (c. à d. dent de chameau), arabe algérien, Debeaux, Cat. des pl. du Boghar,

boû garoûna, arabe algérien, Duverrier, Les Touaregs.

a'in el buima (c. à d. œil de chouette), arabe de Cyrénä̈que, Ascherson, Pflanzen der Rolılf's Expedition (dans G. RoHLF, Kufra, 1881). 


\section{AIONIS DENTATA. (DELILE).}

schubbôtan, arabe de Tripoli, Ascherson, IvE..

\section{THALICTRU.I FLATTM. (LINXÉ), - LA RUE DES PRÉS.}

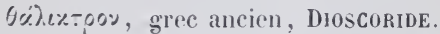

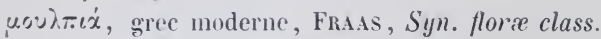

thatictrum, latin, PliNe, Hist. natur.

accipitrina, sophia, eruca geratina, latin du Moyen-Ige, DıEfexBAch, Gloss. lat. germ. mel. xtatis.

pigamum, ruta pratensis, saxifraga lutea, barba caprina minor, thalictrum majus, thalictrum, thalietrum (1) (sic), nomenclat. du Boyen-Age et de la Renaiss. Raunis, Pinax theatri bot., 16il.

rue des pauvres, f., rue des prés, f., rhubarbe des pauvres, f., fausse rhubarbe, f., pigamon, m., français.

mule dels prats, f., Pyrénées orient., Companyo, Hist. nat. des P. or. rubarbo saoubajo, f., Béziers, AzAis, Dict. des il. du midi. rubarbo salbatjo, Sud-0uest du Languedoc, Duboul.

raw, f., row dè prés, f., wallon, Feller.

persil des prés, Aube, Des Etaxgs; Gúvin, Statist. du canton des Riceys.

frenelle. f, Anjou, Desvatx, Flore de l'Anjou, verdemarco, Toscane, CaEsalpisus, De plantis, 1583. ruta di pralo, ruta saliatica, pigamo, erba pina, talillro, italien, TARGioni.

erba pinera, erba pignoeu, milanais, Baxf, Voc. mil.

ruda dels prats, ruibarbo aiels probes, Vall de Nuria (Catalogne), VAYREDA.

rutislior, ruta de livedç, roumain, Braxdza, Limba bot.

feld rude, anglo-saxon, Cockarve, Leechdoms, 1866.

mealow rue, false rhubarb, anglais.

fen rue, anglais, Prior, Popular names.

sophienkiraut, heilblatt, allemand.

krïtendill, krottendill, ancien allem., Diefenbach, Gloss.

grau bergmünnlein, sonnenwirbel, Silésie, Pritzel et Jessen, Volksn. d. Pfl.

(1) Ce nom dû originairement à une fausse lecture a été adopté par plusieurs botanistes du Moyen-Age et de la Renaissance. 
federbusch, Ulm, Idem.

valsche rhabarber, water-ruyle, poel-ruyte, flamand, DoDonaEus, Cruydtboeck. [A. de C.]

heilblad, hollandais, Oudemans, Flora. [A. de C.]

veldruit, poelruit, hollandais, Nemnich, Catholıcon.

troed y barcud, (= pied de milan) gallois, Hugh DAvies. [H. G.]

arrianllys (= herbe d'argent), gallois, Silvan Evans. [H. G.]

engjeruta, torriärksyrïs, iktegräs, suédois dialectal, JENNSEN-TUSCH,

Nord. plantenavne.

raenselyras, norvégien dialectal, IDFM.

linaep $i$ panden, froestjerne, danois dialectal, IDEM.

metiljlia, ljetna pavenka, kozlačica, serbo-croate, Šulek, Jugosl. im. bilja.

ruta, lithuaniell, JАсовт, Litauische Pflanzennamen, 1884.

rutka zolta, polonais, LINDE, Slown. jez. polsk.

wrzodowiec, stulis\%, polonais, Nemnich, Catkol.

sololucha trawa (= herbe à la scrofule), saplisa, russe, Falk.

zolotoucha, russe, Neunich, Catholicon.

vad virnantz, magyar, Nemnich, Catholicon.

angelma, finnois, Fellax, Index plantarum in Lapponiâ fennicâ lectarum, 1835.

rist-rabanduze-rohi, esthonien, WiEdemans, Ehstniches Wört.

\section{FOLKLORE.}

On dit que le thalictrum suspendu avec fleurs, feuilles et racines dans les chambres à coucher, ou fiché dans les berceaux, peut préserver les jeunes enfants de tout mal et accident ou de maléfices et que l'odeur est utile dans l'épilepsie.

Trad. de Dodonaeus, Cruydt-boeck. [A. de C.].

\section{THALIGTRLM MINLS (LINNÉ).}

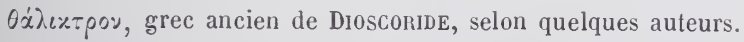

ruta pratensis tenuifolia, ruta pratensis minor, thalictrum tenuifolium, nomenclature du Moyen-Age et de la Renaissance, BauHin, Pinax theatri bot. 1671.

erba scopaia, ital., TARGIONI, Diz. botanico.

kleine wiesenraute, allemand.

graumündel, grau mennlin, Silésie, Pritzel et Jessen, Volksn. d. Pfl.

očobajka, serbo-croate, ŠLLEk, Jugosl., etc.

kozberet-el-habechy (c. à d. coriandre d'Abyssinie), kozberet-eth-thaleb. arabe, IBN-EL-BeIthar, Edit. Leclerc. 


\section{THALICTRIIM ALPINLYU (LINÉ).}

vélindisurt, lirossgras, jufismein, lrjostayras, krerkayras, Islande, Jensse.tusch, Nord. planten.

\section{THALICTRUM ANGUSTIFOLIU.M (LINAÉ).}

erba cipressa, ital., TARgioxi, Diz. botanico.

slutucha, tchèque, A. Muelder, Würt. syn. namen, etc.

talin, trambulika, mrkovec, serbo-croate, Šulek, Jugosl.

\section{THALICTRIM GLAUCUM (LINNÉ).}

ruda dos prados, galicien, Cuverro, Dicc. gallego.

\section{- TIIALICTRITI F(ETII)I.U LINAÉ).}

silfu, fu agrestis, martagon (selon quelques-uns), lat. du XVe s.. Cavos, L'opera salernitana, etc., p. 117.

valeriane sauvaige, f., français du XVes., IDEM, p. 117.

\section{TH.1LICTRLII AQLILEGIFOLIL.M (LINÉ).}

panatché (l), sud-ouest du Languedoc, Duboul.

panachèrés, f. pl., Luchon (Pyrénées), Jules SACAze.

colombine panachée, f., français, Gillet et Magne, Nouv. flore française.

\section{THALICTRIII CALABRICUII (SPRENGEL).}

adiäntu biancu $\left({ }^{2}\right)$, sicilien, Mortillaro.

\section{B.ATRACHII.1I FLLTTANS (Wingei). - LA RENONGULE D'EAU $\left.{ }^{3}\right)$.}

ranunculus palustris, ranunculus aquaticus, ranunculus fluviatilis, ancienne nomenclature, Bathis, Pinax theatri bot. 1671.

ranunculus aquatilis, nomenclat. de Lixié.

(1) Allusion à la fleur formant panache.

(2) Si vende presso noi falsamente per l'adianto bianco. (Mortillaro).

(3) Le rulgaire confond, sous les noms qui suivent, diverses espèces de renoncules aquatiques. 
ranunculus fluitans, nomenclat. de LAMARCK.

renoncule d'eau, f., grenouillette, f., grenouillette d'eau, f. bassin d'eau, m., bassinet d'eau, m., francais.

herbe aux grenouilles, f., env. de Domfront (Orne), c. par M. Aug. Chevalier. grapaoudino, f. Apt (Vaucluse), Coligrox, Flore d’Apt.

ranouncles, Gard, comm. par II. P. Ffsquet.

ranombe d'aiwe, blanc ranombe, ranombe di sanquiss, iebe di corauye wallon, Sesertier.

herbe à l'écrevisse, f., herbe à lu crabosse, f., crabosse, f., herbe à la moutelle ( ${ }^{1}$, f., Aube, Des Étaxgs, Noms pop. des pl. - HauteMarne, comm. par M. A. Daguin.

herbe aux égrevisses, f., Meuse, Labotrasse, Gloss. du pat. de la Meuse. grabosse, f., greubeusse, f., creubeusse, f., Haute-Marne, communiqué par M. A. Dagur.

erbo dei grapaou, erlo dei yranouio, Forcalquier, c. par II. E. PLauchid. fanes, trempette, f., Normandie Jorkt, F'. pop.

fanau d'itoué, m., Suisse romande. Vicat, Ilist. des pl. vénén. de la Suisse, 1776.

fanau d'aigue, in., Suisse romande, Derhein.

tirasseto d'aïgo, f., Var, Haxri, Cat. des pl. du Var. - Apt (Vaucluse), Coligxox, Fl. d'Apl.

brouille blanche, f., Bresse, Bossi, Statistique de l'Ain.

nego-fol, m., gascon, Azass ; Tarn-et-Garome, LAgrèzE-Fossat; Toulouse,

Touriox; Castres, Coczinié.

nego-foou, m., environs d'Avignon, Palux, Catal. des pl. du terr. d'Av. nayées, f. pl., mort chevaô, f., Mayenne, comm. jar un botaniste de la

Mayenne.

pihelé, m., piholè, m., picholè, m., pichali, m., pichâ-o-lè, m., dyènin, m., wallon, FELLER.

éperdriosse, f., herbe qui gratte, f., Méry-sur-Seine (Aube), Mérn. de la

Soc. d'agric. de l'Aube, 1863, p. 286.

marguerite de riviere, f., Aube, Des Etaxgs, loms pop. des pl. marguerite des rus (=m. des ruisseaux, f. Semur (Côte-d'Or', c. par

II. H. Marlot.

sainme (prononcez sain-me), f., Montbéliard, Coxtejeax, Gloss. du pat. bovairon, in., fribourgeois, Graxgier, Gloss. frib. morso di rana, italien, DuEz, Diz. it. franz., 1678. yerba lagunera, espagnol, Colsieino, Dicc. de los nombres.

(1) La moutelle est le poisson appelé en français loche. (Colitis barbatula). 
naprasnic, roumain, CıHAc, Dict. daco-rom.

sîm, m., sìm, m., Luxembourg, J. Weber.

lock, winterlock, flaslock, Alsace, Pritzel et Jessex.

haarkraut, wasser-flaclis, queis-flachs, Silésie, IDEM.

wasserfenchel, Berne, IDEM.

petersilienliraut, w'asser-anemone, Prusse occident., Treichel, Volkstl. IX, 1894.

flus:hähnchen, Prusse, Frischbier, Pr. Wört.

jâekelkruud( $\left.{ }^{(}\right)$, bords de la Weser, Focke, Volksth. Pflanzenn. der Heser. eel-beds, eel-u'are, anglais dialectal, BRITTEx et HoLl., Dict. of engl. plantnames.

rate, West Worcestershire, Chamberlaix, Gloss. of West-Worcestersh. u'ords.

reits, white croufoot, Shropshire, Jacksox, Shropsh. word-book.

fleann uisge (= le suiveur de l'eau) $\left(^{(}\right)$, lion na l'aibline (= lin de

rivière), gaélique écossais, Camerox. [H. G.]

neul uisge (= mage ou étoile d'eau), irlandais, CAMErox. [H. G.]

tuirchis fialhain (= renoncule saurage), irlandais, 0'RerLlY. [H. G.]

kupalnika, serbo-croate, ŠLEK, Juyosl., etc.

\section{R.ALYGLLL'S ACONITIFOLILS (LINAÉ). - LE BOUTON D'ARGENT $\left({ }^{3}\right)$}

groffe. f., Vallée de Cleurie (Vosges), Thiniat, La Vallée de Cl. - Vallée

de la Moselotte (Vosges), ChÉment, Quelques v'alleees vosg., 1861. Gérardmer, La Forge, (Vosges), Haillait.

pottas, plur., Ban de la Roche, H. G. OBerlix, Descript. du Ban de la Roclie, 1806.

bouton d’argent, m. (variété double cultivée). français.

belle pucelle de France (4), f. (idem), français, Vilworis, Fleurs de pleine terre, 1870.

boutoun d'argent, m. (idem), provençal mod., Lioxs.

broutou d'argent, m., Lauraguais, c. par M. P. FAG0T.

poumpoun d'argent, m., Luchun (Pyrénées), Jul. SaCaze.

(1) Weil die Pllanze Jucken erregen soll. (Focke).

(2) La renoncule d'eau vit en groupes nombreux et serrés dont les longues tiges flottantes suivent le cours de l'eau.

(3) Le vulgaire ne la connait guère qu'en tant que cultivée, à fleurs doubles.

(4) Ce nom, introduit rècemment dans la langue française, est la traduction de l'anglais fair maid of France. 
pounpoun d'argent, m.. Lot et Gar., c. par II. l'abbé L. Darur.

pimpoun d'argent, Sud-0uest du Languedoc, Debocl.

merle, canton d'Aime (Saroie), comm. par M. Marjollet.

merlon, m., Bas Valais, Gllliéros, Patois de Vionna‡.

merlot, m., Bex (Suisse romande), Bridel, Gloss. du pat. de la Suisse rom.

bouton d'argent d'Angleterre (la variété cultivée), français, Le bon jai-

dinier pour 1811. p. ¿10.

piè di cornacchia, ital., TARgrox, Diz. bot

boton de Francia, m., espagnol, Colmeiro, Dicc. de los nombres.

schïnes mädchen aus Frantireich, allemand, PRITzEL et JEss.

fair maid of France (la variété double), fair maid of Kent, anglais,

Brittex et Holl.

soläjlionge, rommegraes, norvéğien, JExssex-Tusch.

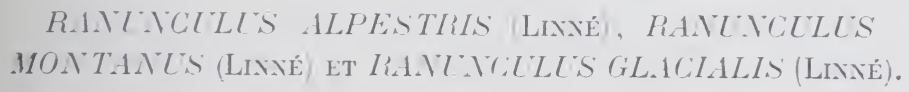

roys :

eirba de l'étaila, f., Suisse romande, Bribel, Gloss. du pat. de la Suisse. carlina, Les Ormonts (Suisse romande), DuruEim, Schueiz. Idiot.

camozèra, f., Valteline, Moxtı.

alpenhahnenfusz, allemand.

jügerkraut. Suisse allemande, Cuusius, Rarior. plant. historia, 1601.

feberkraut, Autriche all., IDEM.

ganshiess, Autriche allem., F. HoEFER.

FOLKLORE.

Im Berner Oberlande tragen die Gemsjäger den Alpenhahnenfusz bei sich, um sich gegen den Schwindel zu schüitzen. - Zeitsch. f. d. d. Mythol., IV, 175 .

R.1.TECLLLS ASIATICIS (1) LINAE - LA RENONCLLE DES JARDINS.

Fr:oxyı\%, grec anc. d'Hippocrate selon Rosexthal, Syn. pl. diaph.

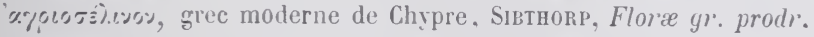

renoncule des jardins, f., renoncule des fleurisles, $f$, renoncule, f. français.

(1) D'une manière générale on donne aux autres espèces de renoncules cultivées les mèmes noms quau Ranunculus asiaticus. 
ranounculo, f., provençal mod., Lioxs, Végétaux utiles. renouncula, f., Basses-Alpes, Annales des Basses-Alpes, II, p. 8. ranuncle, f., Pyrénées orient., Conpayro, Ilist. nat. des P. or. renonculo, f., Toulouse, Toursos, Fl. de Toulouse.

renounculo, f., Forcalquier. c. par II. E. PLadcird. - Lauraguais, c. par M. P. F.ıот. - Lot-et-Crar., c. par M. l'abbé L. DARDr. reloncora, f., Menton, Axdrews, Voc. ment.

renonque, f., ernongue, f., ernonque, f., Valencienne', HEcart, Dict. rouchi. ranomb, raimonk, Spa, LEzAACK. Dict. des noms wallons des plantes.

renope, f., Verviers, Gravdgag.Mage, Dict. wall.

ranonke, f, ranompe, f., rénonlie, f., ralongue, f., wallon, Ines.

arnoncule, f., Saint-Pol (Pas-de-Cal.), c. par II. Ed. Eomoxt.

renoncule semi-double, $\mathrm{f}$, semi-double, f. la renoncule à laquelle il reste quelques ítamines et par conséquent la faculté de porter des graines), français des jardiniers, Bon jarlinier pour 1805 .

renoncule-ilouble, renoncule-pivoine, $\left({ }^{1}\right), \mathrm{f}$., celle dont toutes les étamines ont éti changíes en pétales et par suite inféconde), français des jardinier's, Bon jardinier pour 1805.

rosellini di giardini, rosellina, ranuncolo, ranuncolo persano, italien. ranincolo, napolitain, Gusumpaur.

ramuncole, Salnces, EANDr. Stat. della prov. de Saluzzo.

arnonncoula, piémontais, CAPELlo, Dictionn. piémontais.

arroncola, piémontais, ZALLI, Dis. piem.

naroncolo, Venise, Boerio, Diz. d. dial ven.

noroncol, milanais, Baxfi, loc, milan. - Parme, Malaspre, Voc. ranunculu, Sicile, Cupsix, IIortus catholicus, 1696.

narunculo, Vérone et Lombardie, PoLliNi, Flora veronensis.

rusètta, Bologne, Coroxeu-Berti ; romagnol, Morri.

rusella, musellu, Sicile, Macaluso, loc. sic.

rôseutt d'Olanda, Plaisance, Bracciforti, Flora piacentina, 1877.

ruslën d’Ulanda, naröncal, romagnol. MorRi.

uocchio de roje, napolitain, Gusumpaur.

giganti (= ranunculus axiaticus flore pleno rubro), italien, TARgroni. ranunculos dos jardins, borboletas, flores de quaresma, portugais,

внотев0, Flora lusitanica, 1804.

(1) Aussi appelée renoncule d'Afrique. Il y en a 5 variétés : la pivoine rouge ou rouma; la séraphique d'Alger, couleur jonquille; le sonei doré ou merveilleuse, couleur de suuci duré, cour vert; et le turban doré, rouge panaché de jaune. - Bon jardinier pour 18:7, p. 608. - Le Ranunculus ufricanus est peut-être une espèce diffẻrente du ranuneulus asiaticus. 
marimoña, espagnol, Colmeiro.

fransasilla, catalan, Costa, Intr. ì la Fl. de Cat.

ranunkel, gartenranunkel, türkische ranunkel, allemand.

ranunlielchen, Luxembourg, J. WeBER.

arunkele, Alsace, hirschleger, Fl. d'A'sace.

renonkels, flamand. [A. DE C.]

edaskia. basque [J. V.]

saghlil, arabe de Cyrénaïque, Ascherson, Pflanzen der Rohlf's Expedition, 1881.

heranounghe, arménien.

zérène, turc.

On trouvera les noms français des variétés de Renoncules cultivées dans :

10 Vallot, Hortus regius, 1665. in-fol., p. 149-153.

2. D'Ardène, Traité des renoncules, Paris, 1746, in-8.

Quelques auteurs prétendent que cette plante, originaire de l'Asie mineure, a été introduite en France par les Croisés, du temps de Saint Louis: mais il paraît que ce ne fut que sous Malımet IV que l'ou se procura les belles variétés doubles que nous possédons aujourd'hui de cette espèce dont les racines appelées griffes servent à les perpétuer. Mérat. Dict. de mat. médic., 1834

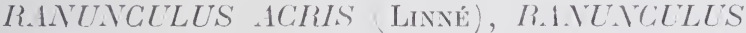 REPENS (LINNÉ) ET RANUTYLLLS BLLBOSLS (LINNÉ). LE BOUTON D'OR.}

NOMS :

Ces trois espèces sont ordinairement confondues sous les noms suivants $\left(^{1}\right)$ :

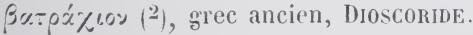

ranunculus, latin.

batrachium, polyanthemum, latin du $4^{\mathrm{er}}$ siècle après J.-C., Scribonius LARgus, cité par Mever, Geschichle der Botanil.

batrachium, pes corvinus, pes gallinaceus, gallipes, latin dâ moyen âge, Diefenbach, Giloss lat. germ. med aet.

(1) Le vulgaire confond encore sous ces mêmes noms un grand nombre d'autres espèces de renoncules, dont la fleur est jaune.

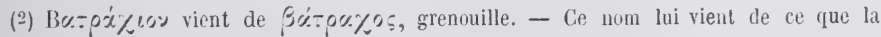
plupart des espèces du genre Ranunculus habitent les lieux marécageux. 
ranunculus pratensis erectus acris (= Ranunculus acris, L.) anc. nomenclat., Baunin, Pinax theatri bot., 1671.

bassin, m., français ancien et moderne. - Champagne. - Bourgogne. Orléanais.

bassinet, m., français anc. et mod. - Champagne. - Bourgogne. Orléanais. - Artois.

bassin d'or', m., Bresse châlonnaise, L'Intermédiaire, 1, 18 '.

bachinet, m., Oise, Graves, Cat. des pl. de l'Oise. - Normandie, Jonet, Flore pop. - Boulonnais, c. par M. B. DE Keruerié. - Pas-de-Cal., c. par M. Ed. EDMont.

bassinot, m., Côtes-d’Or, Roren, Flore de la Côte-d'Or. - Bulgnéville (Vosges), Haillant. $F l$. pop. des $\Gamma^{\circ}$.

bassignot, m., Haute-Marne, comm. par M. A. DAGUin.

bassingnol, m., Côte-d'Or', comm. par Il. H. MaRLot.

bèssingnot, m., Forêt de Clairvaux (Aube), Baudourn, Gloss.

bèssimot, m., Ronceux (Vosges), Haillant, Fl. p. des $V$.

clair bassin, m., Anjou, Desiaux, Fl. de l'Anjou. - Environs de Pithiviers (Loiret), c. par M. J. PoQUET.

gobelet, (') m., ancien français, LiḱBault, Maison rustique, Edit. de 16in8, IV, 4, P. 480; OudIN, Trésor, 1660.

gobelet d'or, m., Loiret, c. par M. J. POQUET.

gobelet du diable. m., Noisy-le-Sec (Scine), rec. personnellement.

chandiòre du diable. f., feu d'enfer, m., Franche-Comté, Dartois, Etude des pat., 1850.

yodinot, m., Meuse, Labourasse, Gloss.

pot an beurre, m., poinsot, m., chouquet, m., herbe a la tache, f., Normandie, Jonet, Fl. de $N$.

pot de chambre, m., pot d'étain, m., environs de Vire (Calvados), comm.

par II. J. Counaye du PARc.

grenouillette, f., ancien franç. Pinaeus, Historia plantarum, 1561.

grenouillet, m., ancien franc., Proprietez des simples, 1569, p. 41.

bouton d'or, m., renoncule des prés, f., grenonillette, f., français.

ranoncule, f., bacin, m., anc. franc. Philiatre, Tresor des remedes, 1555, p. 122.

boton d'or, m., Haillant, Fl. p. d. Vosges.

bouton d'or, m., sud-ouest du Languedoc, Dubocl.

boton d'aur, m., Spa, LezAACK, Noms wallons.

bouton d'ouai, m., Vallorbes (Suisse rom.), Vallotton-AUbent.

(1) DUEz, 1664, ne donne le mot qu'au pluriel. 
boutoun d'or, m., provençal mod.; languedocien.

boutoun d'or, m. jaounoun, m., erbo dei mandiant (1), f. Forcalquier, c. par M. E. Plauchud.

broutou d'or, m., Lauraguais, c. par M. P. FAGoT.

bouton de Saint-Jacques, m., Pithiviers (Loiret), comm. par M. L. MALON. beul' d'ô, f., Bourg (Haute-Marne), rec. personnell.

pimpon d'or, m., Mayenne, comm. par un botaniste de la Mayenne.

pimpoun d'or, m., Bas-Quercy, comm. par I. J. DArmand. - Sud-Ouest du Languedoc, Duboul.

pipoun d'or', m., (pipoun signifie bouton) Luchon (Pyrénées), J. SACAzE. poumpoun d'or', m., Saint-Pons (Hérault), BARThĖs. - Lauraguais, c. par

M. P. Fagot. - Languedoc, Duboll.

pounpoun d'or, m., pinpoun d'or, m. Lot-et-Gar., c. par M. l'abbé L. DARDY.

bouton d'or (la variété à fleurs doubles), Ramecourt (Pas-de-Cal.), c. par M. Ed. Ediont.

pompon d'or, m., Saint-Pol (Pas-de-Cal.), c. par M. Ed. Edyont.

gannet, m., Pays de Bray (Normandie), Decorde, Dict. du pat. du pays de

Bray. - Normandie, JoRET, Fl. p.

jaunet, m., ancien français $\left({ }^{2}\right)$; normand.

jauneau, m., Haut-Maine, Montesson, Toc. du Haut-Maine. - Injou,

Desvaux, Flore de l'Anjou.

jauniot, m., Normandie, Jonet, Fl. pop.

jaounoun, m., env. d’Avignon, P.ıus, Cat. des plantes. - Apt (Vaucluse), Colignon, Flore d'Apt.

gane d'œu, m., Saint-Pol (Pas-de-Cal.), comm. par II. Ed. Ednont.

rouge bouqua, m., (Ranunculus acris), Vagney (Vosges), Haillant.

pâquerette jaune, f., anc. fr., DEcaIsne, Catalogue.

fleur de lait jaune, f., gallo du Morbihan, recueilli personnallement.

fleur au beurie, f., burre, f., Mons, Sigart, Gloss. montois.

erba de birro, f., fllau de bûro, f., Suisse romande.

(1) Ainsi appelée parce que les mendiants s'en serrent pour se faire des plaies sur le corps et ainsi exciter la commisération. E. P'L.

(2) On trouve ce mot dans Saint-Amant, La Rome ridicule, poème burlesque de la fin du XVII siècle :

Là, mille robustes Carites

Folàtrent sur l'émail d'un pré

Agréablement diapré

De jaunets et de margueri'es. 
erbo de buri (1), f., Briangomais, comm. par M. J.-A. Chabrand.

beûrin, m., Avesne-lès-Aubert, près Cambrai, recueilli personnellement. vilette ed' bûre, f., environs de Cambrai, rec. personn. gône bure, m., Isbergntes (l'as-de-Cal), c. par .I. Ev. Evмoxt. pied de coq, $\left({ }^{2}\right)$, m., anc. frans. - franç. mod., Bon jardinier pour 1843. pie coq, in., Le Mans, Mauxi.

patte de coq, f., français (de Bretagne?), LE (ionidec, l)ict. francaisbretom. [E. E.]

pas de coq, m., normand, JoRET.

piéco, m., Anjou, Destaux. - Haute-Bretagne, conm. par M. P. SÉBllot. piecou, m., environ d'Albertville Savoie', rec. personn.

piècour, m., Anjou, Desvaux. - Mayenne, c. par un bolaniste. - Vern

(llle-et-Vilaine), rec. personn.

pico, m., environs d'Amboise, rec. personn.

patte de jô, f., Ineuil (Cher), ree. personn.

piepon (3), m., anc franc., Pinaecs, 1561. - Morvand, Chambure. -

Doubs, Bendquer. - Jura, Monsier, Vocal). du Jura (dans Mém. de

la Soc. des antiq. 1823). - Yonme et Loiret, c. par M. J. Poquet.

piapu, m., Suisse romande, Vicat, Plantes vénéneuses de la Suisse;

Derheim, Idiot.

piapô, II., vandois, Callet. - Suisse rom., Durieim; Bridel.

piapor, m., lyonnais, I'uitspele. - Aime (Savoie), e. par M. Marjollet.

- Albertville (Satoie), F. Brachet.

pipor, m., lyonnais, Putspelo.

piapoeu, m., Albertville (Savoie), F. Brachet.

jiple, m., Arbois (Jura), comm. par II. H. DoRBon.

piape, m., pipe, n., Jura, Movier, Гoc. du Jura.

pipon, m., Oise, Graves; et Ammuaire de l'Oise, pour 1831.

piepoin, m., dauphinois, Putspell.

pipou, m., anc. franç., SEs. CoLIn, Onzieme lirre de Trallian traitant des gouttes, 1557, p. 165. - Montret (Saône-et-Loire), Gaspard, Notice sur Montret, 1866.

pipio, m., Auneau Eure-et-Loir), rec. persomn.

pipo, m., Chaumont (Oise), Frion, Descr. de Chaumont, 1867.

pieipo, m., La Hogue (Manche), J. Fleuri, Essai.

(1) Dans le Briangonnais les fleurs de cette plante servent à orner le beurre, surtout le jour de la Saint Jean. (Comm. de M. Chabrand).

(2) La plante est ainsi appelće à cause de la forme de la feuille.

(3) Cette forme correspond au latin fes pulli, pied de coq. 
pied-bot, m. (par fausse étymol. pop.), Eure, RoBin.

pipô, m., pibo, m., piepo, m., Normandie, Joret.

pipolet, m., picard, Corblet. - Somme, comm. par II. H. Carnor.

pied de poule, m., Centre, Jaubert. - Normand, Joret.

$\mu \hat{\imath} d$ ' pouye, wallon, Feller.

pourpier, (') m., anc. franc, La Maison rustique, XVIe s. - Doubs,

Beauquier, Voc. du Doubs.

porpier, m., Moussey (Vosges), Tranqueville (Vosges), Halldant Fl. pop. des $V$.

pourpie, m., Meuse, Labourasse, Glos. du pat. de la M. - Lemmecourt et Totainville (Vosges), Haillaxt, $\mathrm{Fl}$. des Vosges.

porpie, m., Mortagne (Vosges), IDEM.

poupiè, m., Jura, Beaunuer, Voc. du Doubs.

popiè, m., Ban de la Roche, H. G. OberLin, Description du Ban de la Roche, 1806.

poûpî, pôpî, wallon, FELLER.

poupeïe, m., Verviers (Belgique), LEJEune.

pas d'agache, m., env. de Samers (Pas-de-Calais), c. par II. B. DE KerhervĖ. pas de corbin, m., Normandie, JoRET, Fl. pop.

pied de corbeau, m., anc. franç., Duchesse, De stirpibus, 1544; Evon.

Philiatre, Tresor des remedes, 1555, p. 192.

pied de rourbin, m., franç. du XVIe siècle, ILatson rustique, XVI siècle. patte de loup, f., Injou, Desvarx. - Normandie, Joret.

pas de loup, m., anc. franc., Maison rustique, XVIe siècle.

pèe de loup, m., Nogay (Béarn), Lespr.

pè de lou, m., Sud-0uest du Languedoc, Duboul.

pôcre de loup, f., Bressuire (Deux-Sèrres), LaLanne.

potteloube, f., poiteviu, LÉvrier. - Chef-Boutonne Deux-Sèvres), BEAUchet-Filleau. - Anjou, Desvaux. - Charente-Inférieure, c. par

M. E. Lemarié.

paoutelou, f., Libourne (Gironde), c. par II. L. Durand-DÉGrange.

paoutolouvo, f., Saint-Germain (Lot), Soulié.

paoutolouo, f., Cantal, c. par M. J. MaLveziN.

paoutolobo, f., Aveyron, Varssier.

paoutoloubo, f., Ussel (Corrèze), G. de Lépinay.

pato de lou, f., lontipaoudos, f. pl., Saint-Pons (Hérault), Barthès.

loupipaoudo, f., Sud-Ouest du Languedoc, Duboul.

(1) Ce mot correspond au latin prlli pes, tandis que les furmes précédentes représentent pes pulli. 
loupaouto, f., Tarn et Garonne, LAgrezz-Fossat. - Correze, Béronie. Tulle, G. de Lepixar. - Cantal, c. par M. J. Malvezin, - Sud-Ouest du Languedoc, Durocl.

lipaouto, f., Brive (Corrèze), G. de LÉrsas.

loupateix, Ussel (Corrèze', G. de LÉriNaY.

pliante-loube, f., Denx-Sèrres, comm. par M. B. Souché.

pas de lion, m., pied de lion, m., padrion, m., normand, Jonet.

pas de chat, m. Domfront (Orne), c. par M. Aug. Chevalier.

pé de gat, m., béarnais, Lespr. - Sud-0uest du Languedoc, Duboul.

pied de chat, m., patte de chat, f., pied d'oie, m., patle de crapaud, f.,

Normandie, JoRET, Flore pop.

pied coulin, m., poite, f., Charente-Inf., comm. par M. E. LemarıĖ.

patte de raine, f., Lisicux, Dubors et Travers, Dict. du pat. norm.

pè de pourin, m., Libourne (Gironde), c. par II. L. DuraND-DÉgraxge.

poterotte, f., Montbéliard, CoNtesean, Glossaire.

lagaino, f., Gard, comm. par M. P. FesuUet.

laghéino, f., Anduze (Gard), Viguer.

lagagno, f., lagaigno, f., Sud-0uest du Langued., Duвoul. - Toulouse,

Tocrion.

mal d'els, m., Sud-0nest du Languedoc, Duboct.

pleur-yeû, m. (c.-à-d. чui fait pleurer les yeux), Romorantin (Loir-et-

Cher), rec. personncll.

mort aux vaches, f., Doubs, BEAuquier.

crebo-bioou, Saint-Pons (Hérault), BarTHĖs.

mort-cheval, Anjou, Desvalx.

més de maï, m., Var, Avic. - Bouches-du-Rh., Villexeure. - Basses-

Alpes, Annales des B.-Alpes, II, p. 14 et 16.

cocu $\left({ }^{1}\right)$, m. Clairraux (Aube), Bacdocin.-Environs de Cambrai, rec. pers. coqueî, m., Plancher-les-Ilines (Haute-Saône), Poulet.

phette é lé, f., Verviers (Belgique), LEJErase.

pillate è lét, f., Liège, c. par M. 0. CoLson.

aîuî, m., pihotte è lèl, f., wallon, SeMeritier.

cônet, m., cosmuau, m., Mašenne, c. par un bot. de la Mayenne.

tçassè, f.. Issoire (Puy-de-Dòme), comm. par M. J. Bareire.

colon, m., (Ranunc. acris), Bainville (Vosges), Haillast.

paubran (mot d'origine bretonne, voyez ci-dessous), gallo des Côtes-du-

Nord, Ernault, Revue cellique, V, 223.

(1) Les premières fleurs du printemps, qui paraissent au moment oủ le coucou ou cocu commence à chanter, sont souvent appelées coucous ou cocus. Nous en verrons nombre d'exemples par la suite. 
batrachio, italien, TARGIONI.

tazza, bacinella, ranuncolo, italien, Lexicon italico-latinum, Taurini, 1648, p. 109.

boton d'oro, Trévise, Saccardo. - lombard, Pollini.

pè d' gall, Parme, Malaspina.

pè d'oca, Plaisance, Bracciforti.

pè di nibbio, lombard, Scannagatti.

pè de nibbi, milanais, Gherubini.

pi d' nébbi, romagnol, Morri.

pè d'oc, cascadent, Piémont, Annales de l'Observatoire de Turin, 1810, p. 56 .

pè d'èsen, Modène, Riccardi, p. 60.

vesciolíni, Abruzzes, Finamore.

giaccaria, Saluces, Eand, Statist. d. prov. di Saluz:o.

pedenebol, Brescia, Melchiori, Voc. bresc.

pirl di crott, Frioul, P'IRoNa, Vocab. friulano, 1871.

ranuncla, Engadine et Grisons, Dunhers, Schweiz. Idiot.

ranunculu, erba de ranas, Sardaigne, MoRIs, Flora.

boton de oro, espagnol et catalan.

yerba belida, espagnol, J. Victor, Tesoro de las tres lenguas, 1609 ;

Minsiev, Dict. in spanish and english, 1623; Colmeiro, Dicc. de los nombres.

guiabella, espagnol, Missheu, Dict. in spanish, $16 \succsim 3$.

rarunchiu, flóre broscésca (= fleur de ğrenouille), piciorul-cucoshului, bujorel, roumain, BRAIDZA, Limba botanica.

gelle schmaltablumen, allemanl, EBER et PEucEn, Appellationes quadruperlum, etc. 1556.

abbeis, teufel-abbeis, Autriche allem., Cuusius, Plantarum rar. hist. 1601. froscfusz, rappenfusz, gleiszblumen, moyen haut allem., DiefenвAcI. hahnenfuss, lirähenfuss, schmalzblümchen, ankelblume, viesenhahnfusz, butterblume, allemand.

botterblum, pelpes, delpes, echelskraut, Luxembourg. J. WEBER.

teufelsabbiss, Autriche allem., Pritzel et Jessen, Folksn.

pfännlein, Souabe, IDEN.

pfändle, Memmingen, IDE.I.

gauseblïmkes, Westphalie, LANDors.

galliseli, glizerli, canton de Saint-Gall, Wartuans.

boglahrcher, all. de Transylvanie, Fusz, Trivialnamen.

schmal:bleaml, zengerblüemel, zengerliraut, zengearreasl, zengerrosen,

Carinthie, ZwaNziger, Verweichniss.

hanevoet, hanepoot, boterbloem, hollandais et flamand. [A. de G.]. 
polpit, Denderleeuw (Flandre orientale), c. par M. A. DE Cock. lodeu'orte, ramnes fot, anglo-saxon, Cоскаух, Leechdoms, etc. croufoot, crowflower, anglais.

gold cup, Somersetshire, Jenvixgs, Obs. on some of the dial. of England. golden cup, gilly cup, West Somerset, Elworthy, W. Som. word-book, 1886.

king cup, Deronshire, Friesd, Gloss. of Deronsh. - Lothian (Écosse),

JaMIESox, Etym. dict. of the scott. lang., 1882.

butter-rose, Devonshire, Friesi, Gloss. of Dev.

bassinets, anglais, Prion, Pop. names of $p l$.

craw foot, craw tae, locker, Écosse, Janiesox, Etym. dict.

crazy, crazey, Wiltshire, Prior, Pop. names. - Glocestershire, Marshall,

The rural economig of Glocestersh., 1796.

yallow call, yallow cup, île de Wight, Loxg, Wight dialect.

butter creeses, anglais dialectal, Brittex et Holland, Dict. of the engl.

plant-names.

eggs and lutter, IDEM.

butter daisy, IDE.I.

horse gold, gold crop, IDEM.

goldy knol, limy's linob, king's col, IDE. .

blister plant, IDEM.

rams-claw's, Somersetshire, Jexings, On some of the dialects of England. crafang y fran = griffes de corbeau', gallois, J. DarIes, 1632. [H. G.]. cearban feoir (= lierbe des guenilles), gaélique écossais et irlandais, Camerox. [H. G.]

olbrain (= empreinte de corbeau), gallois, J. WaLters. [H. G.].

chw'ys Mair (= sueur de la Vierge Marie), gallois, J. Walters. [H. G.]

egyllt, gallois de Llanrust, John WiLliass. [H. G.].

pau-bran (= patte de corbeau), breton, Nomenclator; et GRÉG. DE RoS-

TRENEN. [E. E.j

paô-brân, LE GoNidec ( $\left.{ }^{1}\right)$. [E. E.].

paubran, breton de Vannes, Dict. de l'A*** [E. E.].

soley, brennisúley, Islande, Jexisex-Tusch, Nord. plantenarne.

svujna-kuannur, Iles Feroë, IDEM.

solïga, solmöjegräs, smörblomster, majblomster, suédois dialectal, IDEM. kragefod, hanefod, danois dialectal, IDEM.

(1) Cet auteur donne encore à tort, comme synonyme de renoncule, dans son dict.françaisl,reton, le mot c'houervizon qui veut dire pissenlit et au dict. br.-fr., il signale une confusion inverse de LE PELletier qui avait donné paubran = "pissenlit, " suivant d'autres $=$ " marguerite. " [E. E.] 
smgrleger, gigtblomster, singtblomster (= fleur de beurre), danois dialectal. [H. F. F.].

jabokrel, żabjak (= plante des grenouilles), iskrića (= étincelle), izjed

(= corrosif), maslice, zlatica, zlatenca (= dorée). ¿̇eżara, serbocroate, ŠULEK.

slatitza, Carniole, LINDE, Slown. jez. polsk.

vrâtich, croate, Idem.

jaskier, polonais, Idem.

srocyna pazora, żoru̇, wende, Schulenburg, Wendisches Volksthum, 1882. jâbnik (= qui a rapport aux crapauds), russe. [Th. V.]

kouriatcha slipotá (= la cécité des poules; comparez ci-dessus p. 23, en note), petit russien. [Th. V.].

wedrynes, gaidylai, yaidpil'szczai, lithuanien, JАсовг, Litauische pflanะeun., 1884.

gaila pehdas, letton, SteNder, Lettisches Lexikon, 1789.

gaila kahjas, letton, Cumaxx, Lett. Wört., 1880.

narrililled, esthonien, Fellaras, Index pl. fenn., 1835.

tullikad, esthonien, Hupel.

söbija-rohi, näri-lille, esthonien, WiEdMan, Ehstn. Wört.

woi kuklia, finnois, Fellyax, Index plantaruin fenn., 1835.

beka fiu, magyar, Cuusius, Rar. plant. hist. 1601.

réti béliaviray (Ranunc. acris), magyar, Fusz, Trivialnamen.

roubeyya ote, turc, Barbier de Metinad, Dict. tuic français.

2. LOCUTION.

Jaune comme ein bèssingnot.

Forêt de Clairvaux (Aube), Baudors, Gloss.

3. USAGES.

Pour échapper à la conscription les jeunes gens se font au moyen de compresses de piépou (ranunculus acris), des ulcérations dangereuses qu'ils gardent quelquefois toute leur vie.

Còte-d'Or, comm. de II. H. Marlot.

Les deux plus grosses radicules de ces plantes, appliquées sur le pouls ont une singulière vertu pour chasser la fièvre... Si c'est un homme qui a la fièvre, liez ces radicules sur le pouls gauche, ou intérieurement sur le pouce gauche; pour une femme, sur le pouls droit ou le pouce droit. Laissezles pendant 9 jours, sans y toucher.

Trad. de Dodonaeus, Cruydt-boeck. [A. DE C.]

Le suc de la feuille est employé contre les verrues.

Lot-et-Garonne, comm. par l'abbé L. DARDy. 
Les fleurs du bouton d'or (ranunculus acris, var. à fleurs doubles), écrasées et appliquées sur les nids d'agaches (cors aux pieds), les font rapidement disparaître.

Ramecourt (Pas-de-Calais), comm. par M. Ed. Edmont.

\section{FOLKLORE.}

1. On répand les fleurs des renoncules des champs, dans la nuit de la Saint-Gengoult, devant la porte des maris trompés.

Forèt de Clairvaux (Aube), Batdors, Glossaire.

2. On empêclıe les enfants de toucher à ces plantes caustiques en leur disant que cela ferait périr les poulets et les oisons de la maison.

Ineuil (Canton de Chateauneuf, Cher), recueilli personnell.

La renoncule fait, dit-on, pisser au lit les enfants qui la portent à la bouclie.

Environs de Liège, c. par M. 0. Colsox.

3. Les ménagères qui veulent avoir du beurre bien jaune en été doivent mettre dans le pot au lait les trois premières fleurs de renoncule qu'elles trouvent au printemps.

Danemark. [H.-F. F.].

Si en touchant le menton de la ménagère avec une renoncule, il y reste des traces jaunes, elle réussira son beurre dans le courant de l'année.

Danemark. [H.-F. F.].

Pour le mal de dents, on attache une fleur de renoncule au pouce gauche au moyen d'un fil non blanchi, enroulé trois fuis; il s'élève une pustule et le mal de dents est gruéri.

Suède. [H.-F. F.] Comparez ci-dessus, p. 11.

Si l'on reut aroir du bon beurre toute l'année, il faut manger la première renoncule aperçue au printemps.

Suède. [H.-F. F.]

\section{RANLNGLLLS REPE.NS. (LINNÉ). - LA RENONGULE RAMPANTE}

$$
\text { 1. - NOMS DE CETte plante : (1) }
$$

ranunculus hortensis, ranunculus vinealis, ranunculus pratensis repens hirsutus, ranunculus pratensis reptante cauliculo, ranunculus polyanthemos, ancienne nomenclature, BAUнг, Pinax theatri botanici, 1671.

(1) Quand on ne la confond pas avec les ranunculus acris et arvensis. 
ranunculus dulcis, ranunculus mitis, anc. nomenclature pharmaceutique.

Rosenthal, Synopsis plant. diaphoric.

renoncule rampante, f., français savant.

aouriflan, m., Bas-Quercy, c. par II. J. DAYMARD. - Tarn-et-Garonne,

Lagreze-Foss. - Sud-0uest du Langued., Deboul.

aouruflan, m., Tarn (?), Poumarède. - Montauban, Gaterau.

aouriflam, m., environs d'Arignon, PALUn.

aouruflam, m., gascon, AzAis.

oouriflon, m., Saint-Germain (Lot), Soclik.

fraisier sauvage, m., fraisier de grenouilles, m., Loiret, c. par M. Ponuet. fresiè salbadge, m.. Saint-Pons (Hérault), BarThès.

vuide grange, persn, Oise, Innuaire de l'Oise pour 1831.

chasse, f., Centre, Jaubert. - Allier, c. par M. C. Bourgougnon.

pourpier sanvage, m., Clairvaux (Aube), Baudouin. - Haute-Marne, c.

par M. A. DAguin.

porpier, m., pourpier, m., Montargis, (Loiret, c. par M. L. MaLoN.

pourpien, m., galle, Haute-Mlarne, c. par II. A. DAguin.

trainasse, f., Centre, Javbert.- Haute-Marne, c. par M. A. Daguin.

renounculo tirassièro, f., Forcalquier, c. par II. E. PLAuculd.

violons, m. pl., Manche, c. par M. J. Colraxe du Parc.

esperoun, Sud-0uest du Langued., Duboul. - Luchon (Pyrénées),

\section{J. SACAZE.}

apit salbatje, m., pato d'aouco, f., Sud-0uest du Languedoc, Duroul.

herbe a la tire-youtte, f., Côte-d'Or. c. par II. MarLot.

din d'tchin (= dent de chien), flate di vatche, f. (= bouse de vache),

wallon, FELLER.

lompaouto, f., Areyron, VAYssier.

paoutoloubo, f., languedocien, c. par M. H. FAU.

pie de pigeon, m., anc. franc., Decalsne, Gatal.

piapau, m., Suisse romande, Bridel.

piépo, m., pié de po, m., environs de Maintenon (Eure-et-Loir), c. par

M. Gabriel.

piépou, m., Châtillon-sur-Seine (Côte-d'0r), c. par M. F. Daguix. - Semur

(Cùte-d'Or), c. par M. H. Marlot.

piépou traçant, m., Aube, Guenis, Statist. du cantou des Riceys, 1852.

pas de chat trainant, m., enr. de Domfront (Orne), c. par M. Aug. CHE-

VALIER.

pied de chat, m., Orne, LETACQ.

patrasse, f, béarnais, LESPY.

limaouco, f., meliaouco, f., mihiaouco, f., Gard, c. par II. P. FEsquet.

brancosin, milanais, BANFI, Voc. milanese. 
pedochi, tremolini, momolini, supette, Vérone, Pollini.

pè corvin, Frioul, PiroNa, Vocab. friul.

pataló, portugais, GRISLEY, Viridarium lusitanicum, 1660.

patelo, galicien, Cuverro, Dicc. gallego.

strupfe, Autriche allem., l'Ritzel et Jess.

grahfuss, graufuss, Eifel, Wintgen, Vegetation der Eifel, 1865.

gromes, Luxembourg, KoLTz, Flore du Grand-Duché, 1873. - Eifel,

Wirtgen, Vegetat. der Eifel.

krobes, grôfoss, gôfoss, Luxembourg, J. WEBER.

wetzel, Trèves et Mayence, PRITz. et Jess., Vollisnamen.

feldzonga, Basse Autriche, HєFer, Wört. d. niederöst. Pfanzenn.

marks, Prusse, Frischiser, Preuss. Wört.

meg-many-feets, granny threads, hod-the-rak (= hold the rake), anglais

dialectal, Britten et Holland, Dict. of engl. plant-names.

brigheag (= yellow), gaélique écoss., Cameron. [H. G.].

fearban, bairghin (= pilgrim's habit), bairgin, irlandais, Cameros. [H. G.] wis:tligjos, lithuanien, JACoBr, Litauische Pflan:ennamen.

woi liukika, finnois, Felliax, Index plantarum fennicarum. 1835.

tullikad, esthonien, IDEM.

\section{2. = DICTONS RELATIFS A CETTE PLANTE.}

Cetle plante est très difficile à extirper d'un terrain. Pour la faire périr, il ne suffit pas de l'arracher, il faut encole la secouer, d'où le dicton :

Lo lompaouto

Quoud ouon lo trago

Noun s'en chaouto,

Se l'ouon lo brandis

Ne péris.

(La renoncule rampante, si on l'arrache, elle s'en moque; si on la secoue, elle en périt.) Aveyron, Varssier, Dict. du pat. de l'Aveyron.

Dans le Languedoc, selon Poumaréde, Dict. des termes usuels, on dit à propos de la même plante :

Sé mé brandissés

Mé périssés.

Difficile à détruire comme le piépou. - Semur (Côte-d'Or), c. par M. H. MARLOT.

Ce sont de mauvais prés, il n'y pousse que du piépou. - IDEM.

En Suisse on considère comme une terre excellente celle où la renoncule rampante croît en abondance : 
Einke iô cret le taconnet

Laisse lo à kouï l'est,

Einke iô cret le piapau

Atzìta-lo se te pau.

(Le terrain où croît le tussilage, laisse-le à qui il appartient; celui où croìt la renoncule rampante, achète-le si tu peux.) - Suisse romande, Bridel.

\section{RANUNGULUS BULBOSLS. (LINNÉ). - LA RENONGULE BULBEUSE,}

\section{1. - NOMS DE CETTE PLANTE.}

ranunculus tuberosus, ancienne nomenclature, Baunis, Pinax theatri botanici, 1671.

renoncule bulbeuse, f., français savant.

pied de corbin, m., franç., BARBeU-Dubourg, Le botaniste français, 1767. [Ed. Eds.]

grenouillette, f., rave de saint-Antoine, f., franç., LAMARCK et CANDoLle. erbo dé sént Antôni, f., Lot-et-Garonne, c. par M. l'abbé L. Dardy.

herbe aux douves, (1) f., Aube, Des ÉTAxgs, Noms pop. des pl. de l'Aube, 1844.

jaune bouqua, m., Vagney (Vosges), Halllaxt, Fl. pop.

potte dé chwau, f., (lèvre de cheval), Raon aux Bois (Vosges), Idem.

p'houtottes de chevaux, f. pl. Padoux (Vosges), IdeM.

paitte dé chwau, f., (patle de cheval), Uriméni l(Vosges), Idem.

pourpie bouda, m., Meuse, Labourasse, Gloss. du pat. de la Meuse.

mor-cheval $\left({ }^{2}\right)$, Anjou, MÉnière, Gloss, angevin, 1880.

peta-saouma, f., Basses-Alpes, Annales des Basses-Alpes, II, p. 13.

peto-saoumo, f., envir. d'Avignon, PAlun, Catal. des pl. du terr. d'Avignon.

- Forcalquier, c. par M. E. Plauchud.

piépou-rave, m., canton des Riceys (Aube), Gúsin, Statist. du canton des Riceys, 1852.

pas de chat à ognons, m., env. de Domfront (Orne), c. par M. Aug. ChEVALIER.

(1) Selon les paysans cette plante fait naitre des douves ou fascioles dans le foie des moutons qui en mangent (DEs ÉTANGS). - De ce passage il résulte que l'étymologie donnée par LitTRÉ de douve (grande et petite douve) manque de fondement. (LiTTRÉ croit, à tort qu'ici le mot douve signifie fossé).

(2) Les chevaux qui mangent de cette plante peuvent en périr. (MÉxı̀̀re). 
erba de boc, f., Aime (Savoie), comm. par M. Marjollet. rapo di santo Antonio, italien, Rogeri, Catalogo d. piante, 1677.

silión, Plaisance, Braccifortr, Flora piacentina. radicchia, sicilien, Buxca, Flor'a d'Avola, 1842-1847.

rapaingule, Abruzzes, linasore.

cortescia, Brianza (Milanais), Cimenubin.

spilli d'oro, margheritine, capo di turco, italien.

spell d'ôr, romagnol, MoRRI.

yerba velluda, espagnol, Colmeiro, Jicc. de los nombres.

patèlo, galicien, VALLAdares.

drüsu'ur: allemand, Grinn, Deutsches Wört.

sanct Antoni rüblein, Ulm et Alsace, Priezel et Jess.. Vollisn.

spinnenwur: $\mathrm{U} / \mathrm{lm}$, IDEM.

zängerkraut, Tyrol, IDEM.

rübenhahnenfusz̃, Prisse, Frischnier, Preuss. Wört.

sint Antheunis taeplien, thamand, DoDoxiseds. [A. DE C.]

sint Anthonie's raapje, holland, Oudemans. A. LE. C.

kraaiknie (= genou de corneille), kruiknie, flamand, DE Bo. [A. DE C. saint Anthouy's rape, saint Anthony's turnep (1), anglais, Pror. Popul. names of plants.

rape crowfoot, cuctioo-luds, anglais dialectal, BrItTex et Hocland, lict. paigle, Suffolli, Foввг, Tor. of East Inglia.

fair grass, Feosse, Jamesox, Etym. dict.

chwys Mair (= sueur de la vierge Marie), blodeuyn y' ymenyn (= fleur du beurre), gallois, HrGH DAvies. [H. G.]

fuile thalmhainne (= blood of earth; it exhausts the soil), gacilique écossais. Cameron. [11. G.]

solluifrer, solviser, suédois dialectal, JENxsEx-Tuscir.

ma:ieji laksztal, lithnanien. ЈАСову, 1884.

\section{2. - USAGES.}

On emploie ses feuilles comme vésicantes. Mér.ıt, llict. de mat. méd.

C'est l'herbe de laquelle trempée en sang de dragon, les gueux malheurenx se frottent les bras, jambes ou cuisses, pour se les exulcérer à fiu qu'ils puissent esmouvoir le peuple à compassion pour tirer plus grande aumosue. - La Maison rustique. (XVIe siècle).

(1) From ils tubers heing a favourite food of pigs. (Prion). - On sait que le cochon est le compaguon de Saint-Intoine. 


\section{RANTICLLS ARTENSIS LINEE - LA RENONGULE DES GHAMPS ( 1 .}

ranunculus echinatus, ranunculus arcorum, ranumulus satirus, ranunculus segetalis, anc. nomenclature, Bachis. Pinax theatri botanici, 1671.

renoncule des champs, chausse-trape, chausse-trape des blés, français. turlugeotte, f., teurlugeotte, f., cnv. de Semur (Còte-d'Or), c. par .I. H. MARLOT.

bec de corlin, m., arrond. de Bayeux, Fr. Plideft. [Ed. Edu.

hachinet, m., Saint-Pol Pas-de-Cal.), c. par M. Ed. Edyoxt.

bassin des champs.m. lirosses, f. pl., (les fruits), Jouy-en-Pithiveray (Loiret, c. par .I. J. PoQLet.

aourifol, m., aouriflon, m., aoubrifon, m., aoubrifel, m.. Areyron,

VAsssier, lict. du pat. de l'.leegron.

aoubrifro, aourifo, Aveyrou, Revue de l'A t'eyron et du Lot.

yarafol, m., olvo, f., arr. de Brive (Corrèze), comm. par II. G. de LépIXaY. gaffarou (), m., Sud-0uest du Lauguedoc, Dubotz.

gafarot, m., garafot, m., regognou, m., horboris, 11.. embrooul, Aveyron, VAssier, Dict. du pat.

escaheret, m, Luchon (Pyrenées centrales), Jul. SAcaze.

gahoman, (= qui prend la main), Lot-et-Garonne, e. par M. l'abbé

L. DARDY.

cmbrouille, f., engrogne. f., Centre de la France, Jalbert, Gloss. du centre.

gratâ, (masculin phriel), Denx-Sevres, cmum. par M. B. Soccuk. - Chareute-Inf., comm. par E. II. Lenaniè.

grètias, m. pl., Chattillonnais Cüte-d’or, c. par M. H. JARLot.

graterons (3), m. pl., Aube, DEs ÉrAxgs, Noms pop. des pl. - Haute-

Marue, comm. par M. L. Aunriot. - Côte-d Or, c. par I. H. MARLot. grateret, m., patte d'oie, f., Haute-Marne, comm. par A. DAGrix.

(1) Cette plante porte souvent les nèmes noms que les tenoncules précédentes. Voyez les articles ci-dessus.

(2) De gaffa - accrocher. Duboul. - Ce nom et quelques-uns de ceux qui suivent, sont le plus ordinairernent lunnés aux fruits qui sont verruqueux et munis de crochets.

(3) Du verbe gratter, à cause des épines qui garnissent le fruit. 
gratons, m. pl., graterais, m. pl., petites brosses, f. pl., envir. de Semur, (Còte-d'Or), c. par M. H. MarLot.

piquerau, m., Anjou, Desvaux. - Bas-Gâtinais (Poitou), Rev. de phil. franc., 1863, p. 120.

piquwas, m., Ile d'Elle (Vendée), Rer. de phil. frans., 1889, p. 108.

macriè, m., pico, m., pièco, m., Anjou, Desvaux.

brûlante, f., Normandie, JoRET, Flore pop.

rapoule, Allier, comm. par $\mathbf{M}$. C. Bourgougnon.

patte d'oie, f., Orne, LetacQ.

pé de grolle, m., Deux-Sèrres, comm. par M. B. SorcuÉ.

pas dlagache, m. Samer (Pas-de-Cal.), c. par M. B. DE Kerhervé.

ranonculo galet, f., Var, Hankr. - Porquerolles, OLlivier.

galet, m., Var, Amic, Considérations topographiques sur Brignoles. 1837. aiwi, Spa, LezAack, Dict. des noms wallon des pl.

bramefouam (1), Issoire (Puy-de-Dòme), comm. par M. J. BAREIRE

chinot, m., (c.-à-d. petit chien', Médonville (Vosges), Haillant, Fl. pop. goussés, m. pl., (= chiens) (les fruits) $\left({ }^{2}\right)$, Villeneuve de Lauraguais,

c. par M. P. FAgot.

quoue dé renâ, f., Romont (Vosges), Halllaxt.

ranuncolo dei campi, signorine salvatiche, ital., TARgion, Dict. bot.

presora, Vérone, Pollini, Flora veron.

erba corona (3), Trévise, SAccardo, Flora trevig.

jèrbe nère, Frioul, PIRona, Vocab. friulano.

sackilieben, striegel, Wurtemberg, Pritzel et Jessen, Volksn. d. P/l.

düwelshus, Goettingue, IDEM.

knüli, chinde, chnünä, Grisons, Durhem.

pimpoele, pinpoele, flam., DE B0. [A. DE C.]

polpit, tnijtnit, flamand des envir. d'Alost, c. par M. A. DE Cock.

jackweed, hungerweed, goldweed, hedgehog, crow's claw's, gye, urchin crowfoot, hellweed, devil's claws, devil's coachwheel, devil on both sides, anglais dialectal, Britten et Holl.

hard iron, Centre de l'Angleterre, Manshall, The rural economy of the midland countries, in-8, 1790.

(1) C'est-à-dire qui appelle la faim, parce que cette plante fait le plus grand tort aux récoltes.

(2) Les fruits sont ainsi appelés parce que leurs piquants les font adhérer aux talons nus des paysans et sy attachent comme des chiens qui mordent. [P. FAgor.]

(3) Erba corona, denominazione derivata della disposizione a corona dei suoi frutti (carpelli). (SACCARD0). 
devil's curry-comb, worry-wheat, Jack-o'-two-sides, Shropshire, Jackson, Shropsh. word-book.

bygsädljeger (= fleurs des semailles d'orge), danois dialectal, c. par

M. H.-F. FeilberG.

babin $\approx u b$ (= dent de vieille femme), serbo-croate, ŠulEK.

\title{
RANCNGLLLS PHILONOTIS. (EHRHART, 1788).
}

ranunculus sardous, (1) nomencl. de Crantz, 1769.

erba di Santo Martino, Calabre. Pasquale et Avellino, Flora medica.

\section{R.NTNGLLISS SGELERATITS. (LINNÉ). - LHERBE SARDONIQUE.}

\author{
1. - NoVs de cette plante.
}

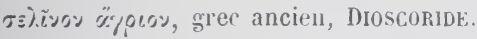

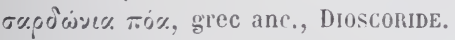

apiastrum, latin, Pline, IIst. nat. (Cette identification a été établie par

Eugène Fournien dans ses Essais d'archéologie botanique, 1878).

herba sardonia, latin du III siècle après J.-C., Soliv, Polyhistor, 4.

sardinia, latin du IVe siècle après J.-C., TiIEodorus Prisciants, cité par

MEIEn, Geschichte der Botanik, II, 298.

apium rusticum, apium risus, herba scelerata, apiastellum, dentaria, au-

ricomum, latin (Ve ou VIe s. apr. J.-C.), Apuleies, De medicaminibus. herba scelerata, latin du IXe s. apr. J.-C., Pseudo-Apclée, édité par

F. Lenormaxt, Note sur une vignette (dans Bull. de la soc. bot., 1855, II, 315-320). - lat. du moyen-âge, Howat, Ilphita.

scelerata, latin du moyen-âğe, Diefenbach, Gloss. lat. germ. med. lat. botracion, brutacea, brutacia, brutaceoci, herba mirtilana, murtillana,

ferula, smurnon, aquileia, aquilaria, latin du moyen-âsge, MowAT, Alphita, medicobot. gloss., 0xford, 1887.

ranunculus strumea, scelerata Apuleii, herba sardoa, ranunculus sardo-

(1) C'est-à-dire : renoncule de Sardaigne; voir, sur la synonymie savante de cette plante, Auguste Gras, Synonymie d'une esp. de ranunculus dans: Bull. de la soc. bot. 1862, p. $324-336$. 
nicus, apium aquaticum, ranumsulus aquaticus, ranunculus palustris, nomenclature du moyen-àge et de la Renaissance, Baums, Pinax theatri botanici, 1671.

apium ranimum, latin du XVIe s., Anoorso, le renenis, 1562.

liebiliegi, latin du XYIes. (l'apris l'arabe d'Avireune), IDEM.

herbe sardonique, f., français anc. et moderne.

ache de Sarlaigne, anc. frans., Proprietez des simples, 1569, 1. 936.

ache de ris, ane. frans., Grand herbier en fransoys (ver's 1520), fo VI, verso. ache de risée, $\mathrm{f}$, anc. frang., Maison rustique (XVIe s.'

ache riente, f. anc. frans., J. Crévix, Deux lives des venins, 1568, 1. 987. apiostra, f, ane provengal, Rarocari, Lexique roman.

herbe de feu, f., Camembert (Normindie), Joret, Fl. pop.

erlo de las bourugos, f., (= herbe des verrues), Lot-et-Gar., c. par II. l'ahbé

D.IRDY.

grenoullheta, f., Suisse romande, Bridel, Gloss. du pat.

erbo de yrapaou, f., Sud-0nest du Languedoc, Duboul.

appio riso, erba sardoa, erba sardonia, sardonia, ranuncolo di padule.

ranuncolo palustre, italien.

apiu risu, sicilien, Mortularo.

erba giacca, milanais, BA.fi, loc. mil.

sédol, larme, Malaspina. Voc. parm.

perlenebol, Brescia, Melchioni, Vor. bresc.

apiu, Sicile, Macaluso, Voc. sicil., 1875.

sardonia, yerba sardonia, malvala, riso sardonico, apio sardonico, riso

sardonio, espagnol.

yata rabiosa, espagnol, CoLneiro, Dicc. de los nombres.

erloa sardoa, galicien, Cureiro, Dicc. yallego.

putalou dos valles, portugais, Brotero, Flora lusitanica.

brandliraut, brennewu:, moyen haut allemud, Diefexbacil, Gloss.

froscheppich, froschpfeffer, allemand, Nexsich, Catholicon d. Natury.

lineclientinie, Saxe, Pritzel et Jessex, Volhsnamen.

schnifchen, Prusse, Friscimier, Preuss. Wörterb.

gichtliruud, bords de la Weser, Focke, Vollisth. Pflanzennamen, etc., 1870.

dïwelsbitt, Jord de l'Allemagne, Focke, Pflanzennamen; Strackerjan,

Aberyl. d. Herw. Oldenbury, 1867.

blartrekkende hoterhloem, jeukikruid, holland., OLDEuns. [1. DE C.] chuthung (1), chuvurt, anglo-saxon, Cockarse, Leechiloms elc. of early England, 1886. 
ache, anglais dialectal, Britten, Dict.

crafang yr eryr (= griffes de l'aigle), gallois, J. Davies, 1632. H. G.]

torachas biadhain, gaélique écossais et irlandais, CaueroN. H. G.]

tiggarranunkel, suédois dialectal, Jennsex-Tusch, Nordislie planten.

kjelsólöje, norvégien dial., IDEM.

tiggerranunkel, sliarp hanefod, pugepeber, vanimorke, danois dialect. IDEM.

ljutič, ljutil, lisičnjak (= plante des renards), samojid, svodnica (= entremetteuse), pivćja noga (= pied de coq), rranja noga (= pied de corbeau), serbo-croate, ŠLEK.

jaskier jadowity, polonais, LINDE, Slown. jez. polsk.

tzar-zille (= la plante-roi), petit russien, c. par II. Th. Volkov.

dederwynês żoles. lithuanien, JАСовт, Litauische Pflanzennamen.

csomor, csomorilia, magyar, Mıкцоsich, Slav. Elem. im mag., p. 68.

zaghlyl, arabe égyptien, DELILE, Floræ ægyptiacæ illustratio (dans Description de l'Egypte, 1824).

\section{2. - ISAGES}

Cette plante passait autrefois pour causer des empoisonnements qui provoquaient un rire particulies, le rire sarlonique.

Voici ce qu'en dit SoLin (Polyhistor, 4) :

Herba sardonia rictu diducit ora, ut qui mortem appetunt, facie ridentium intereant.

Sur le rire sardonique, voyez :

Matthioles, Comm. sur les six livres de Dioscoride, trad. par ANr. DU Pinet, Lyon, 1655 , p. 248.

Moris, Flora sardoa, 1837, in-8, I, 37-38. Ce botaniste fait de nombreux renvois aux auteurs ayant traité la question et cite les passages des anciens qui s'y rapportent.

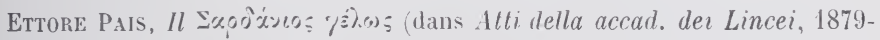
1880, Ser. terza, Memorie, etc., p. 54 à p. 74.)

Ludwig Merchlin, Die Talos-Sage und das sardonische Lachen... [Le mythe de Talos et le rire sardonique. Contribution à l'histoire du nythe et de l'art grec. Extrait des Hémoires des savants étrangers, tome VII, avec 2 pl. Saint-Pétersbourg, 1851, gr. in-4.]

Auguste Gras, Synonymie d'une espèce de ranunculus dans Bull. de la soc. botanique, 1862, t. IX, p. 324-336.) L'auteur démontre que l'on sait peu de choses sur la plante qui passait pour causer le rire sardonique, qu'en tout 
cas elle n'a rien à faire avec la Sardaigne et que quelques auteurs ont eu tort de l'identifier arec les herbae sardoae de Virgile et d'Horace. Par ce terme générique on désignait les plantes amères de la Sardaigne qui étaient cause de la renommée d'amertume du miel de cette île.

\section{RANUNGLLUS FLA.MILLA. (LINNÉ). - LA}

\section{PETITE DOUVE.}

NOMS.

ranunculus longifolius palustris minor, flammula ranunculus, ranunculus flammeus minor, anc. nomenclature, Bavhis, Pinax theatri botanict, 1671.

sayette, f., Mons, Semertier.

sansaie, f., wallon, SEMERTIER.

douve ( $\left.{ }^{1}\right)$, f., petite douve, f., flammette, f., petite flamme, français.

deure, f., Mayenne, comm. par un botaniste de la Mayenne.

dourre, drouve, droue, mort aux moutons, Normandie, Joret.

dôrve, f., Montbéliard, Contejean, Gloss. du pat.

endario, f., Gard, comm. par M. P. Fesquet.

erbo dei douro, f, Forcalquier, c. M. E. Pladchud.

endervo, f., flamaduro, f., Basses-Alpes, c. par M. E. Plauchud.

olvo, $\left({ }^{2}\right)$, f., Corrèze, G. de LÉpinay, Noms des pl.

langue de brebis, f., Bayeux (Calv.), Fr. PluQuet. [Ed. Edr.]

tiregotte, f., Vallée de Cleurie (Vosges), Thiriat, La vallée de Cleurie.

herbe à la tire-youtte, f., Centre, Jaubert, BOREau. - Haute-Marne,

comm. par M. L. Aubriot. - Côte-d’Or, c. par M. H. Marlot.

tarbero, f., talbero, f., arrond. de Saint-Pons (Hérault), BARThĖs.

erlo d'éntée, f., (= herbe de langueur), Lot-et-Garonne, c. par Il. l'abbé

L. DARDY.

yèp' de sansowe, f., sansowe f. (= sangsue), wallon, Feller.

yerba de la flamula, espagnol, CoLmeiro, Dicc. de los nombres.

speerkraut, egelkraut, gichthraut, schwefelkraut, grensing, allemand,

Nemnich, Catholicon.

(1) Le mot français douve est une simplification pour herbe à la douve, c.-á-d. herbe qui cause, aux moutons qui en mangent, la maladie appelée douve. - LiTtré, dans son dictionnaire, interprète donc à tort.douve par fossé; (herbe des douves = herbe des fossés, parce qu'elle croît dans lieux liumides).

(2) Olv'o est aussi le nom de la maladie appelée douve des moutons (G. de LEPINAY). 
kleines sperrkraut, brennender hahnenfuss, allemand.

eghel-koolen (= chou des hérissons), flam., Dodosaeus. [A. DE C.]

kleine egelkolen, egelgras, hollandais, Oudemans. [A. DE C..

yellow crane, anglais dialectal, BritTex, Dict.

baneworl (= racine poison) ( ${ }^{1}$ ), anglais, Prior, Pop. $n$. of plants.

blaen y groayw (= pointe de la lance), fflamboeth, fflainmog i= l'enllam-

mée), gallois, Meddygon myddfai. [H. G.]

poethfflam (= flamme brûlante), gallois, J. DAvies, 1632. [H. G.]

lassair lena, irlandais, THRELKeld. [H. G.]

lasair leana (lasair $=$ flame; leana $=$ swamp, spear), glas-leun (glas $=$

green ; leun = a swamp), graélique écossais, Caverox. H. G.

ältgräs, iktegräs, lijülleljunk, briindört, vä̈rkört, suéduis dialectal

JENSSEN-Tusch, Nord.

renselsgras, myrtrauska, norvégien dialect., IDEM.

froepeber, pugepeber, gigturt, jeglblomme, danois dialectal, InEN.

plamenka (= plante de flamme), plamuša, ̇̇ujka, suncena iskrica

(= étincelle du soleil) petehova noga (= pied de coq), porodni

żabjek (= plante des crapauds), serbo-croate, Šulek, Jugosl. im. bilja.

gorcyca (= amère), wende, Sciulexburg, Wendisches Vollisthum, 1882. liutik, russe, Falk, Beytr. *. top. Kenntn. d. russ. Reichs., 1786.

mazieji luksztal, lithuanien, JАСову, Litauische pflanzenn.

gaila pehdas, gaito kahjas, letton, ULmaxi, Leltisches Wörterbuch.

maanpakiana, finnois, Fellara , Ind.pl. fennicarum, 1835.

\section{RANUNGULLS LINGLA. (LINNÉ). - LA GRANDE DOUVE.}

ranunculus longifolius palustris major, ranunculus lanceolatus major, ranurculus flammeus major, ranunculus insectus, lingua Plinii, ancienne nomenclature, Bauнin, Pinax theatri botanici, 1671.

grande douve, f., douve, f., français.

erbo dei douvo, Forcalquier, c. par M. E. Pladchud.

lagagno, f., sud-ouest du Languedoc, Duboul.

bugallon, galicien, Cuveiro, Dicc. gall.

egel-koolen, flamand, Roucel, Flore du nord de la France, 1803.

sumpfhahnenfuss, zungenblätterriger ranunkel, allemand.

(1) From its being supposerl to bane sheep; Salmon tells us that it does by ulcerating the entrails (PluOR). 


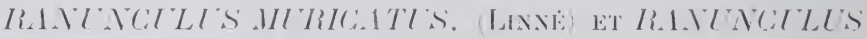 GRAECLS. GRISABACH).}

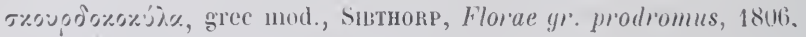
ranonculo rubisso, Var, Haxrs, Calalogue.

ranunculu, sonnu grassu, Sardaigne du Nord, Moris, Floru saidoa. liosberbir, arabe algérien, Dovernier, Les Touaregs du nord, 1864.

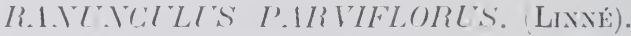

picot, in., Anjou, Dessalis, filore de l'Anjou.

herbe grasse, f., Charente-Infér., comm. par M. E. LEMariE.

\section{RANT NEILIS THOR.A. LANÉ). - ET R.ANGNCLLLS I'SELII)O. THOIR. (HosT).}

bassenel, m., cabarel, m.. Suisse romande, Vicat, Plantes rénéneuses. alpen giftranunliel, allemand, Rosexthat, Syn. pl. diaple.

otrornica (= l'empoisonneuse, serbo-croate. Šulek, Jugosl. im. bilja.

\section{RANTNGILLS BILLATLS. (LINAE,}

bolon de Portugal, espagnol, Colueiro, Diccion.

flor de Sun Diego, Cordoue, Borssier, loyage botanique dans le midi de lEspagne, 1839-18.15.

frol de San Diego, erba belida. galicien, Valladares.

montúa de outono, Brotero, Flora lusit.

scheqiq en na'mân, arabe algérien, Duraxuo. Calendrier de Flore algér.

saghlil, arabe des environs d'Alger, Jourds., Flore murale du tombeau de la chrélienne, 1867.

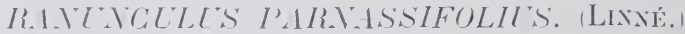

e?lua del mal grí, Vall de Nuria (Catalogne), VAYreda.

\section{RATVGILLS ALRKCOMLS. LINEE).}

peneuraide, gallois, Hugh DAries. [H. G.]

follasyain, gaélique ícossais, CAMErox. H. G.

folescain (= a ladjole), irlandais, Camerox. [H. C.] 
R.IVTVGTLS GLACIALIS. LINNÉ.

calline, f., caicaline, f., sud-est de la France, LAMarck et Caxdolle.

\section{RA.TYGILISS BREVIFOLIIS. (TEXORE).}

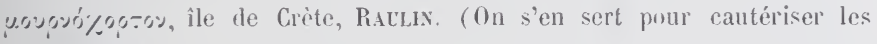
verrues.

\section{FICARIA R.ANTXCILOIDES. (ROTH). - LA FIGMIRE.}

\section{NOMS DE CETTE Playte :}

Qu.yir, grec ancien do Théophraste, selon Sprexgel (dans son ádil. de

Throplur.) (Sprengel ne donne cette identification qu'arec doute.

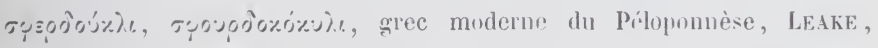

Travels in the Morea, 1830.

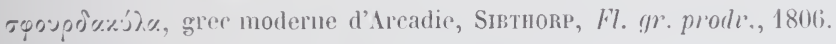

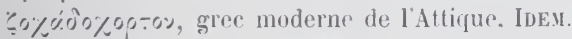

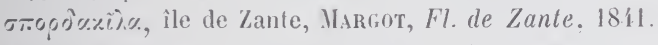

chelidonia minor, chelidonium minus, ficaria minor, s"rophularia minor,

testiculus sucerlotis, hemorrhoidum herba, malacocissus minor,

anc. nomenclature, B.suns, Pinax theati hotan.. 1671.

testiculus sacerdotalis, testiculus prespiteri, latiı dı Moyen-Age d’après

d'anciennes gloses, Schueller, Bay. Wört. I, 1054.

mentula, mentula episcopi, pes corvi, pes corvinus, apium emoíoila'um,

latin du Moyen-Age, Mowat.

appium emoroydarum, botracion, staticere, iran, nomenclature du

XVe siècle, J. CAmes, L'opera salernitana, ete., p. 36.

ranunculus ficaria, nomenclature de Linsé.

ficaire, f., renoncule ficaire, $\mathrm{f}$, herbe an fic, f., ficaire renoncule, f., petite chélidoine, f., herbe aux heimorrhoides, f., petite éclaire, f., iclairette, f., petite scrophulaive, f., français.

petite esclaire, f., bassinet, ancien français.

petite claire, f.. éclairotte, f., Haute-Marne, conm. par II. A. Dagun.

clatiotte, f., Jony en Pithiverais (Loiret), e. par M. J. Po@Let.

clarégeassa, f., Luchon (Pyrénées centrales, Julien Sacaze, Fl. de Luchon. boutaillets, m. pl., Vallorbes (Suisse romande), VallotToN-AUbert. godet, m., godat, m., godinot. m., Aube, Des Étaxgs, Noms pop. des pl., 1814.

clair bassin, m., Аијоu, Desratx, flore de l'Anjou.

cllar bassin, m., Deux-Sìres, comm. par MI. B. SolcuÉ. 
bouton d'or', m., Charente-Inférieure, comm. par M. E. Lemarié. - Environs de La Rochelle, Annales de la Soc. d'agric. de la Rochelle, 1854.

- Normandic, JoRET.

boutoun d'oro, m., Apt, Colignon, Fl. d'Apt.

bouloun de gat, m., Bouches-du-Rhòne, Villeneuve, Statist. des B.-du-Rh.

- Basses-Alpes, Annales des Basses-Alpes, II, 17.

poumpoun d'or, m., Hérault, LoRet, Fl. de Montpellier; Barthès, Gloss. pounpoun d'or', m., Hérault, Albert FanRe, Hist. de Saint-Georges d'Orques, 1882.

louis d'or, m., Mayenne, comm. par un botaniste de la Mayenne.

gannet, m., jaunet, m., jaunette, f., Normandie, JoREt, fl. pop.

ganne d'œu, m., tite éclaire, f., Saint-Pol (Pas-de-Calais), c. par M. Ed.

EDMONT.

jauneau, m., Anjou, Desvaux, Fl. de l'Anjou.

jaounet, m., La Hague (Manche), Fleurr, Ess. s. le pat. norm.

pissenllet, m., Guernesey, Métrivier, Dict. franco-norm.

pissenlliet rond, m., Bessin, JoRET, Fl. pop.

pissenlit rond, m., Anjou, Desvaux, Flore de l'Anjou.

pissenlit doux, m., Anjou, Desraux. - Mayenne, comm. par un botaniste

de la Mayenue.

pissolièch, m., Aveyron, Varssier, Dict. du pat.

caillonade, f., Charente-Inférieure, Annales de la Soc. d'agricult. de la

Rochelle, 1854, no 18, p. 90.

girounée, f., Charente-Infér., comm. par M. E. Levarié.

giron, m., Saintonge, Brunaud, Liste des pl. de Saintes, 1878.

ciro, f., sud ouest du Languedoc, Duboul.

billonée, f., Centre, Boreau, Flore du Centre. - Anjou, Desvaux, Flore. couténos, f. pl. (= couennes, peau râclée du porc, à cause de l'épaisseur

des feuilles de la plante), gascon, c. par M. l'abbé L. DARDY.

pieds d'polain (= pieds de poulain) m. pl., Ban de la Roche, H. G.

Oberlin, Descript., 1806.

pied de polain, m., Moussey (Vosges), Haillant, Flore pop. des Vosges.

pieu de polain, m., Vexaincourt et Wisembach (Vosges), IdeM.

pé de grole, m., Deux-Sèvres, c. par M. B. Souchí.

paoutoloubo, f., Saint-Germain (Lot), Soulí.

lampaouto, f., lempaouto, f., limpaouto, f,, lompaouto, f., Aveyron,

VAYssier, Dict. du pat.

étaila tzóna (= étoile jaune), f., Suisse romande, Bridel, Gloss.;

Durheim, Schw. Idiot.

coupa verruga, f. Luchon (Pyrénées centrales), Julien SACAze, Flore. rai á la figue, f., fiquetla, f., Suisse romande, Vicat, Plantes vénéneuses. 
figuetta, f., Suisse rom., BRIDEL, Gloss.

erlo dei barrugo, Forcalquier, c. par M. E. Plavchud.

ache de emorroydes, f., anc. français, J. CAuus, L'opera salernitana, p. 36 .

erba de las morenas, $\mathrm{f}$ (= herbe des hémorrhoïdes), Pyrénées-0rientales, Companyo, Hist. nat. des Pyr.-Or.

erbo de las mourenos, f, Gard, comm. par M. P. FesQuet.

glaoubanel, m., gascon, Novlet, Fl. du bassin pyiénéen. - Toulouse,

Tournon. - Tarn-et-Garonne, LAgrèze-Fossat. - Sud-ouest du

Languedoc, Duboul. - Villefranche de Lauraguais, c. par M. P. FAGoT. glaübanel, m., gascon, Noulet, Flore du bass. pyr., 1837.

claouanet, Sud-Ouest du Languedoc, Duboul.

graouxol, Lot, c. par M. J. DAYMaRd.

graoujol, Tarn, Tarn-et-Garonne, c. par II. J. DAYMard,

mire-soleil, ancien français, DecAisne, Catal. des pl. du livre d'h. d'Anne de $\mathrm{Br}$.

aourelhetas, f. pl. Basses-Alpes, Annales des Basses.1lpes, II, p. 17. aourilleto, f., Bouches-du-Rhône, Villeneuve, Statist. des B.-du-Rh. aouriheto, f., Avignon, Palun, Catal. des pl. d'Avign.

oureheto, Forcalquier, c. par M. E. Plauchud.

aoureilleto, f., aoureieto, f., Apt, Colignon, Fl. d'Apt. - Var, Hanrx,

Ciatal. des pl. - Aix, Boyer de Fonscolombe, Calendr. 1845.

ouriette, f., Arles, Laugier de Chartrouse, Nomencl. pat.

couillons de prestre, m. pl., ancien français, Duchesne, De stirpibus, 1544.

- Pinaeus, Hist. plant., 1561. - Linocier, Hist. des pl., 1584.

couille à l'evesque, f., ancien français, Cotgrave, French dictionar'y, 1650. ranounclës, canton du Vigan (Gard), Rovger, Topogr. 1819.

bienoulevie, f., Centre, Jaubert, Gloss. du Centre. - Boreau, Fl. du

Centre.

épinard des bücherons, m., Haute-Marne, comm. par M. L. Aubriot.

ail de crapia, m., Ile de Ré, comm. par M. E. Lemarié.

rondotte, f., Doubs, Beavquier, Voc. du Doubs.

rondlotte, f., Romont (Vosges), Haillant, Fl. popul.

moruites de terre, f. pl., environs de Caen, JoREt, Fl. pop. norm.

vilettes eud' bure ('), f. pl., environs de Cambrai, Boniface, Hist. du vil-

lage d'Esne, 1863.

arolle, f., Centre, Jaubert, Gloss.

(1) Dans le pays de Cambrai, le mot vilette signifie fleur en général. Par conséquent la traduction est fleurs de beurre. 
cocarle, f., Ille-et-Vilaine, Orain, Gloss.

courdoun yat, Gers, comm. par M. H. Daignestor's.

biscouloun, sud-ouest du Langruedor, Duroul.

favoscello, italien, Matтhiol, Comment. de med. mat., 1598

favosello, Bologne, Ambrosivi, Phytologia 1666.

fa'arella, badile, italien, IDEM.

coglie di prete, italien, AwgillaAm, Semplici, 1:661.

favayello, Tuscane, Carsalpivios, De plantis, 1583.

celidonia minore, chelidonia minore, ceneroynola minore, fasayello,

faugello, fau'ascello, favegello, favuccello, formes diver'ses italirmes,

TARGIONI.

favarella . piadanella, romagnol, Morri.

fla'ayello, Suse, F. Re, Flora segusina, 1881. J. C.

orie dij fra, aurie d'i fra, piémontais, G. Canssola, Flora astense, 185 .

[J. C.]

omrie l'rot.piémontais, A. BoLra, llerbarium perlemontanmm, 1837. [J. C.]. far'oscello, erba majestra, erba fara, farajola, Tessin. Zeitschrift für die

(1. Mythologie, $\mathrm{N}, 177$.

erba majestra, milanais, CIIERt BINI.

erba soralonne, milanais, BANF, Voc. milanese.

orcela, Parme, Mal.ispina, Voc. parm.

erly' e ranas, Sardaigne du Nord, Monis, Flora sarloa.

pedonchet, piémontais, Cirello, Mact. piem., 1814.

ciapparerlda, sicilien, Bisxcis, Flora d'Avola, 1842.

scrofularia menor, espagnol, J. Victon, Tesoro, 1609.

yotıssu, Baléares, Marès, Catal., 1880.

erba das almorranas, galicien, Cuveno, Dicc. gallego.

celidonia menor, portıgais, BRotero, Fl. lusit.

saloyea, calce mica ('), ierba-rindunelei-mici, untishor, salata mielului,

ronmain, BваND\%, Limba lot., 1882.

salatsé $\left({ }^{2}\right)$, roumain, Сıнас, Dict. daco-roman.

slieltsch mitsch. jarbe rindunïlie, roumain de Transylvanie, Fusz, Trivialn.

in Sieb., 1848.

graîshor, roumain moldave, Braxdza, Limb. bot.

feycliurtzenkraut, meykraut, allemand, Corbes, Botanologicon, 1533.

scorbocks lirtmt. allemant (apud Saxones), IoE.M.

(1) Dans calce mica le mot calce correspond au latin caltha, nom dine autre renonculacée.

(2) On mange les feuilles de celle plante en salade, au printemps. 
pfaffenhoetlin, fergwurtzenkraut, blaternkraut, kleyn schwalbenkraut, meyenkraut, allemand, DoDonæus, Herbarius, 1608.

pfennigsalat, rannenhödlein, biberhödlein, lämmerblume, scharbock, rapunzchen, allemand, Nемкісн, Cathol., 1793.

erdgerste, feigwarzenkraut, allemand, GRIM, Deutsches Wört.

kleines schölliraut, scharbockshraut, mäusebrod, allemand, RoSENTHAL.

Synopsis plantarum diaphor.

pfaffenhoden, bavarois, Schmeller, Bayer. Wört.

hä̈lielezalat, Transylvanie, Pritzer et Jessen, Volksn. d. Pfl.

henkelnzaloat, allem. de Transylvanie, Fusz, Trivialn.

himmelmehl, Autriche, Pritzel.

feigwurzel, Wurtemberg, IDEM.

butterblätter, Eifel, Wintgen, Veget. 1.Eif., 1865.

kreuะerlan, schmalzblattl, schmalzplötschlan, zigeunerkraut, zigeunersalat,

Carinthie, Zwanziger.

eglhraud, schiorbockwurะn, schmalzbleaml, Autriche, F. HOEFER.

schuôrbock, Luxembourg, KoLTz, Flore du Lux., 1873.

sclimernel, schmirgel, Prusse, Frischier, Preuss. Wïrt.

fettgitchen, allemand de Livonie, IIUPEL.

jägerkraut, Grisons, DurheIM, Schw. Idiot.

bettlerkraut, Oberland bernois, lvem.

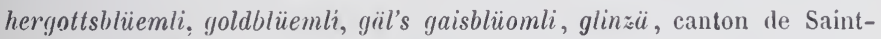
Gall, Wartuann, Saint-Gall. Volksbot., 1874.

gliserli, sternblümli, Suisse allem., Zeitsch. f. d. deutsche Iythol., IV, 177. speencruydt, kleyne gouwe, flamand, DoDoneus, Cruydtboeck, 1644.

[A. DE C.]

haane-klootjes, Arnheim, DE Gorter.

hanehlootjes (= testicules de coq), hollandais, Oudemans. [A. DE C.]

speenkruid, boterbloem, haagboterbloem, tlam. et holl. [A. DE C.]

cluvihte, anglo-saxon, Cockarne, Leechdoms, etc., 1866.

gollan, anc. anglais, BARTHoLomeus, Sinonoma, cité par Mowat.

crowefet, anc. anglais, Mowat.

small celandine, marsh pilewort, crain, foalfoot, butterplate, anglais

dialectal, Britten, Dict. of. pl.

pilewort, anglais, Prior, Pop. names.

basquicq, breton, Nomenclator, 1633. [E. E.]

basqicy, breton, GrÉgoIre. [E. E.]

baskik ( $\left.{ }^{1}\right)$, breton, LEGONIDEC. [E. E.]

(1) On trouve dans Liégard le mot $b \hat{a} \approx-k i k$ appliqué à la scrofulaire noueuse, ce qui signifierait baton de chair mais $b a z$ est féminin, tandis que baskik est masculin. [E. E.] 
sclaeril, (= petite éclaire), breton, GréGoIrE. [E. E.] sklérik, breton, LEGonidEc. [E. E.]

lousaouënn an dervoed, (= herbe aux dartres), breton, Grígolre. [E. E.] louะauen-ann-darioed, breton, LEGONIDEC. [E. E.]. butun-nevez ( = tabac nouveau ( $\left.{ }^{(}\right)$breton, TroudE. [E. E.]

slilerik, louzaouen ann darvoed, breton, LitGARD.

llygad y diniewed (= cil de l'innocent), llygad ebrill (= œil d'avril), llysiau 'r bronnau (= herbe aux seins), ffioled fraith (= violette bigarrée), mil, gallois, J. DAvies, 1632. [H. G.]

gwenith y ddaear (= blé de terre), gwenith y gôg (= blé de coucou), milfyw, melyn y gwanu'yn (= jaune du printemps), bronwys = mammillaire ). bromwst, (= maladie des seins), gallois, Hugr Davies. [H.G.]

lodledig wen (= fire dissolvent), gallois, C.Ameros. [II. G.] grain aigein, searraiche (= little bottle), gaélique écossais, CAMEroN. [II. G.] searraigh, irlandais, 0'Briex et 0'ReILly. [H. G.]

svalört, svalblomster, lierskiil, möja, flengrïs, prästepungar. suédois dialectal, Jennsen-Tusch, Nord. planten.

smörkop (= coupe de beurre), danois. [H. F. FeILberg.]

orsej jarmi, tehèque, A. Muelier, Wört. syn. Pfl., 1866.

psæonka, polonais, Šulek, Jugoslav., etc.

jaskier tredlowy, polonais, LINDE, Sl. jez. polsl.

samergiel, polonais, Frischier, Preuss. Wört.

tschistial, russe, Falk, Zur Kenutn. d. russ. R., 1786.

salata (= salade), petit russien, с. par X. Тн. VoLкоу.

bobolj. bobovnik, buboj, čeprlin, lirupnili, ledinjak, maslac, mačja mudasca, macji mud (= testicules de chat), mala rosopas, manja rosopast, strupnil, stupuik, slatica, slatousta, śuja, bradavičnek, lopatice, srbečica, nacista misel, višta misel, serbo-croate, Šulek.

tuhkuma saliles, letton, HupeL.

korradu belhar, basque. [J. V.]

kis lietslie-fü, füge-levelü-fü, yalambhegy, ı'ajumogyoro, golva-ronto-fü, szuly-fü, magyar, Kolbani.

sálata szirontak (c.-̀̀-d. salade renoncule), magyar, CiнAc.

aranyal-versengö, magyar.

tavaszi salàta, magyar, Fusz, Trivialn.

maan palikana, finnois, Felluax, Ind. pl. fenn.

kanna kooljad, sydamme rohluud, esthonien, IDEM.

(1) Les anciens prétendaient que le suc de cette plante purgeait le cerveau. 
marmiran zeghîr, warâq mazqoûl, baqlet et kouthatheyf (c.-à-d. légume des hirondelles), arabe syrien, BERGGREN., Guide francais-arabe, 1844.

\section{2. - DiCTONS, USAGES, CROYANCES :}

Le graoubanel - couflo lou troupel. (La ficaire fait gonfler le troupeau, c'est-à-dire les moutons.) Villefranche de Lauraguais (Haute-Garonne), c. par M. P. FAgOT.

Die Wurzel enthält Stärkemehl und ist gekocht 'essbar; die Blumenknospen, in Essig eingelegt, geben ein Surrogat der Kappern, während die schwach salzigen Blätter in manchen Gegenden als Gemüse oder Salat gegessen, besonders häufig aber zu Kräıtersuppen verwendet werden. Die an den Wurzeln, sowie in den Blattstielwinkeln sich befindenden kleinen knöllchen werden in bergigen Gegenden durch starken Regen häufig in grosser Menge zusammengeschwemmt und veranlassen so die Sage vom Getreideregen; sie werden als Himmels-Gerste oder Himmels-Mlanna von Armen gesammelt und gespeist.

RosexthaL, Synopsis plantarum diaphoricarum.

« Entre autres noms, la ficaire porte celui de graushor, les petites bulbes lavées par la pluie se répandant quelquefois sur la terre comme des graines tle blé, ce qui a même donné lieu ì la croyance populaire d'une pluie de blé. »

Roumanie, CuHac, Dict. daco-roman.

«Die Knöllchen dieser Pflanze gaben gegen das Ende der VierzigerJahre Veranlassung zur Sage vom Kartoffelregen. Sie wurdelı iiberall, selbst auf Dächern, wohin sie ausgetrockuet durch den Wind leicht getragen werden könnten, in Menge gesammelt und nachher in der Hoffnung gesteckt, gesunde Kartoffeln daraus ziehen zu können. 》

\section{Canton de Saint-Gall, WartmanN, Volksbotanik, 1874.}

D'après le principe de la signatıre, les médecins du moyen âge attribuaient à cette herbe la propriété de guérir les hémorrhoïdes, en raison de l'analogie de forme de ses racines globuleuses avec les tumeurs hémorrhoïdales. Cette croyance a persisté en Flandre : une décoction de la ficaire (racines comprises) et de la réglisse y est encore employée pour guérir les hémorrhoïdes. (Hémorrhoïdes se dit en flamand speen, de là le nom flam. de la plante speenkruid.)

A. DE Соск, Volksgeneeskunde in Flaanderen, 1891.

\section{FICIARIA CALTH/EFOLIA. (REIGHeNBaGh).}

aoureilleto grosso, f., Var, HANRY, Cat.

thibiouth, kabyle, Hanoteau, La Kabylie, 1872. 


\section{CERATOCEPHALLS ORTHOCIERAS. (DE CANDOLLE).}

plosnicariu, cornishor, roumain, BRANDzA, Limloa bot.

\section{MYOSLRUS MINT.MUS. (LINNÉ).}

queue lle souris, f., français, Dodon.eus, Itcrbarius, 1608.

cono de rato, Forcalquier, c. par M. E. PLAcchud.

mioussuro, f., Var, A»ıc, Cons. sur Brignolles.

cola lle raton, espagnol, Colmeiro, Dicc.

rabo de rato, galicien, CuvEıno, Dice. gall.

tausentiorn. allemand, Dodox.ecs, Ilerbarius, 1608.

maïseschwan:, müsenstiel, Suisse all., Pritz, et Jess., Vollisn. d. Pfl.

herrenzippel, Silésie, IDE.M.

muysen steertlens, hollandais, DoDox.eus, Ilerb.. 1608.

muizestaart, flamand et hollandais. [A. DE C.]

bloodstrange, mouse-tail, anglais, BritTEx et HoLL.. Dict.

cynffon lly!joden (= queue de souris), gallois, Hugh Davies. [H. G.]

lost rac'll, lreton de Vannes, Maréchar.

lôst râz, breton, LiĖGand.

musrumpa, mustippa, suédois dialect., Jexisex-Tusch, Norl. planten. musehale, danois dialect., IDEM.

misji rep, serbo-croate, ŠLtek, Jug. im. bilja.

myszy oyon, polonais, Linde, Sl. jez. polsl.

myschein lihiost (= queue de souris), russe, FaLk, Zur Kenntn.

\section{VIGELLA ARVENSIS. (LINNÉ) ET NIGELLA I)AMAS- CENA ('). (LINNÉ). - LA NIELLE.}

\section{1. - Nous :}

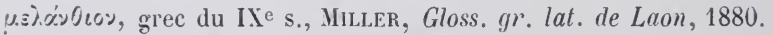

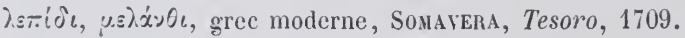

T.000\% \% \%0:0\%, grec moderne, SibTh0RP, Fl. gr. prod.

ux.00\%ó\%o, grec moderne de l'Attique, IDEm.

nigella sylvestris, melanthium sylvestre, cuminum sylvestre, nigella coruuta, melauthium damascenum (= nigella damascena. L.), ancienne nomenclature, Baunis, Pinax theatri botanici, 1671.

(1) L'espèce appelée par les botanistes Nigella damascena appartient plus particulièrement au midi de l'Europe. Elle est semblable à la Vigella arvensis, mais a des fleurs plus grandes. Le vulgaire les confond habituellement toutes deux sous les mêmes noms, qu'il applique aussi parfois à la Nigella sativa. 
gith, git, anc. nomenclat.

nigella mala, anc. nomencl., ARDorNo, De venenis, 1562.

natea, (la graine-de la nigella), latin du moven âge, Germania, 1888, p. 307.

neyalla, f., niella, f., gniella, f., Suisse romande, Bridel, Glossaire.

niello, f., environs d'Aix en Prov., Garidel.-Bouches-du-Rhône, Villexecre,

Statist. - Apt, Colignox, Fl. - Forcalquier (Basses-Alpes), c. par

M. E. Placchld.

aniela, f., canton du Vigan (Gard), Bocger. - Gard, Primes d'honneur, 1878, p. 158.

anielo, f., Card, comm. par M. P. Feseuf.T.

anièro, f., brerbo dé cupuchin, f., ouest du Lot-et-Garonne, c. par II. l'abbé

L. DABUY.

nielle, f., ('), français ancien et moderne.

nielle des blés $\mathrm{f}$., nielle des champs, $\mathrm{f}$., nielle sauvage, $\mathrm{f}$, nigelle bleue, $\mathrm{f}$,

herbe à l'uraignee, f., toute épice, f., français.

neelle, $\mathrm{f}$., ancien français, GODEFroId.

noelle, fr., anc. fr. (XIIe siècle), Bibl. de l'Ecole des Chartes, 1869, p. 331.

noiele, f., noyelle, f., anc. frans., J. CAmcs, L'opera salernitana. - Meuse, Cordier, locab., 1833.

nueule, f., (ueu se prononce d'une seule émission de voix, " très long et eu très bref, canton de Périers (Manche), rec. pers.

niole, f., gnole, f., Nièvre, comm. par M. A. Milliex.

noêlle, f., naiêle, f., Vosges, Haillant, Fl. Pop.

naille, f., Plancher-les-Jines (Haute-Saône), Pollet, Vocab., 1878.

néla, f., Tarentaise, P0хт, Patois de la Tar., $187^{2}$.

nele, f., nelle, f., nèle, f., normand, - picard, - berrichon.

léne $\left({ }^{2}\right)$, f., Yonne. - Aube. - Loiret.

poevrette, f., poivrete, f., poirrette, f. anc. franc., Dcchesse, De stirp.,

1541 ; Philiatre, Tresor des remedes, 1555 ; PIx.ers, 1561.

poirrette, f., poirrette commune, f., français.

pebreto, f., Toulouse, Tockxox, Fl. de Toul. - Lauraguais, c. par M. P.

Fagot. - Avignon, Palux, Catal. - Forcalquicr, c. par M. E. PlauCHUD.

(1) Ce mot de nielle prète à la confusion, car le vulgaire lapplique souvent encore à d'autres plantes, à l'agrostemma githago, au Lychnis githago et au Charbon des blés, espèce de moisissure.

(2) Dans l'arrondissement on appelle éléner laction qui consiste à retirer, avant de battre les gerbées, l'herbe la plus apparente, surtout la nielle afin davoir du grain plus net.

C. E. Royer, Catich. des cult. p. l'arr. de .Montargis, 1839. 
pebrino, Toulouse, Tournon, $\mathrm{Fl}$. de Toulouse. - Lauraguais, comm. par I. P. FAGot.

especis, f. pl., provenç. mod., AzAïs, Dict. des idiomes.

barbudo, f., Gard, comm. par M. P. Fes@Uet.

barbuo, f., Forcalquier (Basses-Alpes), c. par M. E. PLAcCHud.

nielo barbudo, f., Var, Haxry, Cataloyue.

barbute, f., anc. français, Honnkens, Rec. de dictionnaires, 1599.

barbue, f., anc. français, Duchesse, De Stirpibus, 1544 ; Pindeus, Hist.

pl., 1561; - franc. moderne.

burbiche, f., barbe de capucin, f., français, Rozler, 1793.

barbeau, m., cheveux de Vénus, m. pl., français, Lamarck et Candolle. barbichoun, m., Luchon (Pyrénées-Centrales), Julien SAcaze.

patle d'araignée, f., Allier. - Loiret. - Charente-Inférieure. - Pas-deCalais.

pètte de filère, f., (= patte d'araignée), Vosges, Halluaxt.

araignée, f., Centre de la France. - Haute-Marne. - Nord. - Normandie.

Doubs. - Loiret.

aréniẻe, f., arinièe, f., arégnie, f., Valenciennes, HÉcant.

arignie, f., environs de Saint-Pol (Pas-de-Calais), com. par M. Ev. Edmont. arogne, f., orègne, f., wallon, Feller.

irogné, f., Lot, Puel.

tataragnos, f. pl., (= araignées), sud-ouest du Languedoc, Duboul.

laryagno, f., Gers, c. par M. H. DAignestous.

marmite, f., Doubs, Beauquier, l゙oc. du Doubs, 1881.

armita, f., Pyrénées-Orient., Companyo, Hist. nat.

religieuse, f. Centre, Jaubert, Gloss.

tonnerre, m., roue de Sainte-Catherine, f., Aube, Des Étangs, Noms des pl. dans l'Aube, 1814.

jolet (= petit coq), m., Vexaincourt (Vosges), Haillaxt.

marie cancaille, f. Ham (Picardie), Corblet, Gloss. Picard.

micancaille, f., Noyon (Picardie), IdEM.

froumatche, Liége, c. par M. 0. CoLson.

boutet, m., Gers, Dralet, Plan de Topograph. du Gers.

oil de chat, m., Aube, Des Étangs, Noms des pl. - Haute-Marne, comm.

par M. A. Daguin. - Charente-Inf,, c. par M. E. LemariÉ.

eu d'chet, m., Meuse, Laboliasse, Glossarre.

sil de bouc, m., Pamproux (Deux-Sèvres), comm. par M. B. Souché.

ail de Christ, m., Anjou, Desvaux, Flor'e de l'Anj.

nigella, git, gittone, gitterone, gittajone, melantio, nigellastro, melantio

falso, ruosola, italien, Dodonaeus, Herbarius, 1608.

aniyella, damigelle, erba bozzolina, geppi, hysopiro, luigi, melantio sal- 
vatico, scapigliate, scarmigliate, scompigli, streghe, scompigliate (1),

fanciullace, formes dialectales italiennes, TARgioni, Diz. bot.

cominella, fanciullaccia, ital., MorRI.

agnela, piémontais, ZaLLI, Diz. piemontese, 1830.

barba de fra, Parme, Malaspina, locab, parmigiano.

panza de munega, Trévise, Saccardo, Flora treviy.

monaghela, Côme, Montr.

ragu, m., romagnol, Morri. - Frioul, PiroNa. - Brescia, Zersi.

raj, Frioul, Pinona.

occi de pavon, Vérone, Pollin, Fl. veronensis.

strie, f. pl., Gènes, Casaccia, Diz. genovese, 1873.

franlaria, Natera, GatTini.

flori di passioni, Sardaigne du Sud, Moris, Fl. sard.

fiore de passione, Sarlaigne, SpAno.

nieddone, sarde logodourien, Spano.

xiuri di Dionisi, sicilien, LAGusi, Erbuario, 1742.

lnmpiunedda, lantrunli, occhin di palumun, sicilien, Bisca, Flora dei

diutorni d'Arola, 1842.

paveréla, Venise, Boerio, Diz. veneziano, 1856. - Padoue, Patriarchi.

aranyas, arañas, catalan, Marés; - Cuni ; - VAYREda.

estrella de camp, Vall de Nuria (Catalogne), Varreda.

agennz, axenus, alpivre, alipivre, neguilla, araña, arañuela, espagnol.

negrushca, roumaiı, Сінас, Dict. daco-rom.

barba boiamlui, chica r'oinicului, roumain, BrANDZA, Limba bot.

raden, ratten, rettel, schabab, anc. allem., Diefenbach, Gloss. med. latiu. raten, anc. all., Germanin, 1881, 1. 402; PIPER, Altd.Pflanzennamen, 1881. brotwurz, protwur\%, ane. allem., Diefenbach, Deutsches Wört.; P'IPER,

Alt. Pflanzennamen.

feldschwarskiminel, ackernigelle, allemand.

venushaar, damascener schwarzkümmel, französischer schwarzkümmel,

(= Nigella damascena, L.), allem., Rosenthal, Syu. plant. diaph.

ledichtblume, allemand, G. R. Bовнмев, p. 53.

greall iu der stau'u, gredl hiuter der stauden, yriill in der stauan, Basse-

Autriche, F. HeEFER.

gretel, gretel in der staudeu, Carinthie, ZıANziger.

jungfer in grïnen, Luxembourg, Koltz. - Souabe, Pritzel.

jofer aun grengen, Luxembourg, J. WEBER.

(1) Nasce nei campi con fiore celeste, contornato dalle foglie ultime finamente intagliate, l'oude il nome di Scompigliate (TARgloni.) 
jungfer im grase, jungfer im netze, allemand, GRIMm, Wörterb.

müsken in der heede, nonnennagelein, Hambourg, Pritz. ET JEss. Volksn.

braut in haren, gretchen im busche, Silésie, IDEM.

gretchen im grünen, kapuzinerkraut, allem., A. Muelder, Synonym. Nam., 1866.

jangfer äm grähnen, allem. de Transylvanie, Fusz, Trivialnamen, 1848. jumfer in't gröne, jumfer in hâren, bas-allem., Focke, Pflanzennamen. gretli im busch, brut in grïana, greatli im struss, canton de Saint-Gall,

Wartuann, Vollishotanik.

schabab, canton de Lucerne, Durhein, Schu. Idiot.

spinnmüggli, Suisse allemande, Zeitsch. $f$. d. deulsche Mythologie, IV, 177.

juffrouwen in't haar, beschaamde meisjes, flamand, ls. Teirlinck, Plantlore, I, 1892.

kobben, flamand, DE Bo, Westvl. Idioticon.

spinnetioppen, Flandre Orientale, c. par M. A. DE Cock.

duivelsbaard (= barbe du diable), flamand (Brabant), Is. Teirlinck.

juffertjes in't groen, hollandais, Van DaLE, Woordenboek, 1884.

saint-Catherine's flower, anglais, Mnshev, Dictionary, 1623; Cotgrave,

French dict., 1650.

bishop's wort, anglais, Cotgrave, Fr. Dict. 1650.

love in a mist, fennel flower, devil in the bush, kiss-me-twice-before $i$

rise, anglais, Prior, Pop. names of. pl.

jack-in-prison, oldman's beard, anglais dialect., Britten eT Holl. Dict. love entangled, love in a puzzle, Devonshire, Friend, Glossary.

lus an fhograidh (= pursued plant), gaélique d'Ecosse, Cameron. [H. G.]. lus Mhic Raonail (= Mac Ronald's wort), irlandais, CAMEron. [H. G.] jungfrun i det gröna, suédois, Grims.

jomfruen $i$ det grönne, jomfrugrön, gret' $i$ e grönn', danois dialectal,

JexNsen-Tusch, Nord. planten.

cernucha, (= la petite noire), tchèque, 1. Muelder, Synonym. Namen. crnika, curel, mohnnica, tamburica, zuećak, trofilic, vetrnica, serbocroate, Šulek, Juy. im. bilja.

carnilia, cjureli, bosniaque, LINDE, Sl. jez. polsk.

dylia tchernouchka, netchessany pannotchky (= demoiselles non peignées), petit russien, c. par M. Тн. VoLkov.

czarnucha, czarnuszka, polonais, LINDE

¿̇ydowa broda (= barbe de juif), wende, Schulenburg, Wendisches

lolksthum, 188:.

liè liandilla, magyar, Fusz, Trivialnamen. 


\section{FOLKLORE.}

1. Wenn ein Mädchen im Kanton Zurich Schabab einem Jüngling überreicht, so ist es ein Zeichen dasz sie ihn verschmäht.

Zeitschr. f. d. deutsche Myth. IV, 177.

2. On prend la nielle en boisson ì la dose d'une drachme contre les araignées.

Pline, XX, 71, (Trarluct. LitTré).

Cette médication est sans doute basée sur la doctrine de la signature; la plante ayant une certaine ressemblance avec l'araignée (voyez plus haut parmi les noms de la nielle), devait avoir pour vertu de guérir les morsures de cet animal.

\section{NIGELLA SATIVA. (LINNE). - LA NIELLE DES JARDINS.}

\section{1. - nOMS de Cette PLANTe.}

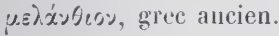

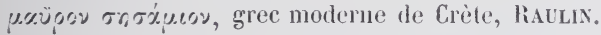

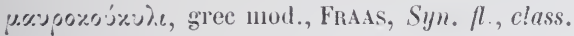

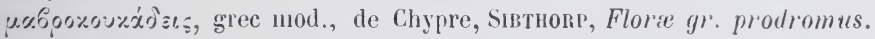

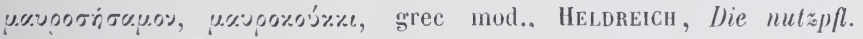

Griechenl., 1862.

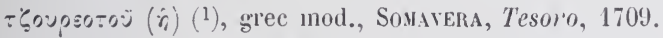

melanthium, latin, Columelle, (I ${ }^{\mathrm{e}}$ siècle ap. J. C.)

papaver nigrum, latin, DIOscoRIDE.

git, gith, latin, Pline, (Ier s. ap. J. C) ; Colunelle, (Irr s. ap. J. C.); Mak-

cellus Expiricus ( $1 \mathrm{~V}^{\mathrm{e}} \mathrm{s}$. ap. J. C.); Capitulaires de Charlemagne

(VIII s. ap. J. C.) ; DE Bosco, Luminare majus, 1490.

gitter, neutre, latiı du $\mathrm{VI}^{\mathrm{e}}$ siècle; dans une ancienne traduction latine, des ouvres médicales d'Alexandre de Tralde (méd. grec du VI ${ }^{*}$ s.) publice par Jacob de PARTleus, à Paris, en 1520, sous le titre de Pratica Alexandri. Le mot gitter s'y truuve passim et chaque fois il est traduit en note par nigella.

melanspermum, melanthion, lat., Puine.

melancium, ciminum ethiopum, ciminum ethiopicum, agrimatum, ugrimulalum, acrimulatum, latin du moyen àge, MowAT.

(1) Ce mot vient du nom turc de la nigella sativa cheurek ote. 
melantium, melachion, nigella nigra, anc. nomenclat., DE Bosc0, Luminare majus, 1490.

nigella romana, melantlium sativum, anc. nomenclature, B.uнı,, Pinax, 1671.

cumınum nigrum, anc. nomencl., Rosexthal, Syn. plant. diapll.

melaspermon, anc., nomencl., Duchesse, De Stirpilus, 1544.

nigella, nigella citrina, anc. nomenclat., DE Bosco, Luminare majus.

1490 , in-fol., fo $20, \mathrm{r}^{\circ}$.

ciminum nigrum, anc. $110 \mathrm{mencl}$, Aloxso, 1606.

nigelle de Crète, f. français, Bon jardinier pour 1811, p. 376.

nielle des jardins, f., nielle romuine, f., nielle de Candie, f., nielle du

levant, f., nielle cultivé, f., cumin noir, m., faux cumin, m., graine noire, f., her be aux épices, f., toute épice, f., quatre épices, f. pl., français.

niello dei jardin, f., Forcalquice, c. par M. E. PLAuchud.

piperelle, f., nielle citrine, f., anc. franç., Cotriare, Fr. dict., 1650.

poyrrade, f., anc. Iranc.. DEcAisie, Catal.

melantio, melanzio, melantro, nigella, nigella romana, nigella odorata, yitterone, italien.

gitono, italien, DE Bosco, Luminare majus. 1490, fo $20, \mathrm{r}^{\circ}$.

salusandria, italien, DeEz, Diz. ital. franz., 1678.

cimnamomea, cinnamomia, cardamomo tratto, cominella, cuminella, erba spezie, melantio domestico, ital., TARgiow.

papacero niyro, ital., Lexic. italico-gallicum, 1648, p. 111. - Padoue, PATRlarcili.

gittajone, ital., Arrivabene, Dizionario domestico, 1809.

giotton, milanais, BAxf, 「oc. mil.

spezii, Parme, Malispina, Vocab. parmig.

erba drogfa, milanais, Cherubini

scapiglieda, romagnol, MorR I.

geta, cheủsso, cösso, neirèt, piémontais, ZaLl, Diz. piem.

alpirre, alipirre, axenus (1), agenuz, neguilla sativa, espagnol.

neguilla, melancio, simiente negra, anc. espagn., Alovso, Privilegios, etc. 1660.

alipivre, portugais, GRISLEY, V'irilarium lusitanicum.

climen negru, negrushca, rommain, Braxdza, Limba bot.

cernuslıca, roumain, Ciнac, Dict. daco-rom.

(1) Ce mot rient de l'ancien arabe d'Espagne ach chenou. (Dozy, Supppl, aux dict. arabes). 
narden samen, zahme narden, schwar:er coriander, römischer coriander, schwurzhümmel, böhmischer schwarzhümmel, allemand. kimmel, Luxembourg, KolTz, Flore du Lux.

kreuzliümmel, bords de la Weser, Focke, Pflanzenn. - Prusse, Frischbier, Pr. Wört.

swarlen krülzkïm, Mecklembourg, Pritzel et Jess. Vollisn. de Pfl.

schwart komich, schwar $\approx$ coriander, ane. all., Cordus, Botanologicon, 1533. schwarzer liümich, schabab, morkrul, anc. aïem., iJEFENBACH, Gil. med.

lalin.

schwarz kimm, all. de Transylvanie, Fusz, Trivialn.

lioodlïiem (les semences de la plante), lookümel (idem), bas allemand, Focke, Pflanzenn.

narduszuad, hollandais, DoDoNaeus, Cruydl-boeck, 1644. [1. DE C.]. zwarte comyn, hollandais, Nexnich, Calholicon.

garden nigella, sweet flurour, bishop's wort, coriander of Rome, anglais, Cotgrave, French Dict., 1650.

bwlwg Rhufain (= bwlwg de Rome), llysiau 'r bara (= herbes dı pain), gallois, J. DaviEs, 1642. [H. G.)

buhwg lledfeyin (= bwlwg domestique), gallois, Meddygon Myddfai. [H.G.]. suartliommen, danois, Jfixsen-Tusch, Nord. pl.

cerny kmin, tehèque, Zaluziansks, Melhodi herbariae libri, 160i.

cernucha domaci, tchèque.

zamurski limen, zamorska liumina, serbo-croate. S̀lek.

Imine, l'nsse. [Th. V.].

lchernouchka (= la petite noire), petit russien, e. par М. Тн. VoLkoк.

vad koriandron, magyar, Fusz, Trivialn.

upahuntchikâ, ḳ̣shnadjîralia, ulkuntchikâ, säli, djarana, karavi, bahugandha, prthvi, sanscrit (2), Boенtlingk, Sanscr. Wört.

xamim, arabe, SÉrapion L'ANCIEx, Pratica Serapionis, 1525.

chouniz, ach-chouniz, arabe, FreYTAG.

chenou:, ach-chenouz, arabe d'Espagne, Dozy.

chouiniz, arabe syrien, Berggres, Guide francais-urabe.

sanoudj, sinoudj, arabe algérien, Duverrier, Les Tourregs, 1861 ; Leclerc, Révélations des énigmes, 1874.

hhabbet hellaua, arabe marocain, QuedenFELDT, p. ㄴ.17.

hhabbet es souda (= graine noire), arabe égyptien, DeliLe. - arabe syrien, Berggren, Guide. - arabe algérien, Duveyrier.

(1) Ces noms sanscrits sappliquent à la Nigella indica, Roxburgh, qui diffère peu de la Nigella sativa. 
kable soude, arabe égyptien, CLoT-BET, Аpercu sur l'Egypte.

kammoun asoùd (= cumin noir), arabe égyptien, DELILE, Flor. aegypt. ill., 1824. - arabe d'Audjìla, Ascherson, Norlafrica's Pfl., dans Rohly's Expedit., 1881.

hhebt el baraki, Nep, Russeld, Nat. hist. of Aleppo, 1794.

hhabbet el bourke, arabe syrien, BERgGren, Guide fr. ar.

sialılana, Turkestan oriental, SHAw, Sketch of turki lany., 1880.

ghaudona, sijadona (la semence), Turkestan, DragendorfF, P. 54.

difli, per'sa:, IDEy.

tcheureh ote, turc, Baribier de llesNard, Dict. turc.

asmul, amlarique, Harris, Highlands of Aethiopia, 1811.

๖. - Cette plante pousse à l'état spontané dans le sud de l'Europe et en Orient; dans le reste de l'Orient elle est cultivée. Elle sert aux usages suivants :

Die kleinen, scharf dreieckigen Früclitchen, die einen eigenthïmlichen, gewürzhafteı Geruch und ebensolchen, dabei stechend scharfen Geschmack besitzen, sind officinell, und wurden früher als gelind reizendes, Blähungen treibendes, auflösendes und diuretiches Mittel, besonders bei Verschleimungen, Würmern, Gelbsucht u. s. w. angewendet, sind jedoch jetzt nur mehr in der Thierheilkunde, sowie in Aegypten und im Orient allgemein als Gewïrz im Gebrauch, auch lässt sich ein fettes $0 \mathrm{el}$ aus ihnen gewinnen.

RoseNthal, synopsis plantarum diaph.

En Eggyte on sime les graines de cette plante sur le pain (d'après Olivier, Sonnini, ete. et les gâteaux après les avoir pulvérisées... elles leur donnent une saveur légèrement aromatique et passent pour faciliter la digestion et engraisser. Cet emploi est déjà mentionné dans l'Écriture (Esbras XXVlII, „5)... Dans l'Inde on se sert de la variété appelée Nigella indica...

MÉrat, Dict. des matières médicalcs.

Même usage en Russie el en Roumanic.

La nielle des blés s'appelle à Liège : froumatche fromage). Les enfants en mangent les graines auxquelles ils trouvent un goûl très agréable de fromage.

Comm. par 11, 0. CoLson.

$$
\text { ISOPYREIT THALICITROIDES. (LINNÉ). }
$$

tolldoche, all. de Bohême, Pritzel et Jess., Volksn.

doltocke, Silésie, IDEM.

doldocke, Prusse, E. MEIER, Preussens Pflanzenyatl. 1839. [J. C.]

Iurski fehol, mačji cvit, sası, pu亡̇arka, serbo-croate, Šulek. 


\section{HELLEBORLS (GenI' ). LINNÉ. - L'HELLEBORE \\ 1. - NOMs.}

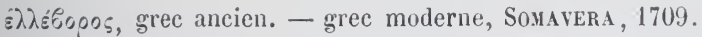

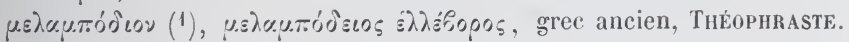

marifilium $\left({ }^{2}\right)$, grec, selon MatTh.seus Silvaticus, auteur du XIIIe s, cité par

Mowat.

dix́mupos, grec mod., Schnemer, Scriptores rei rusticae, t. II, ac partie, p. 320.

oxג̇pen, grec mod., Pouqueville, l'oy. de la Grèce, 1826; Sibthorp, Fl.

gr. prodr.; HeLdREICH, Nutzpfl. Griechenlands.

helleborum, helleborus, elleborus, latin.

veratrum, latin, Gaton (IIe s. av. J.-C.) ; Pline (I ${ }^{\text {er }}$ s. apr. J.-C.).

consiligo ( $\left.{ }^{3}\right)$, latin, Caton (II ${ }^{e}$ siècle av. J.-C.) ; VÉGÈCE (IVe s. ap. J.-C.). pulmonaria, radicula, latin, VÉGÉcE (IV s., ap. J.-C.), Mulomedicina,

édit. Schneider, page 151.

pulmonacea, latin, IDEM, p. 22.

gentiniana, aconita, latin du moyen âge, SchmelLer, Bayer. Wörterb.

faba lupina, marsuillum, marcilium, latin du moyen âge, Mowat, Alphita marsilium, lat. du moveen àge, Ger'nania, 1888, r. 307.

marcilia, folia lupina, Iatin du moyen âge, Bartholonaeus, cité par MoWAT. stradunella, velax, barba alfugi, ane. nomencI., DE Bosco, Luminare ma-

jus, $1496, \mathrm{f}^{\circ} 66, \mathrm{r}^{\circ}$.

helleborastrum, anc. nomenclat., Baunis, Pinax, 1671.

eleboire, m.. français du XV siècle, CAMus, L'opera salern., p. 63.

liellébore, f., ellébore, f., français.

ellebora, f, Nice, Risso, Hist. uat. des env. de Vice, 1826.

ellebre, français du XIITe s., Earle, Engl. plant-names, 1880, p. 45.

aliborgne, f., liborgue, f., liboüre, f., Meuse, Labourasse, Gloss.

aliboron, anc. fr., Roman du Reuard, édit. Mléon, vers 19309 (4).

(1) La découverte des vertus de cette plante est attribuée à Melampus. Voyez Puine, Hist. nat., $\mathrm{XXV}, 21,1$.

(2) C'est sans doute une faute de lecture, pour marisilium et le mot semble plutôt être latın.

(3) Sur le mot consiligo et sur son étymologie voyez la savante note de SCHNEıDER, dans son édition des Scriptores rei rusticae, 1797, tome II, 2e partie, p. 320.

(4) Voici le texte : "Aliboron i a trové / Que plusors genz ont esprové | Qui est bone por eschaufer, | Et por fièvres de cors oster. " | A une certaine époque, l'helleborum, corrompu en aliboron, était la panacée par excellence, préconisée par les charlatans. Par suite on a pu appeler Maitre Aliboron, un charlatan, un mauvais médecin, un ignorant, un âne (au figuré d'abord et finalement au propre). 
aliboro, f., Brive (Corrèze), G. DE LéPiNAY, Noms des plantes, 1886. liboro, f., Tulle, IDEM.

alibor, Toulouse, Tournon, Flore de Toulouse.

liborne, f., Vosges, Haillant, Fl. p. des V. - Ban de la Roche, Oberlin,

Descr. du B.-de-la-R., 1806. - Haute-Saòne, rec. pers.

lébore, f., lébôre, f., Vosges, Pétin, Dict. pat., 1842. - THiriat, Cata-

logue des végétaux employés, 1883.

ébôre, f., enr. de Domfront (Ome), c. par M. Iug. Chevalier.

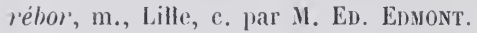

eubour, Ussel (Corrèze), G. de Lépinay, Noms des pl.

obre, anc. franç., Livocier, Hist. des pl., 158.1.

mar'sule ayjeste, anc. français, Reısscn, '. dans Arelhir de Herriğ,

1880, p. 175.

marsiouré, m., Gard. - Hérault. - Haute-Garomne. - Basses-Nipes.

siuré, m., (nom donné aussi ì l'euphorbe), mur-siur'é, m., (= euphorbe de mars (1), Lauraguais, c. par I. P. FagoT.

sidré, m., onest du Lot-et-Gar., c. par Il. l'abbé L. Daruy.

séré, m., Ancun (Hautes-Pyrénées), c. par M. M. Camélat.

marsioulé, m., Agenais, c, par II. l'abbé L. DARDY.

marsiéouré, m., Aveyron, Varssier. - Gard, comm. par M. P. Fesquet. morsioulé, m., Saint-Germain (Lot), Soulié.

morsiéoulé, m., Aveyron, VAYssier, Dict. lu pat.

marchïoulé, m., Brive (Corrèze), G. DE Lépinay, Noms des pl.

marsionné, m., Aveyron, Revue de l'Aveyron el du Lot.

marsouiré, m., Nimes, Vixcens, Topographie, 1802.

moursuble, m., maoussiblé, m., Apt, Colignon, Flore d'Apt.

moursiblé, m., mooussulble, m., provenc. mod., CAstor.

maoussiblé, m., Avignon, Palun, Ciatalogue, 1867.

massigoul, m., Tarn, GarY. - Sud-0uest du Languedoc, Duboul. -

Hérault, BARThès.

massirou, m., Gard, Rev. des langues rom., août 1884, p. 60.

muzeirro, m., muzsirroz, m., Suisse romande, Bridel, Gloss.; Durheim,

Schue. Idiot.

machar', m., Côte-d'Or, Roxer, Flore, 1881.

machère, Aube, Des Étangs, Noms des pl., 1844; Guénin, Statist. du canton des Riceys, 1852.

vérart, m., anc. fr., Duchesne, De Stirpibus, 15it.

vrare, m., Sallenches (Haute-Savoie), rec. personn.

(1) Par opposition aux véritables euphorbes qui fleurissent en été. - L'ellébore et l'euphorbe servent toutes deux à faire des sétons aux bestiaux, dans le Lauraguais. 
veraire, m., anc. fr.. Duchesne, De stirp.; Сн. Estienne, Dict., 1561 ; Cotgrave, 1650.

varaire, veraire, veraille, franc-comtois, DART0Is.

varaïré, m., Gard. - Hérault.

barairé, m., sud-ouest du Languedoc, Duboul. - canton de Murat (Can.

tal, Labouderie. - Hérault, Barthès.

boraüré, m., Cantal, J.-B. VEIRE, Piuoulats d'un reïpetit, 1860, p. 126.

vareyri, f., Forez, Gras, Dict. du pat.foréz., 1863.

veratrigno, f., Var, Amic, Considèr. s. Brignolles.

herbe ì la brochure, f., Centre, Jaubert, Gloss. - Allier, E. Olivier,

Flore pop. de l'Allier, 1886. - Cher, rec. pers.

brochure, f., Centre, Jaubert, Glossaire du Centre.

erba i la broticha, f., vaudois, DurHein, Schw. Id.

ierb' à la brotsche, f., brotze, f., Suisse rom., BRIDEL, Gloss.

rai à la brotza, f., brotza, f., Suisse romande, Durhen.

herbe ì l'encœur ('), f., encœur, Aube, Des Étangs, Noms des pl.

herbe aux boufs, f., Centre, Jaubert. - Anjou, Desvaux.

herbe aux bestiaux, f., herbe ì herber, f., Bocage normand, c. par

M. ED. Edmont.

herbe du feu, f., yèp di feu, f., wallon, Feller.

ièbe di fè, f., Orp-le-Grand (Belgique), Semertier.

herbe à sétons, f., Centre de la France. - Côte-d'Or. - Haute-Narne. -

Normandie.

herbe do creux, f., herbe do pointes, f., Vendée, Lalanne, Gloss. du pat. herbe du feu, f., Luxembourg, wallon, DAsNox, Dict. wallon, 1858.

erbo daou fio, f., Apt, Colignon, Fl. d'Apt. - Arles, Laugier de Char-

Trouse, Nomencl. pat.

erbo daou fué, f., provençal mod., Lioss, Végétaux utiles, 1863.

herbe d'enfer, f., Aube, Des Étangs, Noms des pl. - Guénin, Stat. du canton des Riceys.

enfer, Aube, Des Etangs, Noms des pl. de l'Aube.

lierbe à la rage $\left({ }^{2}\right)$, f., Manche, comm. par M. J. Courare du PARc.

herbe enragée, f., Avranchin, LitTré, Dict. s. vºllébor'e.

rage au loup, f., franc-comtois, DarToIs, Elude des patois, 1850.

raidge à loup, f., rèdge à loup, f., Montbéliard, Contejean. - Bournois,

(Doubs), Roussey.

(1) L'encour est une tumeur qui se développe spontanément au poitrail du bœuf et du cheval ou artificiellement au moyen de la racine d'ellébore. (DEs Étangs).

(2) Cette herbe sert à guérir une maladie des cochons appelée la rage qui n'a rien de commun avec la rage canine. (Couraye du PARc). 
poulmelée, f., pulmonée, f., anc. fr., Cotgrave, Fr. dict., 1650.

pommelaye, f., anc. français, Vinet et Mizauld, La Maison champêtre, 1607, p. 248.

pomelée, f., anç. franç., Cotgrave, 1650. - Hulsius, 1616.

poumeraie, f., Centre de la Fr., Jaubert, Gloss.

herbe i la pomilière, f., Plaine de Saint-André (Normandie), Joret, Flore. pomillière, f., canton de Périers (Manche), rec. pers.

poumillère, f., Guernesey, MÉTIVIER, Dict. franco-normand, 1870.

pomelière, f., poméliére, f., Bessin, Plunuet, Essai histor. sur Bayeux,

1829, p. 297. - Normandie, JoRET, Fl.

pommière, f., Bessin, Joret, Flore pop.

poumerelle, f., poumerolle, f., Nièvre, $\therefore$ par $\mathrm{A}$. Milliex.

herbe ì la purge, f., Centre, Jaubert, Gloss. - Semur (Côte-ıl'Or), c. par

M. H. Marlot.

contrepoison ( $\left.{ }^{1}\right)$, herbe au fi, Anjou, Desvadx, Fl. de l'Anj.

pisso-can, Gard, comm. par M. P. Fesquet.

pisso-co, m., Averron, Varssier.

pissacans, pl., Basses-Alpes, Annales des Basses-Alpes, 1838, p. 172.

pisso-ca, sud-ouest du Languedoc, Duboul.

pisse à chien, Allier, com. par M. C. BourgotgnoN.

pousse-neige, Aube, Des Etangs.

herbe i la rose, f., dans le Roumois (Normandie), JoRET, Flore.

rose dle Noël, f., rose d'hiver, f.. français.

rosa de Nadal, f., Pyrén. orient., Companyo, Hist. nat.

rose di Noue, f., Le Mans, Maclix, Plantes des env. du Mans.

rose ed Noé, f., Pas-de-Cal., comm. par M. Eo. Ednont.

rose de loup, f., Haute-Marne, comm. par M. A. Daguin.

rose des neiges, f., Loiret, c. par M. J. PORUET.

rose de serpent, f., Centre de la France, BonEau, $F l$.

herbe de Christ $\left.{ }^{2}\right)$, f., anc. franc., CotgRave, French. dict., 1650 ; DLEz, lict. frans. 1678.

christbloum (mot d'origine allemande), Ban de la Roche, H. G. OBERLix

Descript. 1806.

patte de lion, f., anc. fr., Decaisne ; Duez ; Hulsius.

pas de lion, m., Anjou, Desvaux, Flore de l'Anjou.

pied de yriffon, m., anc. franc., Vinet et Mizauld, La Maison champêtre, 1607 , p. 248.

(1) Contrepoison signifie double poison. (DESvaUX).

() Ainsi appelée parce qu'elle fleurit à Noël. (CotgRAve). 
griffon, anc. fr., dans divers mss. du moyen-âge. [J.-C.].

pè de griffon, m., Var, AMıc, Considérations.

peou de grifó, Pyrénées-0rient., Companyo, Hist. nat.

griffes du diable, f. pl., Loiret, comm. par M. L. Malon.

couyos de gat, f. pl., Luz (Hautes-Pyrénées), rec. personnellement.

bragos de loup, f. pl., Aveyron, Vayssier, Dict. du pat.

brago de lout, f., Laroquebrou (Cantal), c. par M. J. Malvezin.

vrailou, m., Les Fourgs (Doubs), Tissot, Patois des F., 1865.

queue au loup, f., fleur au loup, f., Doubs, BEauquier, Vocab.

pain du loup, m., IDEM.

pan u lô, m., Vallorbes (Suisse rom.), Vallotox-Aubert, Esquiss. de Val. pa de coulebros, m., Sud-ouest du Languedoc, Duboul.

pain de coü̈uvre, m., Lemmecourt (Vosges), Haillant, Fl. p.

arbe de quière, f., Tranqueville (Vosges), IDEM.

herbe à la couleuvre, f., pain de couleuvre, m., (Haute-Marne), comm. par M. A. DAGUiN.

herbe à porcs, f., Bocage et Avranchin (Normandie), JoRET, Flore pop.

herbe ed' Saint-Antoine, f., Saint-Pol (Pas-de-Cal.), c. par M. Ed. Edyont. fève de loup, f., Royer, Côte-d'Or, Flore.

fabo de lout, f., Lot, Puel, Catalogue.

fabo de loup, f., sud-ouest du Languedoc, Duboul.

fa'a au lau, f., pan au lau, m., Suisse romande, Vicat, Hist. des pl.vénén, 1776.

fava-lau, f., Suisse romande, Bridel, Gloss.

herbe à enfiler, f., Le Mans, Maulny, Pl. des env. du $M$.

cifonie, f., ( $\left.{ }^{1}\right)$, français du XVe siècle, Gachet, Gloss. du XV siècle, 1846.

cifoine, f., chiphoene, f., anc. franç., Godefroid.

sephoine, f., anc. franc., PAul Mexer, Deuxième rapport.

fayes du Sint Antône, f. pl., wallon, FELLER.

eleboro, starnuto, italien, Mesue, Libro della consolatione, 1575.

cavolo di lupo, elleboro, elabro, erba nocca, nocca, formes italiennes dialectales, TARGIONI, Diz. bot.

sebidiglia, sarde logodourien, SPANo.

anticircone, anticirone, anc. ital., Duez, Diz. ital. fr., 1678.

erba del lantcoéur, Parme, Malaspina, Vocab.

erba del lantcheur, Plaisance, Braccifortu, Fl. piac.

lèpro, Frioul, Pirona, Vocab. friul.

(1) La fleur de cette plante ressemble probablement à l'instrument de musique ancien de ce nom. 
cucs (les fleurs), IDEM.

dragunnha, Carpeneto, G. Ferraro, Bot. pop. di C'arpeneto.

branca lupina, fava del lupo, envir. de Pérouse, Zanetti, p. 181 et 195.

duracô, Côme, Monti.

nadéccia, romagnol, MoRRI.

fior de quaresima, Padoue, Patriarchi.

visciàtere, f. pl., lèburu, Abruzzes, Finamore.

erba besteira, anc. portugais, GRISLEY, Tiridarium, 1660.

eleboro, anc. espagnol.

toxico, yerva de ballesteros, anc. espagnol, Palmineno, Vocabulario del humanista, 1575.

yerba ballestera, espagnol, Colueiro, Dicc.

marxivols, catalan, Bull. de la Assoc. d'excurs. catalana, VIII, 232.

baladie, ancien catalan, Palmineno, Vocal)., 1575; Agustin, Libro de los secretos de agric., 1646.

cucurig, cutcurig bojotsei, roumain, KRANDzA, Limba bot.

hemera, anc. haut all., Diefenbach, Gil. lat. germ. m. lat.- PiPer, Altd pflanzenn.

emere, anc. haut all., Germania, 1881, p. 402.

wikgen, anc. haut all., Germania, 1888, p. 307.

suterwur\%, sittirwur\%, germar'un, germer, hemer, hemerwur\%, anc. all.,

DiefenbaCH, Gl. lat. germ. m. lat.

hemern, bavarois, Schmeller, Bayr. Wärt.

nieszwur*, bärenwur*, christwurzel, bärenfuss, allem.

wrangkrut, Mecklembourg, Pritz. et Jess., Vollisn. - Westphalie, LAN-

Dors, West. Pflanzenn. 1882.

wrangenkrut, Westphalie, LANDoIs.

wrangblom, Necklembourg, Pritz. el Jess., Volksn.

feuerkraut, eisblume, Prusse occident., Tпеichel, Volks.

schnecberger, Grosbliderstroff (Lorraine), rec. pers.

nieskruid, nieswortel, wrangwortel != herbe à sétons), hersenkruid, hol-

landais. [A. DE C.].

brandwortel ('), flamand. [A. DE C.].

oxnalib, setterwort, anglo-saxon, Cockayne, Leechdoms, etc.

lintwort, anc. angl., HuloEt, Dictionarie, 1572.

bear's foot, bearfoot, lungwort, setterwort, settergrasse, anglais, CoTGRAVE, Fr. dict., 1650.

(1) Ainsi nommée, parce qu'on l'emploie pour guérir les cochons malades du feu de SaintAntoine (brand $=$ feu). - [A. DE C.] 
fellonwort, boars'foot, barfoot (par suite de fausse étymologie populaire, pour bearfoot), anglais dialectal, Britten et Holl., Dict.

pegroots ('), anglais, Prior, Pop. names.

gargut-root $\left({ }^{2}\right)$, Norfolk, MaRshall, Rural economy of Norfolk, 1787.

troed yr arth (= pied d'ours), llewyg y llynger (= léthargie des lombrics),

llyngerllys (= herbe des lombries), pelydr ysbain (= pariétaire d'Espagne), torrgos (= gratte-ventre), torrlaeth (= lait de ventre), gallois, Meddygon Myddfai. |H. G.].

crafangc yr arth (= griffe d'ours), palf yr arth (= patte d'ours), gallois, Hugh Davies. [H. G.].

blaa-nollic (fleur de Noël), mannois, kELLY. [H. G.].

julerose (= rose de Noël), danois, c. par I. H. F. Feilberg.

bruluenn, breton, LAgadeuc, Le Catholicon. [E. E.].

burluenu, breton, LiÉGARd; Trovde. E. E.].

èvor, Le P. GRÉGoIRE. [E. E.].

čemerica, slovaque, MıkLosich, Slai. El. im. mag. p. 68.

cemerice, cemerka, keychawka, tchèque, LindE.

cemerizza, cemerikka, ragusien, IDEм.

cemerica, kukuvjek, bosniaque, IDEM.

zdravac, sprež̀, serbo-croate, Šulek, Juy. im. bilja.

čemerilia, serbe.

tchemeritza, russe, Mentzel, Lezicon pl. polygl., 1715.

tchemerytzia, petit russien, c. par M. Th. Volkov.

ciemierzyca, polonais, ŠuleK, Jug. im. bilja,

ciemiennik, polonais, LINDE, Slown. jez. polsk.

gemeric, lithuanien, Mentzel, Lexicon polygl.

tschemmura sahle, letton, STENDER, Lettisches Lexicon.

baladrea, basque labourdin, VAN Exs, Dict. basque.

lupu belar, basque espagnol. [J. V.]

kharbaq, arabe syrien, Berggren, Guide, 1844.

boinoz ote (= herbe à la corne), qaradja, (= noirâtre), tcheuplèmè, turc,

Barbier de Meinard, Dict. turc.

USAGES.

a). Sur l'usage médical de l'hellébore chez les anciens, voyez: DAREMrerG, Euvres d'Oribase, II, 800-806 et 809-810.

(1) From its roots being used by the cattle doctors in the operation of pegging or settering. (Prior).

(2) Gargut, = a disease incident to calves. 
Voyez encore l'article elleborisme par PInes, dans le t. V de l'Encyclopédie méthodique (médecine), p. 771 et suiv., enfin une note très importante dans SchneIder, Scriptores rei rusticae, 1796, t. II, Pars II, p. 320.

La racine d'Hellebore sert à siura les bêtes à cornes, surtout les bœufs. En introduisant un morceau de cette racine dans les plis de peau que fait naturellement le fanon, on obtient un engorgement énorme qui provoque un afflux de sang dérivatif souvent salutaire, mais il faut agir avec prudence, sous peine de voir la gangrène se produire.

Villefranche de Lauraguais (Haute-Garonne), c. par M. P. FAG0T.

b). Nos maréchaux guérissent les bestiaux malades d'une maladie appelée la pommelée dont le siège est dans le front entre les deux cornes de l'animal, en lui mettant de l'éllébore dans les narines.

Affiches du Poitou, 4 mai 1780.

Le suc de l'hellébore est employé par le vulgaire pour calmer le mal de dents et hâter la chute des dents gâtées. (Lot-et-Garonne), c. par M. l'abbé DARDY.

On emploie la racine d'hellébore contre les maux de dents, Semur (Côted'Or), c. par M. H. Marlot.

c) Voici ce que dit Clusius, sur la manière dont on peut reconnaitre la vraie pierre bezoar, contre-poison de l'hellébore :

«Inuenitur interdum Vlyfipone venalis hic lapis alia atque alia forma; quem tametsimagno admodum rstiment mercatores, ea conditione vendere nolunt vt periculum, an legitimus sit, faciat emptor. I 1 autem fit in hunc modum, ducitur cum acu filum per toxicum(herba balestera vocant) deinde per canis aut alterius animalculi pedem transmittitur acus, atque filum relinquitur in vulnere. Canis ilico ea symptomata incipit habere quæ comitari solent eos qui toxicum biberunt. Cum planè concidit canis atque desperatus videtur, tum puluerem ab hoc lapide abrasum et aqua dilutum cani in os injiciunt, si auxilium senserit canis, legitimi probatio est: sin minus, adulterinum censent) ».

Clusius, Aromatum historia, 1567, p. 191, en note.

Ballestera est le nom espagnol de l'hellébore (et aussi quelquefois d'une espèce voisine le veratrum album).

\section{LA POUDRE D'ORIBUS.}

Selon Lebrun, Etymologicon francois, feuillet 38, le mot poudre d'oribus serait une corruption de poudre d'elleborus.

« J'ay demandé et redemandé aux doctes médecins, quelle poudre c’étoit que la poudre d'oribus, mais ils n'en sçaroient rien. Et l'apprins en Allemaigne, les années passées au pays messein, pour en avoir vu chez les mer- 
ciers en petits cuyrs blancs de grosseur d'un doigt et autant de largeur, enfilez comme patenostres ( $\left.{ }^{1}\right)$. Et vendent cela aux bonnes gens comme remèdes à tous maux; dont est dict : contre toutes maladies il ne faut qu'un peu de poudre d'oribus. »

\section{FOLKLORE.}

1. - Sur l'ellébore chez les anciens, au point de vue iconographique et mythologique, voyez : Comptes rendus de la Commission archéol. de SaintPétersbourg pour 1865, in-fol., p. 90 et suiv.

Voyez encore : DAREMberg, Euvres d'Oribase, II, 796-797.

2. - Pour éloigner de l'écurie des porcs, les animaux malfaisants (salamandre, etc.) et ceux qui ont le mauvais oeil, il faut suspendre un pied de massivou, arraché au bord du chemin.

\section{Gari, comm. par P. FESQUET.}

0n suspend des rameaux d'hellébore dans les poulailler's pour éloigner la vermine, dans les étables pour purifier l'air et empêcher les maladies.

\section{Semur (Còte-d'Or), c. par M. H. Manlot.}

3. Un bouquet d'hellébore placé à la porte d'une jeune fille, le 1er mai, indique, comme symbole, qu'elle est intraitable.

Bournois (Doubs), Roussey, p. 203.

\section{HELLEBORL'S FETIDUS (LINNÉ). - L'HELLÉBORE}

\section{FÉTIDE $\left({ }^{2}\right)$.}

helleborus niger fotidus, pedicularia fotida, helleborastrum magnum, sesamoides magnum, pulmonaria Vegetii, helleborine major, helleboraster maximus, enneaphyllon Plinii, consiligo, ancienne nomenclature, Bauhis, Pinax, 1671.

héllébore fétide, français.

erba fétida, Pyrénées-0rientales, Companyo, Hist. nat.

réveil, m., Haute-Marne, comm. par M. A. DAGuis.

réveille-matin, Les Amognes (Indre), Jaubert, Gloss. du Centre.

ébore, lébore, alébône, pied de griffon, pas de corbeau, herbe à sétons, herbe à la bosse, herbe au fi, mords-cheval, galle, pomilière, Normandie, Joret. Fl. pop. de Norm.

(1) Comparez ci-rlessus, page 81 , le nom manceau de la plante : herbe à enfiler.

(2) Voyez l'article précédent dans lequel presque tous les noms donnés peuvent convenir l'Hellébore fétide. 
pomelière, f., Orne, LetacQ.

picha-can, m., Luchon (Pyrénées-Cientrales), Julien Sacaze, Flore pop. Menton, Rev. des trad. pop., 1894, p. 261.

pisse chien (1), m., herbe au bæuf, f., Lyonnais, Carriot. [Ed. Edм.]. picho-can, Lot-et-Garonne, c. par M. l'abbé L. DardY.

poison, herbe à arbi, f., pommeraie, f., parmeingnée, f., Haute-llarne, comm. par II. L. Aubriot.

biquette, f., Côte-d’Or, Rover, Flore.

erbè de la boba, f., Issoire (Puy-de-Dòme), comm. par M. J. B.ıreire.

marsegble, canton d'Aime (Savoie), comm. par M. MARjollet.

marsiouré, m., alibor, Sul-ouest du Languedoc, Duboul.

spuzzateste, Vérone, P'oldini, Flora veron.

elleboro puz:olente, ital., TARgioni, Diz. lot.

rizzot, Calabre, Tre mesi in Calabria, 1863, p. 36.

rizitta, rizzotà, Calabre, PASQUale et Arell., Flora di Napoli,1841.

munnolo, Pouille, IDEM.

campuren, erba de biss, rais de bo, Brescia, Zersi.

elleboro fetido, erva de besteiros, besteira, portugais, Brotero.

erbo do gando, galicien, Cuverno, Dicc. gall.

erba d'o gado, galicien, VAlLAdAREs.

marxibuls, catalan, Costa, Flora, 1864.

yerba llavera, eleborastro, espagnol, CoLmeiro, Dicc.

bárba-lúpului, roumain, HasDev.

meacan-sleibhe (= racine de montagne), irlandais, O'Reilly. [H. G.].

dahow duh, crub mahuin (= pied d'ours), irlandais, Threlkeld. [H. G.]. hylithr, hylyf, gallois, J. DAviEs, 1632. [H. G.].

\section{HELLEBORUS IIRIIISS (LINNÉ). - L'HELLÉBORE VERT (²).}

hellébore vert, m., hellébore à fleurs vertes, m., herbe à sétons, f., franc. encœu, Haute-Marne, comm. par II. L. Aubriot.

délivre (3), Loiret, comm. par II. L. MaLoN.

balandré, Argelès Pyrénées), comm. par M. P. TARissan.

herbe ed' Saint-Antoïgne (4), f., Boulonnais, comm. par M. B. dE KerHervé.

(1) Les chiens aiment à pisser sur cette plante.

(2) Voir ci-dessus les noms communs à tout le genre Helleborus.

(3) Dans le Loiret cette plante est employée pour la délivrance des vaches.

(4) Arec cette herbe les cultivateurs raccinent les porcs contre les épidémies. Boulonnais. [comm. par M. B. de Kerhervé] 
nocca da denti, elleboro verde, radicchiella, ital., TaRgioni, Diz. bot. radigle, radile, ardile, Frioul, Pirona. rizzitta, rizzota, Calabre, Pasquale et Arell., Flora di Napoli. munnolo, Pouille, IDEM.

calisson, Venise, A. Nardo Cibele, Zooloyia pop. veneta, 1887, p. 19,

(à propos des remèdes pour les bœufs'.

campuren vert, Brescia, ZERSI.

erba regia (1), Trévise, Siccardo, Flora trev.

oel-röstge, Thuringe, Pritzel et Jess., Volksn.

gillwurzen, Autriche et Silésie, IDEM.

schelmwurz, Linz (Autriche), IDEM.

göllkraut, gilbwurzel, Basse-Autriche, F. HoFER, Wörterb.

sier-cruydt, krop-cruydt, anc. flam., Dodoxaecs, 1641. 1. de C.]

elebor, gaélique écossais d'origine littéraire, CAMErox. [H. G.]

hvid pian, danois, JeNsSEN-Tusch, Nord. planten.

Bozistol (= chaise de Dieu), wende, Schulenburg.

kurji slep, (= cécité des poules), kurjica. serbo-croate, Šulek.

\section{HELLEBORUS NIGER (²). (LiNé). - L'HELLEBORE NOIR.}

veratrum atrum, latiı, CATON IIe s. ap. J.-C.)

veratrum nigrum, anc. nomenclature.

elleborus niger, elaborus niger, radicula, melampolion, poliorion, latin du Moyen-Age, Nowat.

alébore noire, f., anc. frans., (fin du XIVe siècle), La force et la nature les herbes, Bibl. nat., Paris, ms. 25328; "Prenez alebore noire et l'enveloppez en cire viẹge et le mettez sur la dent. » [J. C.].

elebore noir, m., anc. fr., Le jardin de santé, 1539.

hellébore noir, m., rose de .Voël, f., rose d'hiver, f., hellébore à fleurs roses, m., français.

roso de Nouve, f., Forcalquier (Basses-Ilpes), comm. par II. E. Placchud. arroso dé Nalaou, f., Ouest du Lot-et-Garonne, comm. par M. l'abbé L. DARDY.

(1) Regiar $i$ buo c'est se servir de cette plante comme remède pour guérir les bœufs de certaines maladies. (SAGcardo). Cette locution correspond à ce que lans le Centre de la France on appelle mettre l'harbe aux bestiaux, c'est-à-dire, selon JAUberT, leur faire un exutoire au moyen de cette plante.

(2) Voyez plus haut les noms communs à tout le genre Helleborus. 
verare noir, m., anc. franç., Pinaeus, Hist. plant., 1561. veraire noir, m., anc. fr., Cotgrave, Fr. Dict., 1650 ; Duez, Dict., 1678. demoiselle, f., Glisolles (Ouche, Normandie), Joret, Fl. p. de Norm. leit à baco (lait de vache), Sud-ouest du Languedoc, Duroul. pied de lion, m., Bocage normand, comm. par Ed. Edмont. starnuto negro, italien, Mesue, Libro della consolatione, 1475. elabro nero, elleboro nero, fau di lupo, piè di diavolo, tortelli di lupo, italien, TARgioni, Diz. bot. erba del mal zitôn, Bologne, Cononedi-Berti, Vorab. erba dragôn (1), Gênes, Casaccia, Diz. gen . campuren, Brescia, Zersi.

elleboru nigru, sicilien, Cupaxi, Hortus cath., 1696. vedegambro negro, rosa de Navidad, espagnol.

eleboro nigro, ravano gagisco, anc. espagn., Missheu, Dictionary, 1623. bozatsel $\left({ }^{2}\right)$, spinz, spintc, iarba nebulinor (3), roumain, Сıнас, Dict.

jarbe nebulinor, roumain de Transylvanic, Fusz, Trivialn.

ceaster aesc (4), ceaster u'yrt, hamorwyrt, anglo-saxon, Cockayxe. Christmas flower, anglais.

Christurur, allem. anc. et mod.

heiligen Cristwurtz, suterwurtze, anc. haut. all., Germania, 1888, p. 304. streifu'ur: anc. allem., Diefenbach, Gl. med. lat.

Christblume, Suisse allem., Durhein, Schw. It.

Kreschtblum, Luxembourg, J. WeBER.

Weihnachtsrose, Silésie, Pritzel et Jess., Volksn. schneerosen, hendl, schelmrosen, Carinthie, ZwAxziger.

schneekaderl, schneerose, krätzbleaml, gaugerwurz'n, Basse-Autriche, F. HOEFER.

starkwur*, Salzbourg, Nemich, Cathol.

heyligh Kerst-cruylt (sainte herbe de Noël), anc. flam., DodoraEus, 1644. [A. DE C.]

swart niescruydt, swarte nieswortel, flamand, Dodonaecs, 1641. [1. DE C.] pelydr $d u$, , = le noir pelydr; de l'anglais pellitory, qui signifie pariétaire), gallois, Hugh Davies. [H. G.]

(1) Usasi specialmente nella malattia detta il dragone, che sopraviiene alle vacche. (CASACCIA).

(2) Ainsi appelé à cause de sa ressemblance avec le boz (sureau yèble). (Ciriac).

(3) C'est-à-dire herbe des fous. (Cıнас). - On sait que les anciens se servaient de l'ellébore pour guérir la folie.

(4) Aesc $=$ fraxinus. 
cloerllys fawr (plante de la crevasse), gallois, Meddygon Myddfai. [H. G.] evor $d u$, breton, Le P. Grégoire [E. E.].

euor du, breton, Nomenclator. [E. E.] morosnik, russe, Schmalhausen. [Th. V.]

starodoubka, hor'ytzvit (= fleur brûlante), petit russien, с. par Тн. VoLKov. čemer̀ice črnú, hichavlia černá, elebor černy, Svateho Ducha kor̀en (= herbe du Saint-Esprit), tchèque, 1. MüLler, Synon. Namen. talog, petkoż, crnoglavak, zavlaćni lioren, devetik, alon, serbo-croate, ŠulEk, Jug. im bilja.

fekete hunyor, magyar, Clusius, Rar. pl. hist. 1601 ; Fusz, Trivialn. zaszpa, ligygo-fi, magyar, KoLbanı.

tapasvin, tiktâ, tîrê, la! sournalihân, arabe égyptien, Hussox, Syn. bot. arabe, 1845.

qara tcheuplemè, ture, BARBIER DE MEYNaRd, Dict. turc.

cara cioplema, ture, Sestini, Voyage dans la Grèce, 1789, p. 180.

cara ciopleme, turc, Pharmacopea româna, 1862.

\section{HELLEBORUS LIVIDUS. (AITON).}

palonia blanca, Baléares, Marès.

\section{HELLEBORUS PURPURASGENS. (WALdSTEIN).}

koailie popi, roumain transylvanien, Fusz, Trivialn.

lircheschlïssel, allemand transylvan., IDEx.

paiponya, magyar, IDEM.

fekete hunyor, fekete kükerts, magyar, KoLBANI.

\section{ERANTHIS HIEMALIS. (SAlisburi).}

helleborus hiemalis, nomenclat. de Linné.

helleborine, f., hellébore d'hiver, m., franç., Bon Jardinier pour 1811.

fleiran, provençal mod., Lions, Végétaux utiles.

elleborina, Vérone, PoLlini, Flora ver.

patrón, Parme, Malaspina, Vocab parm.

piè di gallo, italien, TARgioni, Diz. bot.

lentebloem, holl., Oudemans. [A. de C.]

kleyne geele wolfs-wortel, anc. flam., DodonaEus, 1644. [A. DE C.]

vinterblomme, munke, danois, JENSSEN-Tusch.

titra, ozimnica, bożični koren, serbo-croate, ŠULEK, Jug. i. b. 


\section{TROLLILS ELROI'EUS (LINNÉ). - LE TROLLE.}

NOMS DE CETTE PLANTE.

ranumculus montamus aconiti folio flore globoso, trollius flos, ranumculus globosus, ranunculus montanus, ranunculus montamus glomerotus, ranunculus alpinus luteus, ancienne nomenclature, ВАшнг, Pinax, 1671.

trolle, m., trolle globuleux, m., renoncule de montagne, f., français savant.

boule d'or, f., français vulg., c. par M. Eıl. EbMont.

boton de mizo, m., Suisse romande, Derhem, Schweiz. Idiot.

hllor de miazot, f., Suisse rom., Bridel, Glossaire.

boton d'or, m., Gerbamont (Vosges, Halllant, Fhore pop.

boutoun d'or', m., Forcalquier, comm. par II. E. PLacchud.

péndènt d'or, m., Luchon (Pyrénées Centrales), Julien SACaze. - Sudouest du Languedoc, Duboul.

coucourrous, provençal moderne, Liovs, Vég. utiles.

erba d'aou coucoum, f., Gard, Pouzouz, Flore du Gard.

bouquet de corbeau, m., Forez, A. Legrand, Stat. bot. du Forez, 1873.

narumculo de montayna, Vérone, Pollini, Flora veron.

bazi, Brescia, Zersi.

tarlupin, Frioul, PIroxa, Vocab. friul.

balbor, balboare, glonts, gloante, rommain, Cinac, Dict.

ylonta, roumain, BraxdzA, Limba botanica.

luumlsyifft, humlstorl, ancien allem., DIEFENBaCH, Gloss. kugelhahnenfuss, allemand, Rosenthal, Syn. pl. diaph.

trollblume, alphahnenfuss, allemand.

gelber alphanenfuss, engelblume, knollenblume, knoblemblume, linoble, dotterblume, yrosse glotzblume, melkerpappel, allemand, NЕмnicн. dodderblommen, allem. de Transylvanie, Fusz, Trivialn. ankenballe, canton de Schwitz, Pritzel et Jess., Volksn. poppenrolle, Grisons, IDEx.

engelblume, tönneni, töni, Suisse allem., DurheiM.

poparollen, Grisons, IDEM.

rolla, rugala, bergrolla, troldara, canton de Saint-Gall, Wartuass.

kuyelranunliel, Prusse, Frieschbier, Preuss. Wört.

runkeln, Prusse occid., Treichel.

butterblomen, butterrosen, kuliblumen, Carinthie, ZWANZIGER.

butterbleaml, mosblealm, riesenblume, budareseln, Basse-Autriche, F. HOEFER. 
älmkaibal, Styrie supérieure, IDEM.

drolbloem, hollandais.

globe flower, troll flower, anglais.

butter bump, butter-basket, goldy-locks, locker, lockyer goldens, witch

yowan, yolden-ball, formes dialectales anglaises, BRIt ten, Dict.

globes, West-Somerset, Elworthy.

bull jumpling, North Yorkshire, Notes and Queries, 28 nov. 1880.

gollin, Cumberland, Engl. Dial. Society, 1881, p. 109.

lucken yowan (= cabbage daisie), gaélique écossais, LigнтF0ot.

cronell ( = la boule), gallois, HugH DAvies. [H. G.]

bullerblomst, laxblomster, gulklokka, billerurta. ängbullar, pumpablomma,

brogummaknap, formes dialectales suédoises, Jexssex-Tısch, N. Pl. enyblomme, koblomme, kavalotlege, kabbeleje, kawling, aeggerlórel (= le jaune de l'œuf), Frejas guldrose, formes dialectales danoises, IDEMr.

balleblom, smürbolle, knappsolöj, slåtteblom, formes dial. norvég., IDEx.

kabbelotlege, lotkabbelege (= caltha fermé), danois. [H. F. F.]

valkullor (pour dvalkullor = assoupissement des filles. - C'est une des neuf fleurs qui tressées en guirlandes et mises sous l'oreiller d'une personne lui procurent un sommeil extatique, suédois, RıETz [H. F. Feilberg.]

úpolin, tchèque, A. Müller, Syn. namen.

planinčica, serbo-croate, Šulek, Juy. i. $b$.

pohrms, bula peere, letton, Ulmann, Lett. Wört.

a'dotki = petites Eudoxies), russe, Schmalmausex. [TH. V.].

kupalniza, russe, FALK, 1786.

jauczo burbulai, lithuanien, JАсовг, Lit. Pflanzenn.

boglaros torolya, magyar, Fisz, Trivialn.

pollo rose, lappon, Feldian, Index pl. fenn.

kuller kupud, esthonien, IDEs.

klupre kukka, finnois, IDEM.

\section{CALTHA PALUSTRIS (LINNÉ). - LE POPULAGE DES MARAIS}

\section{NOMS DE CETTE PLANTE.}

caltha ('), caltha palustris flore simplici, caltha poetarum, caltha Virgitii, chelidonia palustris, calendula, calendula simplici flore, chamaeleuce,

(1) L'ancienne nomenclature a souvent confondu sous ce nom (caltha) le populage et le souci (calendula). Les fleurs de ces deux plantes sont jaunes. 
ferraria, malacocissus major, epimedium Dodonaei, farfugium, populayo, ancienne nomenclature, Baunis, Pinax th. bot., 1671. populage, m., populage des marais, m., souci d'eau, m., souci des marais, $\mathrm{m} .$, grand bassin, m., français.

bachinet, m., chaudrons, m. pl., gaunel, m., gros jaunet, m., jauniot, m.,

Normandie, JoREt, Flore pop.

grand jaunet, canton de Vimoutiers (Orne), LETACQ.

bassinetz, m., ancien français, J. CAvus, Catalogue des $p l$.

bassineau, m., yollat, m., racanetle, Aube, Des Étaxgs, Noms des pl.

godat daw bon Diû, m., Meuse, Labourasse, Glossaire.

bassinot, m., bessinol, m., Vosges, Haillant, Fl. p. des $V$.

codron, m., Valenciennes, Héc.st, Dict. rouchi.

chaudron d'ôr, m., Spa, LEzAACK, Dicl. des noms wallons.

bassin d'aîu, m., wallon. SEMERTIER.

tchôdron, m., tchổron d'or', m., wallon, FeLLER .

pairouleto, f., (= petit chaudron), Sud-ouest du Languedoc, DLBoul. -

Hérault, Barthès.

clair bassin de rivière, m., cocussau, m., giron, m., Anjou, Desvadx.

bolon d'or, m., Vosges, Hailiant. Fl. pop. - Savoie, com. par M. M irjollet. gros bouton dor, m., souci d'ère, m., Char.-Inf., c. par M. E. Lemarié. ganelle, f., boulonnais, c. par M. B. DE KERHERVÉ.

jauneau, m., Haut-Maine, Montesson, Vocabul.

sourci d'eau, m., Haute-Narne, c. par M. A. Daguin.

gaouché d'ä̈go, m., provençal mod., Lioss, léy. utiles.

chaudière d'enfer, f., barbouillolle, f., Haute-Harne, c. par II. L. AUbRiot. buscateou, m., Basses-llpes, c. par M. E. Platchud.

pisse au lit, Meuse, Labourasse, Glossaire.

pilhtelé, Ban de la Roche, H. G. OBerlin, Description.

pichali, Luxembourg wallon, DAsNor, Dict. wallon.

p'lhotle de ch'vau, f., (= pissat de cheval), Chatel (Vosges), Haillant. péhholte dé chouî, f., Houssey (Vosges), IDEM.

poihholte dé ch'vau, f., Romont (Vosges), IDEx.

petle dé ch'vau, f., Vosges, IDEM.

pie de chevau, m., La Forge et Le Tholy (Vosges), IDEM.

poltes de tchevau, f. pl., Plancher-les-Mines (Haute-Saône), Poulet, 1878. gracilloun empoudoual (= cresson empoisonné), m., Luchon (Pyrénées),

Julien SACAZE.

graisselou empousounat, Sud-ouest du Languedoc, Duboul.

crbo de malfoundemén, f., erbo de morfoundemén, f., Ivejron, Vayssif.R.

erbo de malfoundomen, f. Aveyron, c. par $\mathbf{I}$. H. FaU.

fobrolo, f., Laroquebrou, Cantal, c. par M. J. MaLrezin. 
ardiol, Hérault, Barthès, Gloss. bot. - cévenol, Azais, Dict. des Idiomes. merla dzona, f., Château d'Oex (Suisse), Vicat, Plantes vénéneuses. touetoue, f., environs de Belfort, Rev'ue d'Alsace, 1887, p. 563. topotte (1), f., pototte, f., Montbéliard, ContejEax, Glossaire. pacoteyre, Bex (Suisse rom.), Durheim, Schw.-Id.. flour de mä̈, f., Briançonnais, comm. par I. J. A. Chabrand. lagagno, f., Ariège, comm. par I. A. JeAnroY. colomba, Moussey (Vosges), Halllant, Fl. pop. clivolonde, Géradmer (Vosges), IDEM. chevalon, chevanon, La Bresse (Vosges), IDEM. cocussot, m., Camembert (Hiémois, Normandie), JoREt, Fl. pop). cocu, m., Aube, Des Étangs, Noms des pl. - Haute-Narne, comm. par H. A. Daguin.

coucou, m., Aube, Des Etangs, Noms des pl. de l'Aube. calta palustre, farferugine, farferugio, margheritine gialle, italien, TARGIONi, Diz. bot.

narunculo d'acqua, Vérone, Pollini, Flora ver. cocleariót, Brescia, ZERSI.

flur da paing, Grisons et Engadine, Durhesi, Scluw.-Ill. violes grogues, anc. catalan, P0vio, Thesaurus puerilis, 1580. erba centella, galicien, VALLADARES. calcea calului, roumain, BRANDzA, Limba bot. bulbúc (mot à mot: bulle d'eau), calce, roumain, Сıндc, Dict. daco-roman. skeltschi, roumain de Transylvanie, Fusz, Trivialn. dotterblume, allemand. sumpf-smirgel, kuhblume, deutsche kapper, allem., Rosentнal, Syn. plant. beinblume, drahtblume, butterblume, allem., GRIмx.

polsterblum, schmâl๘blum, pelpes, m., frescheblum, Luxembourg, J.WEBER. goldblume, mattenblume, allemand, NEunich, Cath. georyirosen, kuhblumen, koliblumen, butterblumen, butterrosen, Carinthie, Zwanziger, Ver\%. 1888.

goldschmilhayel, Appenzell, Pritzel et Jess., Volksn. grosses pfändla, Memmingen, IDEM. riedrolle, canton des Grisons, IDEM. polpes, polsterblume, Eifel, Wirtgen, Vegetation der Eifel. buotterblome, Westphalie, LandoIs, Westf. Pflanzenn. kaublaume, Goettingue, Sснамвасн.

(1) Ainsi appelée parce qu'on peut produire un certain bruit avec une partie de la plante. (CONTEjean). 
dodderblommen, all. de Transylvanie, Fusz, Trivialn.

kugel-ranunkel, Kœnigsberg, Treiches, Polu. Westm.

kuhblume, Prusse orientale, IDEx.

mueterne, Grisons, Durhein, Schw. Idiot.

eierbluama, osterabluama, chrottabluama, ankablüomli, schmirblä, Canton

de Saint-Gall, Wartwass, Volksbot.

mosbleaml, Basse-Iutriche, F. HörER.

boter-bloem, flamand, DE GORTER.

lileine pompe-bloemen, Groningue, DE Gorter.

dotterbloem, lioll. et anc. flam., Dovonets et OudeMaxs. [1. de C.]

groote boterbloem, flamand, Rocces, Flore du Nord.

meerschboterbloem, meerschbloem, Flandre occidentale, DE Bo, Idioticon.

[A. DE C.].

puitbloem (= fleur aux grenouilles), palakiebloem, Flandre orientale, c.

par M. A. DE Cock

water-boterloloem, lileine plompen, holl., Oudemaxs. [A. DE C.].

marsh-marigold, anglais.

mariegold, brave basinet, anglais. Flonio, New world of words, 1611.

fiddles, Banffshire, Gregor, Dialect of Banffsh., 1866.

horse buttercop, drunkard, Deronshire, FniEx, Gloss. of Deronsh.

may blob, Sheffield, ADDr, Glossary, 1888.

blob, butterblob, mare blob, horse blob, moll blob, formes dialectales anglaises, Brittes, Dict.

water yoggles, IDEM.

marigold, fire-u'yold, IDEM.

meadow bright, cra $\vdots$, claut, IDEм.

boots, meadow bout, may flower's, Shropshire, JACksos, Word-book.

horse buttercup, West Somerset, ElWontнг, Word-book, 1886.

soldier's buttons, Somersetshire, Notes and Queries, 1887, p. 377.

willfire, Mearnshire (Ecosse), IDEM.

yellow gowan, Écosse, IDEM.

loppergowan, Clydeshire (Écosse), IDEM.

coulily, cowslops, meadow bultercup, Etats-Unis, BERGEN.

bearnan bealtine, lus bhuidhe bealtuinn, (= herbe jaune du $1^{\text {er }}$ mai), gaélique écossais, Cayerox. [H. G.].

bile buidhe (= plante jaune), gaélique écossais, Dict. of the Highland Soc.

[H. G.].

corrach shod, gaélique écossais, CAMERoN. [H. G.].

troed yr ebol (= pied de poulain), gallois de Llanrwst, J. WiLliams. [H. G.] gold y gors, (= gold du marais; gold est anglais), gallois, HugH DAvies. [H. G.]. 
ruddos y gors (= rougeâtre du marais), gallois de Cardiff, J. Storrie. [H. G.].

ruddos (= rougeâtre), gallois, WaLtens. [H. G.]. gold Mair (= gold de Marie; gold est le mot anglais), gallois, WaLters. [H. G.].

gold (c'est le mot anglais), gallois, WaLters. [H. G.].

synseg, gallois du comté de Breconshire, Waltens d'apr. le dict. de Th.

Richards. [H. G.].

lus Mairi (= Mary wort), irlandais, 0' ReIlls. H. G.].

plubairsin, irlandais, Threlkeld; 0’Reilly; - gaélique écossais, Dict. of

the Highland Soc. [H. G.].

sourcy dour, breton, P. GRÉGOIRE. [E. E.].

kabbalök, mjölkllomma, näkltuppor, peisktuppor, bälikeblomma, suédois

dialectal, JENSSEN-TusCH.

kabbalege, danois. [H. F. F.].

solijje, tremjöllisblom, hestehor, ryssehor', norvégien, Jexssex-Tuscr.

¿́askojes, didieji luksztai, lithuanien, ЈАсовг, Lit. Pflanzenn.

klingeri, illri, letton, HuPEL.

pluntschenes, spurna, purene, letton, Ulmax, Lett. Wör..

kaloujnitza, russe, Mentzel, Lexicon polygl.; Schмalhadsex. [Tu. V.]

latatie, !apli (= petites pattes), popyki (= petits popes, petits prêtres),

petit russien, comm. par M. Th. VoLkov.

woteoko (= ochsenauge), filsz, kniat, knieć, polonais de la Prusse, TreI-

CHEL, Poln. Vulgarn.

nogietek, kniecz, polonais, ERNDTEL, Warsavia illustrata.

majouka, polonais, Linde, Stown. jez. polsk.

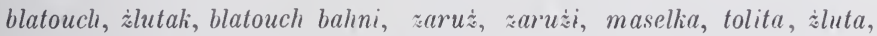

tchèque, A. Müller, Syn. namen.

maslenka, tchèque, ŠLleK, Jugosl. im. bilja.

zlatenica, slatica, slatocviet, żabnjak, veliki ¿̇uti żabljak, kalużina,

jagodnjak, djurdjevslio cveće, bielo zelje, mandalina spuga, volna

zlatica, potočni lapuh, ふ̇abokrečina, serbo-croate, Šulek.

motsári virág, magyar, Fusz, Vulgarn.

kelta kukka, wasikau sibna (= oculus vituli), finnois, FELLyan, Index pl. wassa kabiad, kuller kuppud, esthonien, IDEM.

\section{USAGES.}

Caltha plustris ist scharf und wird rom Vieh nicht gefressen, die Blumenknospen aber werden mit Essig eingemacht und als Kappern genossen, was jedoch kauın zu empfehlen sein dïrfte. Nach Niemann bereiten die Lapplän- 
der aus den gekochten Wurzeln Brod, was jedloch Linné und Wahlenberg nicht erwähnen.

Rosenthal, synopsis plant. diaph.

On se sert des fleurs de Caltha pour jaunir le beurre.

Pas-de-Calais, c. par M. B. DE Kerhervé.

3. FOLKLORE.

La première fois, au printemps, qu'on trait les vaches en pleins champs, on met dans le pot au lait nne fleur de caltha. Par suite le beurre réussira toute l'aunce et les sorcières ne pourront le voler.

\section{Danemark, J. KАмp. [H. F. F.]}

Vers le $\mathcal{L}^{\text {er }}$ mai, le pâtre du village distribue aux paysans propriẻtaires de bétail, des croix faites avec la fleur de caltha, une croix par chaque vache, en signe que les pàturages sont ì leur disposition. Ces croix sont suspendues aux pontres du plafond pour le reste de l’année.

Suède, Nicolovius. [H. F. F.]

\section{ACONITUM (1) (Genie). (LINNÉ). - L'AGONIT}

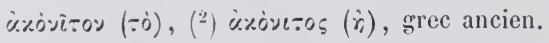

ن.\%óvго\%, grec mod., Somalera, Tesoro, 1709 ; Sibthorp, Fl. gr. prodr.

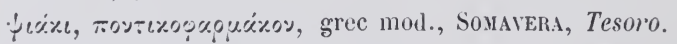

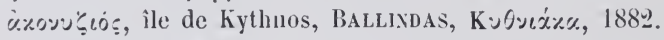

hecateis (3) ovidiana, anc. nomenclature, Duchesne, De Stirpibus, 1544.

aconitus, aconitum, luparia, ancienne nomenclature.

herba aconitana, bas latin, Arch. f. lat. Lexicogr., 1885, p. 470.

herba lupparia, nomencl. du XVe s., J. Canus, L'opera salern., p. 132.

cammoron, latin du XVIe s., J. Agricola, Medicinae herbariae libri duo, 1539 .

(1) Les différentes espèces d'aconit sont souvent confondues sous les mêmes noms. Cependant royez les articles suivants.

(2) Les anciens grecs se servaient de cette plante pour empoisonner les pointes de

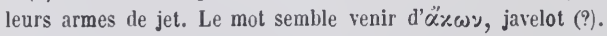

(3) Le mot hecateis qu'on trouve ainsi identifié avec l'aconit à l'époque de la Renaissance, se trouve dans Ovide, Métam., VI, 139. 
aconit, m., tue-loup, m., français.

estrangle lyepard, anc. franç., Pinaeus, Hist. plant. 1561.

estrangle loup, estrangle léopard, m., anc. français.

matallop, Pyrénées-0rient., Coupanyo, Hist. nat.

herbe au loup, f., français du XVIIe s., CotgRave, French. dict., 1650.

tora, f., Pyr.-Orient., Companyo, Hist. nat.

toro, f., languedocien, Sauvages. - Gard, c. par M. P. Fesquet.

tore, f., anc. franç., Duchesne, De stirpibus, 1544; Linocier, Hist. des plantes, 1583.

herbe de la tore, f., anc. franç., Duez, Dict. fr. ital. 1678.

touaro, f., provençal moderne, Lions, Vég. utiles.

pas de lion, m., Manche, J. Fleury, Essai sur le pat.

pé dé loup, m. Arrens (Hautes-Pyrénées, c. par M. M. CaMÉLAT.

pate louvine, f., anc. franç., Duchesne, De stirp., 1544.

pattes loupines, f. pl., J. Grévin, Deux livres des venins, 1568, p. 202.

toutchoz, Château d'0ex (Suisse rom.), Vicat, Plantes vénéneuses.

riagal, anc. franç., Broнon, De stirpibus, 15.41.

rigal, anc. franç., Duchesne, De stirp., 1544.

aconito, italien.

risayallo, ital., Lexicon ital. latin., 1648, p. 113

risigallo, sargalla, italien, SoMarera, Tesoro, 1709.

erba luparia, cancarina, sicilien, LAGUsi, Erbuario, 1742.

acónitu, acónete, accuonete, Abruzzes, Finamore, Bot. pop.

rejalyar, napelo, anc. espagnol, PaLminexo, Voc. del human ista, 1575.

centella, yerva de ballesteros, ancien espagnol.

anapelo, matalobus, espagnol anc. et mod.

erba verinosa, catalan, LACAVAlLeria, Ga:ophylacium catal. lat., 1696.

erba tora, acónit, catalan, Costa, Flora.

omag, omiac, oméy, tóie, roumain, Brandza, Limba bot.

thung ( ${ }^{1}$ ) anglo-saxon, Cockarie, Leechdoms, etc.

wolves bane, libbarls bane, anglais, Minsheu, Dictionary, 1623.

eitergift, moyen haut allemand, BENEcKE, Hittelhochdeutsches Wörterbuch.

liupewurz, anc. allem., PIIPE, Altd. Pflanzenn.

eiterwur;, luppewurz, egelgras, anc. allem., DiefENBACH, Gloss. germ.lat. sturmhut, wolfswurzel, wolfstod, hundstod, allemand.

(1) Sur ce nom anglo-saxon, voyez Hoops. p. 83-84. 
wolfskraut, lireutzkraut, sprossenliraut, allemand, Hulsius, Dict. fr. all. 1616.

gelstern, plur., bavarois, SchyelLen, Bayer. Wört

mänkekapp, Prusse, Frischbien, Preuss. Wört.

wolfswortel, inonnikslip, flamand et hollandais.

kurpgizelei, kurpijonai, lithuanien, JacoBY, Lit. Pflanzenn.

puchotel, russe, Mentzel, Lexicon polyglott.

borelz, (= lutteur), russe, Schmalhausen. [Th. V.]

zlymmiszeck, polonais, MENTZEL.

targes-tisché, mokschanien, Pallas, loyage en Russie, I, 110.

bîsch, khânek en noumr, arabe syrien, Berggren, Guide.

liessar el mouaa'n, lieng en nemeur, arabe algérien, Florian Pharaon,

Vocab. fr. ar., 1860.

\section{ACONITUM NAPELLUS' (LINNÉ). - L'ACONIT NAPEL.}

aconitum coruleum, aconitum lycoctonum cœruleum, napellus (1) anc. nomenclature, Bachus, Pinax, 1671.

cucullus monachi, luparia nigra. lupi radix, ancienne nomenclature, Geoffroy, Mat. méd., 1750.

aconit napel, m., aconit à fleurbleue, m., char de Vénus, m., français. fleur en casque, f., Bon jardinier pour 1827.

aconit à nat'et, m., napel bleu, m., français, Nemsich, Cathol.

cusque de pompier, m., Paris, rec. personnellement.

capuchon de moine, m., capuce de moine, madriettes, f. pl., the loup

bleu, napel, m., frangais, Geoffror, Mat. méd., 1750

napo, f., Toulon, Patout, Plantes médic. des environs de Toulon, 1864.

tore bluya, f., Gard, Pouzolz, Flore du Gard.

gueule dé leu, f., Valenciennes, HÉcart, Dict. rouchi.

sabots, m. pl., Ban de la Roche, H. G. Oberlin. - Vosges, Haillant. -

Wallon, Feller. - Haute-Marne, comm. par M. A. Daguin.

solè d' lieie d' préte (= souliers de cuir de prètre), La Forge et Le Tholy

(Vosges), Halllaxt, Fl. pop.

sabot d' préte, m., (Vosges), IDEM.

sola d'keu d' préte, Vallée de Cleurie (Vosges), Thiriat, Vallée de Cl.

pantoufes de Marie, f. pl., wallon, Feller.

siouet, m., Luchon (Pyrénées), Julien SACAze, Fl. pop.

(1) Sa racine a la forme d'un petit navet, de là vient le mot Napellus. 
coqueluchon, m., français, LoISELEur-DeslongChamps, Manuel des plantes usuelles.

erlo dou cascou, Forcalquier, c. par M. E. Plauchud.

casque, m., casque bleu, m., français.

bonnet d' yindarme, m. Herlin-le-Sec (Pas-de-Cal.), c. par M. Ed. Edront.

bâton royal, m. Ramecourt (Pas-de-Cal.), par M. Ed. Ediont.

garau bleu, m., Forez, A. Legrand, Stat. bot. du For.

picoutaz, L'Aigle (Suisse rom.), Vicat, Pl. vénén.

picouta, piouta, Alpes vaudoises. Durheim, Schu.-Idiot.

pistolets (1), m. pl., Haute-Marne, comm. par M. A. DAGuin.

pistoulets, m. pl., Haut-Boulonnais (Pas-de-Cal.), c. par M. B. DE Kerhervé. gants de la Bonne Damie, m. pl., Loiret, c. par M. J. P0QUET.

capuchon, m., capuchin, m., fleur au capucin, casque de Vénus, m., casquette, f. capet de gendarme, m., honnet d'évêque, m., chausson $\mathrm{m} .$, gants à la Bonne Vierge, m. pl., gueule de loup, f. tue-chien, petarls, in. pl., traquets m. pl., Normandie, JoRET, Flore pop.

bonette du méte, f. bonet de gendarme, m., wallon, FeLLer.

estranglo chi, Gard, comm. par M. P. FEsquet.

clotchas, f. pl. Ussel (Corrèze), G. DE LÉPINAY, Noms des pl.

lilokes, f. pl., wallon, Feller.

cou d' tchisse, m. (= hauts de chansse), cou d' tchâsse, m., tchôr di Vénus,

m., wallon, FELLER.

aconito, napello, malapelle, italien, TARgioni, Diz.

mapel, Brescia, Méchiori. - Vérone, Pollini. - Côme, Monti.

mapell, Mantoue, Cherubini.

napel, Vérone, Pollini, Flor'a veron.

palummedda, sicilien, LAGUSI, Erbuario, 1742.

anapelo azul, espagnol.

verdegambre azul, Grenade, BoIssier, Voy. lot. dans le midi de l'Esp.

erba tora, acónits, Vall de Nuria (Catalogne), Varreda, Catalech.

oméag, roumain, Сiнас, Dict. daco-roman.

omiak, roumain de Transylvanie, Fusz, Trivialn.

marul lupului (= pomme du loup), roumain, c. par M. A. GoRoveI.

blaue wolfswur\%, fuchswur:el, hundelod, giftwur, narrenkappe, mönchs-

liappe, kappenblume, liapuze, eisenhaut, blauer eisenhaut, eisen-

hütlein, blauer sturmhut, allemand.

teufelswurz, allemand, Rosenthal, Syn. plant. diaph.

fuchstodsturmhut, fuchswurz, allemand, GRIMI, Wörterb.

(1) Ainsi nommée à cause des pistils. 
duwenkutschen, Altmark, PritzFl et Jessen, Volksn. d. Pfl. schoiken, n., Gœttinguе, Sснамвасн.

blaue elster, Pinzgau, Pritzel et Jessen.

helmblom, fischerkip, duwenwayen, kutsch un peer, scholblom, Mecklembourg, IDEM.

mönkskappen, würgling, ziegentor, eisenhart, eisenhütel, Silésie, IDEM. gesellenschulichen, Prusse, Frischbier, Preuss. Wört.

isenhood, pâpmütz, pâpenmütz, pâtershappe, Frise orientale et environs

de Brême. Focke, Pllanzenu., 1870.

isühuat, bülına, kapuzinerchüppli, ritterspärli, wolfwörza, tübli = die

Nectarien), canton de Saint-Gall, Wartuaxi, Tollisholanili.

bloze, canton d'Appenzell, Durheis, Schw. Idiot.

malam, Grisons, IDEM.

fuchsblüah, teufelswurzn, täıberl im nest (1), Basse-1utriche, F. HöFER,

Esterr. pllanzenu. 1881.

tîsel (die honiggefiisse), (langage enfantin), Silésie, WesnнoLd, Beitr. $\approx u$ ein schles. Wörterb., 1855.

blauwe wolfswortel, muncliskapliens, anc. llam., DovonaEus, 1644.

[A. de C..].

palerskap, flamand, c. par M. A. de C.

aconite, blue aconite, monlishood, friar's cap, wolfsbane, blue wolfsbane, anglais.

helmet flower, great monkeshood, anglais, CotGRave, Fr. rlict.

monk's cowvl, cuckoo's caps, cat's tail, Shropshire, Jacksos, Hord-booli.

monkey's hood, parson in the pulpit, Devonshire, Fricxd, Gloss. of

Deronsh.

luckie's mutch, Lanarkshire (Ecosse), Janiesox, Etym. dict.

graulmother's nightcap, old granny's nightcap, old woman's nightcap,

old wive's mutches, cuctiold's cap, soldier's cap, turli's cap, monli's

cowl, anglais dialectal, Britten et HoLl., Dict. of plant-names.

face-in-hood, jacob's chariot, adam and eve $\left({ }^{2}\right)$, angl. dialect, IDEx.

bleidd-rlag (= étrangle-loup), blysiau 'r llaidd (= herbes lu loup), gallois, J. Davies, 1632. [H. G.].

bleiddan (= petit loup), bleiddlys (= plante du loup), gallois, Meddygon

Myddfai. [H. G.].

cwfl y mynach (trad. de l'angl. monk's cowl), gallois, HegH Davies [H.C.].

(1) Nach der Aehnlichkeit mit der Nektarien zwei Vugeln, die in dem helmformigen Kelchblatte wie in einem Netze sitzen. (HeFer).

(2) When the hood of the flower is lifted up, there is an appearance of two little figures. 
luss pyshoonag (= herbe empoisonnée), mannois, KELLY. [H. G.].

curaichd mhinaich (= monkshood), fualh mhadhaidh (= the wolf's aversion), gaélique écoss., Cameron. [H. G.]

cwcwll y mynach (= capuchon de moine), gallois de Cardiff, J. StorRIE.

[H. G.]

stormhalt, munkhatt, munkaskalle, suédois dialect., Jesssex-Tusch, Nord.

$p l$.

ulveurt, ulvebane, blämunke, munkekappe, venusvogn, blï duer, blü höns, töflelblomst, dronningetöfler, blikloklier, gifthätte, duesäde, duevogn, duekarme, träsko, oldefar a oldemor $i$ e kalis, jernurt, danois dialect., Іоем.

zlymiszeck (= le moine méchant), polonais, Mentzel, Lexicon polygl. morlownik, morderznik, mnisek sly, salomonek, polonais, LINDE, Slown.

jez. polsk.

morlownık (= meurtrier), russe, Nemnich, Cathol.

boret , petit russien, c. par M. Th. VoLkov.

jedhoj, tchèque, Šulek, Jugosl. im. bilja.

šlomonek, tchèque, Linde, Stown. jez. polsk.

nalep, naliep, nalip, jedic̈ dragoljub, krupiš, liupris, volina, vučji ëemer, ilka, lisjak (= herbe de renard), pesja smrt (= mort des chiens', preobjed, preobjedna, vrajnica, żelezni klobuk (= chapeau de fer), serbo-croate, šulEK.

irabelar, otseri, basque. [J. V.]

sisak fü, magyar, Fusz, Trivialnamen.

liatilia répa, magyar, Nemsich, Catholicon.

argoatka, toungouse, IDEM.

syath, Kamtschatka, IDEM.

bîch-moüch-bicha, quatel en nimr (= qui tue les panthères), quatel el dib, lihinek el dib, arabe, Iвx Beithar, traduct. Leclerc, I, 300; II. 2; III, 58.

peldirginu otu, turc, Pharmacopea romîna, 1862.

ACONITL.M LYCOCTONUM. (LINNÉ). -

\section{LACONIT TUE-LOUP.}

1. - Noms :

aconitum lycoctonum luteum, lycoctonum ponticum, aconitum luteum, ancienne nomenclature, Bauнis, Pinax, 1671.

vulpicida, vulparia, anc. nomenclature, Hexisch cité par Grimm, Deutches IVört. 
madriettes, f., pl., ancien franc. (d'après un ouvrage de 1556), Boucherie, Additions à Littré.

coqueluchon jaune, m., franç., Fillassier, 1791.

mort loüe, anc. franç., LE Bon, Etymologic. francois, 1571.

estranglo loup, Gard, c. par M. P. Fes@Uet. - Forcalquier, c. par M. E.

Plauchud.

toro jaouno, f., Gard, c. par M. P. Fesquet.

touera jaouna, f., provençal mod., Howvorat, Dict. prov., 18.18.

tout:o, m., Suisse rom., Bridel, Giloss,; Durneim, Schw. Idioticon.

yenn' cou d' chass' d'alman, Spa, LEzAACK, Noms wallons.

genne cou d'chausse d'allemand (= jaune eulotte d'allemand), Verviers,

Lejeune, Fl. de Spa.

fuelho de pèous, f., provenc. mod., AzAïs, Dict. des id. du midi.

erba della volpe, lupaja, strozsa lupo, ital., TARGioni, Diz.

piè de lor'o, Véronne, Pollin, Flora veron.

erba del lóv, Parme, Malaspixa, Voc parm.

erba de piagy, Brescia, Melchiori, Voc. bresc.

mata llop, Vall de Nuria (Catalogne), Virreda, Catalech.

yerva lupina, esp., Alosso, Privil. para mug. preñalas, 1606.

uva versa, uva lupina, espagn., J. Victor, Tesoro de las tres lenyuas. 1609.

yerva (= l'herbe par excellence), Grenade, Mayasin encyclop., an VI, 1). 504.

verlegambre, yerva de ballesteros, espagnol.

anapelo de Jarava, espagnoi, CoLmeiro, Dicc. gall.

gelber sturmhut, allemand.

weisse wolfswur $\%$, Carinthie, ZwANzIGER.

gelbe yelstern (1), Zillerthal, Pritz. et JEss.. Vollisn.

lusfloka, l'argdöl, suédois dial., JExssex-T., Tord. pl.

tirshjelm, torhat, troldhat, troldlijaerringhat, hundflok, norvégien, InEм. woltschei loren (= racine de loup), russe, FALK, Kenntn. d.russ. Reichs. pilirit, russe, Pallas, Voyaye en Russie, II, 266.

wilczi iad ziele ( $=$ herbe poison de loup), toiad ziele, polonais, Кемnich, Catholicon.

womêg, tchèque, IDEM.

crljivac̈a, omik, pesja smrt, rumeni preobjed, usjak, vuci koren, serbo-croate, Šulek.

farkas gyöker (= racine du loup), mergès fü (= herbe poison), magyar,

Nemich, Cathol.

(1) Galster $=$ Zauber. 
farkas-répa, farkas-bab, farkas mérey-fü, viszsza-forlulo-szölö, magyar, KOLBANi.

goncler, wotiak, FALK, Kenntn. d. russ. R.

ä̈oukoubsché (= herbe à l'ours), baschkir, PALlas, Voyage en Russie, II , 27.

\section{2. - USAGES.}

Die Wurzel dient zur Verg̣iftung von Wolfen, Hunden und Katzen, sowie zum Vertilgen der Ratten und Mäuse.

Rosenthal, Synopsis.

\section{AGONITU.U ANTHORA. (LINNÉ).}

\section{1. - Noys :}

antithora (1), anthora zedoaria, ancienne nomencl., Geoffror, Tr. de mat. méd., 1743.

anthora, aconitum salutiferum, anc. nomencl., B.1chIN, 1671.

actoire, français du XVe s., J. C.suus. L'Opera Sal., p. 36.

anthore, f., maclou, m., français, Geofrror, Traité de matière médic., 1743 , t. II, p. 14.

antitora, antora, aconito salutifero, italien.

antola, Vérone, Pollisi, Flora ver.

antoa, Gênes, Casaccia, Diz. genovese.

erba tora, Vall de Nuria (Catal.), Varreis, Ciatal.

aconito salutifero, espagnol.

omiak galbin, roumain transylvanien, lusz, Tririaln.

giel sturmhott, allemand transylv., lDEs.

gifftheil, allemand, Acarl. Leopold. nat. curios. Ephem., 1712, p. .9.

jadić, ljutić, ljutik, nalep, ċmerunlia, serbo-croate, Šlek, Juy. i. bilja. mniszek dobry, polonais, LINDE, Slown. jez. polst.

sirrga sisalfii, magyar, Fesz, Trivialnam.

schatlet es semm, arabe syrien, BERGgRen, Guide arabe.

antola saudâ, arabe, IBx Beithar, Trad. LeCLERC, I, 159.

\section{2. - USAGES.}

On fait souvent usage de la racine dans le Dauphiné pour faire mourir les vers et apaiser les tranchées des intestins.

Geoffrov, Traité de mat. méd., 1743, t. II, p. 16.

(1) Ainsi appelée parce quelle passait autrefois pour un antidote spécifique contre les autres aconits appelés thora. (Geoffroy). - Voyez, pour la synonymie, Mowat, Alphita, p. 10 , note 2 . 


\section{ACONITUM GAM.MARUM. (LINNÉ).}

aconitum magnum, anc. nomenclature.

aconit à grandes fleurs, français.

coqueluchon d'Allemagne, m. frangais, Fillissier, 1791.

cappa au moëno, f., bekla au moëno, f., Aigle (Suisse romande), Brudel, Glossaire.

bergsturmhut, allemand.

sisak-fü, kuklas-fu, tsuklya-fü, magyar, KoLBAxI.

\section{ACONITUM VARIEGATUM. (LINAÉ).}

aconit panaché, m., français.

eliaswagen, bords de la Weser, Focke, I'flanzenn.

peer' un urigen, rïter to peer, Brême, IDE..

gickel und tise, Silésie, Pritz. et Jess., Vollsn.

\section{ACONITLM FERON. (WALLIGH).}

\section{1. - Nous :}

ativisha (= surpoison, poison excessif), sanscrit.

bisch, arabe, Iвs Beїтhar, Trad. Leclerc, I, „98. - persan, Polak, Persien, 1865.

\section{2. - ISAGES.}

"Séclé, c’est un des aliments des gens du pays (le Halàlıil, en Chine, sur les Prontières de l'Inde), qui n'en éprouvent aucun accident. Mais quand on le sort de ce pars, seulement à la distance de cent pas, c'est un poison qui tue à l'instant celui qui en mange.

Ibs Semdjoun, cité par Ibn Beïthar, Trad. Leclerc, I, 298.

One of the most remarkable facts in the history of the aconit is that in certain localities its poisonous qualities are not developed, and it is so entirely innocuous that it is used as a potherb! That was pointed out as long ago as 1671 by Martin Bernhard, $\left.{ }^{1}\right)$, and was confirmed by Linnæus (2), so far as relates to Lapland, where the young shoots of one

(1) Napellus in Poloniâ non renenosus. - Ephem. Acad. Nat. Curios, Dec. i. ann. 2 (1671) 79.

(2) Flor. Lapponica, ed. 2. 1799. 187. 
species are cooked and eaten. It is still more strange to find that while in certain districts of Northern India the roots are collected as a poison, there are others in which the same roots are eaten as " a pleasant tonic (1."

Flückiger and Hanbury, Pharmacographia, Lond., 187: i, p. 8.

Bish is mentioned by the Persian physician Alhervi (2), in the 10th century as well as by Avicenna (3) and many other Arabian writers on medicine.

Upon the extinction of the Arabian school of medicine this virulent drug. seems to have fallen into oblivion. It is just named by Acosta (1578) as one of the ingredients of a pill which the Brahmin physicians give in fever and dysentery $\left({ }^{4}\right.$. There is also a very strange reference to it as "Bisch" in the Persian Pharmacopoia of Father Ange, where it is stated 5) that the root though most poisonous when fresh, is perfectly innocuous when dried, and that it is imported into Persia from lndia, and mixed with food and condiments as a restorative!

IDEMI.

L'aconit (napel) transplanté d'un lieu en un autre, par exemple des Alpes dans les jardins, perd sa qualité vénéneuse.

Voy. Geoffroy. Matière médicale. t. I, section II. page 121 (1750(1).

Aux étranges propriétés des diverses espèces d'aconits, ajoutez :

«L'aconite chasse le veniı hor's dı corps, mais s'il n'y en a point, il empoisonne. 》

LaUreNt Joubert, Erreurs popul., 1600. p. 135.

\section{3. - FOLKLORE.}

Pour la légende orientale de la belle jeune fille habituée dès son enfance a absorber ce poison, devenant renimeuse pour celui qui la touche ou la regarle et envoyée à certains ennemis de haut rang pour les faire périr sans qu'ils soient sur leurs gardes, voyez: W. Hertz, Sage ıom Giftmädchen (dans Abhandlungen d. l. bayer. Aku!. d. Wiss. Kl. I. XY. Bol. 1), 1893, p. 101-1 2 , 136-139, 140-151. L'auteur de ce curieux travail donne des détails sur les vertus toxiques des aconits, p. 136-139.

(1) Munro, quoted ly Hooker and Thomson, For. Ind. i. (1855) 58. 2nd part.

(2) Abu Mansur Mowafik ben Ali .llherui, Liber Fundamentorum Pharmacologix, i. (1830) 47. Seligmann's edition.

(3) Valgrisi edition, 1564. lib. ii. tract. 2. it. N. (p. 34ī).

(4) Clusius, Exotica, 288.

(5) Pharm. Persica, 1681. p. 358, also 17 and 319. 


\section{DELPHINIUM (') (GEXRE). - LE PIED D'ALOUETTE. 1. - Nous.}

consolida regalis, anc. nomenclature.

cornuta, cormueta, pes alaudie, latin du Noyen-Age, Diefexbacn, Gloss. med. lat.

flaura, latiı du moven-ìge, Germana, 1888, p. 205.

consoulde royale, $\mathrm{f}$., anc. français.

cornuette, f., anc. franç., DIEFexisacn, Gloss. med. lat; franģais de 1556,

Botchene, Add, à Littré, s. ro Cormuta.

cornetle, f., ane. franç., Chaune Holdet, Thécitre des jardinayes, Paris, 1678, p. 160.

cupouchin, m., capouchino, f., provençal moterne.

espuela de caballer, f., Pyrénées-0rient., Companyo, Hist. nulurelle.

esperonelle, f., ane. franç., J. Victon, Tesoro, 1609.

esporon, m., liegeois, l'orir, Dict. lièy., 1866.

sporon, m., Spa, Lezanck, Noms urallons des plantes.

éperon de la Vierge, m., Bocage normand, Lecoeur, Esqursse du Bocaye. époron d' chićralier, in., Valenriennes, Hécart.

pied d'alouette, m., français.

palte d'alonetle, f., Haute-Marne, comm. par II. A. Daguix. - Charente-

Inférieure, comm. par II. E. Lemarié.

pie d'ol'otle, in., pié d'alwate, in., pie d'olouette, m., pied d'ailouette,

m, pi d'alonalle, m., losges, Halldant, flore populaire.

pi d'olnevella, m., Suisse rom., lintdel, Glossaire.

pé d'ulonelte, m., Deux-Sivres, comm. par M. B. Socche.

pè d'uloneto, m., provençal moderne.

té de laonseto, m, Hérault, Barthès. - Languedocien, Duboll.

talon d'alonette, m., Bessin, Joret, Fl. populaire.

espéronn dé lirouzeta, ni., Luchon (Pỵténées), Jul. Sacaze.

pèd d'aloneto donble, pèd de carandro (= pied d'alouctte), Forcalquier

(Basses Alpes', e. par M. E. Plauchud.

pè de yat, m., espéroun de laonselo, m., Sud-oucst du Langued., Duroul. aloureto, f., Castres, Coczinıte, Dict. castrois. - Ouest du Lot-et-Gar., c. par M. L. DARDY.

(1) On confond souvent sous les mêmes noms les espèces delphinium consolida et delphinium ajacis. Les fleurs de ces plantes sont terminées par un éperon ou cornet aigu qu leur doine avant leur épanouissement, une grossière ressemblance arec un petit dauphin. (Bon jardinier pour 1811, p. 393 ). 
loneto, f., languedocien. Toursos, Flore de Toulonse. - Villefranche de Lauraguais Haute-Garonne), c. par II. Fagot.

pied d'oisean, m., patte d'oiseau, f., Haute-Marne, comm. par M. A. Dagun. pato d'aparrat, Montesquieu Haute-Garonne, c. par M. P. Fagot.

pè d'alóye, mi., patte d'oühè, f., wallon, Feller.

bec d'oiseau, m, français, L. Liger, Le Jardmier fleuriste, 1718, t. I, 86. dauphinelle, f., français.

dooufinenco, f. provençal mod., A. Boudis, Li set gorletto. 1879, p. 416. delfinette, f., framşais savant des jardiniers, Fildassıer, 1791.

dauplrinette. א., français, Bon jarlinier pour 1805.

lerbe aux poueils, f., Deux-Sèves, comm. par M. B. souche.

flous d'amour, f., Apt, Coligion, filore d'Apt.

flour de l'amour, f., environs d'Avignom, Paux, Catal.

damiséle, f., Landes, c. par II. l'abbé V. Forx.

riguligo, spron du caruliere, italien.

fior capuccio, Toseane, Caesalpinus, De plantis, 1583. - italien moderne. fior capizz, spron da caralir, romagnol, Moвв.

speron de cavalieri, ital, de Rome, P. Cistrubl, Hortus messanensis, 1610. speroni de caralleri, Sarlaigne du sud, Mons, Flora.

isprone de caraglieri, satrde logodumicn, spavo.

palumbedda, sicilien, LAgusi, Erbuario, 17 亿̇.

palummedda, sicilien, Copsx, IIortus cuth., 1696; Bıxcs, Flora d'Avola.

ricula del corno, milanais, Cherubini.

cornitte, Asti, Camisola, Flora uslese, 1854. [J. C.]

cournëlle, piémontais. Capello, llict. piém.

espuelas, Calella (Catalogne), Cuxi, Flor' 1883.

espuelas de caraller, Vall de Nuria (Catal., Virreds, Cutalech.

larkesfote, anglo-saxon, Cockirxe, Leechrloms, etc., 1860.

fuyelespyse, anglo-saxon, Diеfixiсн, Gl. med. la!.

knight's spur's, larlispur, anglais.

larlisheele, anglais, Cotgrave, F'rench dict., 1650.

ritters blomen, ritlerblum, rittersporen, anc. allem., Diefenвach, Gloss. rittersporn, allemand.

ritterspürichen, Leipzigg, ALввеснт, Die Leipziger Murrlart.

ridderspoor, hollamdais, flamand.

jiroliost, russe, Schmalhausen. [Th. V.]

redjel el imama, arabe algérien, Florian Pharaon, Vocub. franc. arabe;

Belkassem, Dialogues frang. arabe.

lihilef waladin, arabe syrien, BERGGRE, Gude arabe.

lalikum, arabe marocain, DoNBaY, Grammulica mauro-arab., 1800. 


\section{¿. - FOLKLOIE.}

1. Die Blumen alle Tag angesehen, wehren allem Iugenwehthmmb. Darumb hänken etliche büschlin von diesen blumen in ihre Gemach, dasz sie die stits im Gesicht haben.

Tyrol. - I. V. Zingerle, Einige Yotisen ans eimem Kraenterbnche.

2. Wer krank ist, lege in die sehuhe Rittersporn, dann wirds besser.

Transylvanie allemande. HALtricı, Z Zur Volksk. d. Siebenb. Sachsen, 1885, T. 297.

3. Man soll blane Rittersporn aus dem Kornfeld über die Stallthüre stecken, wenn danı die Truden kommen, spreehen sie : "hier simd blane Rittersporn, hier haben wir unsere Spm verloren, ) und die Thiere werden nicht geritten und nicht gequaiilt.

Tartlen (Transylranie allemande), HaLtuch, ldem.

\section{DELI'HINIU.M CONSOLIDA. (LINNÉ). LE PIED D'ALOUETTE DES CHLMPS.}

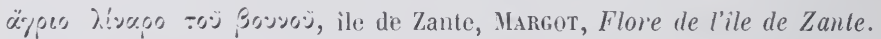
consolida regalis arvensis, consolida regia, flos regius sylvestris, cuminum sylvestre, delphinium sylvestre, delphinium vulyare, anthemis eranthemos, chanxmelum eranthemum, anc. nomenclature, B.ıuну, Pinax.

sanctre Othilix herba, anc. nomenclature. C. Baunin, De plantis a sanctis nотен halientibus, Basileac, 1591.

pied d'alonetle des champs, m., dauphinelle blene, f.. français. consolide royule, f., anc. franç.. Lixocier, IIist. des plantes, 1584.

consoulde royale, f., consoude royale, f., anc. français.

cumin sauvaye, m., anc. franç, Proprietez des simples, 1569, 1. 108.

ialousie, f., anc. frans., J. Cayus, Live d'heures.

flou d'amou, f., Gaid, comm. par II. P. Fesquet.

cormuette, f., anc. frauc. (dans un ouvage de 1556), Boccuerie, Addit. a Litlié.

yuilléoumé, m., Var, HaNrs, Calalogue. moine, m., Anjou, Desvaux, Flore.

bec d'oiseau, m., bec l'oisiau, m., bec à l'oiseau, m., bè d'oiseau, Centre, Jaubert, Gloss.

pique ù l'oisiau, f., Centre, IDEM. pekosi, Nyon (Suisse romande), Derherm, Schw. Idiol. roro blavo, Gard, comm. par M. P. Fesquet. 
beketta, f., Morat (Suisse rom.), Bridel, Giloss.

ristaperla (corruption de l'allemand rittersporn), Orbe (Suisse romande), Bridel, Gloss.

papè leurette, f., Issoire (Puy-de-Dòme), comm. par II. J. BAREIRE.

pas d'alouette, m., tête d'alouette, f., Normandie, Joret, Flore pop.

éperon de chevalier, français.

fiorcappuccio, consolida reale, consolida regale, italien.

cappucci, erba cornetta, ital., TARGIoNi, Diz.

spironelle salvareghe, Trévise, SAccardo, Fl. trev.

speronelle salvadeghe, cantayalleti, Vérone, PoLlisı, Flora.

viola del corno, Novare, Pollini, Flor'a veronensis.

erba de piagh, milanais, BANFi, Voc. mil.

syriff di passare, spironèle di champ, pid di pässare, Frioul, PIroNa,

Voc. friul.

espuela de caballero, espagnol.

consolda real, esporas bratas, portugais, Ввотево, Flora lnsit.

nemtishor, toporash, toporish, roumain, Brandz, Limba bot.

toporisek, roumain transylvanien, Fusz, Trivialn.

tatnish ('), nemerishori (plur.), pinten (= éperon), roumain, Сıнас. adebarsnibben, Mecklémbourg et Poméranie, PRitzel et Jess.

hornkümmel, Silésic, IDEM.

spuôrblum, Luxembourg, KoLTz. Flore.

schpuorblum, Luxembourg, J. WEBER.

lerchenklau', Prusse, Frischbier, Preuss. wört.

bloh rätterspühren, allem. de Transylvanie, Fusz, Trivialn.

rütersporen, in. s., Gottingue, Sснаншасн.

sankt Otilien kraut, allemand, G. R. BоEнмER, p. 5 2.

wilde ridderspooren, calketrip, flamand, DE GORTER.

larkspur, lark's heel, anglais.

yspardyn y marchog (= éperon du cavalier), gallois, HuGH DAvies. [H.G.] cornu, cornec, breton, Nomenclator. [E. E.]

riddarsporre, hömjöllia, suédois dialectal, Jenssen-Tusch, Nord. pl.

ridderspore, hanespore, vibetop, knop i kornet, danois dial., IDEM.

ostrożka, tchèque, ŠuleK, Jug. im bilja.

sualnik kralovstíg, liozi brádla (= petite barbe de chèvre), strači nůs̆ka,

tchèque, A. MüLler, Synon. namen.

oströkla, polonais, ŠulEk, Jug. im bilja.

modrzeniec, polonais de la Prusse, Treichel, Poln. Vulgärnamen.

(1) Ilu mot tata qui signifie père cest-à-dire religieux, prêtre. (Сrнac). 
madrzeniec, polonais, Lixde, Slou'n. jez. polsk.

kiavalerstä̈a clipora, (= éperon de cavalier), russe. [Th. V.]

éperon de chevalier. m., Pas de Calais, par M. ED. Ednont.

solirky (= petites hachettes), lomarory nossiliy (= becs de moustiques),

petit russien, comn. par M. Th. Volkov.

modrica, sralnic, ¿́arornjac, mamuzica (= éperon, konsul, sodula, ostrognica (= iperon , riterspor, serbo-croate, SulEk.

nehter, duhni, letton, Hupes.

pentinnas, lithuanien, CuHac, Dict. daco-rom.

lik sarkantyuvirag, magyar, Fus\%, Trivialn.

rooy, pilliroog, esthonien, HuPEL.

\section{DELPIINTIU AJAGIS ('). (LINNÉ). - LE PIED D'ALOUETTE DES JARDINS.}

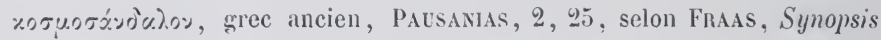
pl. Al. ciass.

grisuvò oov, grec byzantil, LANGKavel.

consolida regalis hortensis, calcatrippa, consolida reyalis flore rubro,

consolida reyalis sativa, flos regius flore purpureo, anc. nomenclat.,

Bauhis, Pinax, 1671.

sosandrum, anc. nomenclat., Duchesse, De stirpibus, 1544.

pied d'alouette des jardins, m., dauplinelle des jardins, f., français.

fleur royale, f., frans., Duez, Dict. fr. ital., 1678.

liéquette, f., frans. dialect., en Suisse?) Lamarck et Candolle.

herbe chaperonniere, $\mathrm{f}$., herbe moniule, f, ane. franç., Cotgrave, A french dict., 1550.

calcatrippa, cappucci, delfinio, fior capnccio, rigaligo, spazaa campayne, sprone di calaliere, ital., TARgioni, Diz.

smoinella, Plaisance, Bracciforti, Fl. piac.

speronèlu, Brescia, Zersi. - Padoue, Patriarchi.

spoü d'cavaler, Parme, Malaspina, loc. parm.

speron di caraliere, Vísuve, Pasquale, Flora vesuviana, 1869.

sperine de cavaliere, napolitain, fusumpaur.

speronelle, Vérone, l'olsixi, Flora ver.

spron cacaler, viola dal corno, lombard, loEs.

(1) A la base interne du pétale on observe trois ou quatre petites raies flexueuses où les Grecs avaient cru lire les lettres A I A, qui sont le commencement du nom d'Ajax. 
spron cavajer, piémontais, CoLLA, Ilerb. pedem. 1833. [J. C.]

spironèle, Frioul, Pirons, Voc, friul.

matapriogu, Sardaigne dı sud, Moris, Flora.

amô cornûo, Gênes, Casaccia, Diz. gen.

palometa, Baléares, Costa, Flora.

espuela de caballer, catalan, IDEM.

espuela de caballero, conejitos de los jardines, espagnol, CoLveiro, Dicc.

de los nombres.

espora de caballero, galicien, Cuveiro, Dicc. gall.

espuelas de caballero, galicien, Valladares.

consolda real, esporas dos jardins, portugais, BRotero, Fl. lusit.

surguciu, roumain, Brandza, Limbat bot.

delfin, brelon du Finistère, Crovan.

delfinez, breton, LIÉGARD.

solivliy, petit russien, [Th. V.]

aycilibouh, arabe égyptien, DeliLe, Fl. reyypt. ill.

\section{YARIÉTÉ CULTIVÉE.}

Il y a une variété qu'on appelle pied-d'alouette julienne ou nain, ou pyramidal, parce qu’il ne s'élève pas à plus de douze ou quinze pouces, et que sa tige est simple et lisposée en pyramide. Semé en corlon, il produit, par la diversité de ses nuances, un effet cliarmant, qu'on a pu voir, depuis quelques années, au Jardin des Tuileries.

Le Bon Jardinier pour 180 s.

DELPHINIUII STAPHISAGRIA. (LNXE).

\section{LA STAPHISAIGRE.}

rтup!s ivplx́, grec ancien, Dioscoride.

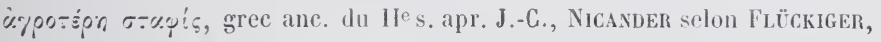
Pharmacographia.

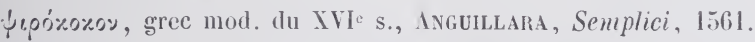

ùprortupiò, île de Zante, M.ırgot, Flove, I81.

†rocx́oikn, grec mod., Povqueville, Voy. de la Grèce.

perlicularis phtiroctonon, latiı, PLINE.

uva taminea, latin, CELse (Ier s. apr. J.-C.), selon MeIEr, Geschichte der Bot.

herba pedicularia, pedicularis herla, latin, Scribonius Largus (Ier s. apr. J.-C.), cité par MeIEn, Gesch. (l. Bot. 
slaphis agria, peduncularia $\left.{ }^{1}\right)$ (sic), latin, Marceluds Empiricus (fin du IV s. apr. J.-C.).

staphisagria, latin, Cassius, De medicinu (Ve s. apr. J.-C.). pituitaria, anc. nomenclat.. Duchesne, De stirpibus, $15 \mathbf{1 4 .}$

caputpurgium, pedicularis, anc. nomenclat., Syn. (dans Mesues, Compendium, 1623).

piper murium, piper glirium, granum pedicularium, delphinium platani folio, anc. nomenclat., Georfror, Matière médicale, 1750.

staphisagrie, f., stafisagrie, f., anc. français.

staphisaigre, f. $\left(^{2}\right)$, français.

estaphisayrie, f., anc. frane., Duchesne, De stirp., 1514.

stafisagre, anc. franç., J. Victor, Tesoro, 1609 ; Duez, Dicl.fr. ilal., 1678. estalizagro, f., Gard, Rev. des l. rom., aoùt 1881, p. 58.

estaflaigre, erbo des pevous, Forcalquier (Basses-Alpes), c. par M. E. I'LAUChud.

herbe a pouous, f., pedicnlaire, f., purgechief, anc. franç., I. Canus, L'Opera salern., p. 12us.

herle aux poux, f., mort aux poux, f., franc. anc. et moderne.

erbo eis pevous, f., Var, AuIc, Consid. sur Brignolles.

herbe aux pouillenx, f., anc. frang., Dobonscus, Herbarius, 1608.

herlue à la piluite, f., frans., Geofrror, Mat. médicale, t. III, section II, p. $1 \stackrel{0}{0 .}$

cilodillio, f., Corrèze, G. oE Lépinay, Noms des plantes.

aloue to tiio pouils, f., Ouest du Lot-et-Gar. c. par M. l'abbé L. DARDs.

pe d'alaoudo, m.. Landes, J. L̇̉on, Flore landaise, 1876.

stufisáyr'a, stufisuyria, italien mod.

stafisayria, stafisayra, strafizzeca, strafusagna, capopurgi, anc. italien,

Anbrosin, I'hytologia, 1666.

strafizeca, anc. italien, Hortus siccus A. Caesalpini, n 704. [J. G.] stafisagra, stafusaria, anc. ital., DuEz, Dict. fr., 1678.

strafusciria, anc. ital, Avbrosinı, Phytologia, 1566. - Milanais, Banfi,

Voc. milanais.

strafusare, Brescia, Zersi.

erba rla peochi, Venise, Boerio, Diz. - Padoue, Patriarchi.

erba da bduc', Bologne, Coronedi-Berti, Vocab.

erba p $i$ bdoce, romagnol, MorR.

erba da pighêuggi, Gênes, Casaccia, Diz. gen.

matupriógu, Sardaigne, PORRU, Diz. sardu, 1832.

(1) Corruption du mot pedicularia.

(2) Duchesne, De Stirp.. 1544, fait le mot masculin. 
ammazzapıógu, sarde logodourien, SPANo.

ammaz:apriogu, sarde méridional, Spano.

ammazzapidocciu, sarde septentrional, SPANo.

cabbarrasi (1), sicilien, Cupan, Hortus cath., 1696.

capurasu, crusta fidi, sicilien, LAGusi, Erbuario, 1742.

carràbaso, simenza di pidocchi, sicilien, BIANCA, Fl. dei dint. d’Avola,

1842.

matapoy, catalan, Costa, Flora. - Baléares, Marès, Catalogue.

havarras, yerva piojenta, espagnol, Palmireno, Voc. del humanista, 1575. abarraz $\left({ }^{2}\right)$, paparraz, favaraz, piogenta yerva, espagnol, Minsheu,

Dictionary, 1623.

erba piojera, coca piojera, albarraz, matapiojos, espagnol, Colmeiro, Dicc. erba piollenta, erba piolleira, galicien, VAlLadares.

paparraz, anc. portug., GRIsLer, Virilarium, 1660. - portug. mod.,

Brotero, Fl. lusit.

kopwurz, speichelhraut, lauswurtz, bys:müntz, berghicher, nismörder,

stephanskörner (3), anc. allem., Diefenbaci, Gloss. med. lat.

perchkicher, lü̈sliraut, anc. haut all., Germunia, 1875, p. 51.

laïsesamen, laüsekraut, allem., МЕмхгс, Cathol.

leishraut, Luxembourg, J. WeBER.

stavisagre, stavesacre, lowsebane, anc. angl., Musueu. Dict., 1623.

licebane, angl., Cotgrave, French dict., 1650.

Ilysiau' $r$ llau (= herbes aux poux), llysiau 'r poer = herbes à la salive

ou au crachat), gallois, J. DAvies, 1632. [H. G.].

lleulys, (= herhe aux poux), gallois, HuGH Daves. [H. G.].

lousaouenn-al-lıou, breton, Troude. [E. E.].

vusivka (= la pouilleuse), vus̈nica, uslivka, usiljivac, fraperc, prene.

znjača, serbo-croate, Šulek, Jug. im. bilja.

všivec (= le pouilleux), tchèque, IDEM.

aghitjak, arménien, Alishan. [Er. L.].

zorhibellıar, basque. [J. V.].

hlıabb er ras (= graine de la tête), arabe. - turc.

zerri'et el kamel, arabe de Nalte, Delicata, Flora melitensis.

zelib el djebel (= raisin sec de montagne), arabe syrien, BERGgren,

Guide, 1884. - arabe algérien, Florian Phara0n, Voc. ar.

mioukhaa', mioubazadj, araq ed daoueyt, arabe syrien, BerGGRen, Guide ar.

(1) Ce mot vient de hhabb ar ras (= graine de la tête), nom arabe de la staphisaigre.

(2) Ce nom et le suivant viennent de l'arabe hhabb ar ras.

(3) Il y a sans doute fausse étymologie populaire par suite de la confusion avec le mot staphisagria. 


\section{LSAGES.}

On fait avec la staphisaigre une poudre pour détruire les poux appelée : poudre de capucin, f., français.

graines des capucins, f. pl., Pas-de-Cal., c. par M. Ed. Edmont.

simince di capucin, f., simince di piou, f., simince di sporon, f., wallon, SEMERTIER.

strafusari, milanais, Cherubini.

haowerstoff, Altmark, DANneIL.

\section{DELPHINIU.M CaRDIOPETALU.I. (De Gandolee).}

bañeta, Baléares, MARÈs, Catal.

IELPIINIUM LONGIPES. (MoR.)

occhio di pupa, Barletta, Brunı.

siek el hamiema, Malte, Delucata, Fl. melit.

\section{IELPHINIU.M JUNGEU.I. (DE GANDOLLE).}

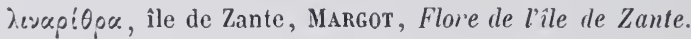

\section{DELPHINIUII ELATUM. (LINNÉ).}

pied d'aloutte vivace, m., franç., Bon Jardinier pour 1827.

grand pied dalouette vivace, m., delphinette de montagne, f., pied de mouche-abeille, m., franc., Fillassier, 1791.

tzar-zillé (= plante-roi ), petit russien, comm. par M. Th. Volkov.

\section{AOUILEGIA I'ULGARIS. (LINNÉ). - L'ANCOLIE.}

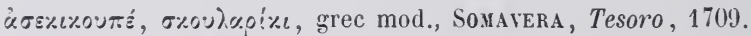

aquilea, lat. du Moyen-Age, Du CANGE, Gloss. med. lat.

alkaleia, lat. du Moyen-Age, Diefenbach, Gloss. med. lat.

ackeleia, acoleia, agleya, latin du XIIe s., Descener, Santa Ildegarda.

aquilegia, (') aquileia, aquilina, columbina, anc. nomencl., Bauhs, Pinax, 1671.

(1) Les diverses parties de la fleur représentent grossièrement un aigle, de là le nom d'Aquilegia. - On y a aussi vu une colombe. 
gants, m. pl., gants de Notre-Dame, m. pl., gants Notre-Dame, m. pl., français.

gants de la Vierge, m. pl., Orne, Letace.

gants de la Bonne-Vierge, m. pl., gants à la Vierge, m. pl., Normandie,

JoRET, Flore pop.

gants d'évêque, m. pl., Jouy en Pithiverais (Loiret), c. par M. J. Poquet. cinq doigts, Haute-Marne, comm. par M. A. Daguin.

chin deis (= cinq doigts), chinq dès, Manche, Fleurr, Essai sur le patois norm. - Avranchin, JoRET, Flore pop.

ditèra (= doigt de gant), f., Luchon (Pyrénées), Jul. SaCaze.

dé d' Notre-Dame, m., wallon, Feller.

sabot, m., Spa, LaZAack, Noms wallons des $p l$.

souliers du bon Dieu, m. pl., Haute-Marne, comm. par I. L. Aubriot.

- Aube, Bacdorn.

souléro, m., Aube, Des Étangs, Noms des pl.; GuÉnin, Statist. des Riceys. cornette, f., Oise, Graves, Catal. des pl. de l'Oise.

culotte, f., Aube, Des Etangs, Noms des pl. - Oise, Graves, Catal. des plantes.

côniau, m., marmites, f. pl., départ. de la Mayenne, comm. par un botaniste de la Mayenne.

gonnot, m., gonneau, m., Anjou, Desvaux, Fl. de l'Anjou. - Camembert

(Normandie), JoRET, Fl. pop.

clochettes f. pl., Maine. - Anjou. - Côte-d'Or. - Doubs. - Haute-

Marne. - Orne.

cloques, f. pl., Verquigneul (P.-de-Cal.), comm. par M. Ed. EDмoxt.

cloches, f. pl., Loiret (Eure et-Loir), c. par M. J. Poøuer.

kiaetchattes, f. pl., Ban-de-la-Roche, H. G. Oberuin, Descript., 1806.

cûchottes, f. pl., Ville-sur-Illon (Vosges, comm. par II. L. LECLERC.

franke chiyettes, 1. pl. (= clochettes franches), wallon, FELLER.

tionche, f. (= cloche), Lemmecourt (Vosges), Haillant, Fl. pop.

cloutseto, f., Brive, (Corrèze), G. DE LÉPINAY, Noms des pl.

campana, f., Tulle (Corrèze), IDEM.

campanes, f. pl., Pyrénées-0rient., Companyo, Hist. nat.

campanetos, f. pl., Hérault, Barthès, Giloss. bot. - Argelès Haute-Ga-

ronne), c. par II. P. TARRissan.

componetos, f. pl., Aveyron, Vayssier, Vocab.

gobelet, Jouy en Pith. Loiret, c. par I. J. PoQuer.

ptits valets, m. pl. (= petits garçons), wallon, FELLER.

bonne femme, f. Anjou, Desvaux, Flore.

veuves, t. pl., Bain (Ille-et-Vilaine), Orain, Glossaire.

viuda, f., Pyrénées-0rient., Companyo, Hist. nat. 
pénitént, m., Aveyron, Varssier, Vocab.

mounè, m., Somme, A. Ledieu.

capucine f., bounet de prétre, m., Charente-Inférieure, comm. par

M. E. LEMARIE்.

erbo de Nouestré-Dame, f., Bouches-du-Rhòne, Villexeuve, Statist.

chansons, f. pl., anc. franç., ReNé FraxçoIs, Essa!j des merveilles de la nature, 1622, p. 266.

tchassé, kinterrei, Suisse rom., BRIDEL, Gloss.

aquilée, f., uquilleee, f., anc. Iranç, Du Caxge, Giloss. med. lat.; J. Camus,

Réceptaire, p. 5; Reistsch, A. de Coutances, Arch. de Herrig, 1880, p. 172.

anquelie, f., anc. franç., Vax Hasselt, Ess. s. l'hist. de la poésie franc. en Belgique, 1838 , p. 185.

angorie, f, anc. franç., Scheler, Dictionn. étymol.

ancolie, f., français anc. et moderne.

ancoulio, f. Sud-Ouest du Languedoc, Duboll.

ancoile, f., anc. franc., Dovoexs, Herbarius, 1608.

ancolette, f., rancolette, f., acolette, f, icoletle, f., wallon, FELLER.

aiglantıno, f., languedocien, Mistral.

englantino, f., languedocien, DuвocL.

anglantino, f., églantino, f., sud-ouest du Languedoc, Duвoll.

yalantina, f., Gard, Pouzouz.

galuntino, f., Gard, comm. par M. P. FFsQUet. - Var, AMIC. - Apt

(Vaucluse), Coligiox. - Basses-Alpes, c. par II. E. Platchud. galatino, Var, HANRY.

colique, f., Saint-Pol, (Pas-de-Calais), comm. par M. Ed. Edмoxt.

poule, f., Tranqueville (Vosges), Haillaxt, Fl. pop.

coquelicot, m., Ramecourt P.-de-Cal., comm. par II. Ed. Enynxt.

dos de lou (= lents de loup), pl., Vagney (Vosges), Iderr.

griffe, Pạs d Auge (Normandie), Joret, Flore pop.

piboulette, f., Issoire (Puy-de-Dôme), comm. par M. J. Bareire.

erbe carmailhere, f., erbe clarèle, f., Landes, comm. par M. J. DE

LAPORTERIE.

mount' al cel = monte au cre.), sud-ouest du Languedoc, Duboul.

aquilegia, italien.

aquinegia, piémontais, CapflLo.

aquilina, lombard, ScaxinagatTi, 1794.

amor nascosto, fior cappuccio, italien, TARgioxi.

perfetto amore, italien de Rome, au XVII siècle, P. Castelli, Hortus messanensis, 1640. - italien moderne.

soccoli, vénitien, Vox Martexs, II, 91. 
brayhesse da prete (1), Trévise, Saccardo, Fl. trevig. scarpette, Vérone, Pollini, Fl. ver.

calse e braghe, Briesca, Melchioni, Voc. bresc.

acuilège, campanélis, Frioul, PIRoNA, Voc. friul.

pindagghi di regina, sicilien, CcPaNi, Hortus cath., 1696.

pinnágghi di rigina, sicilien, Mortillaro.

galeti, Padoue, Patriarchi.

aguileña, yuileña, pajarilla, pelicanos, manto real, clérigos boca abajo, espagnol.

aucellets, Minorque, Costa, Flora. - Vall de Nuria (Catal.), Varreda, Catalech.

perfet amor, Vall de Nuria, Vayreva, Cat.

amor perfecte, Calella (Catalogne, Cuxi, Flor'a, 1883.

paxariños, m. pl., galicien, luveıro, Dicc. gall.

aquilegia, erba pombinha, portugais, Brotero, Fl. lusil.

amor perfeito, anc. portugais, GRIsLEx, liridarium lusitanicum, caldarusha $(=$ petit chaudron), roumain, Сıнас, Dict. Iaco-rom.

tschintsch klopotzialie, tschintsch koadie, roumain de Transylvanie, Ftsz,

Trivialn.

gloie, gleie, moyen haut allemand, ВЕхескE, Mittelhochd. Würt.

tyriacksliraut, anc. allem., Dodoxaecs, 1614. [1. de C.].

ackeley, aglei, unserer Lieben Frauen handschuh, allemand.

goldwurzel, Luxembourg, KoLTz, Flore.

phïse, Suisse, Pritzel et Jess.

manzelebhume, Suisse, StaLder,

kilockenblome, klockjes, bas allem., Focke, Pflanzenn.

kinoliendè, Altmark, Daxiseı.

ylöckchen, Prusse occident., Treiches.

lilokkeblomme, allem. de Transilvanic, Fisz, Trivialı.

narrakappa, hapuzinerhïetli, fürbara, ylocka, schlotterhosa, canton de

Saint-Gall, Wartuan, Volksbotan.

schweizerhose, Suisse allem., DurHeIn, Schw. IJ.

drijakel, aglei, Prusse, Frischbier, Pr. wört.

handschuh, Basse-Iutriche, F. HöFER, Esterr. I'flanzenn.

alielei, aliolei, klokbloem, pantoffeltje, hollandais. VAN DALE, Hoordenb.

[1. de C.j.

acoleie, anc. flamand, SchelER, Dict. étym.

(1) Perché questo fiore ricurda iu qualche modo una mezza braghessa increspata. SACCARDO). 
akelei, kapelleken, llamand, c. par 11. А. DE Соск. cockesfot, anglo-saxon, Cockarie, Leechdoms, etc. setwall, columbine, anglais, Florio, New world of words, 1611. culverwort ( $\left.{ }^{1}\right)$, anglais, PRIOR, Pop. names. lady's shoes, lady's slipper, boots and shoes, hen and chickens (), dore's foot, colourbine (3), anglais dialectal, Britrex, Dict. snap dragon, yramie's nightcap, Devonshire, Friend, Gloss. of Dev. troed y ceiliog (= pied de coq, troed y ylommen (= pied de colombe), columbein du lat. columbinus ou de l'anglais columbine, Ilysiau ' $r$ cwlm (= herbes de la colombe), gallois, J. DAvies, 1632. [H. G.]. madwysg, gallois, Hugh Davies. [H. G.].

lus a cholamain (= dove’s plant), gaélique écoss., CAneros. [H. G.].

cruba leisin (= crouching of the thigh or haunch), lusan cholam (= herbe de la colombe). irlandais, Camerox. [H. G.].

akkeley, tyskaklokior, blakniljor, suédois dialectal, JENssEn-Tusch, Norıl. planten.

femfingerboelsurter, klokker, venusvogn, danois dialectal, IDEM. alikeleje, oldemor's natluer (= bonnet de nuit de grand mère), danois, c. par .I. H. F. Feilberg.

orliček, vorliček (= petit aigle), tchèque, 1. Müller, Syn. Namen. orlik (= petit aigle), polonais, Linde, Slown. jez. polsk. cynowod, polonais de la Prusse, Treichel, Poln. Vulgäm. jadić, lulica, pakujac, trolistnjak, ašibičar, sr'menik, orlica, orliček, ı'orlica, grlicel, koloticel, serbo-croate, ŠuLEK. orlylii (= petits aigles), ohsamit (= velours), dzvonotchliy (= petites clochettes), petit russien, comm. par M. TH. VoLKov. yoloublii (= petites colombes, russe, Schmalial'sen. [Th. V.]. liatilelei, lithuanien, ЈАсовг, Litauische Pflanzenn. ohsolini, bikerishi, letton, Ulmans, Lett. Lex. haranguirig, magyar, Fusz, Trivialn.

akhilia, arabe syrien, Berggren, Guide, 1844. - arabe algérien, Florian Pharaox, Voc. ar. cupeli-cineghi, turc, Sestisi, Voyage dans la Grèce asiatique, 1789.

(1) De l'anglo-saxon culfre et du mot wort racine. "From the resemblance of its flowers to little heads of sucl birds feeding together. "Prion.

(2). From the resemblance of the spurs to chickens drinking.

(3) Mot forme par fausse étymologie populaire. 


\section{I'EONLA OFFICINALIS. (LINNÉ). - LA PIVOINE.}

\section{1. - NoMs.}

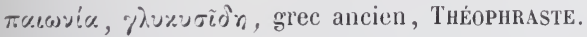

Tรv-ópobov (1) grec ancien, identification assurée par E. Fournier, Réf.

de la Nomencl. bot. (Journal des Savants, 1880; tir. à part, p. 9). glycysis, pxonion, aglaophotis $\left({ }^{2}\right)$, [selenion, selenogonon, dichomenion, menion, monogenion (3), theodonion (4), panticeras, grec,

APulÉE (Ve s. apr. J.-C.), De medicaniinibus, édit. Ackermann, 1788. uixos, grec mod., Somavera, Tesoro, 1709 ; SibThoRP, Fl. gr. prodr.

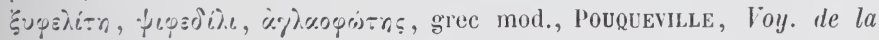
Gréce.

ซоขร์:ะ๐, grec mod. de Céphalonie, Hecdreich, Flore de Céph., 1883. pæonia, latiı. glyciside, pentorobon, latin, Puse, H. nat. glycisida nigra, pæonia græcorum, latin, Scribonius LARgus ( $1^{\mathrm{cr}} \mathrm{s}$. apr. J.-G), cité par Meier, Gesch. d. Bot. dactylosa, latin, Isidore de SÉVille (fin du VIe s. apr. J.-C.) rosa fatuina, latin, Apulée ( ${ }^{\text {e }}$ s. apr. J.-C.), De medicam. plionia, latin du Moyen-Age, Sainte-Hildegarde, selon Langkavel, Zur Gesch. d. Bot. aus Du Cange gr. Gl., 1866. glicida, pionya, latin du Moyen-Age, Mowat, Alphita. dactilos, glicida, dulcisiolı, latin du Moyen-Age, Diefenbacih, Gl. med. latin.

rosa asinina, oculus turimus, anc. nomencl., Continens Rhasis, 1486. rosa asinorum, neremisch, anc. nomencl., DE Bosco, Luminare majus, $1496, \mathrm{f}^{\circ} 29$.

rosa sancta, sancta rosa, rosa asimina, rosa benedicta, sancti Georgii

(1) Cette plante est ainsi appelée parce qu'elle a cinq carpelles dont chacune représente la gousse d'une légumineuse telle que l'öpobov. (E. Fournier).

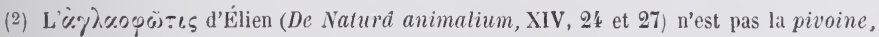
mais la mandragore. - Les noms qui suivent et que j'ai mis entre parenthèses sont ceux de la mandragore. Lerreur vient probablement de ce que le mot ìyluopöstıs en grec ancien a dû avoir en même temps le sens de pivoine et celui de mandragore. (En grec

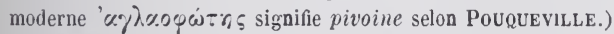

(3) Mauvaise lecture, sans doute, pour menogenion.

(i) Mauvaise lecture, pour theogonion? 
rosa, flos pæoniæ, anc. nomencl., C. Bauhinus, De plantis a sanctis nomen habentibus. Basileae, 1591.

aliephos, anc. nomencl., Srworrma (dans Mesues, Compendium, 1623.

aclaophotis Aeliani (1), pronia communis, pronia fomina, anc. nomencl., Bauhin, Pinax, 1671.

rosa regiu, anc. nomencl, Rosenthal, Symopsis plunt. diaph.

herba yicht, lat. du XIIe siècle, d’après des gloses latines-germaniques,

Descenet, Santa Ildeyarda.

peonie, f., anc. frans., Valdambert, Manière de nourrir les enfunts, 1565, 1. 315.

péone, f., anc. frans., J. Victon, Tesoro, 1709; Cotgrave, Fr. dict., 1650;

DuEz, Dict. fr.-it., 1678.

peoine, f., péoyne, f., anc. fr., L'arbolayre, 1490; Le grand herbier en

francoys (vers 1500); Canus, L'op. sal. (XVe s.).

pèione, piione, f., piione, f., wallon, Grandg.

pioniu, f., languedoc. du XIV s., Romania, 1883, p. 101.

fiône, f., normand, - picard, - wallon, - champenois, - lorrain. -

Haut-Maine. - départ. du Nord.

pionne, f., Doubs, Beavquer, V'ocab. - Vosges, Halllant. - Saint - Pol

(Pas-de-Calais), comm. par II. Eı. Eonont. - ancien wallon, J. Cinus,

Un Manuscrit namurois.

piyône, f., wallon, Feller.

pioine, f., anc. fr., P. MEYER, Deurième rupport s. une mission litt.

(Archives des missions, 1867

piouno, Var, Anic, Cons. s. Brignolles. - Gard, c. par M. P. Fesquet.

pioune, f., Pays-Messin. - wallon.

pionnier, m. (la plante qui donne les pionnes), ane. fr., Van Hasselt,

Ess. s. l'hist. de la poésie franc. en Bely. 1838, p. 185.

peroisne, f., peroine, f., anc. fr., J. Du Chesse, Tetrade des mululies du cervequ, 1625, p. 999; Сотgrave, Fr. dict, 1650.

pevoesne, f., anc. fr., Cotgrave, Fr. dict., 1650.

piroéne, anc. fr., Ch. Estienne, Dict. latino-gall., 1561.

piroine, f., pivoine femelle, f., français.

pirouéna, $\mathrm{f}$, canton du Vigan (Gard), Rovger, Topogr. du cant. du ligan. pirvini, Var, Hanre, Catul. - Forealquier, c. par II. E. Plauchud.

piboino, f., Castres, Covzinié, Dict. - Lot-et-Gar., c. par M. H. de Blaviel. pibouèno, f., Gers, Céxac-Ioncaut. - languedocien, Duboul. - Lot-et-

Gar., comm. par II. L. DARDY.

(1) C'est une erreur. L'aglaophotis d'Elien est la mandragore. 
pon, m., Les Fourgs (Doubs), Tissot, Patois des F.

ponpon, m., canton d'Arbois (Jura), comm. par II. H. DorboN.

piöme, f., Guernesey, MÉtivier, Dict. franco-nor'm. - Vosges, Halllant, Fl. $p$.

piaôme, f., Val de Saire (Manche), Rompanl, Pat. du Val de Saire, 1881.

péaume f., anc. f., Duez, Dict. fr. ital., 1678.

piorme, f., Bessin, Joret, Fl. pop.

piole, f., Manche, Fleuri, Essai sur le pat. norm.; Jonet, Fl. pop. Mazeley Vosges), HalllaNt, Fl. $p$.

plionme, f., Vendée, Lalanine, Gloss., 1867.

pienne, f., anc. fr., Cotgrave, Fr. dict., 1650.

rose de pienne, f., anc. fr., Ch. Estmexse, Dict. latino-yall., 1501; Cotgrave,

French. dict., 16.0).

rosse pienne, f. Somme, Ledieu.

rose pionne, f., Boulonnais, comm. par $\mathrm{II}$. E. Deseille.

arriso piboueno, f., Ouest du Lot-et-Gar., c. par. M. L. DARor.

roso, f., Briançonnais, comm. par .1. J. А. Сhamavı.

roso d'ase, f., Gard, Pouzocz, Fl. du Gard. - Forcalquier, (Basses-Ilpes),

c. par M. E. Placchud.

rose du diable, f., Izy Loiret, Rouvray-Saint-Denis Eure-et-Loir , c. par

II. J. POQUET.

rose du djuau, f., rise du mer, f., Spa, LezAdck, Noms wallons.

rose de Nostre-Dame. f., anc. fr., Ch. Estiense, Dict., 1561; L. Fucis,

Ilist. des pl. en commentaires, 1558; Cotgrave, Fr. dict., 16.50.

herbe de Saint-Jehan, f., anc. fr., Canus, Live d'heures.

touße, f., Haut-Maine. Montesson, Vocub., 1859.).

boule de feut', f., Pamproux Deux-Sèvres', comm. par M. B. Soucié.

boule de feu, f., Département de la Viemne, Labinne, Giloss., 1867.

llamponis, llampudul, Pyrénées-Orient., Cospanso, Hist. nat.

ebutiscle, Pyrén.-Orient., Ines.

hllor au malet (= fleur aux convulsions, suisse rom,, Bridel, Gloss.

flor de malet, f., erba de malet, f., Suisse rom., Vicat, Pluntes vénéneuses de la Suisse, 1776.

couviras, pl., Ban-rle-la-Roche Lorraine), H. G. OBeris, Description, 1806. parot, m., Laas (Loiret), comm. par M. L. BEalvillard. - Ascoux Loiret), comm. par M. L. MAtox. - Rouvray-Saiut-Denis (Eure-et-Loir), c. par H. J. POQuet.

yros parot, m., ergots de coq, m. pl. (Ies ovaires après la chûte des pétales', Rouvray-Saint-Denis Eure-et-Loir), c. par II. J. POQuet. parot de jardin, m., Ineuil Cher), rec. pers. 
flou de pabot, f., Luchon (Pyrénées), Jul. SACAzE, - Sud-0uest du Langued., Duboul.

grando pimpanelo, f., languedocien, SAurages, Dict. lungued. - Gatd, comm. par II. P. FEsQLeT.

penolho, f., arrondt de Saint-Pons (Hérault), Barruès, Gloss. bot.

bouffons, pl., Normandie (Roumois et Lieuvin), Joret, Fl. pop).

ivrogni, f., patois des environs de Lyon, l'uitspelt, Dict. du pat. lyonn.

ivogne, f., Lỵon, IDEM.

peonia, rosa de' monti, ital.

peonese, ital., DuEz, Dis. it. fr., 1678.

erbe casta, ital., Florio, New world of words, 1615.

amponia, folayne, Vérone, Pollins, Fl. l'er.

pionnia, Gènes, Casaccia, Diz. gen.

pionica, sicilien, Cupani, IIortus cathol., 1676; Lagusi, Erbuario, 1742;

MorTillaro.

peorica, Sardaigne du sud, Moris.

pioina, sarde, Spaxo.

peonia, sarde logodourien, SPAxo.

peonie sultaleghe, roese, Brescia, ZERsI.

peogna, ronagnol, MorRI.

pedogna, env. de Venise. Von Martens, IJ, 92.

franca de sirulia, Sardaigne du nord, Moris.

peonia, rosa albardera, yerba de Santa Rosa, rosa montesina, rosa del monte, espagnol.

rosa alubardera, province de Badajoz, Bibliotheca de las trad. pop. esp.

$\mathrm{Vi}, \mathrm{25}$.

rosa montesca, catalan, Lacaialleria, Gazophyl. catal. latinum, 1696.

peonia, rosa albarleira. portugais, BRotero, Fl. lusit.

pionia, galicien, Cuverro ; Valladares.

bujor, roumain, Cinac, Dict. daco-r. - roumain de Transylv., JarNik,

Doine, 1885 (supplém. gloss., p. 26).

beonien, benonien, beoni, beonienkraut, benignenkraut, pinell, pyonie, gichtrose, freissumrose, anc. allemand, Diеfenвасн, Gloss. med. lat. peonienros, benignenros, bergros, allemand, Rosenberg, Rhodologia, 1631, p. 171 .

minnewr: anc. h. all., Descenet, Santa Ildeyarda.

sant Jörgen blumen, allemand, C. Bauhinus, De plantis a sanctis nomen habentibus (1591).

viltman, anc. h. all., Germania, 1888, p. 308.

paschteblum, peischtross, Luxembourg, J. WEBER.

gerjerühs, allem. de Transylvanie, Fusz, Trivialnamen. 
chercharosa, pfaffarosa, gichtrosa, flätterrosa, heerabluama, brandrosa, canton de Saint-Gall, Wartmaxin, Volkshot.

bubenrose, kinderblume, kindliwehllume, Suisse allemande, Durheim, Schw. Id.

barillen, barillenrose, canton de Berne, IDEM.

pfingstrose, päonie, königsblume, künisgsrose, gichlkörner, mimwenkraut, amachtsblume, allemand, NEмNich.

kuhrose, allemand, Grimu, Wörterl.

benedilitrose, putennige, butthühnchen, butthenne, allemand, A. MüLLER, Syn. Namen, 1866.

antlas жr-rose, Lechrain, LeOPRECHTING, Aus dem Lechrain, 1855, p. 186. pegunje, pegonis, Oldenbourg, StrackerJax, Aberglaube und Sagen aus Oldenb.. 1867.

lutünge, Westphalie, LandoIs, Westf. Pflın:enn.

bigonnie, Prusse occid., Treichel.

hijoin, pijon, Prusse, Frischbier, Preuss. Wört.

bijenga, Dantzig, IDEM.

matönje, f., kaurôse, Goltinğue, Sснамвасн.

Aädam un Evi, hintje un hentje, Frise orientale, Focke, Pflanzennamen der unteren Weser.

buttoniros'n, puml. l'us'n, Basse-Autriche, F. HöFER, Nieder-österr. Pflanzennamen.

knopfrosen, Carinthie, Zwaxziger.

bobl, Bolımerwald, Schueller, Bayer. Wirrt.

kirchenrose, Eifel, Wirtgen, Vegetation der Eifel.

tisel (die Samenkapseln der Freonien) (langage enfantin), Silésie, Weinнlo Beitr. *u einem schlesisch. Wört.

pioen, pioenroos, boeremroos, stinkbloem, holland. et flamand, VAN DALE. [A. DE COCK].

sinksenroos, sinte Cornelisroos, flamand. [A. DE Cock]. hearbe pionie, anglais, Flonio, World of words, 1611. peony, piony, kingsblonm, rose of the mount, knightsbloom, anglais, Cotgrave, $\mathrm{Fr}_{r}$. dict., 1650 .

piny, West Somerset, ElWorthr, Word-book.

piney, île de Wight, LoNG, Wight dial.

pranet, Shropshire, Jacksox, Word-book.

nanpie, Yorkshire, Britten, Dict.

blodau 'r brenin (= fleurs du loi), gallois, J. DAviEs, 1632. H. G.].

coronllys (= herbe de la couronne), daearllys (= plante de la terre), rhosyn y mynydd (rose de la montagne), gallois, Hugh Davies. [H. G.]. lus-phoine, irlandais, O'Reilly. [H. G.]. 
lus pronailh, irlandais, FoLEr. [H. G.]. mackinnassayh, irlandais, J. KEOGH, 1735. [H. G.]. piuoena, breton, Tomenclator. [E. E.]. pivoëna, breton, P. Grégolre. [E. E.]. krycae yrt, péon, suédois dialect., Jexisex-Tusch, Tord. pluntenurne. pionrose, pintserose, gigtrose, liongerose, bonderose, hulsbåmlshaerner. danois dialect., IDEM.

marin koren, russe, Filk, Kenntn. d. russ. Reichs.

pionnaja rosa, russe, Nemsich, Catholicon.

vione, russe, Scimalhausen. [T. Ү.].

vivonia, petit russien. [T. V.]

piu'onlia, tchèque.

piwoniu, polonais.

bion, polonais de la Prusse, Treichel, Poln. Vulyärn.

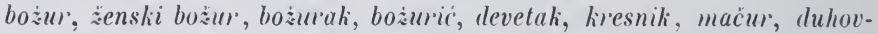
ska rusa, liraljev criet, lraljev crit, turkarica, kurjak, papinja, putonka, potonka pientia, trojaçke rośe, trojaskia rozicu, serbo-croate, Šulek, Jug. im. bilju.

piwunia, lithuanien, Mextzel, Leric. polygl.

slulpune, letton, Llaax, Lett. Lexicon.

leelas rohses, letton, STExDER, Lettisches Lexicon.

pénouna, yadjuard = rose vaillante), ariljuarl = rose d'ours), drapcotrouk = qui casse les pots par l'extension des racines', harsnouk. = petite épousée, une piroine de la petite espéce), harsin dzaghik une pivoine de la petite espèce , arménien, Alushas. [Er. L.].

ros:a-ba:sul, magyar, Ciнаc, Dict. daco-rom.

bus:aro:su, magrar, Miklosich, Slarische Elem. im mag., p. 64.

uard el himar, arabe, Continens Rhasis, 1486.

vuard el hhamir (= rose des ànes), anc. arabe d'Espagne, Iвх EL BeїтнAR,

trad. Leclerc, III, 108. - arabe algérien, Leclerc, Trad. d'IBx EL B. feonia, arabe, SÉRAPION L'AXCIEN.

fuouniu, arabe syrien, Berggren, Guide, I814.

fïouiniu, liehyina, arabe, IBN EL BEїTHAR.

cheqaïq, arabe algérien, BeL Kassem, Dialogues, 1877.

a'ouid ec calib (= bois de la croix), arabe, IBN EL Beïthar, III, 408. arabe algérien, LECLERc, Révélations des énigmes, 1874.

yul chatiaili, turc.

ayou qoulaghi (âyou $=$ ours), turc, Mesinski.

tschigenak, tatare, Falk, Kenntn. d. russ. Reichs.

tschunuk, kalmouk, IDE.M.

tscheina, téleute, IDFM. 
karinz, langue inconnue du XIIe siècle (Pays de Wiesbade), Descemet, Santa Ildegarda.

\section{II. - Locutions et PROYerbes.}

1. "Être rouge comme une piroine." - Se dit de quelqu'un dont le visage est enflammé (Locution française).

«Pivoiner = rougir, $»$ français.

\section{Fr. Michel, Dict, d'argot.}

( ..... Quel était l'homme? - Un Normand, figure ronde, pivoine.....» Louis Norr, La belle Marinière, roman.

( Er glüht wie eine Fijon (ist betrunken). »

Prusse orientale, Treichel, Poln. Vulgaemamen.

Un Briggand très populaire en Moldavie, Boujor, a reçu ce nom qui signifie piroine, à cause de la couleur de ses cheveux.

\section{Alexaxdi, Ballades... de la Roumanie, 185..}

2. «La belle pivoine ne sert guère qu’à récréer la vue; la fleur du jujubier quoique petite, donne un bon fruit.

Proverbe chinois, DoolitTle, Vocab. of the chinese language, 1872.

3. Apertu comu la pionica, si suol dire di cosa sconciamente larga, e maltrattata dall'uso, o da altro accidente.

Sicile, Mortillaro.

Farisi, o divintari na pionica, dicesi di cio, che perde il suo primo essere, e l'antica figura, e deteriora assai.

Sicile, Nortillaro.

\section{FOLKLORE.}

« lirtutes paeoniae ad ischiudicos. Herbae paeoniae radicis partem alligabis lino, eumque, qui patitur, circumcinges, res est saluberrima Et si eam in navi habueris, tempestatem compescit, si mundus, ea utaris, est enim mirabilis. 》

Apuleius (Ve s. ap. J.-G.) De medicaminibus. Edit. Ackermann, 1788, p. 229.

«Herba paeonia si lunatico jacenti ligetur in collo, statim se levat sanus. Et si eam secum portaverit, nunquam ei hoc malum accidet. »

IDEM, Idem.

Si l'on fait avec les fruits de la pivoine un collier et qu'on le fasse porter par un enfant épileptique, il guérit.

ET-TerNiNy, auteur arabe cité par IBN-Beïthar, trad. LeCLerc, III, p. 16. 
Dans le pays de Liège le peuple fait avec les semences de la pivoine (peus di Saint Gérô, pois de saint Gérard) des colliers de dentition destinés a prévenir les convulsions et à faciliter la ponsse des dents. On en prend 32 graines qu'on fait tremper 24 lieurcs dans l'eau béuite, puis on les enfile sur de la soie rouge, au moyen d'une aiguille n’ayant jamais servi.

SEMERTIER.

L'espèce de piroine employée contre l'épilepsie est surtout la femelle. On prétend que, si on la coupe avec du fer, elle perd cette propriété.

EL-GHAFEKY, auteur arabe cité par IBN-BEïthaR, III, p. 16.

Sa graine ou sa racine, cueillie au défaut de lune, pendue au col et appliquée sur les poignets ou seule avec guy de chesne, est préservatif singulier contre le mal de Saint-Jean (épilepsie).

XVI siècle, La Maison rustique, 1'e édition.

«Pour préserver les enfants des conchussions (convulsions), on leur fait porter un collier de graines de pivoine, qu'on appelle collier de conclussions. » Loiret, communication de M. L. MaLon.

Pour guérir l'épilepsie on attache au cou du malade un collier composé de 40 graines de piroine. Chaque jour il doit en détacher une graine, la piler, la mettre dans de l'eau en y ajoutant trois gouttes de sang de son père et antant le sa mère et boire le tout.

Danemark, Kristensen, Folkeminder, vl, 380, 256 [H. F. F.].

Pour favoriser la dentition on fait porter aux enfants des chapelets de graines de pivoine.

Jutland (Danemark), c. par M. H. F. Feilberg.

« Io pescoco dos meninos os preserva de gotta coral (épilepsie). 》 Portugal, Revista scientifica, Porto, 1883, I, $5 \underline{2} 3$.

La pivoine a de grandes vertus contre les démons et les maladies. La Belle Mère de Moyse, tourmentée par les démons, alla, sur le conseil de Moyse, sur la montagne, et là, Dieu lui fit connaître les vertus de cette plante et il la guérit. - Arménie, Alsshax [Er. L.].

Pour déraciner la pivoine, (1) un prêtre va avec la croix et l'évangile auprès d'elle, en lui disant: « je te salne, herbe. » P’uis il lit sur elle le cinquième psaume en disant: «Dieu, sois béni, car à cause de l’innocent Mö̈se, tı as fait de cette plante un médicament pour toutes les maladies. Nous te prions de lonner la force à cette plante contre les maladies et les démons. 》

Tralition arménienne, Alisian [Er.-L.].

(1) Il faut que la plante soit déracinée et non coupée, pour qu'elle ait toute sa vartu. 
Cette plante est un préservatif contre les illusions nocturnes causées par les faunes (cauchemar). On recommande de l'arracher pendant la nuit, parce que si l'on est aperçu par le pivert il atlaque aux yeux, pour la défendre, celui qui la cueille.

Pline, Hist. nat., XXV, (Édit. Littré).

La pivoine, mise sous l'oreiller pendant la nuit, vous fait avoir des rêves charmants. - Donnée à un nouveau né, avant qu'il ait commencé à téter, elle l'empêchera de devenir méchant par la suite. Gardée à la maison elle empêche les serpents et les animaux féroces de s'en approcher.

Armenie, Alishan. [Er. L.].

Peonienrose vertreibt den Alp.

Tyrol, I V. ZiNgerLe, Einige Notizen aus einen Kraeuterb.

(Dans Zeitsch. f. d. d. Wyth. IV).

Wer zur See unter Sturm zu leiden hat, entzündet diese Planze, um den Sturm besänftigen. - Legt man die Pflanze auf den Mondsüchtigen, wenn er liegt, so wird er bald gesungen.

Superstition anglo-saxonne, Fiscner, Aberglaube unter den Angel-Sachsen (dans Programin d. Gymn., lleiningen, 1891 p. 31).

Si une jeune fille absorbe de la pivoine pendant 12 jours de suite, elle aura les joues aussi rouges et aussi fraîches que cette fleur.

Arménie, Alisilav. [Er. L.].

On met une piroine dans le premier bain d'un nouveau né afin qu'il soit toujours frais et rose comme cette plante.

Roumanie, c. par II. A. GOROVEI.

Dans certains villages de la Flandre on croit que les feuilles de pivoine, mises dans les souliers ou les bas, empèchent la conception; aussi les jeunes filles, allant aux kermesses arec des amoureux entreprenants, n'oublient-elles jamais de s'en pourvoir - s'il y a moyen.

A. DE Соск, Volksgeneeshunde in Vlaanderen, Gent, 1891, p. 51.

P.EONT.1 CORALLINA. (RetziUs.).

inyouvic, île de Zante, Margot, Flore.

paeonia mas, anc. nomencl.

pivoine maile, f., pivoine corail, f., français.

pampalonia, f. Minorque, Costa, Flora. 


\section{PEONIA RLSSI. (BroNA).}

rouman el chad (= grenade du singe), arabe. Haxotesu, La Kabylie, 1872 .

tharoumant guiddaoun, kabỵle, IDEM.

\section{PAEONZA PEREGRINA. (MiLLei).}

Чै५ $1553, \mathrm{p}, 18$.

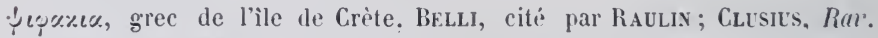
plant. hist. 1601.

paeonia alba. nomenel. de Cussius.

\section{P.EONI.1 MOUTAN. (SIMS.).}

piroine ell arbre, f., piroine moutan, f., moutan, m. français.

mou-tane (= pivoine arbrisseau), mou-cho-yo (= pivoine arbre), haoouk (= roi des fleurs), chinois.

botun, japonais, HoFrmax, Noms indigèues des plantes du Japon, 1864.

Cette plante, originaire de la Chine, a été introduite en Angleterre en 1794, en France en 1807, où l'Impératrice Joséphine la fit cultiver pour la première fois à Malmaison.

Bon jardinier pour 1811 , p. 655 .

Selon le dictionnaire de D'Orbigny elle a été introduite en Angleterre, par Jos. Banks, en 1789, et en France en 1803.

Cette plante a été le tout temps cultivée arec passion en Chine. Voyez détails intéressants à ce sujet dans Grosier, De la Chine, 1819, t. III, p. 24-40.

Lne variété de cette pivoine porte le nom de piroine rose, piroine en arbre odorante (selon D'Orbigny, Dictionnaire); piroine à odeur de rose (selon le Bon jurdinier pour 1843, p. 858 .)

CI.MICIFUGA F(ETIDA. (LINÉ).

\%ó,co\%, grec, IsIDORE DE SÉville fin du VIe s. apr. J.-C. Identification faite par Merer, Gesch. d. Bot.).

cimicia, latin, IDEM. 
actæa cimicifuga, thalictroïdes fætidissimum christophorianæ facie, anc. nomencl., Neunich, Cath.

chasse punaise, français ( $\left.{ }^{1}\right)$ savant, IDEM.

cimicaria, italien, IDEM.

stinkendes wamzenliraut, allemand savant.

ploshnitcare $\left({ }^{2}\right.$, roumain, Ciнаc, Dict. daco-rom.

plustiewnil, (= plante aux punaises), polonais, IDEu.

vonioutchlia (= la puante), petit russien, c. par M. TH. VoLkov.

\section{AC'T.E.A SI'ICATA. (LINXÉ). - L'HERBE DE SAINT-CHRISTOPHE.}

actrea, latin, PLine, Hist. nat.

herba Christofori, lat. du moyen àge, Bartholomeus, Sinonoma, cité par Mowat.

herba sancti Christofori, oculus Christi, DE Bosco, Luminare majus, 1.196, fo $\left\{\begin{array}{l}\text {, } \\ \text { recto. }\end{array}\right.$

aconitum racemosum, napellus racemosus, christoforiana, costus niger. barlua cupri, anc. nomenclat., Bathix, Pinax, 1671.

helleborus niger falsus, anc. nom., Bosenthal, Synopsis.

erba de sant Christofol, Pyr.-Orient., Comparyo, Hist. nat.

erbo de san Christoou, f., Gard, comm. par H. P. FEsQUet.

herbe Saint-Christophe, f., herbe de Saint-Clwistophe, f., français.

christophoriane, f., Meuse, Labocrasse, Gloss.

christophorine, f. Lyonnais, CARIot, Etude des fleurs. [ED. EDM.].

hellébore noir, m., centre de la France, La lléridienne de l'observatoire,

Paris, 17i1, p. 61.

varaïré negré, m., Gard, Pouzolz, Flore.

raisin ou lau (= raisin du loup), m., Châteaı d'Vex (Suisse), Vıcat,

Plantes vénéneuses de la Suisse.

actée en épi, f., français savant.

barba di capro, italien, TARGioxi, Diw.

erba San Cristofolo, Venise, Boerio, local. renez.

erba San Ciristofen, milanais, Banfi, Voc. mil.

erba d' San Cristöfo, cristöforiana, piémontais, ZaLl, Diz. piem.

tarfojela, Parme, Malaspisa, Voc. parm.

(1) Le mot n'est pas d'origine populaire, car la plante n'existe pas en France.

(2) Du mot ploshnitsa qui signifie punaise. 
yerba de San Cristobal, cristoforiana, espagnol.

erba de San Cristófol, catalan, Varreda, Catalech.

christoforitca, ierba Sf. Cristofor, ierba talharului, roumain, BRANDzA,

Limba bot.

hristofóare, roumain, Cinac, Dict. daco-rom.

jarbe Christoforului, roumain de Transylranie, Fusz, Trivialu.

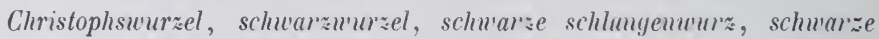
nieswurz, falsche niesu'urz, allemand.

muderkraut, mulerliêr, yeftschwms, nolefshraut, Luxembourg, J. WeBER. visselnkrüt, n., heilensch w'undkrit, Gotlingूue, 今снамвасн.

wuhlefsururzel, allem. de Transvlvanie, Fesz, Trivialu.

wolfswurzen, berufkraut, Suisse, PriTz. et JEss., Volksn.

hexenkraut, Suisse, Derueim, Schw. ldiot.

flächachrut, cant. de Saint-Gall, Wantmaxn.

johanneskraut, sunauemlliruut, Basse-Autriche, F. HöFER.

mutterbeeren les fruits), Eifel, Wintges, Veg. der Eifel.

sinte Christoffels-cruylt, anc. flam., DodoxaEus, Cruydt-boeck. [A.DE C.]. Kristoffelliruid, hollandais.

bane berries, anglais, Nexnich, Cathol.

llys cristoffis (= herbe de Saint-Christophe, gallois, J. DAviEs, 1632.

[H. G. .

euor $d u=$ hellébore noir, breton, Nomenclator. [E. E.].

padlbär, trollbär, trolldrufua, villdrufia, humlbär, getelortar, suédois

dialectal, Jexisex-Tusch, Noril. pl.

troldbär, svinebiir, soulórt, norvégien, IDEM.

Christophersurt, druemunke, gallebär (les fruits), danois dialect., IDEM. habulica, vuiji koren (= racine des loups), serbo-croate, Šulek, Jug. $i . b$. worones, christoforowa trawa, soroko-pritotshlia, russe, Neмnich, Cath. roronii iagolly (= fruits des corbeaux', russe, Schмalhausex. [Th. V.].

tchernet: (= moine), vortchi iahorly $:=$ fruits du loup), petit russien, comm. par M. Th. Yolkor.

samorostlik (= croissant de lui mème), tclıèque, Šulek, Jug. $i . b$. farkas szölö, magyar, Fusz, Trivialn.

sammakion yrti, sammation marja, finnois, Fellyax, Index pl. siuvo marjad, akkilise haiguse rohhi, esthonien, IDEM. 


\section{A GNOLI A GEES}

\section{.MAGNOLIA (Genre). (LINNÉ). - LE MAGNOLIA.}

maynolia, m., maynolier, m., français.

magnoria, m., Lot-et-Gar., c. par M. L. DARDY.

laurier-tulipier, m., français du XVIII siècle, DrhaMeL DU MoxceaU,

Traité des arbres qui se cultivent, 1755. t. II, p. 1. - Le mot lauvier-tulipier a été remplacé par le mot magnolia, seul usité aujourd'hui .

maynole, f. (la noix de l'arbre), français, LitTRé, Dict.

bois-pin, m., La Martinique, J. Turiault, Etude sur la lungue créole, p. 230. bois cachiman, m., La Guadeloupe, IDEM.

laurel tulipan, espagnol, CoLmeiro, Dicc. de los nombres.

flor de corazon, espagnol mexicain, Poret, Notices sur le Merique, 1863.

Pour faire honneur au botaniste français l'ierre Magnol, mort en 1715, on a donné son nom à cet arbre dont la culture a été introduite en France au XVIIIe siècle.

LIRIOINENIIBON TLLIPIFERA. (LINAE): - LE TLLII'IER.

liriodendrum, liriodendron, tulipifera, tulipifera virginiana, nomenclat. savante.

tulipier (1), m., tulipier de Virginie, m. français.

toulipiè, m., Languedoc, Duboul.

tulipe en arbre, f., français, FiLlassier, 1791.

tulipero, tulipanero, espagnol, CoLmeIro.

hickory poplar, cucumber-tree, magnolia, anglais des Etats-Unis, BERGEN.

(1) Ainsi nommé parce que ses fleurs ont une certaine ressemblance avec les tulipes. 


\section{A NONAGÉES}

\section{ANONA (Genre). LixNE. - LE GOROSSOLIER.}

corossolier ( $\left.{ }^{1}\right)$, in., français.

corossol, m. (le fruit), français.

corasol, m., cachimas, m., Intilles, Rochefort, Ilist. nat. des Antilles, 1665.

cachiman, m., français.

custarl apple, anglais colonial, Grisebaci, Fl. of the west-ind. isl.; Braxdis, Forest flora of Indiu.

Pour l'historique et les usages des espèces de ce genre, royez M. F. Dural, Monographie les Anonacées. Paris, in-4, 1817.

Linné a appelé ce genre anona du nom mexicain anon (espèce de corossolier). Pour lui donner une tournure classi(yue il a trans?ormé anona en annona. Les botanistes modernes ont rétabli anona.

\section{A.VONA MLTRICAT.A. (LINNÉ).}

corossolier épineur, m., corossol épineur, m., cuchiman épineux, m., français.

corossol fruit, La Guadeloupe, L'Herminer, Synonymique créole des arbres, 1834.

corossol hérissé, m., sappadille, f. (le fruit), français, Correrchel. p. 332. sour sop, angl. des Antilles. West, 1794.

\section{ANON.1 CHERIJOLI.1. (MILL.). - LE GHÉRIMOLIER.}

chérimolia, m., chérimolier, m., français.

chérimoya, Guadeloupe, L'Herminer, Synonymique.

cherimolia, anglais colonial, GrisEBACH, Fl. of the west ind. islands.

(1) Cet arbre tire son nom d'une isle hollandaise nommée Curaço d'où il a êté porté dans nos colonies.

Nicolson, Ess, sur l'hist. nat. de Saint-Domingue, 1776. 


\section{ANOA.1 RETICLLATA. (LINNÉ). - L'ASSIMINIER.}

anona triloba, nomenclat. savante

assiminier, m., français, FILlassier, 1791.

creur de breuf, m., pomme cannelle, f., Guadeloupe, L'Herminier, Synon.

manillier, in., assiminier de Virginie, m., français, Couverchel, p. 332.

anona de C'uba, catuche de las Antillas, espagnol, CoLseiro, Dicc.

custard apple, angl. des Intilles, West, 1794.

sour sop, anglais colonial, Grisebich, Flora.

bullocli's lieart, anglais de l'Inde, A. DE CAnboLLe, Orig. des pl. cult., p. 138. yrislımadja, lavani, attrpya, gandagritra (le fruit), sanscrit, BoEнтLingK.

ANONA I'ALISTTIS. (LINAÉ) ET ANONA MYCOS.1. (JACO).

cachiman morreux, m., Guadeloupe, L'Hermixier, Synonymique créole. cachiment marron, m., Saiıt-Domingue, Nicolson, Ilist. nat. de SaintDomingue, 1776.

monkey apple, alligator apple, corkwood, anglais colonial, GusEвıCH, Flora of west ind. islands.

\section{ANONA P'ALLIOOSA. (ALBLET).}

mamin, Guadeloupe, L'Hermixier, Synomymique créole. momin, Antilles, Rochefort, Ilist. nat. des Ant., 166j. doy apple, angl. des Antilles, WEst, 177i.

\section{A.YO.Y. SQQL.1.MOS.L. (LINAE). - LATTIER}

pomme canelle, (le fruit), f., français.

cachimentier. m., pommier de cannelle, m., français des Antilles,

Nicolson, Ilist. nat. de Saint-Domingue.

cachiment le fruit), IbE.

cachimas franc, m., Intilles, RocheEort, Hist. nat., 1665.

attier, m., atocire, français, D'()RBIGNY.

atte, f., (le fruit), français, Couverches.

ata, pinlıe, portugais brésilien.

atas, atocira, portıgais, Comizus, 1696.

sweet sop, anglais colonial, Grisebach, Filora.

custard apple, anglais de l'Inde, Gayble, Munual of indian timbers, 1881. sugar apple, angl. des Intilles, WEst, 1794. 
siti, sanscrit, Watson, Names of indian plants, 1868.

sitaphal, sharifa, hindoustani, Gamble, Manual.

sitapundu, télougou, IDE.u.

attî, Farroukhabad, Fallox.

qechtah $\left({ }^{1}\right)$, arabe égyptien, DELILE, Fl. xgypt. illustr.

khesta, arabe égyptien, CLot-Bey, Aperçu sur l'Egypte.

dzouzet er reqiqa (= Anona athiopica), arabe algérien commercial, LECLERC, Rérélations des énigmes, 187.1.

ahate $\left({ }^{2}\right)$ de pamucho, mexicain, Hernandez, cité par A. DE Candolle, Orig. des pl. cult., p. 136.

M. A. De Gaxdolle, Origine des plantes cultirées, 1883, p. 133 et suiv., prouve que l'Anona squamosa est originaire d'Amérique, d'où elle a été transportée dans les autres parties du monde. Il discute sur les noms mexicains et indous de cette plante, et sur l'étymologie de anona.

ROLLINIA SIERERI. (A. uE Gandolle).

sugar apple, anglais colonial, GRISEBACH, Flora.

YYLOPIA (Gemle). (LINNÉ).

african pepper, bitter-wood, anglais colon., GRISEBACH.

$$
\text { OXANDRA (Geme). - (Rich.). }
$$

lancewood, anglais colonial, GrISEBACH, Flor $\ell$.

$$
\text { CANANG.L (3) (Geme). - (Rumpinus.). }
$$

anona odoratissima, nomencl. savante.

canang aromatique, poivre d'Ethiopie, français, Couverchel.

lancewood, anglais colonial, GriseBACH, Flora.

(1) Le mot signifie lait coagulé.

(2) D'où le français atte, l'hindoustani atta, le portugais ata.

(3) C'est de l'espèce appelée cananga odorata (Hook) que l'on extrait un parfum célèbre en Orient, l'alanguilan de la Chine. 


\section{MENISPERMÉES}

\section{MENISPERMLM COGCULUS. (LINNE). - LA GOQUE DU LEVANT.}

\section{1. - Noms DE CETTE PLANTE.}

cocculae officinarum, cocculae oriontales, coco orientalis, cocci orientales, baccae orientales, baccae piscatorix, cucculus indicus, ancienne nomenclature, BauHrs, Pinax, 1671.

anamirta cocculus, nomenclat. de Wight et ARxotr.

cocculus suberosus, nomenclat. de DE CANDoLle.

menispermum lacunosum, nomenclat. de LAMARCK.

tuba baccifera, nomenclat. de Rusphius.

coque du levant, f., français.

coque levant, coquelerain, wallon, Severtier.

couvico, languedocien, c. par M. H. FAv.

émbéoutlo, f., Gers, c. par М. H. D.ignestous.

cóco, f., Corrèze, Béronie. - Lot-et-Gar., c. par M. L. DARdY. - Basses-

Alpes, c. par II. E. l'LAuchi'd.

coco de levanti, coccola di levante, ancien italien.

cócola, Brescia, Zerzi.

cócch, milanais, Cherubisi, Supplém. .

yaddaredda di livanti, sicilien, LAGusi, Erbuario, 1742.

galla di levante, italien.

baricocla d' levant, romagnol, MoRri.

coca de levante, coco de levante, espagnol, Oudin, Trésor, 1660.

coccas, coccacira, portugais.

kockellïrner, pl., kockelstïrner, pl., kokoshörner, pl., fischlörner, pl., liuckelstiörner, pl., levantische kokelkörner, allemand.

kockelefany, bavarois, ScHnELler.

feschkèren, m., kokelter, m., Luxembourg, J. Weber.

coculevant, allemand, Mexisski.

kolieltjes, hollandais.

water quaad, hollandais, Commelin, 1696. - Boulliy, Diss. s. la coque du levant.

cockles, anglais.

vaïr cherendat, arménien, ALISHAN. [ER. L.]. 
mihisehre, semm es semek (= poison du poisson), arabe, IBx el BeIthar, édit. Leclerc, II, g99; Freytag.

hhorit, tha'm es sumek, arabe, Dozr, Suppl. un.r dict. ar.

sikrian el-haont, arabe du Naghreb, Івх BeithaR.

baleq ote, ture, Barbier de Mexnard, Dict. tru'c.

silir fon̂yronghi (= cauda bovina), turc, Frestag.

2. - On se sert de la coque du levant pour enivrer le poisson dans les rivières et le prendre plus facilement. C'est ce qu'on appelle dans le midi de la France encoquer le poisson (encouca). On dit de queleu'un qui s'est enivié qu'il s'est encoqué.

RoLLand, Dictionn. des expressions vicieuses, Gap, 1810.

3. - Pour l'historique et les usages de cette plante voyez:

B. Connoxcm, Tractatus de baccis orientalibus idans son ouvage : De cluristiani et tuti medendi ratione, Ferrarix, 1591).

Boullay, Dissertut. sur l'hist. nat. de la Coque du Levant, $18 \mathrm{I8.}$

x. Waldich, Descript. of indian plants (dans: Asiatic researches, Calcutta, t. XIII $(1820)$, p. $403-112)$.

Flëckiger ANd Hanbury, Pharmacographia, p. 30-31.

\section{MENTSTER.II U GOIIIFOLIU.J. (LINAÉ).}

\section{1. - NOMS DE CETTE PLANTE :}

cocculus cordifolius, nomencl, de DE CaxdoLle.

tchinna, tchimnuriha, kundali, cukrapushpiki, madhuparnkit, madhuparni, somavalli, somalatiki, amṛti, amṛturalli, toyatant, toyavalliki, sudihurallikî, tchandri, tchandrahâsî, saçilékhi, tchakralakshanâ, bhishakpriyâ, surakṛtu, yadjnünyi, yadjnatalli, vadjrì, tantrikiti, indukali, nirdjara, guẹlutchi, tiktaparvan, vranahi, tchchadmitia, agniçikha, piṇtailn, udbhâri, djraraghna, rishagha (= Gift zerstörend), pittaghnı (Pitta = die Galle), vatsâdani ('), sanscrit, Bєнтlingk, Sanscr. Werterb.

umṛtavalli, yariụacélu, yarila, mahratte, MoLesworтн, A dictionary mmathee.

yulancha, hindoustani, Flëckiger, Pharmacogr., p. 32.

(1) Vatsidlana signifie proprement qui mange ses veau.x, ses petits; " so genannt weil die Pllanze nur eine oder zwei von drei Beeren zur Reife bringt. "BoEhtlixak. 


\section{CISSAMI'ELOS I'AREIRA. (LINXÉ).}

1. - Nons de CEtTe plante.

cocculus chondodendron, nomencl. de DE CANDoLle.

liane à gelée, f., liane à glacer l'eau, f., liune à tête de serpent, français, Nemnich, Lexicon. 1793.

liane ì cœur, f., Saint-Domingue, Nicolsox, IIist. nat. de Saint-Domingue. racine de Butua (la racine), français, Fü̈ckiger, Pharmacoyr., p. 25.

butua, espagnol, Nexich.

cipó de cobras. herea de Nostra Senhora, portugais, Moxterro, 1765.

velvet leaf, anglais des Antilles, WEST, 1794.

touvdruif, hollandais, NFMicH, Lexicon.

grieswur:el, allemand, NEnNich, Lexicon.

2. - Pour l'histurique, voyez :

Fuëckiger, l'hurmucographia, 1874, p. 9-7-27; Hewetus, Traité des maladies les plus fréquentes, 1773, p. 98.

3. - Para se saber se uma pessoa nos quer bem ou mal : Cortam-se as hastes da herra de Nossa Senhora, quando acaba de dar flòr, e pôem-se as escuras, por intençio d'essa pessôa. Łe nos quer bem, a lierva conserva-se verde; se nos quer mal, murcha.

Portugal, Revistu do Hinho, 1894, $\mathrm{n}^{\circ}$ i.

\section{BERBÉRIDÉES}

\section{BERBERIS IULGARIS. (LINAE). - L'ÉPINE-VINETTE.}

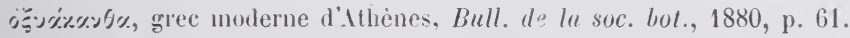
crespinus arbor, crispinum, berberi, amirberis Avicennae, darsisahan, latin du XVe siècle, DE Bosco, Luminure majus, 1496, fo 41, verso; fo 46 , recto.

oxiacantum, oxicuntum ( $\left.{ }^{1}\right)$, lat. du Moyen-Ige, MowAT, Alphita. berberis dumetorum, berberis, amirbaris Avicenno, spina acida, oxya-

(1) M. Mowat a lu, à tort: oriacantum, oricantum. 
cantha, oxyacantha Galeni, crespinus, anc. nomenclat., Bauniv,

Pinax, 1671.

crispinus, lat. du Moyen- $\Lambda$ ge, Diefenbach.

crispila, lat. du Moyen-Age, Germania, 1888. 1. 302.

barbarica, latin du Moyen-âge, PIPER, Altı. Pflanzennamen, 1881 ; Ger-mania, 1881, p. 402.

sancta spina, anc. nomenclat., BauHiv, De plantis a sanctis nomen habentilus, 1591.

barbari, m., anc. français, Ouds, Trésor des deux langues, 1660.Orléanais, comm. par M. L. Malon. - Ineuil (Cher), rec. pers.

berberin, m., anc. franc., Duchesse, De stirpibus, 154.

berbelin, m., Vosges, Haldant, Flore. - Pays messin, rec. personn.

barbelin, m., Vosges, Haldaxt, Fl. - Meuse, Labourasse, Glossaire.

barbeline, f., Romont (Vosges), Haildant, Flore.

burbeleire, Charmes (Vosges), Hallant, flore.

spinette rinette, f., anc. franc., Le grand herbier en francoys (ver's l'an 1500 ).

espine vinelte, f., anc. fr., Duchesse, De slirp., 1544.

espincte vinete, f., anc. fr., Camus, L'op. salern., p. 42, en note.

espino vineto, f., Var, Amic, Considérations sur Brignolles.

épine vinette, f., berberis, m., vineltier, m., français.

épine minette, f., Montbarrey (Jura), rec. pers.

mineto, f., Rouergue, Mistral, Tres.

pine vinette, f., Haute-Marne, comm. par M. A. Daguin. - Lyon, Molard,

Le mauvais langage, 1810. - Allier, c. par M. F. Duchon DE LA

JAROUSSE.

pilevinette, f., pilvinelte, f., Aube, DEs Etangs, Noms pop. 1844. --

Haute-Marne, comm. par M. I. Daguin - Fribourg, Grangier, Gl.

frib. - vaudois, Callet, Gl. vaud.

pulvinette, f., Bourberain Côte-d'Or), Raвiet, Pat. de B.

bisbinette, f., Ormoy-sur-Aube (Aube), Des Etangs, Noms pop. - Haute-

Marne, comm. par II. A. DAguin.

épinelle, f., Thévray Pays d'Ouche, Normandie), Joret, Fl.

vinette, f., anc. fr., J. Victon, Tesoro, 1609; Cotgrave, French dict., 1650.

vineta, f., Basses-Alpes, Annales des Basses-Alpes, III, p. 51.

vineto, f.. Gard, comm. par II. P. Fesquet. - provençal mod., Mistral rigneto, f., Périgord, Mistral, Tres.

bineto, fr., gascon, IDEN.

vinatier, m., vinettier, m., anc. fr., Cotgrave, Fr. dict. 1650.

épinevinier, m., crépinier m.. français, Barbeu-Dubourg, Le Botaniste francois, 1767. [Eo. EDs.]. 
vinetie, m., provençal moderne.

vingnetier, m., anc. fr., J. Camus, Lirre theures.

vinoté, m., environs de Saint-Jean d'Angély (Charente-Inférieure), Vinet,

Noms de quelques pluntes, 1864.

vinotier, m., Poitou, A. DE J'ssied dans Bull. histor. 1886, p. 283.

vinégreto, f., provens. mod., Liovs, Végétıux des B.-du-Rhơne.

vinaigrette, f., Haute-Marne, comm. par M. A. D.auin.

pisse vinaigre, Centre, BorEav. - Haute-Marne, comm. par M. A. Daguin.

- Villefranche (Rhône), comm. par M. DÉREsse. - Lyonnais, Puitspelu.

agradlelle, f., anc. franç., OGIER Ferrier, Remedles de peste, 1548, p. 35. ayradela, f., Basses-Alpes, Annales des Basses-Alpes, IlI, p. 51.

agradelo, f., provençal mod. - languedocieı.

aïgradelo, f., aïgreto, f., Gard, comm. par l'. l'Esquet.

agrivoutie, m., agrivoutat, m. (le fruit), Basses-Ilpes, Ammales des Basses-

Alpes, II, 280.

agrioutat, environs d'Arignon, PaLux, Calalogue.

eigret, m. (le fruit), provenç. mod., Mistral, Tres.

épine-aigrette, f. Lyommais, C.Mrot. [ED. En.].

oseille des hois, f., Hante-Marn', comm. par M. A. Dagun.

oseille du berger, f., Arcis-sur-Aube Aube), L'Arcisien pour 1868, (almanach).

pain de chève, m., Montbéliard (Doubs), Costestan, Glossaire. - Audincourt (Doubs), rec. pers.

pain de tchièrre, m., Montbéliarl, Coxteseax, Gloss.

tchivafoui, m., tsimafoui, Suisse romande, Bridel, Gloss.

tchivafou, m., tchivafoui, m., Suisse romandr, Vicat, Hist. des pluntes rénéneuses de la Suisse, 1776.

tsivra à fu, f., Vallorbes (Suisse rom.), Villotton-Albert, Vallorbes, 1875. tseirafu, m., canton de Fribourg, Helvetischer Almanach für 1810, p. 116. couonne de chieuffe, Moyenmoutier (Vosges), Haillant, Flore.

cavette, canton d'Aime (Savoie), comm. par M. Marjollet.

aube espene, f., Verviers (Belgique), LeJEune, Flore de Spa.

arbèspenne, arbèspine, arlispenne, arluspine, rotcl sipenne, wallon,

FELLER.

arlespenne. f., Verviers, Lfueune, Flore. - Spa, LezAack, Noms wallons. noblépéne, f., noliepéne, f., Valenciennes, Hécart.

espine benoiste, f., anc. fr., Cotgrave, Fr. dict., 1650.

épine-croix, fr., épi-croix, f., Haut-Maine, Ilontesson, Vocab

groseillier salivage, m., Châtenay (Seine-et-Marne), rec. pers.

arrégoulè, m., arrégolés, f. pl. (les fruits), Luchon (Pyrénées), J. SACAZE. 
nâre pique, Bru (Vosges), Halllant, Fl.

ekouanei, m., ekouennei, m., ékoina, f., Suisse romande, Bridel, Gloss. ékoana, f., Bas Valais, Gildieron, Pat. de Vtonnaz.

abagnourie, m., esvilhour, m., Alpes dauphinoises, MoutIER.

abaniouriè, m., (l'arbuste), abanior, m., (le fruit), esvelioour (1), m. (le

fruit), Briançonnais, comm. par M. A. Cirabrand.

rodzete, f. pl., (les fruits), lagnard (Suisse), Convu, Phonol. du B., 1877. bois de Saint-Girâ, m., Spa, Lezanck, Noms wall. - Liège, Forir, Dict. lwe d'Saint-Djèrî, m., wallon, Feller.

mévuron, m., Damprichard (Franche-Comté), Jém. de la soc. ling. VIII, 321.

djoumeri, m., Montbéliard (Doubs), Contejean, Gloss.

jou-mairi, m., boue-solit, beû-solaî, franc-comtois, DArTo1s, Etudes des pat., 1850.

orilyettes, f. pl. (= pendants d'oreille: les fruits), wallon, FELLER.

"u'a spina, uva crespina, uva marina, italien, PIstaneld, 1577.

crespino, berberide, bérbero, italien.

berberi, m. sing., sicilien, Cupanı; Mortillaro. - piémontais, Capello. barberi spinnsu, sicilien, Scuderi, Boschi dell' Etna, 1825.

crespin, nord de l'Italie. - Belluno.

craspein, Plaisance, Bracciforti.

crispi, Brescia, Zensi.

gherspéin, Bulogne, Coronedi-Berti. - Reggio, Vocab. regg.

yraspin, Belluno, Nomi adoperati.

scarpin, Trévise, Belluno, Nomi adoperati.

spinella, Corse, Requies, Cat. des reyjétaux de la Corse, 1852.

spima acida, sicilien, LAgrsi. - sud de l'Ital., Nomi adop.

spina, spina rimosa, Sud de l'Italie, Nomi adop.

spinau, piémontais, CAPELlo.

spin vinet, Turin, Nomi alloperati.

spir acit, zinevrin, Nilan et Côme, Nomi adoper.

spin d'asen, Sondrio, Nomi adoper.

spin dalla croce, Trévise, SACCARdo.

spinne d' la lerr, Asti, Canisola. [J. C.].

spina morta, spiner yraspin, spin de graspin, Belluno, Nomi adoper.

spin ruerz, spin di cros, Frioul, Pirona.

u'a de spi, crespi, Brescia, Nomi adop.

(1) Ce nom usité à Saint-Véran équivaut à éveilleur, c'est-à-dire qui éveille par son acidité. Chabraxd. 
ua de la madona, Brescia, Melchiori.

eua de spi, euéta, Bergame et Crémone, Nomi adop.

ua d' la levr, environs de Turin, Re.

nva d' la lever, pan di giari, Cuneo (Piémont), Nomi alop.

uveta rossa, Vicence, Nomi adoper.

ua spinela, salgher, pan e vin, Belluno, Nomi al.

uvetta, Aquila, Nomi adoperati. - Terra di Lavoro, Petagna.

ua graspina, Padoue, Patriarchi.

scotanella, Ancône et Macerata, Nomi adoper.

cèdri, Frioul, Pirona.

corignanello, Terra di Lavoro, Petagna, 1827.

espina vinetta, catalan, Lacavalleria, Guzophylacium, 1696.

coralets, catalan, Costa, Flora, 1864.

berbero, agracejo, alarguez (1), espagnol.

berberis, bérbiles, esp., J. Victor, Tesoro, 1609.

arlo, espo, Fragoso, Cirurgia universal, 1621 ; 0udin, Trésor, 1660.

ubas de arlo (les fruits), esp., Alosso, Privilegios $p$. mugeres preñadas,

1606.

alro, Sierra Nevada et Sierra Tejeda, BoIssien, V'oy. bot. d. le midi de l'Espagne, 1839.

agruzejo, alynese, Sierra de la Nieve (midi de l'Espagne), Borssier, Voy.

bot.

bérbero, aragonais, BonAo.

Iracila, macrish de riuri, roumain, Brandza, Limba botanica, 1882.

dracinu, macrish $\left.{ }^{2}\right)$ de spin, roumain, Ciнac, Dict. daco-rom.

acrish roshu, roumain de Transylvanie, Fusz. - roumain, HASDEL.

macrish-spinos, HASDEU.

bersich, persich, versich, versick, paisselbeer, weindling, weinling,

wildweinieb, latzin ore, lirispele, erbesip, erbesipp, erbsal, kolshals,

blutwur $\approx$, anc. et moyen haut all., Diefenbach, Gloss. med. lat.

crispil, anc. haut all., Germania, 1888, p. 302.

saurach, weinlägelin, weinleyelin, erlbsel, anc. et moyen haut all.,

Diefenbach, Gloss.; J. Agricola, Medic. herb. libri duo, 1539.

surich, anc. haut all., PIPER, Altd. Pflanzenn.; Cordus, Botanologicon, 1553 ; Germania, 1881 , p. 402.

sauerdorn, anc. haut all., EBER et PEUcER, Appellationes, 1556.

(1) De l'arahe al-arghîs.

(2) Macrish signifie oseille. 
erbesop, erbesich, erbesus, berbis, prümel, peyssel, moyen haut all., Pritzel et Jessen, Volksn.

versichdorn, beerlorn, salsendorn, essigdorn, prummelbeeren, erbshofen, weinnügelein, weinzäpfel, wienschierling, wütscherling, allemand, Nerich, Lexicon, 1793.

erbsele, dornholz, allemand, GRIMм.

saurauch, reiselbeeren, heinselbeeren, allemand, DuEz, 1664.

sauerdorn, berbersbeeren, berberitze, barberitze, allemand.

weinschülling, allemanı, Rosexthal, Syn. pl. diaph.

beltlerkraut, rifspitzlueere, spitzbeere, erbselnwurs, canton des Grisons,

Pritzel et Jess., Volksn.

spinatsch, Engadine et Grisons, Durheim, Schweiz. Iliot.

gälhagel, canton d'Ippenzell, Durheis, Schwei:. Idiot.

reifleere, Suisse allemande, Durhein, Schw. Idiot.

erbsele, erbsele-beere, spitzbeere, reifspitzbere, gelbhagel, Suisse alle-

mande, Stalder.

schwillern, pl., Valais, Stalder.

frauasurampfara, buabalaub, gailhageldorn, erbsalabeer (les fruits), gitzilveer (les fruits), canton de Saint-Gall, Wartuan.

sperberbeer, Henneberg, Pritzel, Volksn.

gischyalizen, bavarois, Schielder, Bayer. Wört.

rebarber, Prusse, Frischibre, Preuss. Wört.

weinscherling, weinscheidling, Autriche, Pritzel et Jess.

zi:erl, weinscharl, essigscharl, ersidl, essigplütwerl, Basse-Autriche, F. HöFER.

baslbeer, masselbeer, Carinthie, Zwanziger, Verzeichn., 1888.

rüht aegresch, schiesbriger aegresch, allem. de Transylvanie, Fusz, Trivialnamen.

Ireidar, Luxembourg, J. WEBER.

essigtanyen, Grosbliderstroff (Lorraine), rec. personn.

suerboom, anc. flam., DodoxaEus, 1644. [A. DE C.].

zuurloorn, hollandais, VAN DALE. [A. DE C.],

berberisse, flamand, DE GORTER.

sause boom, anc. flamand, Diefenbach, Gloss. med. lat.; Dodonaeus, 1644.

berberine hout, flamand, Roucel, Flore du Nord; POEderLÉ, Manuel de l'arboriste, Bruxelles, 1788.

barbarine, barbarinekens, flamand, DE Bo, Westrl. Idiot.

bulberies, berberies (les fruits), anc. angl., Mowat, Alphita.

barberry tree, angl., Cotgrave, French dict., 1650.

barberry, anglais. 
berber, Ecosse, Jamiesox, Diction.

pipperidge, Est de l'Angleterre, Fonву, Vocab. of East Anglia, 1830.

guild, guild tree, Selkirkshire (Écosse), Jamiesos, Dict.

jaundise tree, jaundice berry, anglais dialectal, Britter, Dict. of plant-

names.

jaunders tree, West Somerset, Elworthy, W. Som. Word-book.

pren melyn (= arbre jaune , drain yspinys (= épine épineuse), gallois,

J. DAviES, 1632. [H. G.].

greol ysbin, yspinwydd = arbre à épines), gallois, Merldygon .Myddfai.[H.G.]. draenen ysbinus = arbre épineux), gallois, Hugh Davies. [H. G.]

pren y clefyd melyn (= herbe de la jaunisse, gallois, Walters. [H. G.].

barbray, preas nan gear dhearc (= sour berry-bush), preas deilyneach

= prickly bush), gaélique écoss., CaMeron. [H. G. ?.

barbrog, irlandais, Camerox. [H. G.]

sperneenn-trechonnek $=$ épine d'oseille), breton, L'ARMERIE. [E. E.].

surtorn, berberis, danois, Jex.sex-Tesch, Nord. pl.

babotrsounia, babtorina, breberika, cesmiga, česmile, česmine, čsliga,

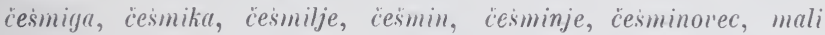
cepcesac, mali c̈eviezac, trpkovina, żutelinka, zutiliorina, ¿́utika, zutokora, turslia loza, dulji simsir, simsirilia, kiseli trn, krespin, mersin, morluan, pesji parkel, iraṡji parkelc, serbocruate, šlek, Juy. im. bil.

drä̈, piunik, telièque, A. MëLler, IV. symonym. Namen, 1866.

dristal, tchèque, šluek, Jug. im. bil.

wanżary, tchèque, LiNue, Sl. jez. polsh.

oskorusha, slavonien, Linde, St. jez. polsk.

certory stomik (= Teufelstamm), wende, Vox Schllexburg.

bahrbeles, Courlande, Llmaxi, Lett. Wört.

borlus, berber'ys, kalina wloska, polonais, LINDE.

molirych, barbaris, petit russien, c. par II. Th. Volkor.

kisliza, russe, Falk, Top. Kenntn. d. Russl.

paklun, russe du Don, Falk, Top. Kenntn.

barbaris, kisloi tern, russe, PIstohlions, Namenverzeichn. I. Holzarten, 1797.

srishk, dzor, ked:ochour, parrpourr, arménien, ALshax. [ER. L.].

saparlar, aranzibi, erkhalatch, basque. [J. V.].

sóskafü, magyar, Fusz, Trivialnamen.

parberitsil, esthonien, Pistoнlков, Namenverz. d. Holzarten, 1797.

barbarîs, amîrbaris, arabe.

zereschk, persan, Pharmacopæa persica, 1681, p. 248.

zirichl, Ghilan (Perse), Снорzк0, Le Ghilan, p. 64. 
zarschak, persan, DRAGENDoRFF., p. 48.

mouberidj, persan, MENinski.

anbérbaris, turc. - persan. - arabe.

qaden touslouyhou, turc, Mallouf, Dict.

qalentoüz, hardün bahar, turc, LEcLERc, Révélations, 1874.

kozachur, géorgien, Falk, Top. Kenntn. d. Russl.

sirch, sirch modun, Boukharie, Falk, Top. Kenntn. d. Russl.

sirk, siritch, Turkestan, DragendoRfF, p. 48.

sare ayatsch (= lignum citrinum), tatare, DRAGENDonfF, p. 40.

chatyn tusluk, tatare criméen, Steven.

chadyn tustuk, tatare criméen, Pallas, Observat. en Russie, 1793, II, 394. cidul fibil (= raisin d'ours), avare (Daghestan), SCHIEFNER, Ueber das awarische, $186^{\circ} \mathrm{2}$.

hashmal, chochar, zirishk, Pendjab, Gamble, Manual of indian timbers, 1881 .

\section{2. - VOMS DE LA RACINE.}

L'écorce de la racine de cette plante était autrefois en usage pour la teinture et la pharmacie, on l'appelle :

alarguez, ancien espagnol (1), Dozx.

laryjs, portugais, Dozx.

alaryhis, aryhis, arabe, d'origine berbère, DozY.

athrîr, arabe, Freitag.

$a b z a r$, arabe, IBN BEITHAR.

arghis, berbère.

\section{3. - L'ÉPINE-VINETTE ET LA ROUILLE DES BLÉs.}

Les blés qui se trouvent dans le voisinage de plantations d'épines-vinettes sont ordinairement atteints par une sorte de rouille qui compromet la récolte. Aussi les paysans ont-ils en aversion cet arbuste et le détruisent chaque fois qu'ils le peuvent, mème sur le terrain d'autrui. - Voyez à ce sujet :

Manqus, Observat. sur l'influence nuisible qu'on attribue à l'EpineI'mette sur les moissons (dans : Trav, acad. de Rouen, 1818, p. 90-98).

Fullet. de la Soc. botan. de France, t. XVI 1869 , p. 331 et suiv.

Revue de botanique, t. IV (1885), p. 181 et suiv:

Cuziat, De l'influence de l'Epine-Vinetle sur les céréales dans : Soc. l'émul. des Côtes-du-Nord, 1883, p. 2i-32).

(1) De larabe al-ârghiz. Voyez Dozs, sub verbo alarguez. 


\section{1. - ISAGES.}

Le fruit de l'épine-vinette est une baic de couleur rouge; la pulpe est gorgíe d'un suc acidulí, astringent, de coulenr rosée. Ce fruit se présente en grappes composées d'une douzaine de baies dont la forme oblongue et la couleur rouge offrent l'aspect le plus gracieux; on le mange rarement cru. le plus souvent on en prépare pour les usages économiques des conserves et des confitures très délicates et très saines; ces confitures sont l'objet d'un commerce assez considérable ì Chanceaux, près Dijon.

Les baies d'épine-vinette, lorsqu'elles sont encore vertes, sont employées a l'instar du citron, ef notamment dans le Nord, pour relever la saveur fade de certains mets; confites au vinaiggre, clles remplacent les câpres comme assaisonnement; leur extrême astringence les a fait ranger parmi les substances qui entrent dans la composition de la préparation officinale qui jouit de cette propriété an plus haut degré, Diascordium.

Colverchel, Traité des fruits, 1839. p. 308.

$$
\text { 5. - FOLKLORE. }
$$

C'est avec l'épine-vinette qu'on a couronné Jésus.

Pays wallon, Feller, p. 206.

$$
\text { IBERRERIS AETAESSTS. (PRESLI. }
$$

berberi spinusu, Catane, Nomi adop.

spina cristi, spina santa, Sardaigne, Moris, Flor'a.

dmamai, arabe algérien, Muxbr, Noms ar. des $p l$.

BERRERIS HISTANICA. (BOISSIER).

thasyouart, kabyle, HAxotEAu, La Kabylie.

\section{BERREIISS CRETICA. (LINNE).}

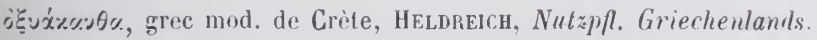

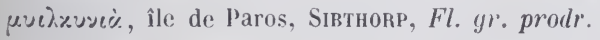

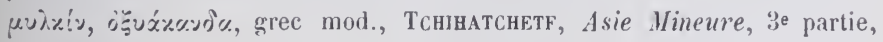
1868.

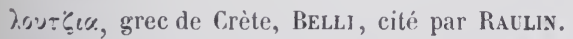

jasmin de Crète, français, Saint-Germain, Manuel des Végétaux, 1784.

spina cristi, spina santa, Sardaigne, MoRIs, Flora. 


\section{BERBERIS LYCIUII. (RoyLe).}

1. - Cette plante croît dans l'Inde. Les anciens en tiraient un médicament, appelé lycium ( $\lambda \dot{u} \varkappa(0)$ ), principalement usité dans les maladies des yeux.

En arabe, selon Dozy, on appelle cohl khaulân un suc tiré d'une espèce de Berberis. (Ce suc semble correspondre au lycium des anciens).

Voir pour l'historique de cette substance :

Flückiger and Hanbury, Phamaacographia, 1874, p. 34-35.

J. Y. Simpson, Notes on some ancient greek medical rases for containiny Lycium. Dans le T. I. des Proc. of antiqu. of Scotland, 1852, réimprimé à part en 1856 et dans le T. II (1872 des Archrologic. Essays de l'auteur).

RoYle, On the Lycium of Dioscorides (Lim. Trans. XVII, 1837, 83 .

Journal de pharmacie, $\mathrm{V}, 88$ et 9 ?.

BaLl, Identification of the plants of India knou'n to early greek authors (dans Proc. of. the roy. irish Acad. 1885 . p. 340.

2. - "Licium; aucuns dient que c'est une gomme, mais ce n'est pas, car c'est le jus d'une herbe appellee ainsi licium... L'en l'appelle autrement oculus lucidus ou oeil luisant pour ce qu'il rent les reulx clers et reluisans. »

$\mathrm{AV}^{*}$ siècle. - Camus, L'op. salernit., p. 84 .

\section{EPIIEDIU.I ALPINL.I. (LINNÉ).}

chapeau d'évêque, m., épimède des Alpes, m., français (1). lipicu, biskupsca kapa, vimčel, serbo-croate, šulek. afighidioûn, arabe ș̣rien, BergGREN, Guide arabe, 1814.

\section{LEONTICE CHRISOGONUII. (LINNÉ).}

\%ouróyovoy, grec anc., Dioscoride.

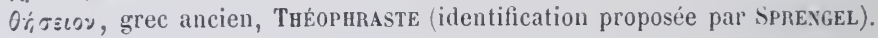

\section{LEONTICE LEONTOPETALUM. (LINNÉ).}

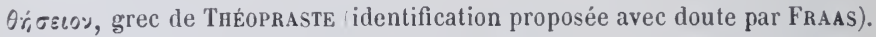

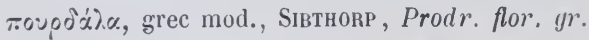
$\tau \zeta \dot{x}$ кpes, grec mod. de Chypre, IDEM.

(1) La plante n'existe en France qu'à l'état cultivé . 
leontopetalon, leontopetalum, anc. nomencl., BaUhin, Pinax, 1671.

pedi di liuni, sicilien, LAGusi, Erbuario, 1742.

arudzatjank (= griffe de lion), arménien, Alishav. [ER. L.].

thayteya, arabe syrien, BergGren, Guide.

\section{NYMPHÉAGÉES.}

\section{NYMPHAEA ALBA. (LINNÉ), NUPHAR LUTEU.1., (S.ITH). -} LE NÉNUFAR (1).

\section{1. - CEs DeUX espèces SONT habituellement CONFONDUES SOUS LES NOMS SUIVANTS :}

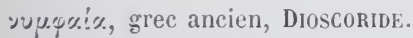

protea, caccabon, lotometra, rhopalon, androgynon, hydragogon, hera-

cleon, andreas nerion, grec, IpulEILs (auteur latin du Vo ou VI s.,)

De Medicaminibus.

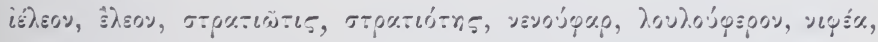

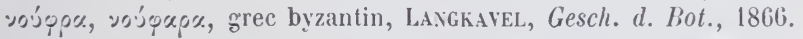

vo'spapos, grec mod., Somalera, Tesoro d. lingua greca volg., 1709.

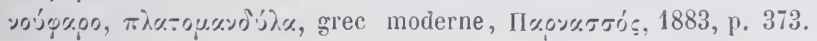

nymphaea, latin, P'LINE.

clava Her'culıs, latin du IVe siècle après J.-C., Marcellus empiricus.

mater herculania, alga palustris, papacer palustre, clavus Veneris, aqux

ducta, latin du Ve ou Vo siècle apr. J.-C., Aplleius, De Hedicaminibus. Edit. de 1788, p. 232 .

ungula caballina, anc. nomencl., Diefesbach, Gl. med. lat. s. vo Mufra.

- Lat. du IXe s., ED. Boxwet.

ungula caballina aquatica, anc. nomencl., MowAT, Alphita; DE Bosco,

Luminare majus, 1496, fo 39, verso ; Cordus, Botanologicon, 1533.

numphea, lat. du IX'e s., ED. BoNNET.

nenufar, farfara, anc. nomencl., DE Bosco, Luminare majus, 1496, fo 39, verso.

(1) C'est à tort qu'on écrit nénuphar, le mot venant de l'arabe ninoûfar. Le ph de nénuphar est sans doute dû à l'influence du mot nymphaea. 
caulis aquae, alya palustris, yranum sponsi (le fruit), anc. nomenel., DE.

Bosco, Lum. majus, 1496, fo 33, recto.

neniphar, cacabus veneris, lilium aquaticum, nimphea, limphea aquatica,

luppacium aquaticum, luppacium mujus, papaver palustrum (1), alyu

palustris, nomenclat. du Mọen-Age, Mowat, Alphita.

nemupharinum, anc. nomencl., A. Lusirants, Enarrationes, Venetiis, 1533 , p. 228. [J, C.]

heruclea, diyitus Veneris, ralir Herculis, ane. nomencl., Gefraroy, Mat. méd., $17 \div 0$.

nénufar, m., némuphar, m., français.

neneuphare, anc. franç., Vallanbert, Mnnieve de nourrir les enfans, 1565 ,

p. 265.

neufur, anç. franc., Ronsard cité par Littrì.

nenufa, m., Aube, Des Exangs, Noms pop. lles plantes.

nuphar, m., Bessin, Jonkt, Flore populaire.

mufa, m., mufii, m., Aube, Des Etaxgs, Noms pop. des pl. - Haute-

Marne, comm. par M. A. DAGun,

hunifa, Oise, Graves, Catalogue des pl.

unéfa, Vexin, Joret, lilore.

unifa, Bessin, Joret, Fl.

lusiper, Saintonge, Jonain, Dict. du pat.

nymphée, f., anc. franç., Cotgrave, French tict. 1650 ; - Evon. Philiatre,

Tresor des remedes, 155i, p. 100.

nymphe, f., anc. franc., BÉrodLde DE Verville, le moyen de parvenir

(ver's 1610), édit. Jacob., p. 323. - Centre, Jaubert, Gloss. du centre ninfea, Nice, Risso, Hist. nat., 1826.

ninfiar, Semur en Auxois (Còtes-d'Or), c. par M. H. Marlot.

nimfeo, f., Nimes, Vincens, Topographie, 1802.

ninfèio, f., provenç. mod., Mistral, Tres. - Saint-Rémy (B.-du-Rh.),

II. Girard, lis Aupiho, 1878, p. 53.

erbo d'infer, provenç. mod., Mistral, Tres. d. fel. - Nord du départ.

des Basses-Alpes, e. par M. E. Platchid.

erba dou diable, f., Bas Dauphiné, Moutier.

nimfo, f., ninfo, f., provenç. mod., Garidel. - Bouches-dn-Rhône, Ville-

xecre. - Environs d'Avignon, Palun, Catal. - Var, Anic, Consid, sur

Brignolles; Hanry, Catal.

lys d'euu, m., lys des étomgs, m., français ancien et mod.

(1) Le manuscrit cité par Mowat donne papaver plaustrum. C'est une erreur de copie de mêne que galla palustris pour alga palustris. 
lys d'étany, m., français, Traiclé de la siguature, traduil de Crollius,

Lyon, 1624, p. 50; Nme Fouguet, Suite du recueil des remedes, t. II, 1701 , p. 360.

liri d'aygua, f., Luchon (Pyrénées), sAcaze.

liri d'aigo, m., languedocien, Duboul.

rose d'estan, f., anc. fr., A. CoLiv, Hist. des drogues, Lyon, 1619, 1. 127. rose d'eau, f., anc. franç., Duchesne, De stirpibus, 1544; Вконол, De stirpibus, 1541.

rose des mares, f. Manche, Besroc, 1860.

irose d'étang, rose les étangs, f., Vosges, Halluant, fil.

rose des lacs, f., Doubs, Bealquier, Vocabul.

roj-punnie = rose punaise, rose puante), Nièvre, c. par M. AcH. Mullex. girofflee d'eun. f., anc. firanc., Diez, 1666.

choul.r de eane, chou d'eau, m., anc. franc., Dechtsse, De stirp., 1511. chou de rivière, m., Eurt-et-Loir, rec. personn.

cuoulusso, f.. (= chou, avec un sens péjoratif, Gers, comm. par II. II.

DAignestoIs.

tulipe d'étanq, f., Vosges, Hallant, Flore.

parot de riviere, III., env. de Domfront Orne), c. par II. Mvi. Cheribier. courge d'eau, f., Côte-d'Or.

coulcharusso, f., Caussade Tarn-et-Garome, LAconbe, Lambruscos, 1879, p. 201.

coutsurasso 1., Bas Quercy, comm. par M. J. D. Yrind.

coururasso, f., langucdocien, Por MARĖDE, IHunel des termes, 1860.

coujarasso, f., languedocien, Dıвоц.

cujurusso, f., Dunes, arrond. de Moissac (Tarn-et-Gar., ree. pers.

coujous d’ä̆go, m. pl., (= petites courges d'eau) Castres, Colziné, Dict.

pat., 1847. - Tuulouse, Torrsos, Flore. - languedocien, Dtboul.

coloquinte, f., Haule-llarne, comm. par M. А. DAguis.

qourde, f., fourdon, m., Aube, Des Etangs.

cruche d'eau, f., crujlieon, In., Saintonge, Josalx, Jicl.

crugeon, m., Anjou, Desvatx, Flore. - Chef-Boutonue Deux-serres),

Beauchet-Filleat, Ess. sur le pat. poit.

cruchon, m., Anjou, Desvaux, Flore.

pompe, t., Iube, DEs ETANGs.

aplomple, f., appolompe, f., applombe, f., wallon du $\mathrm{Xl}$ siècle, J. Canus,

Un manuscrit namurois.

bouteille, , f. (Ie fruit), Bresse chìlonnaise, Gunlemis, Glossaire, 1869. -

Haute-Marne, comm. par M. L. IUbniot. - Charente-Infér., comm.

par M. E. Lemarié. - Nièvre, comm. par M. M(IILle Millien.

boteyj d'éw, f., Malmédy Pậs wallon), Feller. 
cabucéou, m., cabrecelo, f. , provenç. moderne, RÉGuis.

baratte, f., Normandie. - Maine. - Anjou. - Berry. - Haute-Bretagne. bat-beurre, (1' (le fruit) Haute-Marne, comm. par M. L. AUBrior. - Aubignysur-Nère (Cher), rec. pers.

beurrière, f., Yonne, Jossien, Dict. pat. de l'Yonne.

burelte, f., Aube, Des Etaxgs, Noms luly. - Haute-IIarne, comm. par

I. A. Dagur:

bourette, f, pays d'Auge (Normandie), JoRET, Flore.

barette. f., barotte, f., Normandie, JoRet, Flore.

batiaur, baratiaux, Domfront (Orne), c. par M. Atg. Chevilier.

battoirs, pl., salière, f., Soulaucourt (Haute-Ilarne), comm. par II. l'abbé MARCHAL.

plateau, m., Aube, Des Etaxgs, Noms vulg. - Haute-Marne, comm. par

II. A. Daglix. - Loiret, comm. par M. L. Malox. - Allier, Olivier,

Flore pop. - Luxembourg wallon, Dassor, Dict. wall. - Centre, Boreau, Flore.

platiau, m., Bar-le-Duc (Meuse), rec. pers. - Romorantin Loir-et-Cher, rec. pers. - Nièrre, c. par M. A. MiLliex.

platê, m., wallon, FELLER.

plaquiau, m., Yonne, Jossier, Dict. - Loiret, comm. par II. L. Malox. platuche, f., Libourne (Gironde), c. par II. L. DERAND-DÉGR.1NGE. - La-

bouheyre (Landes), c. par II. F. ARNALDix.

plat-plat, m., Aube, Des Etangs, Noms v'ulg. - Haute-Yarne, comm. par

II. A. Daguin.

plapat, m., paplard, m., Aube, Des Etaxgs, Noms vuly.

parapluie, Inneville-sur-Seine (Normandie), JoREt, Flore.

chèpé d' bu, m., (= chapeau de bouf; les enfants, par amusette, font arec

la large feuille du nénufar, un chapeau aux bœufs), Plainfaing (Vosges),

rec. persomnell.

nappe, f., Ineuil (Cher), rec. pers. - Berry, JALBERT, Gloss. du centre. creipo d'aigo, f. limousin, Mistral, Tres.

volet, m., Maine. - Anjou. - Bourgogne. - Poitou. - Vendée. -

Haute-Bretagne.

volette, f., Haute-Bretagne, comm. par M. SÉBILl0T. - Charente-Inf., comm. par I. E. LE.LARIÉ.

trocheux de ru, m. (= tranchoir de ruisseau), Romont (Vosges), HaILlaxt, Flore.

(1) Ce mot signife baratte. 
tranchoir, m., Bresse chàlonnaise, Gurllexis, Glossaire, 1862.

tranchou, m., Charente-Inf., comm. par M. E. LEMARIÉ.

langue de bœuf, f., Haute-Bretagne, comm. par M. P. SĖBillot.

ongle caballine, f., anc. fr., Evon. Philiatre, Tresor des remedes, 1555 ,

p. 100 .

pourine, f., pourique, f., Marensin Landes), comm. par M. l'abbé

V. Foix.

pas de bœuf, m., pied de bœu, m., Saintonge, Jòxas, Dict. saint.

pè di chil'aou, m., Ouest du Lot-et-Garonne. c. par M. l'abbé L. DARDY.

pied de gva, m., Bessin, JoRET, Flore.

pè de mul, m., languedocien, Duroct.

pé de mulo, m., Toulouse, Tounsos, Flore.

ped de muelo, provenc. mod., Mistral. Tres.

pas d'àne, m., Chàtenay Seine-et-Marne, rec. pers.

pas d'asne de murais, m., pus de cheval des marais, m., anc. franç.,

DeEz, Diz. ital. franz., 1678.

patte d'oie, f., Cotentin, JoRET, Flore.

pied $d$ piret, m. pied d'oie), canton de Périers (Manche, rec. pers.

poupas, m., Anuay-sur-Serein (Yonne), Jossier, Dict.

tourteaux, m. pl., Charente-Inf., comm. par M. E. LevariÉ.

pareil, Loire-Infér., Ed. Richer, Descr. pittor. de la Loire-Inf., p. 50.

läches, f. pl., lôches, f. pl., Haute-IIarne, comm. par II. A. DAGuis.

trimuau la racine), anc. franç., J. CAycs, L'opera salern., $\mathrm{n}^{\circ} 328$.

tocquons, gomé, morêhe, Vosges, H.allaxt, $\mathrm{Fl}$.

montre, miai, solire. Vosges, Hallant, Flore.

dame d'onze heures, f., Bessin, Jonet, Flore.

vié malaou, viet malaou (=v. malade ${ }^{1}$, envir. d'Ivignon, PALlx. -

Arles, Latgier de Chartrouse.

rabarljöi $=$ rabat-joie, m., récène du rabadjoï, wallon, Feller.

herbe de cur'és, f., Semur en Auxois Côte-d'Or, c. par I. H. M.ırot.

herbe aux moines, f., Haute-Marne, comm. par M. A. DAGus.

racanette, f.. racanolte, f., Aube, Des Etaxgs, Noms vulg.

tabatiere le fruit, Pas-de-Calais, Lecesse.

reine des rivières, f., Aubigny-sur-Nère (Cher), rec. pers.

ninfea, italien, Ambrosini, Phytologia, 1666. - Env. de Turin, Re, 1825. nenufaro, nenufar, ital. du XVIe s., Cayus, Studin di lessicogr., p. 10.

(1) On a dû dire à l'origine : erbo de viet malaou. - L'explication de ce mot se troure dans Garidel, Hist. des $p l$. d'Aix, 1716 : " on se sẹt des racines en décoction pour adoucir et tempérer l'ardeur des urines dans la gonorrhée. 
nemufero, nunnufero, nanufero, nemunfero, carfuno, cuppero di padule,

italien, Targlon, Di:. botanico.

capellazzi, Vérone, Pollini, Flora ver., 1824.

capllazz, Ferrare, Ninxini, Vocab. ferrarese, 1705.

lajero, tujerasso, ital., Le.r icon italico latimum, Taurini, 1618.

tagrero de vale, Padoue, Pitnisacin.

tajée, m., capellin, milanais, Chenubix.

tuylieruz:o, Bologne, Ambrosini, 1666 .

tajer d'ucqun, cupplizs, Plaisance, Br.scifort, Floru piucent.

tuilon, Tessin, Poldini, Flor a veronensis.

nimfu, Brescia, Mecchori, Vocub. bresc.

laverlon, milanais, BANFI, Vocab. milan.

cosarute, Frioul, Pirona, Vocab. firiul.

crocoriga de acqua, Sardaigne du Sud, Monss, Flor'l surd.

liri sarvaj, piémontais, Colls.

lillu de acqua, Sardaigne, SPAno.

slaluis, nacisse, anisse, Brescia, Zensis.

ciupin d'mula, env. de Turin, Re, 1825.

folla do ar, lirio de agua, lirio acuitico, espagnol.

hiyo del rio, escudéte, nenufar, yolfiino, esp., J. Victon, Tesoro, 1609.

coberteru de ayuu, esp., Alosso, Privilegios para inugeres preñulas, 1606. coverterll, Tolede, IDEM, idem.

aduryua del rio, yolfan del rio, figo del rio, escudetes del rio, espagnol,

Lusitanus, Dioscorilis medica muteria, 155.

erbu do ar, tapadeiras, galicien, Cu veIro, Dicc. yall.

erba d'o aire, galicien, Valladares.

lliri de ayguu, catalan, LACAvalderia, Gillopleylacium.

nimfu, catalan, Costa, Floru de Cutul.

erbo den Jofa den est l'abréviation de de $+e n$; îles Baléares. Figukra. yolfüo, portugais, Barbosi, Dict. lusit., llil1. gelpsano, portugais, Duchesse, De stirpibus, 10\%4.

uufur, nenufar, roumain, Ciнsc, Dict. daco-rom.

plutu, roumain de la Valachie, comm. par II. A. Gorover.

plumiera, roumain de Transylvanie, comm. par II. A. Gokover.

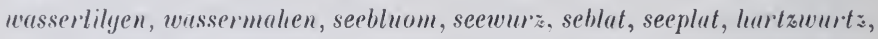

hurstrung, leerculiswurtz, liallerwurtz, yrensinck, anc. all., DIEFE.ВасН,

Gloss. med. lat.

seelblumen, anc. all., Grins, Wört. sub verbo Ilaarwurs.

bienenmörder, hörstrany, hörwurs, leulenwurz, liollerwur , lıckermögen, seecrenich, wassermun, wassermolinblume, wasserwur zeblat, anc ill., Pritzel et Jessen, Iollisn. 
seblad, all. du nord-ouest, X11 ${ }^{\circ}$ s., GALLÉE.

grensich, anc. h. all., Germania, 1888, 307; Zeilsch. f. deutsch. Alterth.

1853, p. 397.

colerwurtze, anc. h. all., Germunia, 1888, p. 307.

seeblume, seerose, wasserrose, allemand.

wasserveil, wasserveilge. wasserviol, allemanl, DuEz, 1664.

kolbwurz, allemand, Grim.

wassertulpe, wassermolhen, wassermünnchen, wussermohn, wasserlilie, seepuppen, poppelke, tolllilie, liehlwur: kohlwur's, mürmellien, kannerplumpen, allem., Nennich, Polyglotten-Lericon.

sehekenlien, Saxe, Conds, Botanol., 1533.

kahnetocken, Saxe, Pritzel et Jess., Volksnamen.

essiglirügle, schwindelwur\%, Augsbourg, P'Rtтzel et Jess., Volksn.

plumpen, tollilgen, nixblurien, seekandel, Silésic, Рват\%. el Jess.,

Volksn.

wassermänngen, Saxe et Thuringe. Pritz. et Jess.

tollingen, Thuringe, P'rIT\%, ot Jess.

Irumm'lstöck'r, pl. (wegen der Aehnlichkeit des Stieles mit den Trom-

melstöckern, Altmark, DinseIL.

waol'rtulp'n la fleur), Altmark, DANNeIL.

watarstedi, anc. all. dialec., Tijilschr. 1'. nederl. Letterl., Leiden, 1894.

wissertulepant, f., Luxembourg, J. Weber.

puppen, Mark bei Sommerfeld, Pritz. et Jess.

poppelhlome, poppel, pöppellien, environs de Munster, Focke, I'flanzennamen, 1870.

liemke, kenke, bubbellie, Frise orientale, Focke, Pflanzenn.

lodlienbläler', nord-ouest de l'Allemagne, Focke, I'flan:.

mummel, kanne, kmmlie, Prusse, Frischbier, Preuss. Wint.

kennken, kohntjen, Duché d’Oldenbourg, STrACKERJAs, Abergluube aus Oldenbury.

pöppeln, paupslien, environs de Munster, Strackerjan, Idem.

maariose, Eifel, Wintgen, Vegelation der Eifel.

fröscheblumen, Oberland hernois, Pritz, et JEss.

lirampfworzel, mossroasa, wasserrosa, canton de Saint-Gall, Wartyans. lätschblätler, Mlsace, MAPP's, IIist. plant. alsatic., 1742.

puppenblader, buotterkärnen les fruits), Westphalie, Laxdors, Westph. Pflanzenn.

botervat, flamand, Van Heurck, Flore médicale belge. [A. DE C.]. dokke, amberblaren, ampelblaren, plompe, waterplompe, waterroos, wompelbluren, kaasbloem, llamand occidental, DE Bo, Westvlaamsch Idiot. d. de C.]. 
kallemoei, fleschkens, trommelstokken, fl, Pays d'Alost, c. par M. А.DE Соск. plompen, waterrozen, waterleliën, waterkruitien, meerbladeren, hollandais,

Oudemans, Flora et Van Dale, Wdb. [A. DE Cock].

pompe bloemen, Groningue, DE GoRTER.

ea docca, anglo-saxon, EarLe, Engl. plant-names.

ea docce, docce, anglo-saxon, Cockarne, Leechdoms, etc., 1866.

waterloklie, edoklie, anc. angl., Mowat, Alphita.

colloncroh, anglo-saxon, Cockarne, Leechdoms etc.; DiefexBaci, Gloss, m. lat. chur'n, butterchurn, brandie-bottle, lily-can, water coll's foot, flatter-

dock, candock, cumbie-leaf, water lily, bobbins, dans les difrérents dialectes de l'Angleterre, Britten et Holland, Plant-names.

baditis, gaulois, Marcellus EMpiricus (fin du IVe siècle après J.-C.). [H. G.] duilleog-baite, irlandais. J. КЕоGн, 1735. [H. G.].

bior ros (= water-rose), cuirinin (= petite cruche), gaélique écossais,

C.uneron. [H. G.].

breilen y dwr (= rose d'eau), rhos y dwe (= rose d'eau), gallois, Meddigon

Myddfai. [H. G.].

loa-dour (= cuiller d'eau), breton, MaréchaL.

loa-zour, breton, LE Gonidec; Troude; Finistère. - Crouan. [E. E.].

sjöbloma, sjörosur, kärnrosor, vattentulpa, bäkleblomma, nälikdälilior,

nälikros, nilikeblomm, sliöllblad, pompai, blokker, suédois dial.,

JENSSEN-TusCH.

vasrosa, akaleje, hestehov, lialblom, norvég., Jexssex-Tusch.

kjürlingelirus, åkande, äblomme, bxkblomme, koklokike, danois dial.,

JENSSEN-TUSCH.

lelinju, lekno, tchèque, PALKowitśch.

grzybien, gr:ybienie, wodna lilia, polonais, LINDE.

gaska, lierzentia (1), polonais de Prusse, Treichel, Poln. Vulgärnamen. kubuszczack, russe, MENTzel, Lexicon, 1715.

kouvchinka, liouvchintchiki (= petits pots), vodianaïa lilia (= lys d'eau),

russe, Schialhausen. [Th. V.].

latattia, petit russien, c. par M. Тн. VoLkov.

nonopar, nounoufar, th:ékan chot (= herbe de poisson), kokorr draghik,

harsnamaten (= doigt de l'épousée), arménien, Alishax. [LR. L.].

ighebederra, basque, Fabre, Guide franc. basque.

sosach, tatare, Pallas, Voy. en Russie, II, 502.

niloufar, ninoüfur, noûfar, a rabe et persan.

nimofar, Turkestan, DRAGENDoRFF.

(1) Kerะenka = Butterfass, weil Frucht ihm gleich. 
âboû, persan, Meninski

ulufer, turc, BARBIER DE MAYNARD, Dict. lurc.

L'arabe niloufar (d'où vient notre mot nénufar) est dérivé lui-mème du sanscrit Nîlôtpalam qui sert à désigner une espèce de nymphea célèbre dans l'Inde, le lotus bleu (de nila $=$ bleu et de utpalam = lotus, nymphea). Niloótpala est devenu dans les dialectes dérivés du sanscrit nîluppal (CHILders, Dict. of the pali language, 1875, donne comme nom pâli du lotus bleu nîluppalam). De nîluppal les arabes ont fait nîlufar. (L'arabe n'ayant pas de $p$, le change régulièrement en $f$.

Le nom du lotus bleu a donc été donné par extension, à d'autres nyınphéacées.

MARCEL Devic, dans son Dict. étym. avait bien reconnu dans le mot nîloufar, le premier élément, c.-d̀-d. nila (bleu) mais il s'est trompé sur le second; il suppose, à tort, que noufar est un ancien mot ayant eu par luimème le sens de lotus, et qui est venu se souder à nîla, de sorte qu'on aurait dù dire à une certaine époque : le noufar bleu. Mais noufar n'est luimême qu'une corruption de ninoufar qui à son tour est pour niloufar.

DEVic dit à ce propos : «C'est probablement au médecin 0. Brunfels, mort en 1534, qu'on doit l'introduct. du mot nénuphar dans notre terminologie botanique. 》 Les botanistes européens ont employé ce mot longtemps auparavant, puisqu'il est donné par Sinos JANUENsis qui écrivait vers 1288 (Voy. Meter, Gesch. d. B.).; voici le passage de Simon selon Laxghavel: « nenufar arabicè dicitur nilofar. 》 D’ailleurs dès 1544, Duchesne, De stirpibus, l'employait couramment comme mot français (le nenuphar jaune, le nénuphar blanc).

2. Les feuilles du volet sont employées pour mettre sur le ventre ballonné. Deux-Sèvres, Souché, Croyances.

3. On dit de quelqu'un qui est très froid « qn'il a bu de l'eau de rolet», locution fondée sur la propriété antiaphrodisiaque du nénufar.

Centre de la France.

On dit à un jeune homme ardent auprès des filles : «pour te calmer on va te faire une infusion de ninfiaa. 》Semur en Auxois Côte-d'Or), c. par Il. H. Marlot.

«Le nom seul de nénuphar rappelle l'idée des propriétés sédatives, calmantes et surtout anti-aphrodisiaques attribuées à ce végétal; cette réputation, faite par les anciens, et qui ne doit peut-être son origine, ainsi que le remarque Marquis (Dict. des sc. méd. XXXV, 439), qu'à son habitation au milieu des eaux et à la blancheur virginale de ses fleurs (1), d'une odeur un peu nau-

(1) C'est surtout le nénufar blanc qui est employé en médecine, 
séabonde, est déjà établie dans Dioscoride et Pline (lib. XXV, c. 7), ete. Elle est arrivée jusqu’à nous et est devenue populaire; les poëtes et les naturalistes l'ont célébrée à l'envi; on s’est servi du nénuphar pour abattre les feux de la concupiscence; les pieux cénobites du désert en faisaient un usage fréquent; on en consommait beaucoup dans les cloitres, les courents, les séminaires, et on porta ses propriétés tempérantes si loin qu'on l'aceusa de rendre froid et même stérile. Les chanteur's en usaient pour conserver et perfectionner leur voix; on l'orlonnait pour guérir les insomnies érotiques, etc.

Cependant, les observateurs ayant remarqué que cetle racine abondait en fëcule; que les Tartares s'en nourrissaient, d'après Pallas, sans que cela muisìt à la fécondité de ceux qui en mangeaient; que sa saveur était un peu amère, visqueuse et styptique, ce qui indiquait des propriétés plutòt toniques ef irritantes qu'énervantes; que de plus, si on l'appliquait sur la peau, réduite en pulpe, elle y causait nne espèce le vésication. etc., ils furent portés non-seulement à douter des avantages de cette racine contre les aiguillons de la chair, mais i croire que ce destrncteur des plaisirs et ce poison de lannour, comme l'appelle Delille L'Homme des champs, chant 11I: en pouvait devenir le stimulant. Cette conjecture a été mise hors de doute par Desbois de Rochefort, qui a encore vil beaucoup employer le nénuphar dins les couvents de son temps, et qui effectivement, loin de voir agir comme réfrigérant, a observé que son administration était suivie de mauvais effets Mal. méd., II, (64). On peut également douter de la prétendue vertu hypnotique du nénuphar, siggralce par quelques auteurs et quaucune preuve directe ne vient confirmer. »)

Jínat, Dict. univers. de mal. médicale, 1830.

4. "Dic im Herbste gesammelten und getrockneten Blïter beider Seerosen werden in Rheinthal gegen Brand aufgelegt, wenn man sich $\%$. B gehauen oder gequetscht hat. ")

Suisse allemande, WintMan.. Tolksbol.

5. Quand le pluliuu nénufar sort de l'eau il n'y a plus de gelées ì - craindre.

Proverbe du Centre, Jaubert, Giloss.

1. Wird ein Wurzelstock unter das Bett gebracht, in welchem man schlaft, so ist man vor Krïmpfen gesichert.

Canton de Saint-Gall, IVartanas, Volkibol.

7. Sèbleter sind in der Fahne Herivîges von Sêlanden.

Gudrcx, 1373, 4 (Mloyen-Age illentand), BEXEcKe, Mittehochdeulsches Wverterb. 
8. - D'après des documents anciens, on constate que la fenille de nénuphar servait autrefois comme marque de cheval; elle était habituellement placée sur l'une des cuisses.

\section{Guenebault, Dict. iconograph., sulh verho} Marques de chevaux.

9. Les enfants enfoncent dans la partie supérieure du fruit du nénufar une petite baguette et s'amusent ì simuler l'action de baratter, d'où le nom de baratte donné au fruit. - lls s'en servent aussi, comme de jouet, en l'appelant bouteille, cruchon, etc.

\section{NYMPHAEA ALBA. (LINNÉ). - LE NÉNUFAR BLANG.}

Nons :

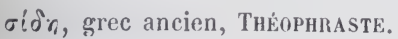

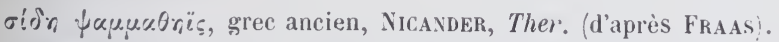

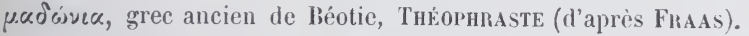

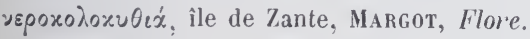

nenuphar album, nymphæa candirla, nymphæa alba, anc. nomenclat. nenufar fomina, anc. nomenclat., J. Cayus. Studio di lessicogr., p. 10. nenufar blanc, m., français anc. et morl.

blanc d'eau, m., anc. français, Les confections aromatiques, 1568, p. 396;

Guidon des apotiquaires. 1578, p. 396; Linocier, Hist. des plantes,

1584 ; Bauderon, Pharmacopée, 1655, p. 56.

plateau à fleur blanche, m., français, GEofFroY, Mat. médlc., 1750, t. I, 2 e sect., p. 202.

volet blanc, m., Anjou. - Poitou. - Maine.

canotte blanche, f., Aube, Drs Erangs, Toms pop.

blanque aplompe, f., anc. wallon, J. CAyrs, Un ms. namurois. (" oele de

blanques aplompes est très boin pour le foie qui est empiriez.» vaquevitériau, m., Valenciennes, HÉCART, Dict. rouchi. lreivakon, Villeneuve (Suisse romande), BRIDEL, Gloss. éli des étangs, m., Gard, comm. par M. P. FesQuet. pommes roses, Banvou (Orne), JoRET, Fl.

lunette d'eau, f., français dialectal en quel pays?), LAмARCK ET Candolle.

carfaro, carfaro femmina, copripentole, erculea bianca, ranocchiaj doppi, italien, TARGIoni, Diz. botanico.

rosa de amor, golfan blanco, espagnol.

golfão branco, portugais.

plamina alba, roumain, Ciнас, Dict. daco-rom. 
nufar alb, pluta, roumain, BRANDZA, Limba botanica, 1882.

plumine albe, roumain de Transylvanie, Fusz, Trivialn.

plumuna alba, plumiera, roumain de Transylvanie, Brandza, Limba botanica.

weisse seeblume, weisse nymphe, weisse seerose, allem.

seechrüseli, Oberland bernois, PRITz. et JEss.

gôske, buttbladen, Frise orientale, Focke, Pflanzennamen, 1870.

wesse göeskes (= petite oie blanche), Frise orientale, Pritzel et Jess.

sihblommen, all. de Transylvanie, Fusz, Trivialnamen.

hatschen, weisse mummel, Prusse occid., Treichel.

witte plompen, pannekoek, hollandais, Nevrich, Polygl. Lexicon.

buillite, irlandais, CaMeron. [H. G.].

curririn ban, duilleog-baite, colinih auhun, liagh loghar, irlandais,

Threlkeld. [H. G.].

alaw, magwyr wen (= mur blanc), lili ' $r$ divr, (= lys d'eau) gallois,

J. Davies, 1632. [H. G.].

duileay bhaite bhàn (= drowned white leaf), rabhagach (= giving caution or warning, a beacon), lili bhàn (= white lily), gaélique écoss., Cameron. [H. G.].

vodeni plutnjak, leken, lepen, lapoč, lokvanjača, luprik, omlak, kordon,

blatni cvit, vodeni podbio, tilivina, bared, jeserska lilija, berećna

trava, cviet mrtvi, serbo-croate, ŠULEK.

wóllia leluja, wende, Schulenburg, Wend. Volkisth.

housky $(=0 \mathrm{ie}$ ), bili hletchyli (cruches blanches), lapouchny vodiany,

bably (= petites vieilles femmes), petit russien, comm. par

M. Тн. Volkor.

wodnoi lapuschnik, russe, FaLk, Top. Kenntn. d. Russl.

lepei, lugmei, lithuanien, JACOBY, Lit. Pflanzennamen.

lelipes baltas, letton, Hupel.

fejér vizitök, magyar, Fusz, Trivialnamen.

lumme, lummet, pulpukka, walkia pulpukka, finnois, Fellmans, Index. lumme lehed, kuppo lehhed, esthonien, Fellman, Idem.

bejus nufar, turc, Sestini, Voy. dans la Grèce asiat., 1789.

bolbön jussur, kalmouk, Falk, Top. Kenntn. d. Russl..

FOLKLORE.

«Wenn man die weisse Mummeln in's Haus bringt, soll das Vieh sterben. 》

Prusse occidentale, Treichel, Poln. westpreuss. Vulgaernumen von Pflanzen (dans : Schriften d. naturforsch. Gesellsch. zu Danzig, V. Band). 


\section{NUPHAR LUTEUM. (SMITH). - LE NÉNUFAR JAUNE.}

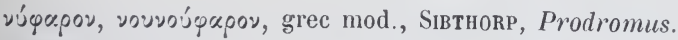

nутрhæa lutea, nymphæ citrina, anc. nomenclature, Bduнin, Pinax, 1671. nenuphar citrinum, anc. nomencl., Les confections aromatiques, 1568, p. 396.

nenufar mas, nomenclat. du XVIe siècle, J. Camus, Studio di lessicogr., p. 10.

nenufar jaune, m., français anc. et mod.

jaulne d'eau, m., jaune d'eau, m., anc. franç., Les confections aroma-

tiques, 1568, p. 396 ; Guidon des apotiquaires, 1578, p. 396 ; LiNocier, Hist. d. pl., 1584.

jaulnet d'eau, m., jaunet d'eau, m., anc. fr., BRоHоN, De stirp., 1511;

Cotgrave, Fr. d., 1650.

jonais, m., anc. wallon, J. CAMus, Un ms. namur.

jaunet d'eau, m., Normandie, JoRET, Fl.

jaunet des marais, m., anc. fr., Duez, Diz. ital. fr., 1678.

djanè, m., wallon, Feller.

volet jaune, m., Anjou. - Maine. - Poitou.

canotte jaune, f., Aube, Des Étangs, Noms des plantes.

ribarde, f., Anjou, Desvaux, Flore de l'Anjou.

aillaut d'eau, m., Oise, Graves, Catal., 1857.

amello, italien, Duez, Diz. ital. Fr., 1678.

carfaro maschio, erculea gialla, ranocchiaj gialli, zucchini da tabacco les

fruits), italien, Targioni, Diz.

cubiletes, esp., Colmeiro, Dicc. de los nombres, 1871.

golfan amarillo, esp., Lusitanus, Dinsc, medica materia, 1554.

golfão amarello, portugais, BRoTERo, Flora lusit.

nufar galben, roumain, BRANDZA, Limba bot.

gelbe seerose, allemand.

geele göeskes (petite oie jaune), Frise orientale, Pritzel et Jessen, Volksn.

gelbe mummel, Prusse occident., Treichel.

bwltws, gallois, J. Davies, 1632. [H. G.].

cabhan, abhain, irlandais, 0'REILLY. [H. G.].

lekuta, tchèque, Šulek, Jugosl. im. bilja.

lekuti, leknuti, lehnin, lekno, natkový koren, stulik, tasky, růze v'odni, tulipan vodni, tchèque, A. MüLler, Wört. syn. Nam. d. off. Pflanzen. 1866.

kijonka, kur‡a dupka (= Hühnerarsch, weil Frucht ihm gleich), polonais de la Prusse, Treichel, Poln. Vulgärnamen. 
blatuik, lekuta, žnti lokvanj, vodena ruža, bozur vodeni, želučnjak, rumeni plučnili, ¿̇uti lopoč, serbo-croate, Šulk, Jug. im. bil.

bi̊zoles, lithuanien, JacoBr, Lit. Pflanzenu.

wodolei, liufchimschiki, russe, Falk, Top. Kenuln. il. Russl.

jorté lataltia (= nénufar jaune), jovty hletcliyliy, zbanok (= petite cruche)

(le fruit), kapeliouchy (= chapeaux) (les feuilles), toustoun ( $=$ le

gros) (la racine) petit russien, e. par M. Tн. Volkov.

lehpes ilseltanas, letton, HUPEL.

sari mufar, turc, Sestini, Voy. dans la Grèce asint.

pufer ciceghi, ture, sibthorp, Prodromus.

lhuouzân (esp. de nénuphar à fleur jaune et ì bonne odeur), arahe, Frevtag.

lummet, lumme, pulpuklia, leltaineu pulpulika, finnois, Feldman, Inde.1 pl., 1835.

tombojuk, bachkire, Fellmax, Idem.

liolijet kuppo lehhed, esthonien, Felluax, Id.

2. Dic Tïrken bereiten aus den wolriechenden Blïten ein kïhlendes Getränk, das sie Pufer cicegli nennen. (ll s'agit d'une variétí à bonne odeur).

liosenthal, synopsis plant, diaph.

\section{MYMPIILA LOTUS. (LINNÉ).}

i.

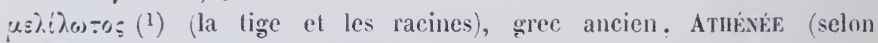

Mever, Geschichle d. Botanik).

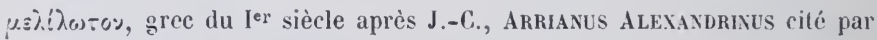

Mever, Gesch. d. Botanik).

lotier d'E!lypte, m., lotus des auciens, m., français savant.

lodio do Egypto, portugais, Nemnich, Polygl. Lexicon.

noufar, buclienyn el kilianz!yr, a'râys el Nil (id est : uxores Nili), arabe

égyptien, Desule, Flora aegypt. illustr., 1824.

Sur les noms que les anciens Égyptiens donnaient aux différentes espèces de Nymphaea, voyez :

Victor Loret, Sur les noms égyptieus du lotus (dans: Recueil des tra.

l'aux relatifs à la philologie égyptienne, Paris, 1870, p. 190-196).

Raffenead-Delile, Observations sur les lotus d'Egypte, (dans Aunales du

Museum d'histoire naturelle, t. I, 1802, p. 372 ì p. 382.

(1) C'est le produit alimentaire de la plante. Voyez: Salmasius, Exercitat. plinianae, p. 687 . 


\section{NYMPHAEA GERLLEA. (SAVIgNy).}

รวบัร0\% ('), grec ancien, d'après un manuscrit du XVe siècle, E. FournIER,

Réforme de la nomenclat. bot., 1880, p. 7 (tirage à part du Journal des savants).

uymphea bleu d'Egypte, m., français.

bachenyn, a'rûys el Nyl, byâroù (la racine), arabe égyptien, DeliLe, Florae aegypt. illustratio.

Pour les noms arabes de cette plante, voyez: Ibs Beithar, Les simples, édit. Leclerc, II, g29.

\section{NYUPHAEA CIANEA. (RoxBUrgh).}

\section{1. - NOMS :}

nénuphar bleu, m., nénuphar bleu des Indes, m., lotus bleu, m., français. milotpala $\left(^{2}\right)$, asitotpula, indierara, mṛ̂ûtpala, sanscrit, BoEHTLINGK, Sanser. Wört.

2. Cette jolie fleur bleue joue un grand rôle dans la poésie sanscrite. Les poètes lui comparent, à chaque instant, les yeux de leurs belles.

\section{NYMPHAEA RUBRA. (RoxbURG).}

krschnakanda, raktotpala, sanscrit, BOEHTLINGK.

NELU.MBIUM SI'ECIOSL'M. (WILLDENOW.). LE NÉLUMBO.

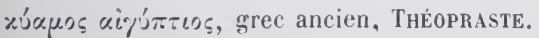

жoخoxaris (la racine comestible) $3 \%$, grec ancien.

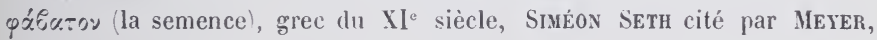

Gesch. d. Bot.

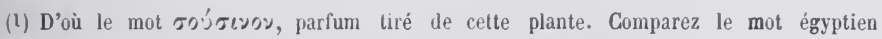
seschen qui sert à désigner une espèce de lotus, d'après LoRET, Rec. de trav, relat. à l'archéol. égypt., I've année.

(2) Mot à mot, lotus bleu; nîla bleu et utpala lotus en général.

(3) On a donné aussi ce nom grec à une plante d'une toute autre famille, à l'A rrum aegyptium (Colocasia antiquorum). 
nenufar purpureum, nomencl. du XV $\mathrm{XV}^{\mathrm{e}}$ s., Casus, L'opera salern., p. 95. nymphaea nelumbo, nomencl, de LiNsé.

faba æyyptiaca, faba æryjptia, anc. nomencl.

febve egyptiaque, français, HuLoet, 1572.

nélumbo, m., nélombo, fève d'Eyypte, français.

padma, bisinî, bisanâbhi, radjîinî, cuṇ!̣a, nalini, panliadjinî, ambudja,

aravinda, sanscrit, BoEHtLingk, Sanscr. Wört.

bakilla kobty, fübés el libty, djâmissa, arabe, Iвм Beïthan, Eı. Leclerc. qoulquis, foul massrî, baqala massrî, arabe syrien, BERGGREN.

salabagala (les fruits), Perse, Guelin cité par Nemnich, Polygl. Le.x. nelun, singalais, WATSON.

liorkansi, copte, DE SAcr, Abd Allatif, p. 98.

2. Cette plante se fait remarquer par de magnifiques fleurs roses odorantes; elle croissait autrefois dans le Nil, mais depuis longtemps elle en a disparu, et ne se voit plus que dans l'Inde, à la Chine, aux Moluques, au Malabar, en Perse, en Arménie, etc. Son fruit, souvent figuré sur les monuments égyptiens, comparé tort exactement à un guêpier, ( $\left.{ }^{1}\right)$ par Théoplıraste, a le volume d'une pomme d'arrosoir et présente une multitude de loges parallèles et sur un mème plan, qui contiennent chacune une semence de la grosseur d'une noisette; ce qui les fait appeler noix on fèves d'Eggypte, faba segyptiaca, fèves pontiques, etc. ; en Perse on en fait des chapelets, etc. Ces semences sont comestibles et dans l'antiquité la plus reculće elles servaient d'aliment; en Egypte, on en faisait du pain, etc.

MÉRat, Dict. de matière médicale, 1830.

\section{PAPAVÉRAGÉES}

\section{PAPAVER RHEAS. (LiNNÉ). - LE GOQUELIGOT.}

\section{1. - NoMs DE CETTE PLANTE}

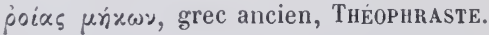

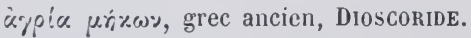

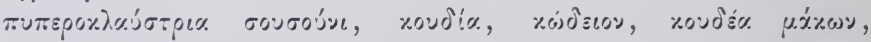

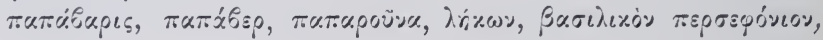

(1) En japonais, cette plante s'appelle fatsisou, c. à d. le guêpier. HoffmanN. 


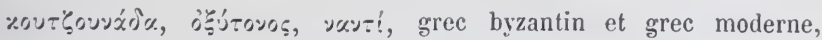

Laigkavel (1), Beitr. z. Gesch. d. Bot.

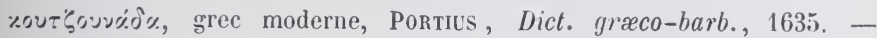

Germano, Voc. ital. greco, 1622. - grec mod. de Crète, Rallin.

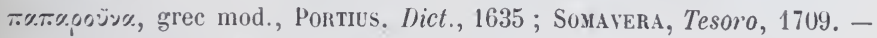

Ile de Zante, Margot, Flore de Zante.

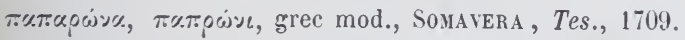

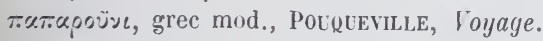

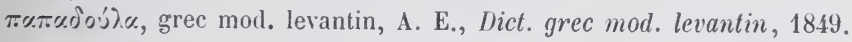

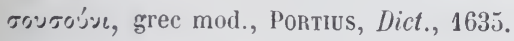

Tะระเวก́s, île de Chypre, Unger, Die Insel Cypern. 1865.

rubiola, latin du Moyen-Age, Diefenbacir, Gloss.

papaver rubeum, latin du Moyen-Age, Mowat, Alphita; J. Canus, L'opera salernit.

rosa silvatica, lat. du XVe s., J. CAMus, L'op. salern., p. 134.

anomon, lat. du moy. âge, Matthaeus Silvaticus, cité par Mowat.

amomei, papaver agreste, anc. nomencl., Synonyma (Suppl. de Mesue), 1623.

papaver erraticum majus, papaver erraticum, papacer rubrum, anc.

nomenclat., Bachis, Pinax, 1671.

papaver fluidum, anc. nomencl., Fuchsius, 1546.

$\operatorname{coq}\left({ }^{2}\right) \mathrm{m}$. , Normandie, Joret; DECorde, Dict. du pat. - Guernesey,

Mḱtivier, Dict. f. n. - Orne, rec. personnell.

co, m., Yonne, m., Jossier, Dict. - Eure-et-Loir, rec. personn.

coquelicoc, m., coquelicoq, m., quoquelicoq, m., ancien franç., Вroноs,

De stirpibus, 1541 ; Duchesne, De stirpibus, 1544; Cotgrave French

dict., 1650 ; P0NEY, Indiculus univers. 1667, p. 49. - L. LIGER, Le

jardinier fleuriste, 1718 , t. I, p. 142.

coquelicot, $m$. (le $t$ final ne se prononce pas), anc. fr., Pinaeus, Historia

plantarum, 1561; J. CAmus, Livre d'heures. - français mod.

coquelikeu, m. Bourg (Haute-Marne), rec. pers.

coquericoq, anc. franç., J. GRÉvis, Deux livres des venins, 1568, p. 252 ;

J. VictoR, Tesoro, 1609.

coquerico, m., anc. fr., L. RICHER, L'Ovide bouffon, 1662, p. 587.

(1) LANGKavel donne ces noms comme s'appliquant au genre papaver en général, n'ayant pu, sans doute, distinguer ceux qui signifient pavot de ceux qui ont le sens de coquelicot.

(2) La fleur rouge du coquelicot ressemble assez à la crête d'un coq dans les blés. 
coquerioco, m., anc. frans., Miroir général pour sacoir la lanyue espagnole, Rouen, 1623 , p. 376.

cocriacot, m., picard, Corblet, Gloss.

cokikó, m., Audincourt (Doubs), rec. personnell.

cottkolinkó, m., Tanville (Orne), rec. personnell.

cocalincot, m., Alençon, Dubors et Travers, Dict.

kélinkó, m., Manche, comm. par M. J. Courare du Parc.

cocolico, calicocô, calicô, calcalico, carcalico, carcalinco, carcarico, caricoco, carquélico, quercolico, querquélinco, coquelinco, cotelico, cotecolico, cotecolinco, coricoco, coqueriocô, Normandıe, JoREt, Flore pop. de la Norm. (Pour les localités où ces noms sont employés, voir l'ouvrage de M. JoREt.)

carcarico, Vimoutiers (Orne), Letace.

coquelicaou. m., Lssel (Corrèze), G. de Lépinar, Noms pat. des pl.

cocolico, concoudesco, f., concouresco, f., sud-ouest du Langued., Duboul. coquelicon, m., anc. franc., Cotgrave, French diction., 1650.

coquelincan, m., Jura, Moxiser, Vocab., 1823-1824, s. vo simbu.

coquelija, m., Saales Vosges, H.illant, Flore pop. vosg.

kilirijac, m., Raon l'Etape, Vosges), Haillaxt, Fl. pop. v'osg.

coquelijô, m., île de Ré, comm. par E. LEMARIÉ.

cacalijô, m., Rémilly, Pays messin, rec. pers.

coco!ijô, m., Woippy Pays messin). rec. pers.

catecalinjô. m., arrondissement de Nancy, L. AdAM, Patois lorr.

cocolicot, m. Haute-Bretagne, P. SÉBıLот, Trad. de la Haute-Bretagne, II, 334 .

terlicoco, m., Valenciennes, HÉcart, Dict. rouchi.

cacuraca, m., provens. mod., Garcis, Nouv. dict.prov. - Hérault, Loret,

Flore de l'Hérault. - Moyen Dauphiné, Moutier.

cacalaca, m., Hérault, LoRET.

cocolico, f., Aveyron, VArssier. - Tarn-et-Garonne, LAgRèze-Fossat. coquet, m., Vexin (Normandie), JoRET.

coquelet, m., Environs de Cambrai, Boxiface, Hist. d'Esne, 1863. -

Saint-Pol Pas-de-Cal.), c. par M. Ed. Edvost.

coquelourde, f., anc. franç., Duchesne, De stirpibus, 154.

galle, Nice, Colla.

jole, m.. Raon l'Etape (Vosges), Haillant, Fl. p.

poulas, m., Bessy (Yonne), Jossier, Dict., 1882.

poulogno, f., Alpes-IIarilimes, Mistral.

piéoulas, m., Aveyron, VAYssier.

jaline, f., Allier, comm. par M. C. Bourgougron.

poulo-gaou, m., gaou-galino, f., provenç. mod., Mistral. 
gacu-galin, (I) m., = coq-poule ), Apt (Vaucluse), Coligxon. - Env. d'Arignon, Palux. - Saint-Remy (Bouches-du-Rhône), M. Girard. Lis aupiho, 1878, p. 33. - Forcalquier, comm. par I. E. PLAuchud. galingalaou, m., (= poule-coq), prov. mod., CastoR, L'interprète prov. - Apt (Vaucluse', Colignox, Flore d'Apt.

gueringuingaou, m., provenç. mod.. CastoR, L'int. prou. ; Garcjs, Mour. dict. pr. - Forcalquier, comm. par II. E. PLauchid.

jaou-poula, Bas-Dauphiné, Movtier. rouget, m., Trévières Bessin), JoRET, Fl. p. rougerole, f., anc. franç., Mowat. Alphita. rouglelo, f., Lauragais, 1. Fotrès, Les grilhs, 1888, p. 84. - Castelnaudary, L'Alliance latine, nov. 1878, p. 70.

rouzela, f., Gard, Poczolz, Flore. 1857.

rouzelo, f., Tarn, GART, Dict., 1845. - Tarn-et-Garonne, LAGRÉZe-Fossat, Flore. - Castres, Covzinié, Dict., 1847. - Gard, comm. par M. P. Fesqlet. - Carcassonne, Elsèbe de Salles, Poésies, 1865, p. 335.

- Hérault, Barthès, Gloss, bot., 1873. - Toulouse, Tocrixon. Agenais, c. par M. l'abbé L. DARdy.

raouzèle, f., Libourne (Gironde), c. par II. Duraxd-DÉgraxge.

raouะèlo, f., Agen, rec. pers.

raousèro, gascon, c. par M. l'abbé L. DARuY.

arrouzèlo, f., Lectoure, c. par M. J. F. BLADE. - Gers, c. par I. H. Daignestous.

risolo, f., Livron (Dròme), c. par MI. E. H. SIBotrg.

rijôla, f., Lyonnais, Putspelt, Dict., 1888. - Gilhoc Ardèche), ClugNet, Gloss. du pat. de G. 1883.

rousouol, m., Aveyron, VAYssier, Dict. put.

rousoula, f., moyen Dauphiné, Moutier.

ronzula, f. rusula, f., Haut-Dauphiné, Мoutier.

rouelo, f., provenç. mod., Garcis. N. dict. pr.; Castor, L'interpr. prov.

- Apt, Colignow, Flore d'Apt. - languedocien, c. par II. H. Fat. planto rouelo f., Apt, Coligxon.

planto roalo. f., environ d'Aix, GARIDEL.

ruelo, f., Toulon, Patout, Pluntes médic., 1864. - Var, Haxri, Catal. -

Bouches-du-Rh., Villexeure, Statist.

riuelo, f., environs d'Avignon, Palun, Catal.

rouelle, f., Aix en Prov., Boyer de Fonscolonif, Calendrier, 1845.

roelle, Pyrénées-0rientales, Companyo, Hist. nat., 1864.

(1. Pour l'explication de ce mot, voyez ci-dessous le jeu enfantin de poule ou coq. 
roouro, f., Forcalquier, comm. par M. E. Plauchud.

rourella, f., Nice, Colla.

vermallons, Pyrénées-0rient., Companyo.

popelure ( $\left.{ }^{\mathrm{I}}\right)$, f., anc. franç., P. MEIER, Deuxième rapport sur une mission, 1867.

roso, f., canton d'Espalion (Aveyron), rec. per's.

rose de loup, f., Centre, Jaubert, Glossaire.

canroso. f., Montauban, Gaterau, Descript.

canrojo, f., Lot, comm. par M. J. DAYMARD.

conroso, f., Averron, VArssier, l)ict.

cap roso, f., Tarn-et-Garonne, LAGrèze-Fossat, Fl. - langueducien,

Duboul. (M. Duboul traduit cap roso par tête rouge).

canréso, f., Tarı-et-Garonne, Avr. PAgès, Cot' de floitos, Paris, s. d., In-8, p. 196.

passo rosos, Gers, Dralet, Topoyr. du Gers.

passe-rose, Vic-Bilh (Béarn), LEspy, Dict. béarn.

fiame de feu, f., flamme de feu), Bru (Vosges), Halliaxt, Fl. pop.

feu d'enfer, m., Haute-Marne, comm. par M. A. Dagun.

feu d'enfar, m., Forêt de Clairvaux (Aube), Batdous, Gloss., 1887.

feu d'infa, m., Jura, Mosien.

feuy' d'enfer, bouquet du diable, m., Soulaucourt (Haute-Marne), c. par II. l'abbé MarchaL.

chaudière d'enfer', f., Haute-Marne, comm. par II. A. Digur. - Langres, Mulson, Vocab. langrois.

chaudrotte, f., Villers-le-Sec (Haute-Saône), rec. pers.

tsôdère (= chaudière), f., fu d'anfa, m., Canton d'Arbois (Jura), c. par M. L. DORBON.

mahhon brelee, f., (= maison brûléc), Courbessaux (arrondiss. de Lunćville), L. ADAM, Pat. lorr.

môhon breulèie, f., móhon breulèye, f., Romont Vosges), Charmes (Vosgges),

Haillant, $\mathrm{Fl}$. pop.

môhon breulaye, f., Gircourt (Vosges), IDEM, ibid.

lumé, m., Hautes-Alpes, Jouglard, Mols du patois des Hautes-Alpes.

fió volage, m. (= feu sauvage), Thiers (Puy-de-Dôme), rec. personn.

feu sauvage, m., Haute-Bretagne, P. SÉBillot, Tradit. de la HauteBretagne, II, 334.

(1) Ce mot se rattache sans doute à pourpre. - En Lorraine, la maladie appelée en français rougeole, porte le nom de propelieure, popelieure 
confanon, (1), m., anc. franç., Pinaeus, 1561; - Duez, 1664; - Du Pinet, 1660.

confaron, m., Lons-le-Saunier, DarToIs, Etude des pat., 1850.

confoiron, m., Montbéliard, Contejean, Gloss.

paparri, prov. mod., RÉGuIs.

pavot, m., Châtenay (Seine-et-Marne), rec. pers. - Mayenne, comm. par un botaniste de la Mayenne. - Clamecy (Nièvre), rec. pers.

pavot sauvarje, ponceau, anc. fr., Du PINET, 1660.

pavot sauvaige, m., pavot des champs, m., anc. français, Camus, L'opera salern., p. 101.

pavot rouge m., anc. franc., J. Camus, Live d'heures. - Duchesne, De stirpibus, 1544. - Fillassier, 1791.

pavot roudzou, m., poutarousa, f., Vallorbes (Suisse rom.), VAILotTon-

AubEit, Vallorbes, etc. 1875.

pavot batard, m., Combloux (Haute-Savoie, rec. pers.

pavot de bled, m., pavot des bleds, m., anc. franç., J. Victor, Tesori,

1609 ; P. Morin, Rem. sur la culture des fleurs, 1674, p. 99; 0udin,

Trésor, 1660.

papou, m., Centre, Jaubert, Gloss. du Centre.

paou, m., Saint-Maurice (Haute-Savoie), rec. pers.

pavou, m., Allondaz (Savoie), rec. pers. - Canton d'Aime (Savoie), c. par

M. Marjollet.

pabou, m., Saintonge. - Poitou. - Anjou.

pabot, m., Loire-Infér., Alc. Leroux, Patois actuel de la Mée, 1886.

pobót, m., Saint-Santin-Cantalès (Cantal, comm. par M. J. MaLveziN.

paveux, m., Plancher les Mines (Haute-Saùne), Poillet, Vocab. - Nor-

mandie, JoRET.

pitit pavoir, m., Verviers, SEMERTIER.

pavoir, m., Verviers, Lejeune, Flore. - Ardenne belge, A. Bodr, Vocab.

des agricult. - Spa, LEZAACK, Dict. des noms wallons.

pavoèr, m., Ardenne belge, A. BoDr.

pavôr, m., paouêr, m., rotch paouê??, m., pti paouêr, m., wallon, FELLER. pavas, Bessin, JoRet.

pan, m., (écrit quelquefois à tort paon), disne, BrAYER, Statist. de l'Aisne.

- Environs de Soissons, rec. pers. - Canton d'Estrées Saint-Denis

(Oise), Précis statist. sur le canton d'Estrées, etc., 1832, p. 75. 0ise, Primes d'honneur en 1869; Paris, 18\%2, p. 64. - Pays de Caux, JoRET, Fl. $p$.

(1) Le mot confanon en vieux français avait le sens d'étendart. 
pouencel, m., poonciau., anc. fr., Zeitsch. f. rom. philol., 1880, p. 371. pouncel, m., frans. du XII ${ }^{\circ}$ siècle, Biblioth. de l'Ecole des Chartes, 1869, p. 328; GodefroId, Dict. povencel, m., anc. frais., Romania, 1881, p. 30.

ponceau, (l) m., anc. français. - Centre de la France. - Maine. - Anjou. - Aisne. - Oise.

ponciau, m., Environs de Paris. -- Loiret. - Eure-et-Loir. - Berry. panciau, m., Centre de la France, Jalbert. - Berry, Litrné. Dict. Allier, conım. par M. E. OLIVIER.

ponchet, m., Pays de Bray, DECoRDE.

panchet, in., pancet, m., poinceau, m., Oise, Graves, Ciatul. des pl.

penchot, m., penchet, m., poinçot, m., Oise, Primes d'honneur en 1869 ,

Paris, 1872, p. 64.

poinciot, m., Chaumont en Vexin, Friox, Descript. de Ch., 1867.

ponchereux, m., Pont-Audemer (Eure), Rosis, Dict. - Normande, Joret, Flore pop.

poncereux, panchereux, poncheleux, ponchereau, pancheron, poincet, poinciau, poinçon, Normandie, JoRET.

mahon, m., picard, Corblet, Gloss. - normand, Delroulle, Gloss. -

Oise, Graves, Catal. - Aisme, Brayer, Statist. de l'Aisne. - Pas-de-

Calais, comm. par M. Ed. Edmont el M. B. de.lierhervi. - ( En

Picardie le garde champêtre est généralement appelé d'une fa̧on

burlesque : garde-mahon. 》 Comm. par Ed. EDsost.

moine, m., Anjou, Desvalx, Flore de l'Anjou.

ymillame, m., Montbéliard, Coxtejean, Gloss.

fantina, f., mentonais, Axdrews, locab.

papı, m., Bessin, JoRer, fl. pop.

papitre, Lieuvin, Plaine de Caen, Bocage (Normandie, JoREt, Fl.p.

popitre, Evrecy près de Caen, rec. pers.

poupi, Pays d'Auge et Plaine de Caen, JoRET.

boubi, boubon, bourbiton, Normandie, Joret.

babi, m., Nice, Risso, Hist. nat., $18 \supseteq 6$.

flour dé babi, f., flour dis anyes, ( ${ }^{2}$, f., prov. mod., Mistral, Tresor d. fel. babou, m., Ineuil (Cher), rec. pers. - Berry, J. TIssier, Dict. berrichon.

(1) Pour l'étymologie de ponceau, voyez Romania, 1881, p. 302 et Zeitsch. f. rom. Philol., 1880, p. 374-375.

(2) Dans diverses localités de la Provence, de petıts enfants, habillés en anges, répandent ces fleurs devant le Saint-Sacrement, à la procession de la Fête-Dieu, d'où le nom fleur des anges. 
babeau, m., Allier, comm. par M. E. OLIVIER.

tsignosse, f., Montaigu-le-Blin (Allier), c. par M. F. Duchos DE LA Jarousse. - Dépouillée de ses pétales la fleur ressemble à une tignasse).

chenute, f., Centre, Jaubert, Gloss.

d'chonatte, f., Ban de la Roche, H. G. OBErlis, Descript., 1806.

chanotte, f., chenatte, f., savège chenatte, f., Vosges, HaIllant, Fl. p. coucou, m., Valenciennes, HÉcart, Dict. rouchi. - Environs de Cambrai et. de Cateau-Cambrésis (Nord, rec. pers. - Romorantin (Loir-etCher), rec. personi.

ékerpelle, f., Béthune (Picardie), Conblet, Gloss.

cabosseta, f., Suisse romande, VICAT, Hist. des plantes vénéneuses.

ander, Brive (Corrèze), G. de LépiNay, Noms pat.

onder, canton de Saint-Germain (Lot), Soulié, Catal., 1883.

graouzela $\left({ }^{\mathrm{l}}\right)$, f., Montpellier, LoRet.

graouzelo, f., Toulouse, AzAïs, Dict. des idiomes langued.

yrajan, m., Allier, comm. par M. E. OLIVIER.

grô-dzaï, m. = gros coq), Paulhaguet (Haute-Loire), comm. par M. P.

LE BLANC.

grèjó, m., Issoire (Puy-de-Dôme), comm. par I. J. BAREIRE.

pied $d$ ' co, m., canton de Périers (Manche), rec. pers. - Saint-Germain-

sur-Ay (Cotentin), Jorft, Fl. pop.

maou d'uy, env. d'Aix, GARIDEL.

maou d'iu (= mal dyeux), Arles, LAvgier de Chartrotse, Nomenclat.

patoise. - Environs d'Avignon, Palun, Cat.

lagagne, f., languedocien, Laukent Joubert, Erreurs pop., 1579, p. 288.

plantiau, m., Orne, L. Duval, Enquête.

trounicassés, m. pl., sud-onest du Languedoc, Deboul.

fleûr di tonir, f., Liège, Forir, Dict. - wallon, FELLER.

tonire, tonoire, wallon, GraNdgagnage, Dict.

fleur du toni, pti solo, m., (= petit soleil), colo, m., colo, m., wallon, Feller.

tonouêr, Namur, c. par II. 0. CoLso心.

passaouec, m., Luchon (Pyrénées), Jul. SACAZE.

vamôque, mauvo, piône, Norm., Joret.

môvièto, f., env. de Vảlence (Drôme), rec. pers.

parpèl, m., Gard, comm. par M. P. Fesquet.

flour de serp, f., Areyron, VAyssier, Dict.

(1) LORET dit que ce nom est donné au coquelicot avant la floraison. 
toulipo rougeo, f., lepègue, f., Aveyron, VAyssier.

tulipan, Forcalquier, comm. par M. E. Plauchud.

pamaau, painaau des champs, Vosges, Halllant.

clacoutère, f., Landes, c. par M. l'abbé T. Forx.

clacote, f., Labouheyre, Landes, c. par M. F. ARnaudin.

marque, (1) f., Malmédy (Pays wallon), Feller.

papavero, papavero selvaggio, italien.

rosolaccio, ital., C.Esalpinus, De plantis, 1583.

bambagelle, bubboline, citole, scitole, rosillaccio, pastriccia, italien, TARGIONI, Diz.

papavero sercian, Vérone, Pollini, Fl. ver.

serchione, Vérone, SÉGuier, Plantæ veronenses, 1745.

papaver, Mont-Cenis, Re, Flora segus. - piémontais, Colla.

papaiveo sarvaego, Gênes, Casaccia, Diz.

panpavero, Rovigno (Istrie), A. Ire, Canti popol. istr., 1877, p. 314.

papà'ar, confenón, Frioul, Pirona, Voc.

papavar, confalon, Tessin, Pollini, Fl. ver.

popolana, pompola, lombard, Scannagatti, Erbe, 1794.

popolanna, milanais, BANFI, Voc. mil.

papavêr sâlvâdag, cônfanon, Plaisance, Bracciforti, filora piac.

papata, Fabriano, Marco.sldi, Guida della ciltà di Fabriano, 1877.

pappata rossa, env. de Pérouse, Zanetti, p. 238.

rosola, environs de Padoue, patriarchi.

rosolina, Trévise, Saccando, Flora trev.

fantine, Corse, Primes d'honneur en 1865, Paris, 1876, p. 464.

madonina, Brescia, Melchioni, Voc. bresc.

madonine, rosolie, madoi, Brescia, ZERSI.

donetta, Monferrat, G. Ferraro, Bot. pop. (Archivio d. trad. pop., 1885, p. 407).

madonna, Abruzzes, lDEM, ibid.

madone, madonne, madöne, Piémont, BaLbIS, Piante dei contorni di Torino; Zalli, Diz.; CaPello, Dictionn.

done roselle, environs d'Asti, Camisola, Flora astesa. [J. C.].

rusele, Casal (Piém.), Colla.

basadone, Alba près Cuneo (Piém.), Colla.

(1) Ce nom vient de la marque que les enfants se font sur le front en y faisant éclater les pétales pour faire du liruit, voici le procédé : on cucille un pétale, on en rapproclie habilement les borús en forme de sac, puis on fait éclater ce sac sur le front ou sur la main, arec le plus de bruit possible. Feller. 
belledon, C'ossano (Piém.', Colla.

pè d' done, Annone (Piém.), Colla.

lanadone, Oviglio et Agliano (Piém.), Colla.

paradun, San Vittoria (Piém.), Colla.

done donëtte, donëtte russe, Alexandrie, Cunlo, Mondovi (Piém.), Colla. pitadoni, Asti Piém.), Colla.

pol, poli, Tortona et San Griuliano Piém.), Coll.1.

basadöne, piémontais, ZALLI, Dizion.

papayno, napolitain, Puoti; Gusumpaur.

papagno selvaggjo, Vésuve, Pasquale, Fl. ves.

papanela, Morano et Castelleti-Merli Piém.), CoLl.1.

pupör, pupöl, Vogglıera et Castelnuovo-Scrivia (Piém. ', Colla.

surcure, San Stefano Belbo (Piém.), Colla.

paparina, Calabre citérieure, Donsa, Tradizione nei dial. d. Culabria. -

Sicile, Macaluso, Voc.; Branca, Flor'a d'A'ola.

paparina, scattarole les fleurs', Matera, GATtixi.

paparina russa, sicilien, LAgess, Erbuario, 17亿二.

paparina sarvaggia, sicilien, Cupaxi, Ilortus cath., 1696; Mortild.ro.

pabauli, sarde méridional, Spaxo

pappaille, m., pabaile, pubuza, sisia, f., sarde logodourien, SpAvo.

pabaúli arrubuu, Sardaigne du suil, MoRIs, Flora sarl.

papaveru arribiu, Sardaigne du norl, IDE.I.

papariu arrubiu, Alghero (Sarlaigne), InE.I.

papaosu, Flumini Major (Sardaigue), IDEM.

fiuron d' gran, mson d' gran, romagnol, MoRri.

squácquera ('), Barletta, Bruxi.

yarusola, marusola, yaroeusola, marusoela, Mantoue, Cherubini.

quicaracoq, Calella (Catalogue), Cixi, Flora de Cialeltix.

cararequecs, Minorque, Costa, Flor'a de Catal.

rossella, rosella, catalan anc. et mod. - valencien, Ros, Dicc. valenc. 1739.

ruella, catal., Bull. de ass. d'cxcurs. catal., 1890, p. 134.

roella, Baléares, Marís, Catal., 1880 ; Figuera.

paramú, gallarets, Vall de Nuria Catal.), VAYREDA, Flora, 1882.

hamapola, amapolu, abuból, abriba, papoila, (2) espagnol.

(1) Nel linguaggio popolare squàcquera significa cosa molto aperta. La corolla di questa specie è assai distesa. (Bruni).

(2) Ces formes viemnent prohablement, selon Dozy, Gloss., de l'arabe hhabba baura signifiant graine de jachère, nom du coquelicot. 
amapola, yanapola, Sud de l'Espagne, Borssier, Voy. dans le midi de l'Esp.

ababol, aragonais, Borio.

mapola, galicien, Cuverro ; Valladares.

papoula, papoila, portugais.

papoileira, portugais, Nemnich, Polygl. Lexic.

mac roshu, paparóne, paparuna, roumain, Branda, Limba bot.

mac silbatic, roumain, Cıнас, Dict. d. $r$.

wiltmayo, anc. allem., Dierenbach, Glossar.

rotman, roceman, anc. haut all., PRitzel et Jess., Volksnam.

veltmage, ylatzen, appellon, appelrot, oppfelbuet, chlafferbluem, lilappel,

klapperblume, moyen haut all., Pritzel et Jessen.

klitsche, moy. haut all., Grimm, Wörterb.

klapprose, klatschrose, flitschrose, kornmohn, kornblume, rothe korn-

blume, ackiermohn, feldmohn, feldmaysame, allemand.

kat:enmagen, himschall, fallblume, blotze, allemand, Nемхісн, Polygl.

Lex.

klutsche, plapperblume, allem., Grimi, Wört.

klapperrose, liornrose, willtmaen, allem.. CoRdus, Botanologicon, 1533.

flietschrosen, klietschrosen. grindmagen, schnallen, allemand, G. R.

BOEHNer, Commentationes, p. 52.

fallblume, allemand, GRimi, Wört.

lilatschmohn, Prusse, Frischier, preuss. Wört.

herrmolın, Prusse, Treichel, Vollisthüml aus der Pflan:enwelt.

lilitscheblume, Henneberg. SpIEss, Beitr. zu einem Henneb. Idiotikon,

1881.

bersthraut, boschliraut, paterblume, Eifel, Wintgen, Vegetat. d. Eifel.

engelsblum, engelskap, kokliko, m., Luxembourg, J. WEBER.

klapprause, Westphalie, LANDoIs, Die westfäl. Pflanzenn.

blatzblumen, Thuringe, Pritzel.

gulle, Wurtemberg, IDEM.

muech, kokeschblommen, rüht kiuhreblommen. allemand de Transylvanie,

Fusz, Trivialnamen.

lolrôse, Gøttingue, Sснамвасн.

maluflie, Silésie, Pritzel.

klaotschen, fürblöm, smok, sud de l'Altmark, DanNeIL.

fakel, f., bläse, f., Souabe, Sснмn .

billar maghen, allem. des Alpes vénit., SCHMELLER.

maon, Altmark, Pritzel et Jessen.

schnalle, Memmingen, IDEM.

schneller, schnellblumen, Iugsbourg, IDEM. 
rollabluoma, flügabluama, flätterrosa, fürbluama, canton de Saint-Gall. Wartmann, Volksbotanik.

jungfernkraut, Suisse allemande, Durнeim, Schw. Pflanzen-Idiot.

stinkrosa, huetrosa, canton d'Appenzell, IDEM.

purpalizn, purperlitzen, Carinthie, Zwayzıger, Verzeiclin.

pfaffaros'n, pfaffataschl, hlatschros'n, feldmayn, droad magn, Basse Autriche, HöFER, Wört., 1884.

wilde heul, klapperroos, kollebloem, ancien flamand, DoDoxaEus, CruydtBoeck, [A. DE C.].

kollebloem, klapperroos, klaproos, koornheul, wilde heul, kankerbloem, korenroos, roode korenbloem, hollandais. [A. DE C.]

spokelbloeme, Frise, Waling Dikstra. [A. DE C.].

gokelbloem, anc. flamand, DE Bo, Idiot. [A. DE C.].

bloedzuipersbloem, bloedzuigersbloem, honilsrooze, kankerbloem, roozevied, wulvebloem, flamand, DE Bo, Idiotik. [A. DE C.].

bloedzuipers, bloedzuigers, Brabant, Is. TeIRlinck, Plantlore, I. [A. DE C.] popig (1), baso popig, anglo-saxou, Cockarne, Leechdoms, 1866.

papoeg, popaeg, anglo-saxon, DiefenBach, Gloss.

wild poppy, anc. angl., Mınsheu, Dict., 1623.

lightning, Devonshire et Berkshire, Friend, Gloss. of Dev.

redweed, East Norfolk, SkEaT, Reprint Glossaries, 1873.

ridweed, île de Wight, Lovg, Wight dial.

cuprose, Yorkshire, Marshald, Rural Economy, 1796.

copperrose, est de l'Anglet., Forвх, Voc. of East-Auglia.

cankerrose, est de l'Anglet., IDEM.

headwark, nord de l'Anglet., Engl. dial. soc., 1874, p. 38.

headache, est de l'Anglet., ForBx, Voc. of East-Anglia.

thunderbowt, Shropshire, JAckson, Shropsh. Word-book. - South

Cheshire, Darlington, Folk-speech.

thunderbolts, anglais dialectal, Notes and Queries, 2 oct. 1880.

ear-aches, anglais dialectal, Britten, Plant-names.

blind eyes, idem, IDEM.

blindy buffs, idem, IDEM.

corn rose, idem, IDEM.

corn poppy, red poppy, idem, IDE.I.

cock's head, idem, IDEм.

cock's comb, idem, IDEM.

(1) Ce mot signifie poupée; les enfants font de très jolies poupées avec les pétales retournés du coquelicot. 
canker, idem, IDEM.

cockrose, coprose, Ecosse, Jamieson, Dict., 1879.

fice flout, Northumberland, Britten, Plant-names.

cusk, Warwickshire, IDEM.

calocatanos, gaulois, Marcellus Empiricus ${ }^{1}$ ) (fin du IVe siècle après J.-C.).

[H. G.].

blah na bodagh, cailleagh deary, papin, snannagh, irlandais, J. KEoGI,

1735. [H. G.].

mill, ancien cornique, Williams [H. G.].

llyyad y cythraul (= oil du diable), gallois, Hugh Davies. [H. G.].

pabi coch yr yll (= pavot rouge du blé,) gallois, Richands. [H. G.].

blath nam bodaigh (= the rustic's flower). meilbheag, beilbheag (= a little

pestle), fotlirose (= cornrose), paipean ruadh (= red poppy), crom-

lus (= bent weed), gaélique écossais, Cameron [H. G.].

cathleach-dearg, cochcifoide, irlandais, 0'ReILLY [H. G.].

greac'hel, île de Sein (Finistère), comm. par M. H. LE Carguet.

ros-aer, rosmo'ch goü̈z (= pavots sauvages), breton, GréGoIRe [E. E.].

ro:-moc'l (= roses de cochons), breton, Troude [E. E.].

liolielilio, breton de Tréguier, comm. par M. E. Ernault.

rosylen, breton, D. Le Pelletier, Troude [E. E.]. $\left({ }^{2}\right)$.

ros-aer (= rose de couleurre), Finistère, comm. par feu L. F. SAuvÉ.

liornrose, valmue, volme, liolikeurt (fleur du coq), danois, [H. F. F.].

planiy mak, polný mak, divoliy mak, wlèi mak, puliavec, tchèque,

A. MÜLlER, Wörterb., 1866.

pipaé, slovaque, Mıkosici, Slai. El. im mag. p. 101.

omak, makalj, makounica, poljsli mak, divji makalj, bologlav, bulka, ciganka, crljeni čoek, čambula, fratar, kukoriek, kukurek, kukuriek, kukurik, crljent kuliurik, lala, papuče, pucalica, pucalina, pupulini, turćinak, turčun-cviet, zdur, zdurici, fajdirk, pumpala, prpeluce, prpulice, purpala, purpava, purpelica, pojlski mak, serbo-croate, ŠULEK.

polny mak, maczek żytny, wilczy mak, polonais, LINDE, St. jez. polsk. dzılii mak, polonais de la Prusse, Treichel, Poln. Vulgärnamen.

(1) "Chap. 20, 38 : Fastidium stomachi relevat papaver silvestre, quod gallice calocatanos dicitur, tritum et ex lacte capruno potui datum.

Le nom ganlois semble avoir été fait avec l'onomatopée du cri du coq, quelque chose comme calocata, qui était aussi probablement le nom du coq lui-même. [H. G.].

(2) La correctiou de roz-moc'h en roz-morc'h (= roses assoupissantes), proposée par LE GONIDEC est purement arbitraire. [E. E.].

Le coquelicot est appelé rose de cochon par mépris, par opposition avec la vraie rose. [E.R.]. 
polevoï mak (= pavot champêtre), russe, Schmahlaarsen. [Th. V.]. matchok (= petik mak, petit pavot), petit russien, c. par M. Th. VoLkov. maggons, letton, Stexder, Lett. lex. pipacs, magyar, MıкLosich, Slar. Elem. pipats, vad mäk, magyar, NeviNici, Lexicon. maggunad, esthonien, HUPEL.

lulé kukke, lulé kukje (fleur rouge), albanais, HELDREICH, Nutzpflanzen. hhappapavra (1), ancien arabe d'Espagne, Dozy, Suppl. aux dict. arabes. ben na'man, arabe de Tlemcen et d'Alger, Jourdas, Flore murale. arabe algérien, HaNoTEAU, La Kabylie.

jachailtalnahamem, arabe, SÉraPION L'Axcien, Practica, 1595. na'aman, arabe du Boghar (Algérie) Debeaux, Catal., 1859. khashkhîsh mentsour, arabe, Iвx BEїTHAR Ed. Leclerc), II, 31. abou faraoun, arabe du Haroc, G. Charmes, Une ambassade au Maroc, 1887, p. 65.

hannoun, arabe de la Palestine, Rev. de l'Orient, 1843, p. 419. bugrauna, arabe de Cyrénaïque, Ascherson, (dans RoHLFs, Kufra, 1881). bou-guerioune, arabe de Constantine, CHERBonsede (dans Journ. asiat. 1849, p. 64).

shaegâik, lâle, persan, PoLak, Persien, 1865.

chelingicu ciceghi, turc, Pharmacopea romana, 1869.

guèlindjik tchitchèyi, turc, MaLlouf, Dict. turc.

lale, tatare du Karabach, HoHeNacker, Enumeratio.

dole, arménien, HOHENACKER, Enumeratio plantarum provinciue Talysch. vaïri chashchash (= pavot sauvage), pout, petik, harsnouk (= petite épousée), harsnoudzaghikk (= fleur de l'épousée), harsnalzaghit, arménien, Alishan. [Er. L.].

loschi chassak (les fleurs du coquel.), Turkestan, DragendorfF.

2. Rouge ponceau est une locution qui sert à désigner le rouge de la nuance du coquelicot. - On dit aussi rouge coquelicot.

Comparez l'anglais poppy colour.

Rouge comme un coquelicot.

Loc. franc.

Mas rojo que una amapola.

Loc. espagnole. (1) Dozy dit que ce mot vient du latin papaver. (Il a modifié sen opinion dans un
article postérieur.) 
4. Tout comme jaspe surmonte l'or

Et li lis la fleur de jagliau (I)

Et rose fraiche poonciau.

Cumparaison en ancien français, Zeitschrift f. rom. philologie, 1880, p. 37 ł.

3. Comparer la rose au pavot, c'est comparer des choses qui ne sont pas comparables. Ici parot a le sens de coquelicot.)

Prov. français, Leroux, Dict. com.

4. I a que de cacaracas! Il n'y a que des coquelicots! Se dit d'un terrain aride.

Locut. provençale, Mistral, $T r \cdot d \cdot f e l$.

5. Ne laboure pas au temps chaud

Un terrain sujet aux pavots.

Ain, Statist. de la France.

5. "On dit aux enfans que la fleur du pavot rouge, qu'on nomme lagagne 2 en Languedoc de ce qu'elle fait venir les yeux rouges et chassieux ¿ qui la regarde fort attentivement, s'il ha les yeux tendres et délicats, comme la un enfant), que le manier de ladite fleur les fait pisser au lit. 》 LAURENT JOUBERT, Erreur's popul. 1579, p. 288.

6. Pour empêcher les enfants de courir dans les cliamps de blé, on leur dit, dans la Flandre et le Brabant, que les coquelicots leur suceront le sang; de là le nom flamand de cette plante: bloedzuiger, bloedzuiper (= suceur de sang). Le nom spokebloem signifie : fleur aux revenants. Communication de II. A. DE COCK.

7. En Angleterre les enfants croient que l'action de cueillir le coquelicot fait éclater le tonnerre. Voyez à ce sujet : Notes and Queries, 28 aout et 2 octobre 1880 ; Britte., English plant-names, sub verbo lightnings. - C'est la même croṿance qui a donné lieu aux appellations wallonnes, tonire, fleur (li tonire $\left.{ }^{3}\right)$.

On croit encore en Angleterre que le contact des coquelicots amène des maux d'yeux et d'oreilles. Voy. Britten, Engl. plant-names, sub rerbo blind eyes et sub verbo ear-aches.

(') jagliau = iris, appelé aussi souvent lys des marais.

(2) Cf. le nom arlésien et vauclusien du coquelicot maou d'iu. - Le mot lagagno (= mal d'yeux) sert ordinairement, en languedocien, á désigner diverses espèces de renoncules qui sont également accusées d'être nuisibles aux yeux.

(3) M. 0. Colson me suggère qu'on fait craindre cet accident aux enfants pour les empêcher de pietiner les blés en allant faire des bouquets avec ces fleurs. 
Enfin on accuse cette plante de causer des maux de tête, d'où son nom head-ache. Voyez Britten.

8. Si l'on s'amuse à manier des coquelicots on gagne le mal appelé feu sauvage.

Haute-Bretagne, SÉBILLot, Tradit. II, 33ł.

9. Avant l'éclosion de la fleur du coquelicot, par un plıénomène bizarre, les pétales encore enroulés dans les boutons, se trouvent être, sur le même pied, ou complètement blancs ou complètement rouges. Les enfants jouent entre eux à deviner quelle sera la couleur du bouton cueilli et s'interrogent ainsi : poule ou coq? (1) Celui qui dit : poule, gagne si les pétales sont blancs et celui qui dit : coq, si ils sont rouges.

Euvirons de Paris, rec. persunn.

A Forcalquier le coquelicot est dénommé : 1 Lulipan ou gueringuingaou à peu près indistinctement, lorsque la fleur est complètement épanouic; $2^{\circ}$ gaou-yalin, lorsque les pétales sont encore enfermés dans le calice. Dans ces boutons, les pétales, suivant leur âgre, sont rouges ou roses ou blancs; les enfants en ont fait un jeu, en se demandant, avant de les ouvrir, si c'est un gaou (rouge) ou une galino (rose) ou une galineto (blanc); $-3^{\circ}$ roouro, lorsque la plante est dépourvue de fleurs et qu'on la ramasse pour la donner aux bestiaux.

Comm. de Ni. E. Plauchud.

$$
\begin{aligned}
& \text { ?Fraile, o monja, } \\
& \text { O capuclino que te coja? }
\end{aligned}
$$

Formula de jueguecillo de adivinacion. Coge un niño un capullo de amapola é interroga à otro muchacho. Rota la envoltura, se ve quién haya acertado. Es monja si auı las hojas estan blancas, fraile si se van tiñendo y capuchino si ya estan completamento coloradas.

Espagne, Rodriguez harin, Cantos pop. esp., Sevilla, 1882, I. p. 52 et p. 123.

10. C'est une amusette très répandue, parmi les enfants, de faire éclater avec bruit, sur le front ou sur la main, les feuilles du coquelicot arrangées d'une certaine façon. Beaucoup de noms de cette plante trouvent leur explication dans cet usage.

11. «Les enfants s'appliquent le plateau du fruit du coquelicot ou du pavot sur le front, en pressant hardiment pour en porter l'empreinte. Ils s'amusent quelquefois aussi à écrire arec une encre pourpre qu'ils font sortir des

(1) Dans quelques départements on dit simplement rouge ou blanc, et l'on fait éclater les boutons sur le front. 
pétales du coquelicot en les pressant entre les doigts. \ Flandre, comm. par M. A. DE Cock.

12. “ On fait du coquelicot bien épanoui une charmante petite poupée. Les pétales font l'habillement et les bras, au moyen des fils qui les rattachent. La petite tête fait le visage; le haut de cette tête forme une calotte; les étamines font une jolie fraise ou collerette autour du visage. 》

MADAME DE Genlis, Les jeux champêtres des enfants.

Ajoutons à cette description que les pétales doivent être retournés et ramenés du eôté de la tige et qu'on fait les jambes avec deux brins de graminées. - Dans le livre de Mme de Genlis se trouve une gravure coloriće représentant cette poupée.

«En Ille-et-Vilaine cette poupée est appelée enfant le chœur (la robe des enfants de chœur est rouge).»

Rev. des tradit. pop., 1893, p. 511.

Cette amusette est comnue presque partout. Elle est l'origine de quelques noms du coquelicot, tels que moine, madone, Guillaume, l'anglais poppy. Au lieu de dire faire une poupée on a dit faire un moine, faire une madone, etc.

Comparez le nom chinois du coquelicot : mei jin tsáo (= herba homo formosus), selon J. Hoffirax, Noms des plantes du Japon et de la Chine, Leyde. 1864 .

13. Le coquelicot passe pour détourner la foudre. On en place, dans la charpente, sous les toits, dans ce but.

Pays wallons, comm. par N. 0. Cousos.

\section{DEVINETTES :}

Io tengo 'na cosa

Fatta a rosa,

Rosa non è ;

Bide che è. - Papagno.

Italie méridion., Casetti et Imbriani, Canti pop. d. prov. mer., t. I, 1871, p. 82.

Con mi cara encarnada

I mi ojo negro

Y mi vestido verde

El campo alegro. - La amapola.

Espague, Piodriguez Marin, Cantos pop., 1882.

Redondiinho, redondiinho,

Munto vermelhiinho,

E com munto pentilhiinho. - A papoula.

Province d'Alemtejo (Portugal), Archivio delle trad. pop., III, 119. 
Vermelhinha, vermelhinha

No meio pelludinha. $-A$ papoila.

Bragance (Portugal), Revista lusitana, t. III. p. 90.

14. On se sert des fleurs de coquelicot pour joncher le sol le jour de la Fète-Dieu.

Divers départements de la France.

15. Les habitants de la lune ont des chauves-souris en guise de vaches et des coquelicots en guise de blés; s'il s'en trouve sur la terre c'est que ce sont des échappés de la lune.

Danemark, Kristensen, Folkeminder, IV, 366, 209. [H. F. F.].

16. Les enfants dans les champs mangent les graines mùres du coquelicot avec plaisir : ce qui leur amène quelquefois un sommeil subit dont les parents ont peine à se rendre compte.

"En Roumanie on croit qu'il suffit de coucher près d'un coquelicot, pour s'endormir. 》

Comm. de M. A. Gorover.

\section{PAPAIER SOMNIFERUM LINNÉ). - LE PAVOT.}

$\mu \dot{r} \% \omega \nu$, grec ancien.

1. - Noys :

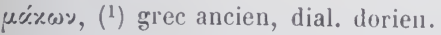

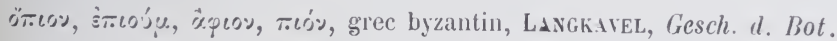

тò ù.̧⿻心㇒, grec moderne, Heldreich, Nutzpfl.

бuб'se, grec mod., Germano, Гoc., 1622.

papaver, mecon, latin.

miconum, latin du Moyen-Ige, Germania, 1888, p. 307.

papaver album, pupaver sativum, papaver hortense, anc. nomenclature. pavot noir, m., anc. fr., C'onseils contre la famine, 1546.

pavot, m., pavot blanc, m., pavot noir, ( $\left.{ }^{2}\right)$ m., pavot des jardins, m., pavot cultivé, m., français.

pavot privé, m., anc. fr., Livocier, Hist. des plantes, 1584.

pabot, (prononcez pabó) m., Poitou. - Libourne (Gironde), c. par

(1) Pour l'étymologie du grec uxixwy et du latin mecon, voyez: Archiv $f$. lateinische Lexicographie, 185', p. 118-119.

(2) Le pavot est tantôt appelé pavot noir et tantôt pavot blanc, selon que les variétés cultivées ont des semences blanches ou noires. 
M. L. Durand-DÉgrange. - Genouillac (Charente), Rev. des pat. gallorom., III, 194.

pabót, m. (prononcez pabott), Lot. - Tarn-et-Gar. - Haute-Garonne. Hautes-Pyrénées.

pavoit, m., Luchon (Pyrénées), J. SACAzE. - Forcalquier, comm. par M. E. Plauchud.

pobouót, m., languedocien, comm. par M. H. FAU.

peró, m., Issoire (Puy-de-Dòme), c. par feu J. BAREIRE.

peutäï, m., Bourberain (Côte-d'Or), E. Rabiet, Pat. de Bourberain, p. 17. pabou de jardin, m., Charente-Inf., comm. par M. E. Levarié.

papaver, m., anc. provenc., RAYNoUARD, Lex. rom.

paver, m., anc. prov., Raynouard, L. rom. - Gard, comm. par M. P.

FESQLeT.

pavier's, m., franç. du XIVe s., Escallier, Rem. s. le pat., suivies d'un vocab. du $X I V^{\circ}$ s., 1856, p. 476.

paveux, m., dans le Corbonnais (Normandie), Joret.

pavaur, m., pavoir, m., pawè, m., wallon, GRANdGAGNage.

lob paioir, m. (= double pavot, variété cultivée pour l'agrément), liégeois,

Forir, Dict. liégeois.

pavot double, m., (la variété cultivée à pétales multiples), pavot de

Hollande (= idem), français, Fillassier, 1791.

pavois, m., fleur de pavois, f., anc. franç., Lacurne de SAiNte-Palaye,

Dict. (Edit. Favre).

paparri, m., Var, AMic, Considérat., 1837. - Bouches-du-Rhône, Ville-

Neuve, Statist.

pavon, m., Bas Valais, Gilliéron, Pat. de Vionnaz.

ponciau franc, m., Loiret et Eure-et-Loir, c. par M. J. PoQLET.

pabou, m., Poitou, Rev. de philol. franc., 1893, p. 116.

endormie, f., anc. franc., J. Victor, Tesoro, 1609.

cascall, m., Pyrénées orient., Comparyo, Hist. nat.

yèp à l'ol, wallon, Feller.

olivette, (') f., anc. franc., Duchesxe, De stirpibus, 1544; Philitre, Tresor

des remedes, 1555. - Environs d'Amboise, comm. par M. E. Lelièrre. oliette, f., anc. fr., Vocabularius quattuor linguarum, 1516. - Picardie.

- Flandre française - Berry. - wallon.

olivotte, f., Meuse, Labourasse, Glossaìe. - Haute-Marne, comm. par M. l'abbé M.ırchal.

(1) Le pavot est ainsi appelé parce qu'il remplace l'olive pour faire de l'huile. Cette huile est appelée en français huile d'xillette. 
aillette, f., français.

ullieto, f., Corrèze, Béronie, Dict. du pat. du Bus-Limousin.

ouyette, f., Mons, Sigart, Gloss. montois.

euyette, f., Saint-Pol (P. de C.), comm. par M. Ed. Ednoxt.

chanotte, f., Meuse, Labourasse, Gloss.

claquiróto, f., Ouest du département de Lot-et-Garonne, comm. par

M. l'abbé L. DARDY.

papavero, italien.

papaveru, sicilien, Biaxci, Flor'a d'Avola.

papaver bianc, piém., Coll.1.

papàer, stormia, Brescia, ZERsı.

papêvar, romagnol, MoRri.

papàveo, Gênes, Casaccia, Diz. genou'.

papatêr da ort, Plaisance, Bricciforti, flora piac.

paparina, sicilien, Cupaxi, Hortus callolicus, 1696 ; Mortillaro.

paie, Gardena (Tyrol italien), Visather.

papola, italien, Duez, Diz. ital. fr.., 1678.

poupoulón, (pavot slouble cultivé), Pavie, Manfredi.

papayno, napolitain, Puotı.

pupla, Ferrare, Ndwini, Vocab. ferrarese, 1705.

pabauiti. Sardaigne du Sud, Monts, Fl. sard.

pappardo, anc. ital., Casaccia, Dio. yen.

pappardolo, ital., Casaccia, Idem.

papimbre, m, papimbele, 111., paprimberu, in., Mbruzzes, Fixiмone. cucuzzeddi di sounu, siciiien, Lagusı, Erbuario, 17亿.

beltis, m., sarde méridional, SPAxo.

dormidera, espagnol.

adormidera, espagnol, J. VıстоR, Tesoro, 1609. - Baléares, Mırès, Catal. cascall, catalan, Povio, Thesaurus puerilis, 1580. - catal. moderne.

cascay, Baléares, Marès, Catal., 1880.

adormideira, galicien, VALLADARES.

dormideira, portugais.

mac, roumain.

mac alb, mak de gredine, roumain de Transylvanic, Fus\%, Trivialnamen. migo, auc. haut allemand.

mûhen, mage, mân, môn, moyen haut all.

molin, allemand.

weiss muech, guerte muech, allem. de Transylvanic, Fusz, Trvialn.

mô, m., Silésie, document de 1568, Frominan, Die deutsclie Mund., IV, 1857, p. 177.

moh, silésien, WeInuold, Sclules. Wöıt., 1855. 
magen, Tyrol, Carinthie, Salzbourg.

mayeel, m., canton des Grisons, StALDER.

maon, Altmark, DANxeIL.

ölmagen, m., magen, Souabe, ScHнid.

mân, Unterwesen, Focke, Pflanzenn., 1870. - Goltingue, Sснамвасп.

stinlibüsch, stinlibluomä, satlelbock, canton de Saint-Gall, Wartмıмx. makuwken, plur., Prusse, Frischbier, Preuss. Wört.

machoea, papaver, Grisons, Engadine, Durheis, Schw. Idiot.

mageel, Grisons, IDEM.

liolben, m., Lucerne et Berne, STALDER. (Der rundlichen Gestalt wegen). magsaamen, canton de Berne, IDEM.

pawô, Luxembourg, J. WEBER.

tamm heul, anc. flam., Dodonaeus. [A. DE C.]

heul, heul:aad, heulbloem, maanliop, slaapbol, slaapkruid, hollandais et flamand. [A. DE C.]

zwarte heul, slaap-bol, flamand, DE GORTER.

honsdrooze, meeliop, oliette (mot d'origine française), slaapbotte, poeierburstel, flamand, DE Bo, Idiot. [A. DE C.]

balewurt, anglo-saxon, Cockaxie, Leechdoms, 1866.

garden poppie, angl., Minsueu, Dict., 1623.

chessbolls, cheesebouls, anglais, CotgRave, Fr. dict., 1650.

popp!y, anglais, Cotgrave, Idem.

codalian, collaidin ban, gaélique écossais, Cameron. [H. G.]

calladın poipin, irlandais, J. KEOGH, 1735. [H. G.]

llysiau 'r cu'sy (= herbes du sommeil). pabi dof (pavot domestique; pabi

est l'angl. poppy), gallois, J. DAvies, 1632. [H. G.]

pabi lledfergin (= pavot domestiqué), bwhwg lledfegin, gallois, Meddygon

Myddfai. [H. G.].

llys y cusy (= plante du sommeil), drewy, drewlys (= la plante

puante), pabi, bwlwg ffrengig (= bwlwg français), gallois, HugH DAvies.

[H. G.].

ros-qy (= roses de chien), breton, GRÉG0IRE. [E. E.].

ross-qui, breton de Vannes, L'Armerie. [E. E.].

ros-moc'h (= roses de cochon), pauot, breton, Nomenclator. [E. E.].

roz-moc'l, breton, TRoude. [E. E.].

beli mak, pitomi mak, veliki mak, pitomi makalj, bili pitomi liukurik,

serbo-croate, Šulek, Jugosl. im. bil.

mak, polonais, bosniaque, slavonien, Linde, St. jez. polsk.

máli, tchèque.

patrak, polonais de la Prusse, Treichel, Poln. Vulgärn.

mak samossiy, petit russien, c. par M. Th. Volkov. 
mak, russe, serbe, bulgare.

hašis, bulgare.

hašas̆, albanais.

magona, lithuanien, JАсову, Litauische Pflanzenn., 1884.

mák, magyar.

makmay, magyar, Linde, St. jez. polsk.

evö mak, magyar, Fusz, Tivivialnamen.

málio, tsigane, Mrḱosici.

khashkhash, arabe, turc.

abou 'l noum (= le père du sommeil), arabe.

xahxiech, Malte, Delicata, Flora melil.

alfenith, arabe, SÉrapion l'Ancien, Practica.

djouldjoulan el hhabecha (le pavot noir), arabe, Leclenc, Révélatious, 1874.

acu hashassu, turc, Phaimacopea romina, 1862.

chashchash, arménien, Alishan. [Er. L.].

makou, Turkestan, G. Capus, Plantes cultirées, 1881.

afiun guli, laili qazaq, Turkestan oriental, Sнıw, Vocabul.

afioun, arabe, hindonstani.

botink, kurde, Garzoni, Grammatica kurda.

post, hindoustani.

tschacht-tschach, tatare, Gueldenstaedt, Reisen, 1787, I, 188.

cur, langue inconnue du pays de Wiesbade au XII s., Descemet.

2. On cultive pour la graine deux varietés de pavuts bien distinctes l'une de l'antre, quoiqu'elles appartiennent toutes les deux à la mème espèce : le papaver somniferum. En Europe, on en cultive une forme vigoureuse, généralement bien ramifiée, à tiges hautes et raides et à fleur's violettes; c'est celle que l'on nomme habituellement æillette. On en distingue trois sousvariétés : l'œillelte grise ef l'xillette bleue, ainsi nommées de la couleur de leur's graines, et l'oeillette aveugle, dont la capsule reste complètement close à la maturité, ce qui met le cultivateur à l'abri de toute perte de graines au moment de la récolte et pendant les transports.

Vilmorin, Exposition de 1878; les produits agricoles non alimentaires, p. 85.

\section{LA TÊTE DU PAVOT EST APPELÉE :}

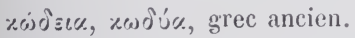

codion, cadion, latin du Moyen- $\mathrm{g}$, Diefenbach.

papaveris capitellus, latin du Vo siècle, Cassius, De medicina. Edit. de Leipsig, 1879.

cap de pabot, languedocien. 
tête de pavol, f., français.

tièsse d'ouillette, f., Morlauwelz (Pays wallon), Semertier.

pot, m., Saint-Pol (Pas-de-Calais), c. par M. Ed. ЕDнохт.

senolte, f., Toul, Domgermain, L. Avau.

tchenatte, f., Plainfaing (Vosges), rec. pers.

magueja, (avec $j$ français), Gardena (Tyrol italien), Vinather, Gröllen. mâciulie, roumain, c. par M. A. Gonover.

magenliopf, f., anc. h. all., Germania, 1888, p. 302.

mohnhaupt, magsamenschlauch, magsamenhäuptlein, allemand, DUEz, 1664.

kolpe, Carinthic, LEXER, Kärnt. Würt.

meetiop, flamand, DE Bo, Westfl. Idiot. [A. de C.].

makouice, f., tchèque, Palkowitsch.

makowica, polonais, LINDE.

rommânet el khashkhash, (c. à d. grenade du pilvot), arabe algérien,

LECLERc, Trad. d'Ibn Bë̈thar.

kik nar, liuk nar (= grenade jaune , Turkestan. - Perse.

4. - Le suc épaissi du papaver somniferum employé e:! médecine et comme soporifique est appelé :

ӧтьо, grec ancien.

opium, latin.

papareris lacrima, sopora, latin de la fin du ve siècle, Marcellus

Fupiricus, cité par Meier, Gesch. d. liot.

oppium, opion, latin du Moyen-Age.

opium, m., français.

endormie, f., anc. fr., Duez, 1678.

doirmant, m., wallon, Semertier.

oppio, alloppio, italien.

silormia, Brescia, Melchori.

indormia, Padoue, Patriarchi.

opi, Mantoue, Cilerubini.

anficio, portugais.

magenmillich, magensaft, ancien allemand, DiEFENBACH.

nicklasruh, nickelsruh, Prusse, Frischbier, Preuss. Wört.

schwartznansat, anc. 1. all., Germania, 1888, p. 307.

appion, arménien, Alish.A. [Er. L.].

afioun, arabe, ture.

afioun roumi (= opium thebaïcum), arabe égyptien, Alpisus, De medic.

xeypt., 1645, fo 134, verso.

tärjal, persan, Stolze und ANdreas. 
tì'yâk, Afghanistan, Dors.

a-fou-yony, chinois ( ${ }^{1}$ ) (d'après un auteur de 1552), A. DE Candolle, Origine, p. 321.

Voir pour l'historique de l'0pium une longue bibliographie dans MÉrat, Dict. de matière mérlicale, 1833, t. V, p. 64 et suiv.

A. H. L. HeER, Versuche die früheren Spuren einiger Handelszweige des Alterthums zu erklären (Dans Abhandl. d. hist. phil. Classe d. k. Gesellsch. d. Wissensch. zul Göttingen. Erst. Banl. 1831), p. 7-10. - W. Hert7, Sage vom Giftmädchen daus Abhandl. d. k. bayer. Akad. d. Wiss. KI. I. XX. Bd. 1), p. 72-73. - KempFer, Amonitates exotica, 1712 p. 642-645.

Voir surtout l'important article consacré à ce produit par Flückiger and Hanburi, Pharmacograplia, London, 1874, p. 40-60.

On peut consulter aussi A. DE CANDoLle, Origine des plantes cultivées, 1883, p. 319 et suiv.

Sur les moyens employés par les anciens pour obtenir le suc du pavot, voyez Daremberg, Eurres d'Oribase, 1, 641.

Sur la préparation pharmaceutique appelée au Moyen-Ige diacodion Galeni, diacodion, on tronvera d'utiles renseignements dans N. Prepositus, Antidotarium majus, 1536. in-fol., fo 29 a et Artidotarium mimus, 1562, in-fol., fo 378 b.; P. SUARd, Thesaurus aromatariorum, 1526, in-fol., fo $6 \mathrm{~b}$.; DE Bosco, Luminare majus. 1536, in-fol., fo $12 b$.

AleX. de Tralles, médecin grec du VIe s. apr. J.-C., lont l'œuvre a été traduite en latin à peu près à la même époque, parle d'une préraration appelée oppomiconum que le commentateur de son ourrage (XIII siécle) explique par succus papaveris.

5. « Che ti possa morir come i papaveri co le gambe distese e il collo torto. 》

Malédiction facétieuse, Generici per la maschera d'Arlecchino.

Milano, in-12, s. d. (vers 1860 ?, p. 23.

6. «Drizzare i papaveri ne’ gambi. »- Redresser les pavots par la tige, c.-à.d. rompre ou ruiner une affaire.

Italien, DuEz, Diz. ital., 1678.

7. Lorsque dans une sociétí il se fait tout à coup un profond silence, par suite du manque de conversation, quelqu'un dit : dobze mak siać (= bon temps pour semer les pavots!). - On sait que la graine de pavot est très fine et susceptible d'ètre emportée par le vent.

Polonais de la Prusse, Treichel, Volksth. $\$ \mathrm{X}$.

(1) L'opium ne semble las connu, en Chine, avant le XIZI ${ }^{c}$ siècle. A. DE GandolLE, Origine, p. 321 . 


\section{FOLKLORE}

Voir : Michael Friedericus Locinerus, Papaver ex omni antiquitate erutum, nummis, gemmis, statuis et marmoribus incisis illustratum, Noribergix, 1719, in-4. 198 p. [Se trouve à la Bibliotlèque Nationale sous la cote $\mathrm{J}$ 1200, 3.]

Voy. au point de vue mythologique, Dienвach, Flora m!th., p. 117 et suiv.

\section{DEVINETTES.}

Ein kleines Haüschen ist ganz mit einem Groschen bedeckt. - Ein Mohnkopf. Prusse, FriscuBier, Preussische Pflanzen Ractsel (1).

Ein lileines Speicherchen, ganz gedeckt mit einem Groschlein. Was ist das? - Ein Mohnhopf.

Devinette lithuanienne, ScHLElcuEn, Litauische Maerchen, etc., 1857.

Rat amal was ist das: a Stingele, a Panzele und obendrauf a Kranzele. Mayn. Carinthie, ZWanziger, Veræeichn., 1888.

Es ist ein Pommeranzel, Hat ein schönes Kranzel Und ein langen Stiel. - Mohn.

Tyrol, Zeitsch. d. Ver. f. Volksk., 1895, p. 15 '.

Ribele, rabele, Z' unterst a Stabele, Iitten a Panzele, Z' oberst a Kranzele. - Mohn.

Tyrol, Zeitsch. d. Ver.f. Vollisk, 1895, p. 154.

Stoji " poli hiulka,

Na té hiolky kulka, A $v$ té kulce

Na tisice.

(Im Feld steht eine Stange, auf der Stange eine Kugel und in dieser kugel wohl bei tansend andre. - Mohn).

Moravie, Zeitzch.f. d. d. Myth., IV, 372.

(1) Dans la même collection $\left(\mathrm{n}^{\circ} 55\right)$ se trouve une autre devinette très longue et très compliquée relative au parot. - RоснноLz, schweiz. Volksracths. ans der Aargau, n 40, donne une devinette relative à celte plante. 


$$
\text { P.PAVER ARGEMONE. L. }
$$

papaver spinosum, argemone, anc. nomenclature.

argémone, f., pavot épineux, m., français, LIGER, Le jardinier fleuriste, 1718 , t. I, p. 99.

maou d'uey, Var, Havri, Catal. du Var.

esparpai, environs d'Avignon, PALus, Ciatal.

ander, arrondissement de Brive (Corrèze), G. DE LÉPINAY.

paparinicchia, sicilien, CUPani, Hortus, 1696.

paparinedilu, sicilien, LAGusi, Erbuario, 1742.

madonine smorte, Brescia, ZERSI.

battürus (= blouse rouge), surele, piémont., Colla.

mak polevy, (= pavot champêtre), petit russien, c. par M. Тн. VoLKov.

okolik, okoliclia, serbo-croate, ŠulEk.

tshashmak, tshashmil, tshèshmak, arménien, Aushax. [Er. L.].

arghamoûni, arabe syrien, BERGGREN, Guille.

dikenlu kliachlihach, turc, BARBIER DE MEINARD.

\section{I'APA TER IYBRIDUM. (LINNÉ.)}

pabaùli, Sardaigne du sud, Horis, flora.

papaveru, pupuza, Sardaigne du nord, Moris.

paparina masculina, île d'Ústica, CALCAra.

paparinedda spinusa, Sicile, Bianca, Flora.

papoila peluda, portugais, Brotero, Flora.

peprin, Malte, Delicata, Flora mel.

bou-gara'ouin, arabe tunisien, PRAx.

\section{'PA'A TER SETIGERUM. (DE GANDOLLE).}

papari, Var, Hanre, Catal. du Var.

cascay borl, Baléares, MARĖs, Catal.

$$
\text { PAPA VER IULBIUM. (LINNÉ). }
$$

madonine, Brescia, ZERsi.

papoila longa, portugais, Brotero, Flor'a.

\section{GLAUGIUM LUTEUM. (ScopoLI). LE PAVOT CORNU.}

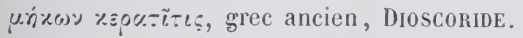

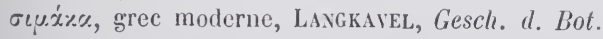

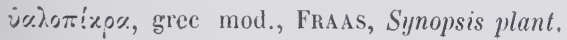




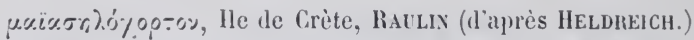
glauceum, latin du $\mathrm{I}^{\mathrm{er}}$ s. ap. J.-C., Colduelde, X, 104. glaucion, menithe, latin, PLine.

papaver cornutum, paparer ceratites, anc. nomenclat, Duchesse, De stirpibus, 1544.

paparer corniculatum, anc. nomencl., Fuchsies, 15i6. (Corniculatum a fructu (juem producit, cornu modo inflexn, nominatum est.)

celidonia agrestis, memita, memithe, ylaucium. glaucus agrestis, nomenel. du moyen-age, Mowat, Alphita.

menuta, ane. nomencl., J. Caves, Liv're d'heures.

chelidonium glaucium, nomencl, de LișÉ.

glaucium flaum, nomencl. de Cravtz.

parot cornu, m., anc. fr., Ducuesxe, De stirp., 1514. - franç. moderne. grande esclere, f., anc. fr., J. Cants, Livre d'heures.

cliélidoine ylauque, f., français savant.

cascall cornut, m., Pyrénées-0rient., Companyo.

bé de corlu, m., Bessin et Cotentin, Joret.

corblet, m., littoral de la Manche depuis Dunkerque jusqu'au Harre, Société philomathique de Paris, 1860, p. 33.

dindoulièro grosso, f. Var, HAxrs, Catal.

dindoulièro, ruélo jaouno, f. provenç. mod., Lıoss, Véyét. utiles des Bouches-du-Rhòne.

erbo doou faioou, f., envir. d'Avignon, Palux, Catal.

erbo de la peiriero, f. aoureieto, f. Ipt, Colignos, Flore.

erbo dei peiriero, f., erbo dei dindoureto, f., erbo de la roucassio, f.,

Forcalquier, comm. par II. E. Plauchud.

erbo doou favioou, f., erbe dé nière, f. Mrles, Laugier de Chartrouse, Nomenclat.

glaucio, paparero cornuto, paparero marino, cienerognola, italien,

TARgioxi, Diz. bot.

papaveru cornutu, Sard. du Nord, Monis, Flora.

pabaùli cornutu, Sard. du Sud, IDEM.

paparina cornuta, sicilien, LAGLsI, Erb., 1742.

caulu marinu, sicilien, CuPANi, Hortus, 1696.

cascall cornut, catalan, Costa, Flora de Cat.

cascay bord, guixo bord, Baléares, MARĖs, Cat.

cascall mari, catalan, LACAVAlLERIA, Gazophylacium, 1696.

dormidera marina, espagnol, PALMirexo, locab. del human., 1575.

mapola mariña, galicien, Cuveiro, Dicc. gall.

lechuga salvage, îles Canaries, WEBB, Ilist. nat. des Canaries.

llwyllas (= bleu grisâtre), gallois, Hugh Davies. [H. G.]. 
louzaouenn ann darvoed (= herbe aux dartres), breton du Finistère, comm. par feu L.-F. SAUvé.

rostopaść, polonais, Šulek, Jug. im. Bilj.

morski mak, polemorsli mak, polemorni mak, rogati mak, rogati matialj, morska makorina, kostenjaca, ceduljka, serbo-croate, ŠULEK.

szarvas màk, magyar, KolBANI.

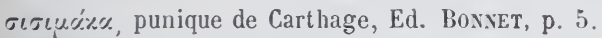

mamita, arabe de Tunisie, Ed. Bonnet.

dzoiou chashichash (= pavot de la mer), arménien, Alishas. [Er. L.].

mamîta, scheqiq el karn, arabe syrien, Berggrex, Guide.

\section{ע. - USAGES :}

Les Romains en faisaient un collyre pour les yeux sous le nom de diaglaucium. Vovez: Bull. ar`chéol. du Comité, 1887, p. 398.

«En exprimant sur les dlartres le suc âcre et jaune dont les tiges de la Chélidoine glauque (louzaouenn ann-(larioed) sont remplies, on parvient à s'en rlébarrasser rapilement. On emploie également ce caustique contre les verrues. »

Finistère, comm. par M. L.-F. SAUVÉ.

\section{GLAUCIUII CORNICLLATUM. (GURTIS.)}

celidonius masculus, nomenclature du xi॰ s., Cavus, L'Opera salernitana, p. 52.

clielidonium cormiculatum, nomenclat. de Lisxé.

glaucium phœnicium, nomencl. de GaERTXer.

erbo de peirieiro, f, env. d'Arignon, Palts, Catal.

ruélo banarudo, f., provens. mod., RÉGuis.

amapola boyuna, espagnol, Fragoso, Cururgia univers., 1621.

cennerugio, italien du xie siècle, Cawus, L'Opera salern., p. 52.

papauna, paparóana, roumain, Ciнac, Dict. daco-rom.

\section{MECONOPSIS GAMBRIGA. (VIGUIER).}

papaver cambricuni, nomenclat. de Lixite.

pavot jaune, pavot du pays de Galles, français, Fullassier, 1791.

\section{R(EMERIA HYBRIUA. (DE Gaxdolle).}

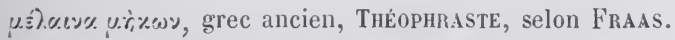

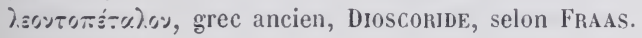


ababol moralo, amapola morada, roseta morada, espagnol, Colmeino, Dicc. de los nombres.

rigl el ghorâb (id est pes corvinus), arabe égyptien, DELILe, Flora æxypt., 1824 .

\section{ARGEMONE MEXICIANA. (LINNÉ).}

carduus flavus, nomenclat. des officines, Rosenthar.

argemone du Merique, f., pavot cormu du Mexique, m., figue d'enfer, , figue du diable, f., pavot épineux. m., chardon béni dles Antilles, m., français, Fillassier, 1791.

charlon, m. Ile de France (Afrique), BORI DE SAlnt=Vincent, Voyage dans les îles d'Afrique, 1804, t. I, 165.

chaidon béni, m., La Martinique, MoReaU dE Jovnès, Iyyiène milit. des

Antilles, 1816, p. 83.

adormidera espinosa, espagnol.

thistle, anglais des Intilles, WEst, 1794.

yellow thistle, anglais colonial, Grise BACH.

prickly argemone, anglais.

\section{CIIELII)ONIUM IIJUS'. (LINNÉ). - LA GRANDE ÉGLAIRE(').}

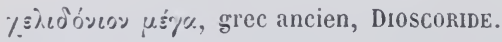

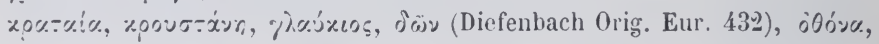

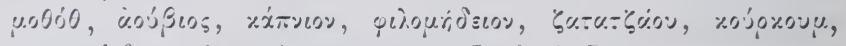
usour,

chelidonium, latin, PLjNe.

hirmulinina, latin du vio s., Apuleus, De medicam.

celidonia, celidonia major, herba petiginaria, herba glaucia, nomenclat.

du moyen àge, Mowat.

herba hibundinum, nomencl. du moyen âge, Continens Rhasis, 1486.

chelidonia, anc. nomencl., Descenet.

hirundina, nomenclat. du moṿen àge, Bibl. de l'éc. des ch., 1869, p. 332. herba hirundinata, erundina, erundinia, latin du moyen âge, Diefenbach. arundinina, herba arundinea, nomencl. du xve s., Cames, L'Opera salern., p. 52.

celedonia, lat. du xie s, Mém. de la Soc. de Liny., VI, 364.

celidantia, anc. nomencl., Zeitsch. f. d. Alterth., 1872, p. 362.

(I) Le nom de petite éclairc est consacré au Ranuxculus ficaria, L. 
chelidonium vulyare, chelidonium majus, hirundinaria major, anc. nomenclature.

esclairø, f., esclère, f., esclayre, f., ancien français.

eclaire, f., grande éclaire, f., français moderne.

estlère, f., anc. franç., L'Arbolayre, 1490.

echclairo, f., Brive Corrèze, G. DE LEPINAY, Noms.

esclairo, f., sud-ouest du Languedoc, Duboul.

erbo d'esclayre, f., Castres, Couzinié, Dict. pat. fr.

herbe à l'éclaire, f., Anjou, Desvaux, Flor'e de l'Anjou.

herbe à la claire, f., Centre, Jaubert, Gloss.

herbe d'écllaire, f., herbe de l'écllaire, f., Deux-Sèvres, comm. par

M. B. Sочché.

erba de la clera, f., Velay, Deribien de Cheissac, Vocab. du pat. du

Velay.

herbe du claire, f., env. d'Etale (Luxembourg belge), rec. pers.

dchotte de kiere, f., (dchotte = herbe), Ban de la Roche, H.-(r. OrEnLin. cler, m., clere, f., anc. fr., Lacunne de SAinte-Palare, Dict.(Edit. Favre'.

claire, f., Langres, Mulson, Vocab. lanyrois. - Eure, RoBis, Dict. -

Norm., JoRET.

grando clairo, f., environs de Toulon, Patout, Plantes médicin.

yrande claire, f., Haute-Marne, c. par M. A. Daguiv.

clariana, f., Arrens (Hautes-Pyrénées), c. par M. M. CAMÉLAT.

clarégea, f., Luchon (Pyrénées), J. SACaze.

tiaire, f., étiaire, f., Vosges, Haildant.

rêchaire (1), Montbéliard, Contejean.

rêquièrio, f., Árbois (Jura), conmm. par M. F. L. DornoN.

clareto, f., Haute-Garomne, Tourxos. - Montauban, Gaterau. - Var,

Hayry. - Avignon, Palux. - Bouches-du-Rh., Villexelve. - Ville-

franche (Haute-Garonne) et Aude, c. par .I. P. FAGot.

clario, f., esclaïro, f., Argelès (Hautes-Pyrénées', comm. par II. P. TARissax. erbo de santo-Claro, Gard, c. par M. P. FEsquet. - Var, Hanry. -

Avignon, Palun. - Bouches-du-Rh., Villeneuve. - Langued., c. par

M. A. JEAnRos:

chelidoine grande, f., anc. franç., J. BEGuin, Elemens de chymie, 1665,

p. 368 .

chélidoine, f., grande chélidoine, f., français savant.

cherungne, f., fr. du xï s., Bibl. de l'Éc. des Chartes, 1869, p. 332.

cheloingne, f., anc. fr., J. Cisuss, Récept., p. 6.

(1) C'est le mot éclaire préfixé d'un $r$. (CONTEJEAN). 
celıdonia, f., anc. provenc., Raynouard, Lex. $r$.

celydoine, celidoine, f., anc. franç., L'Arbolayre, 1490; Соввісном, 1525; REINSCH.

salidoine, f., wallon du XVo siècle, J. CAmus, Manusc. nam.

celidone, f., anc. frans., GACHEт, Manuscrit d'un glossaire du $X V^{\circ} s$.,

1846; Conbichon, Le propr. d, ch., 1525; Reinsch (dans Arch. de

Herrig, 1880, p. 174).

cellande, f., anc. franç., GoDEFrold, Dict.

celande, f., chierlande, f., wallon du XV' siècle, J. CAyus, Un manuscrit namurois.

celoingne, f., wallon du XVe siècle, J. Cayus, Manusc. nam.

celonyne, f., anc. fr.. J. Cayus, Récept, p. 7 et 14.

soloyne, f., Verviers, Lejeune, Flore des env. de Spa,. - Spa, LezAACK,

Flore. - Rochefort (Belgique), Bull. de la Soc. de bot. de Belg.,

$1883,2 \mathrm{e}$ p., p. 14.

solayne, f., wallon, Grangignage, Dict. wall.

selogne, f., Chambéry, Colla.

sêlogne, f., sûlogne, f., saleügne, f., syerlogne, f., cherlogne, f., tcher-

logne, f., surlogne, f., sôdrogne, f., sandrogne, f., yèp du bo, f.,

(= herbe du bouc, à cause de son odeur), wallon, Feller.

grantt sologne, f., liégeois, Forir, Dict. liég.

sarlongne, f., anc. fr., J. Cauus, Récept., p. 14.

seyogna, f., segognarda, f., felogne, f., Suisse romande, Vicat.

cigognar, m., fegogna, f., fegognarda, f., Suisse rom., Bridel, Gloss.

felonyne, f., anc. franç., A. Pineus, Hist. plant., 1561; Du Piner, 1660.

felougne, f., franc. mod. selon quelques botanistes.

felougno, f., Aveyron, Vayssier.

soulougno, f., dauphinois, Mistral.

feloujo, f., prov. mod., Mistral.

férougeo, f., Yar, Амic, Consid. sur Briynolles.

erbo feroujo, f., (= herbe farouche, herbe féroce, ainsi appelée à cause

de sa grande âcreté), erbo dei berbi, f., Forcalquier (Basses-Alpes),

c. par M. E. PLauchud.

saradougno, f., Gard, c. par M. P. FESRUET.

seruejo, f., provenc. mod., RÉGuIs.

saraluegno, f., sarajhueyno, f., languedocien, Sauvages, Dict. lang.

sarigónio, f., salaránio, f., arr. de Saint-Pons (Hérault), BARTHÈs, Gloss., 1873.

erba saloni, f., Pyrénées-0rient., Companyo, Hist. nat.

arondeliere, f., anc. fr., F. P., Indiculus universalis, Lyon, 1667, p. 49.

arondelerie, f., anc. fr., RexÉ FrançoIs, Essay des merv. de la nature

1622, p. 91. 
herbe aux airondelles, f., anc. fr., VictoR, Tesoro, 1609.

herbe aux hirondelles, f., herbe à l'hirondelle, f., français.

ierpe d'arondiéle, f., Valenciennes, Hécart, Dict. rouchi.

dindouliero, f., Var, Hanky ; Amic. - Forcalquier, c. par M. E. Plauchud. aoureïeto dindouliero, f., Apt, Colignon, Flore.

dindouniero, f., erbo à l'irondo, f., erbo à la dindouleto, f., erbo de San Jan, provenç. mod., RÉGuis.

herbe aux verrues ( $\left.{ }^{1}\right)$, f., Haute-Marne. - Doubs. - Normandie. -Orléanais.

herbe aux poireaux, f., Haute-Marne.

arbe aux pouraux, f., Haute-Marne, comm. par M. l'abbé ManchaL.

herbe aux porions, Normandie, Jonet.

erba de las boruyas, f., l'yrénées-0rient., Conpanyo.

erbo de los borrugos, f., Aveyron, Vayssier, Dict.

erbo dei barrugo, f., Forcalquier, comm. par M. E. PLauchud.

erbo de las bar'rugos, f., Lauraguais (Haute-Garonne), c. par M. P. Fagot. erba deis mouridzas ( = herbe des verrues), Brioude (Haute-Loire), c. par

M. P. LE BLANc.

erbo de las varudzas, f., Corrèze, G. ve LéPINar, Noms.

erba verruga, f., Nice, Risso, Ilist. nat.

erbè de la vardza, f., erbè de lè maillè, f., Issoire (Puy-de-Dôme), c. par

M. J. BABEIRE.

bourudaïro, f., (= verrucaire), Le Buisson (Dordogne), rec. personn.

erba de la jaunisse, f., Lyonnais, Puitspelu, Dict.

dchotte de jünisse, f., (dchotte = herbe), Ban de la Roche, ObenLin.

lierbe dè jaunisse, f., Vosges, Hallant, Flore.

dzônisso, f., environs de Limoges, rec. pers.

jaounisso, f., Est du Lot-et-Garomne, c. par M. l'abbé L. DARDY.

jaounisse, f., Libourne (Gironde), comm. par M. DuRAND-DÉGrange.

erbo de billo, f. Lauraguais (Haute-Garonne), comm. par M. P. Fagot.

(On fait avec la plante une tisane pour combattre les épanchements de bile.)

herbe à la vue, f., Rouvray-Saint-Denis (Eure-et-Loir), c. par M. J. P0QUET. erba de l'éstérisiegna, f., canton du Vigan (Gard), Rouger, Topographie. erbo de la blonqueto, f., blonqueto, f., Aveyron, Varssier, Voc., erbo de lo blonqueto, f., Laroquebrou (Cantal), c. par M. J. MaLvezin. erbo del vent, Brive (Corrèze), G. DE LÉPINAY, Noms.

(1) Le suc jaune et caustique de la tige de la plante, applique sur les verrues, les fait disparaitre assez rapidement. 
herbe au diable, f, Côte-d'Or, RoYer, Flor'e.

chadiou, m., Poncin (Ain), rec. pers.

yape, f., jagouasse, f., Centre de la France, Boreau, Flore.

herbe au lait jaune, f., Loiret, Eure-et-Loir, c. par M. J. Pо囚LEт.

coco jaune, m., Canisy (Manche), rec. pers.

foutagno, f., (c'est le mot obscène fouto avec un suffixe péjoratif), envir.

de Valence (Drôme), rec. pers.

celirlonia, italien, Florio, Worlil of words, 1611. - Sarl. du Surl, Moris,

Flora. - env. de Perouse. Zanetri, ?. 226.

celidonia maygiore, italien.

chelidonia, sarde logodourien, SPavo.

cinerognola, cenerognola magyiore, chelilonia maggiore, erba da porri,

erba da rolatiche, erbu marchesita, erba nocca, hirundinaria, italien, TARgioni, Diz. botan.

ceneraynuola, italien, DeE7, 1678.

celidonia maggiuri, sicilien, LAGusı, Erb., 1742.

cilidonia, sicilien. Cupani, Ilortus, 1696.

cedrogna, italien, Axgutlara, semplici, 1561.

erba zilidonia, zililonia, Parme, Malaspixa, Vocab.

celisionia, Aquila (Abruzzes), Fixamore, Botanica pop.

erlua dona, piémontais, Colla.

erba siriognia, erba siro, Bra (Piémont), Priocca (Piémont), Colda.

erba siula, Oviglio (Piém.), Colla.

siriögnia, erba siriögnia, prba li porèt, Piémont, ZAll, Diz.

siriognia, erba dii puret, Piémont. Annales de l'observat. de Turin, 1810 , p. 42.

erba sirogna, siracagna, Asti (Piém.), CamisoLA, [J. C.].

erba siriognia, Piémont, CAPELlo, Dictionn.

zeligogna, zerogna, Vérone, PoLluxi, Flora.

ceraddóneca, Celano Abruzzes. Fixayone, Botanica.

inzendonia, Trévise, Saccardo, flora.

erba donna, Tessin et Norare, Poldini, Flora.

erba döna, dôna, Valteline, Moxтs.

erba donnina, milanais, Bsxf, locab.

erba majestra, erba mormera, milanais, Cherubini.

erba maistra, Brescia, MelchioRi, Voc. bresc.

maistra, Côme, Moxti.

erba biignon, Alba (Piémont), Colla.

erba di por, Bologne, Coronedi-Berti, Yoc.

eiba dei porri, Calabre, La Calabria, 1894, p. 77.

erba zerra, Lanusei (Sardaigne), Moris, Flora. 
erba de zerras, erba de runas, sarde, Spano.

jerbe di Sante Polóne, celidónce, Frioul, PIrona, Vocab.

jarbe silidónie, f. sing., Udine, Pagine friulane, 5 agosto 1894, p. 104.

papagno selvaggio, Vésuve, Pasquale, Flor'a vesuv.

erba del lat zald, Brescia, Zensi.

siricayna, erba dona, environs de Turin, RE.

erba dentara, erba per $i$ ilenti, Padoue, PATRIARchI.

erba ca fa cruè $i$ dent, Asti (Piém.), Colla.

ciridueña, esp. du xve s., Dozy, Suppl. aux dict. ar.

celedonia, chilidonia, yerva de la golondrina, espagnol, Minsheu, Dict. 1623.

celidueña, golondrinera, espagnol.

cirigüeña, asturien, Rıтo.

celidonia, salidonia, Baléares, Marès, Cat.

selidonia, Catalogne, Varreda, Catalech.; Bull. de la assoc. d'excurs.

catal., 1889, p. 83 ; Costa, Flora.

erba de les oronetes, f., catalan, Lacavalieris, Gazophyl., 1696.

erba d'auranetas, Vall de Nuria (Catal.), Vayreda, Catal.

ceruda, galicien, Cuverno, Dicc. yall.

ciridónia, erba d'a anduriña, galicien, Valladares.

scänteiutça, roumain de Transylvanie, JARnik, Doine, 1885 (Gloss.).

scanteutsa galbana, rostopásta, rastopásta, roumain, CiнAc, Dict.

rostopasca, roumain, CuнAc, Dict.; BRANDZA, Limba botan., 1882.

ierba rîdunelei, nigelaritsa, roumain, BRANdzA, Limba bot.

rostopaste, jarbe rindunälie, roumain de Transylvanie, Fusz, Trivialna-

men.

rostopast, shintaitsa galbena, roumain, Pharmacopea româna, 1862.

sceliuurc, all. du XIe s., Mém. de la Soc. de ling., VI, 364.

scellivurz, ancien haut allemand.

scellewurze, anc. h. all., Germania, 1888, p. 303 et p. 304.

scellewurs, anc. h. all., Zeitsch. f. d. Alterth., 1843, p. 37 ก.

scehllewur\%, anc. h. all., Zeitsch. f. l. Alterth., 1872, p. 362.

sellewur\%, anc. all., PIPER, Altdeutsche Pflanzenn.

grintzwurtz, anc. all., DESCEMET.

goldwurz, schellewurz, gilbkraut, grintwurs, moyen haut all., DIEFENBACH.

schellwur\% (1), schöllkraut, augenkraut, schwalbenkraut, allemand.

schöllkraut, Carinthie, Zwanziger, Verz. - Basse-Autriche, Hörer, Wört.

(1) Par fausse étymol. pop. d'après Axdresen. Le mot se rattacherait originairement à Celidonia, latin clu moyen âge. 
schillkraut, Carinthie, LEXER, Kärnt. W.

schienfoot, Westphalie, LANdoIs, West/. Pfl.

warzenkraut, krätzenblumen, Carinthie, Zwanziger, Verz.

åfflkraud (herbe de l'érysipèle), Basse-Autriche, HöFER, Wört.

aflkraut, affelkraut, Carinthie, Zwavziger, V'er.

gilbkraud, liachtkraud, Basse-Autriche, HöFEr, Wört.

guhldkrockt, wuersekrockt, schellkrockt, allem. de Trausy̧lvanie, Fusz,

Trivialnamen.

hexenmilch, Alsace, Kirschleger, Flore d'Alsace, 1852.

giélzechskraut (= herbe de la jaunisse), goltwurzel, Luxembourg, J. WEBER. jülk, jölk, Altmark, DANNEiL.

schildlirut, schellchrut, gälbsuchtachrut, canton de Saint-Gall, WARtмıns. geschwulstkraut, Autriche, PRit7er, Pflanzenn.

truddemälch, Transylvanie, IDE.M.

nagelkraut, canton de Berne, IDEM.

blutkraut, Silésic, IDEM.

goldivört, Gœettingue, Schambacir.

schinnkraut, Prusse, Frischbier, $\mathrm{Pr}$. W.

goldwurzel, Eifel, Wirtgen, Vegjetat. d. Eif.

mel-kraut, allem. des Alpes rénitiennes, Schielder.

stinckende gouwe, groote gouwe, gouwortele, anc. flamand, DoDoweus,

Cruydt-boeck, 1644. [A. DE C.].

grote gouwe, stinkende gouwe, anc. néerlandais, DiefenBACH, Gl.

schellruid, ceciliadon, hollandais.

schelkens-kruid, stinkende youwe, oogenklaer, flamand, RoccEL, Flore, oogenlilî, Frise orientale, Focke, Vollisth. Pflanzenn.

celiadone, celialoone, salidone, flamand, DE Bo, Westvlaamsch Idiot.

[A. DE C.].

wrattenkruid, flamand, comm. par M. A. DE Cock.

teverwert, celethenie, celethonie, anglo-saxon, CockAyne, Leechd.

celandine, swallow wort, tetterwort $\left(^{\mathrm{l}}\right)$, silken sisley, anc. anglais, Fuorio,

World, 1611; Minsheu, Dict., 1623.

celidine, celidony, anc. anglais, HuLoEt, Dictionarie, 1572.

devil's milk, jacob's ladder, anglais dialect., Britten, Plant-names.

yarevye, irlandais, KE0GH, 1735. [H. G.].

lacha cheann ruadh (= red headed duck), irlandais, 0'ReILLY. [H. G.]

an ceann rualh ( $=$ the reddish head), aonsyoth (= lonely flower), gaélique écossais, CaMeron. [H. G.].

(1) From its curing tetters. (Prior). 
llys y wennol (= herbe de l'hirondelle), llys y llygaid (= herbe des yeux),

llyyadlym (= œil perçant), llygadlys (= herbe de l'œil), dilwydd

felen (= chélidoine jaune), gallois, Hugh Davies. [H. G.].

gwell nấ ' $r$ aur' (= meilleur que l'or), llysiau ' $r$ llaw (= herbes de la

main), gallois, J. WaLters. [H. G.].

luss-ny-pileyn, mannois, J. KELLY. [H. G.].

louzaouenn ann darvoed (= herbe aux dartres), louzaouenn ar guennelied

(herhe des hirondelles), breton du Finistère, c. par feu L. F. SauvE. sclær (mal imprimé silær), breton, Nomenclat.; P. GrÉGorRe. [E. E.]. sliler, breton, LEGONIDEC. [E. E.].

lousaouen ar güinnilied (= herbe des hirondelles), breton, P. GRÉGoIRE.

[E. E.].

lousaoüenn an daoulagad (= herbe des yeux), breton, P. GrÉGorRe. [E. E.]. lousaouen ou: an daoulagat, breton, Nomenclat. [E. E.].

skellört, flenört, svalört, tysk lima, nisse-i-luvars gata, suédois dial.

Jenssen-Tusch, Nord. Pl.

braenyrt, värteurt, gul müll, fnatblómster, danois dialectal, JENSSEN - Tusch. svaleurt, seladon, danois. [H. F. F.].

lirvavý kořen, tchèque, Šulek, Juy. im. bil.

vlastovičnil (de vlastovice $=$ hirondelle), nebesky dar, celidon, raupowé

kořeni, tchèque, A. Müller, Synonym. Namen.

celidon wétsii, wétši las̀towiënik, tchèque, PaLkowitscin.

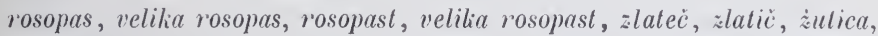
mrtvice, serbečica, rusa, trava od ruse, sula islirica, zomijino mlielio, slatni liorin, lastovičina trata, suta mličca, celadonija, celidomija, lirivljek, lrivośnili, yrenica, drasnica, lirivi zelje, lirirje mletio, liriunu mleku, rdec̈i mleček, rdeči mlečnel, bedemnica, cimbola, cindola, cindolica, cingola, serbo-croate, Šulek, Jug. im. bil.

jaslocze ziele, rostopaśc, zlotnik, polonais, $\mathrm{N}$ Еимісн, Lexic.

brodawka, brodawnil, polonais de la Prusse, Treichel, Poln. Vulgärn.

glistewnik, polonais, KozLowski.

brodawnik, kassoube, KozLowski.

dobrotnik, koliotowe mlolio, wende, Schulexburg, Wend. Vollisllnum.

tchistotiel, russe, Schmaliausen. [Th. V.].

tschistiak bolschoi, lastowitschnaya trawa ( $=$ herbe des hirondelles), russe, Falk, Beitr., 1786.

hletchkopar (') (= lave-pot), hlekopar, tchystotil (= qui purifie le corps), tcliystyk (= qui purifie), petit russien, c. par М. Тн. VoLkor.

(1) De hletchik = pot et de paryt' = vapeur. On lave les pots avec une infusion de cette plante. (VoLKov). 
tchystétze, kouriatcha slipota, Gouvernem. de Voronèje, c. par M. A. Dikarev. rostopastu, rostohvastu, petit russien, Ciнac, Dict.

akim, lithuanien, Mextzel, Lexicon.

gelton penis, lithuanien, ЈАсовт, Lit. Plunsenn.

struttenes, letton, STEXDEL, Lett. Ler.

sohbu sahles, saweeshu sahle, sarkanas strutenes, grawas strutenes, letton, Clyaxi, Lett. Wört.

enudabelar'u, basque. [j. V.].

veréhullofii, maģvar, Fusz, Trivialn.

nag! fetslie fü, cะinedonia, magyar, Neмsich.

algir, arabe, SÉlAPIOx L'AxcıEx, Practica, 1525.

o'rouk es sabbighin (= racines des teinturiers). chedjret el lihalatif, arabe, lbx Beithar (Edit. Leclerc), II. p. 320 et $\mathbf{4 1}$.

lebaïna, a rabe algérien, Flonax P'HaRaоx, Voc., 1866.

khâlidounioun, maramîram liélur !'), arabe syrien, BergGrex, Guide fr. ar.

chirlanctizu otu, turc, Pharmacopea romana, 1862.

zerde tchiop, persan. Mexisski.

daidsan daaghik (= lleur des hirondelles), dridsran chot ( $=$ herbe des

hirondelles, dzidsérnadègh $=$ médicament des hirondelles), disilzérnaki dègh (= médicament des hirontelles ), deghin cotch, kantheghchot (= herbe de lampion), arménien, Alishax. [Er. L.].

kousano oou = reine des herbes), chinois, Horfus.x.

scukure: langue inconnue du XII siècle dans la région de Wiesbade, DESCEMET.

2. On attribue une grande efficacité i cette plante contre les maladies d'yeux.

«Quand une taie se forme sur les yeux, il faut, pour l'empêcher de s'étendre et la faire disparaitre, bassiner fréquemment l'organe malarle avec de l'eau de pluie, dans laquelle on a mis it tremper, pendant louze heures, une poignée de feuilles de chélidoine. »

Finistère, comm. par feu L.-F. Sacvé.

«. Arez-rous une taje dans l'œil? Prenez un pied de grande éclaire, appliquez-le sur le poignet et dites une neuvaine, la taie s'en ira. 》

Pays bulonnais, E. DEseille, Curios., p. 107.

“ Des aninaux aussi ont décourert des plantes et entre autres la ché-

(1) Comparez la forme latine du VII s. ap. J. C. mamira donnée par AlexandRE DE Tralles, Pratica, 1520, feuillet 43 , recto, qui est certainement dorigine orientale. 
lidoine. C'est avec cette plante que les hirondelles rétablissent la vue de leurs petits daus le nid, même, assurent quelques-uns, quand ils ont les veux crevés.

PliNe, Hist. nat., XXV, 50 (Trad. Littré':

"Chelidonia ideo dicitur, vel quod adventu hirundinum videtur erumpere, vel quod pullis hirundinum si oculi auferantur, matres eorum illis ex hac herba mederi dicuntur. " IsIDORE, Etym., XVII, IX, 35.

"Prima si dice che il succo di essa, per esser acre e corrosivo, distrugge i porri e percio si usa comunemente. Lo stesso succo si adopera come purgativo. $\mathrm{E}$ in fine si tiene efficace per rischiarare la vista. I contadini dicono che quest' uso l'hanno imparato dalle rondini, le quali si serrono di quest' erba per ridonare la visita a' loro pulcini. »

Bologne, C. Coronedi-Berti, Appunti de medicina popol. bolognese, 1878.

"Dic Schwalben geben ihren Jungeen, so lange diese blind sind, die Blætter zu fressen, damit sie um so schneller sehend werden. »

Canton de Saint-Gall, Wartmans, Volksbotunik.

L'hirondelle nous apprend l'experience de cette herbe, car quoi que l'on ait crevé les yeux à ses petits, par le moyen de cette herbe elle leur renı la vùë, aussi elle fleurit lor's qu'elles arrivent, et flétrit quand elles s'en vont.

II ${ }^{\mathrm{me}}$ Fouguet, Suite du recueil de remèdes, t. II, 1701, p. 396.

3. Le suc de la tige de cette plante est d'un beau jaune; on s'en sert dans plusieurs contrées pour guérir la jaunisse. similia similibus curantur.

«Contre la jaunisse, prenez de l'herbe de chelidoine, mettez la daus ros bas ou souliers, et faites en sorte qu'elle touche le pied nud, changés-en tous les jours et vous guérirez. »

$\mathrm{M}^{\mathrm{me}}$ Fouquet, Suite du recueil des remèdes, t. II. 1701, p. 189.

4. Les feuilles de cette plante, en cataplasmes, sont employées contre les panaris; réduite en jus, elle est un remède contre les vers intestinaux.

Haute-Garonie, comm. par I, P. Fagot.

En Flandre, comme en beaucoup d'autres pays, on emploie le suc de la chélidoine, pour détruire les verrues; de là le nom populaire de la plante Wraltenkruid; on s'en sert encore, sous forme d'infusion de la racine, pour guérir le lumbago.

Commun. de II. A. de Cock.

Voyez dans Feller, p. 292-993, d'autres usages médicaux populaires de cette plante, dans le Pays wallon.

5. In plantaire du moyen âge, intitulé Poème moralisé sur les propriétés des choses, publié dans la Romania, 1885, par M. G. Raynaud, consacre 32 vers aux vertus de cette plante. 


\section{HYPECOUM PIROCUMBENS. (LINNĖ).}

نंTr火oov, grec ancien, D10scoride.

cuminum sylvestre, cuminum siliquosum, cuminum corniculatum, anc.

nomencl., Bauhis, Pinax, 1671.

cumin cormu, m., français, NЕмхıн, Lex.

cumi cornut, m., Pyrénées-0rient., Companyo.

coourun ménu, m., Var, Haxrs, Cat.

zadorija, pamplina, espagnol, Nevicu, Lexicon.

pampline, Baléares, Marès, Cat.

karn el mo'aza, Malte, Delicata, Flora melit.

\section{HYPECOUM GRANDIFLORUM (Втн.).}

ballarida, catalan, Costa, Flora.

\section{FUMARIÉES.}

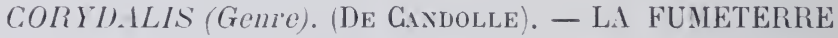 BULBEUSE.}

\section{1. - NOMS DE LA PLANTE,}

fumaria bulbosa, fumaria phraymitis, capnos chelidonia, capnos phraymitis, chelidonia phragmitis, wistolochia phragmites, aristolochia rotunda, anc. nomencl., Batus, Pinax, 1671.

aristolochia cal'a, anc. nomencl., Rosexthar, Synopsis plant. diaph.

fumaria cava, corydalis cara, corydalis bulbosa, corydalis tuberosa, nomencl. de divers auteurs.

fumeterre bulbeuse, f., français savant.

crête de coq, f., Anjou, Desvaux, Flore.

bec d'oie, m., ivrogne, damotte, f., Doubs, Beacquier, Voc.

soupe en vin, f., poulette, f., jacinthe sauvage, f., Montoy (Pays messin),

c. par feu AURICoste de LAZARQLE.

pain sans vin, m., Coincy (Pays messin), IdEI.

panvin, m., Vigy (Pays messin), IDEu.

fummosterno bulboso, italien.

violeta bulbosa, espagnol, Neмxich, Lex.

froles cordeales, galicien, Cuverro, Dicc. 
fumaria bolbosa, portugais, Neunich.

brebener, ruumain, Brandza, Limba bot.

aluné, f., alunele, f. plur., roumain, Сінас, Dict.

halewur\%, hellewurz, moyen haut all., Pritzel, Pfl.

knolliger erdrauch, runde hollwurzel, hersururs, frauenschnh, tanben-

kropf, löwenmaulerl, farnsamen, donnerfluch, allemand, Хемісі. lerchenspor'n, haberkraut, haselrausch, höhnele, burgerschlïssel, wutte-

bïschlan, zottelhose, hosenzottel, hosenzottele, Carinthie, ZWaxzigEr. hohlwurzel, bibahend, Autriche allem., HöFER, Wört.

baumchenhohlwul'zel, Prusse, Frischbier, Pr. Wört.

jerdapelcher, Transylvanie, Pritzel, Pfl.

rothe henne (Corydalis bulbosa ì fleurs rouges), Zillerthal (Bavière),

Grimi, Wörterb.

weisze henne Corydalis bulbosa à lleurs blanches), IDEM, idem.

rossthräni, Lucerne, Pritzel, Pfl.

schlösselbluama, frauaschlössel, buchs, gügarüyu, schlïssel, canton de

Saint-Gall, Wartмasx, lolksb.

wiessi und rothi hoesele, guli c.-à-d. le coq, la variété à fleurs rougges),

henna (c.-it-ll. la poule, la variété à fleurs blanches), cant. de Saint-

Gall, Wartuaxi.

rösli, Oberland bernois, PRITZEL, Pllanzennamen.

giggerihaner, Tyrol, IDEM.

splith, ancien flamand, Dodoraeus. [A. DE C.].

helimbloem, hollandais, OcDEMANS. [A. DE C.].

leeuwerikbloem (= fleur aux alouettes), flamand, Vax Heurck et Guberr,

Flore medicale belye. [A. DE C.].

boontjes-holwortel, vogfeltjes, flamand, DE GORTER.

holleac, anglo-saxon, Cockarve, Leechd. 1866.

hollow-root, hollow wort, anglais.

gulhanar, m. plur., suédois dialectal., Rietz, Stenslit dialect-lexicon, 1867.

hölört, nunnört, suédois, JENSSEN-TUSCH.

hulrod, roed hanekam, danois, JENSSEN-Tusch.

riast bily, piunyli ( $=$ petits coqs), petit russien, c. par М. Тн. VoLkov. dymirlia, dymuivka, tchèque, A. MëLler, Syn. Num., 1866.

mladja, mladjak, brezroda, zinijska trava, kapulota, peteliniek, golnlija guša, mačja larfulica, serbo-croate, ŠulEK.

tavaszi gere deske, lyikas-ir, magyar, NЕмхıсн, Lex.

2. Le Corydalis a la propriété d'empêcher la pousse des cheveux. Quand ceux-ci descendent trop bas sur le front ou qu'il pousse des poils entre les 
sourcils, les femmes les arrachent et frottent l'endroit avec le suc de la plante.

Dodonaeus, Elit. de 164t' (A. DE C.).

3. « Midchen stecken die zufällig gefundene Pflanze in der Busen, dann begegnet ihnen der zuliüntige Geliebte. 》

Necklembourg, Lisch UNd Beien, Mecklemb. Jahrb., 2(1, 186.

\section{CORIDALIS LUTEA. (DE GaNdoLle).}

fumaria lutea, fumaria corydalis, corydalis, anc. nomenclature, BuUnis, Pinax, 1671.

fumeterre jaune, f., français.

sprit, ital. du XVIe siècle, J. Canus, Itistorique des herbier's, 1895, p. 25. laty's pincushion. mother of thousands, Devonshire, Friend, Giloss., 1882. yellow fumitory, anglais.

FU.MARIA OFFICINALIS. (LINNÉ), - LA FUMETERRE.

\section{1. - NONS DE LA PLANTE.}

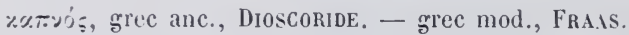

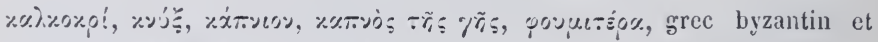
mod., LAXgKaYel, Gesch. d. B.

«ятvi\%, grec mod, P0lQueville, Voy. - gr. mod. d'Argolide, SibThORP.

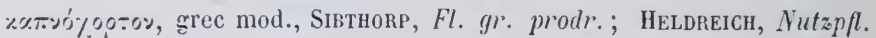

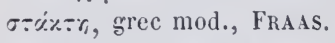

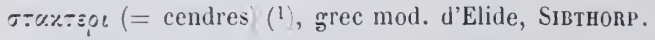

жоviт:р\% (2) grec mod. de Laconie, SirthorP.

corydalion. grec mod., Axgunlara, Semplici, 1561.

fumus terra, fumaria, capnos, fumulus, nomencl. du moyen ảge, Mowat. cumusella, nomencl. du moyen àge (...i ${ }^{\mathrm{e}}$ s.', Bibl. de l'Ecole des Chartes,

1869. p. 331 .

capnion Dioscoridis, nomencl. anc., J. AGRICoLA, Med. herbar., I539.

(1) Weil zum Aschenbrennen mit Salsola und Atriplexarten verwendet (FraAs.).

(2) Selun FraAs ce nom doil sappliquer au Corydalis. Ce serait une erreur de SibThorp. 
fumus terrestris, lat. du moyen-âge, Germania, 1888, p. 304.

fumus terrenus, latin du xin ${ }^{\mathrm{e}}$ siècle, Aegidii carmina medica, édit. Choulant, 1826, p. 202.

fumiterra, fumitara, herba fumida, latin du mojen ìge, Diefenbach, Gl. fumaria, t., Nice, Risso, Hist. nat.

fumorèl, m., Le Buisson (Dordogne), rec. personnell.

fumichon, fimichon, Eure, RoBs, Dict.

fum'ron, m., Ruffey (près Dijon), rec. personn.

fumaterra, f., Pyrénćes-0rient., Coupdiro. - Gard, l'ouzoLz, Flore. Montpellier, LORET.

fumoterro, f., Var. - Bouches-du-Rhòne. - Basses-1lpes. - Drône. - Gard.

- Corrèze. - Aude. - Gers. - Lot. - Lot-et-Garonne. - Haute-Garonue.

- Cantal.

fumeterro, f., Apt Vaucluse), Colig.ron.

fumiterre, f., anc. franç, L'Arbolayre, 1490.

fumeterre, f., anc. franç. - français mod. Le mot est quelquefois masculin). feumeterre, m., Saint-Pol (Pas-de-Cal.), c. par I. Ev. Edroxt.

femeterre, fumeticire, Vosges, Halllant, Fl. pop.

fumrotèro, f. Eymoutiers (Haute-Vienne), rec. personn.

frumterre, Spa, LEzıACK, Fl. - Malmédy (Pays wallon), Feller.

fineterre, f., wallon du $15^{\text {e }}$ siècle, J. CAsus, $\ln$ manuscrit namurois. Vosges, Haillant.

funterre, anc. français, Reissch (dans Archiv. de Hernig, 1830, p. 175.

ahumada, f., (= enfumée), Luchon (Pyrénées), J. Sicaze.

fournayo, f., env. de Valence (Irôme, rec. personn.

fournandière, f., P'onts-de-Cé Maine-et-Loire', rec. pers.

fiel de terre, nı., français.

féou dé terra, m., Le Vigaı (Gard, Rouger, Top. - Gard, c. par II. P.

Fesquet.

héou, m. (= fiel), Luchon Pyrériées), J. Sıcaze.

fiô de tarrè, m., Issoire (Puy-de-Dòme, c. par feu J. bareire.

tarribustelo, f., Saint-Saturnin (Provence), CAstor, L'Interpr. pror'.

tarribusteri, terribusteri, Bouches-du-Phône, Vhlenecve, Stat. - Avi-

gnon, Palcs, Cat.

soupe au vin, f., Aube, Des Etanggs. - Yonne, c. par II. Eys. CRÉdÉ.

trempée au vin, f., Iube, Des Etangs, Noms pop., 184.

trempee aux oiseaux, f., Aube, Des ETANgs. - Haute-llarne, c. par

II. A. Daguin.

trempée aux vaches, f., herbe aux dindons, f., Aube, Des Etangs.

pa d'aouci (= pain d'oiseau, m., Velay, Mistral.

herbe aux cochons, f., Semur-en-Auxois (Còte-d'Or), c. par M. H. Marlot. 
erba de colom, f., Pyrénées-Orientales, Companro.

pain aux souris, m., Mayenne, comm. par un botaniste de la Mayenne. chaude souris (= chauve-souris), Saint-Brice (Ille-et-Vilaine), rec. personnell.

ubriago, f., environs d'Aix en Prov., Gakinel. - Apt (Vaucluse), ColiG.von, Fl. - Var, Hanri, Cat.; Amic, Consid.

embriago, f. provençal moderne, RéGuis.

raisinetle, f., Charente-Infér., comm. par M. E. Lemarié.

tendresse, f., Ineuil (Cher), rec. personnell.

bonhomme grille, m., Ille-et-Vilaine, Orain, Gloss. pat., 1886.

crête de coq, f., be'c d'oiseau, m., bec d'alouette, m., Normandic, Joret. pied de yéline, m., anc. franc., Pixeus, Hist. pl., 1561.

pato dé poulo, f., env. de Barbaste (Lot-et-Garonne.), c. par M. l'abbé L. DARDY.

faouterno, f., Gard, c. par M. P. FesQueT.

futerne, f., Vendée. LALANne, Gl. lu p. poit.

sarrasine, f., Charente-Infér., comm. par M. E. LExarué.

vigogne, f., cricraihe, f., demoiselles, f. pl., Normandie, JonEt.

giverlan (de giverd = persil, avec le suff. an), mentonais, ANDREWs.

tchaissenotte, f., Montbéliard, Contejean, Gloss.

fenouhieiro, f., Gard, c. par M. P. Fespuet.

sany-mélure ( $\left.{ }^{1}\right)$, f., Centre de la France, Jaubert, Gl.

fruit de terre, m., Val ite Saire (Manche), JoRet.

fleur de terre, f., Pays de Caux (Seine-Inf.), Joret.

li de terre, m., canton de Périers (Manche), rec. personn.

petite lirelte, f., Coetmicux (Cötes-du-Nord), rec. personnell.

cuiz cenviz, français du xiı siècle, Biblioth. de l'Ecole des Chartes, 1869, p. 331 .

fummosterno, anc. ital., Mesue, Libro della consolatione, 1475.

fumara, fumoterra, ital., Lexicon italico-latinum, 1648, p. 111.

fumaria, fumosterno, italien.

erba fumaria, Parme. Malaspina, Voc. - Fabriano, Marcoaldi, Guida de

Fabriano, 1877. - Mantoue, Cherubini.

erba da purghe, italien, TARGION.

fiele della terra, erba calderugia, erba acetina, feccia, pie di gallo, pie

di gallina, formes italiennes mentionnées par Casaccia dans son

Dictionn. gênois.

fümmisterno, erba fümmajêua, Gènes, CAsaccia, Diz. gen.

(1) Cettc plante passe pour avuir la propriélé d’activer la circulation du sang. (JaUBERT). 
fumu sarvaggiu, pitrusinu sarva!ggiu, île d'Ustica, Calcara.

foem, Brescia, Zersi.

fumeria, romagnol, Morri.

foemeria, foensteren, Brescia. MELCHIORI, Voc.

fumjiera, Abruzzes, Finamore, Bot. pop. alm.

fumaria rossa, Vésuve, PASQUale, Fl. ves.

fumisterra, Sardaigne du Sud, Monis, Flora.

fumister, Sardaigne, Porru, Diz. sardu.

fumusterru, sicilien, BIANCA, Fl. d'Atola.

fumusternu, sicilien, LAGUSi, Erbuario, 1742.

fumoterra, napolitain, Savastanus, Botanicorum libri, 1712, p. 19.

cambirugja, Macomer (Sardaigne), Moris, Fl.

fumusterre, cambirija, sarde logodourien, SPANo.

carabinazzi, Vérone, PolliNi, Fl. ver.

siringa sarvaja, Annone (Piémont), Felissano (Piém.), Colla.

erba seren-a, Vignale (Piém.), Colla.

palomina, espagnol, Cuusius, Rar. plant. hist., 1601.

yerba palomina, esp., Asovso, Privil. para mugeres, 1606.

palomilla, espagnol, Pamirneno, Vocab., 1575.

yerba palomilla, esp., Alonso, Priv. p. muy., 1006.

filomosterra, esp., Oudin, Tresor, 1660.

palomillo, conejitos, fumaria, espagnol.

gatico, Espagne du Sud, BoIssier, Voy.

erva molarinha, anc. portugais, Barb0sa, Dict. lus., 1611. - portug.

$\bmod$.

fumaria, fumiterra, fumo da terra, erva moleirinha, portug.

erba dona, galicien, Cuverro, Dicc. gall.

fumusterra, catalan, Agustin, Secretos d'agricult., 1646. - Baléares,

Marès, Ciat.

fumisterris, catalan, LACAVAlleria, Gazoph., 1696.

fum de terra, catalan, Bull. de la Associac. d'excurs. catal., 1889, p. 83.

jaubert brut, Baléares, Marès, Cat.

fumarica, saftiré ( $\left.{ }^{1}\right)$, corcodan, roumain, Сıнас, Dict.

fumaritsa, saftire, saftérea, saftéora, roumain. Brandza.

jarbe de kurke, fumu pomuntulur, roumain de Transylvanie, Fusz.

ertrouch, bockespart, taubencropf, anc. haut allem., Germania, 1888, p. 304 .

fumusterre, fumester, anc, allem. du Nord-ouest, GallÉE.

(1) Mot d'origine turque. 
roek, moyen haut allem., Pritzel et Jessen.

katzenkerbel, katzenklee, kat:enklauen, nonnenkrult, bocksbart, moven haut allem., Diefenbach.

erdrauch, ackerraute, erdkraut, grünwurะel, krälzheil, alpraute, allemand, Nемnјсн.

feldraute, ackerkraut, wilderaule, taubenkropf, taubenkörbel, kat:enkorbel, grindlraut, nunnenkraut, fimstern, allemand, Вїнмек, Comment. accon. med. bot., 1792, p. 58.

kâzelîrwel, dauwekirwel, râchkraut, Luxembourg, J. WEBER.

nunnenkirut, Goettingue, Sснамвасн.

taubenkerbel, Eifel, Wirtgen, Veg. d. Eif.

vogelkraut, Grosbliderstroff (Lorraine), rec. personn.

wildes weinkraut, Carinthie, Pritzel, Pflanzenn.

fimstart, brulkraut, Mecklembourg, Pritzel.

apostelkraut (1), fälsche weinrutn, Basse-Autriche, HöFER, Wört.

fünstern, Prusse, Frischbier, Pr. Wört.

fül grêt, lewkenkruil, Altmark, DanneIL.

jerdrûch, all. de Transylvanie, Fusz.

schone vrowe, scoene culte, moyen bas allem., Dierenbach, $\mathrm{Gl}$.

feldtroock, gryscom, anc. néerlandais, Diefenbach.

duyvenkervel, grijsecom, anc. tlam., DodoxaEus, Herbarius, 1608.

aardrook, duivenkervel, hollandais. [A. DE C.].

grijzorie, grijzekom, kraaibek, flamand, DE Bo, Westvl. Idiot. [A. DE C.] roode remke, Groningue, DE VRIEs, Woordenboek, 1866. [A. DE C.]

duive-kervel, aard-rook, flamand, DE GORTER.

koe-gras, Maestricht, DE GORTER.

cuntehoare, anglo-saxon, EARLE, Engl. plant-names, 1880.

cunthaere, anglo-saxon, Соска хxe, Leechd., 1866.

fumitory, anglais.

fuimiterra, moyen irlandais, STOKEs (dans Revue celt. IX, 234. [H. G.]. mantenaigh, serag lalhum, cummansharr'y, irlandais, KEOGH, 1735.

[H. G.]

fuaim an t-siorraigh, $\left({ }^{2}\right)$ irlandais, 0'Reili.y. - gaélique écoss., Cameron.

[H. G.].

cuman scarraigh, maintenagh, irlandais, THRELKELd. [H. G.]

(1) In den an einer Flanke senkrecht übereinanderstehenden Blüten erkennt (auch der Zahl nach) unser Volk eine Hindeutung auf die zwoelf Apostel.

(F. HOEFER)

(2) Cameron traduit le mot irlandais par "fumée du sheriff " et pense que c'est une traduction plaisante de Fumaria officinalis (officer's fumaria). 
deatach thalmhuin, (= fumée de terre), irlandais, Cameron. [H. G.]

lus deathach thalmhainn, dearag thalmhainu, gaélique écossais, CAMERoN.

[H. G.].

tudfwg (= fumée de terre), gallois, J. MoRGan. [H. G.]

mwg y dilaear, (= fumée de terre), gallois, Hugh Daries. [H. G.].

cuid y mwy, (= sac de la fumée), gallois, J. Walters. [H. G.]

booa-ghoayn, mannois, Cregheen. [H. G.]

sawellys, (= plante du trou de la fumée), gallois de Llanrwst, J. Williass.

[H. G.]

flemmeterr, breton, Nomenclator. [E. E.].

flémineter, breton, P. GRÉGoire. [E. E.].

fémm-douar, breton, P. GréGolke. (Le mot signnifierait « aiguillon de terre »

selon LE GONIDEC, mais c'est uı arrangement populaire du précédent.)

[E. E.].

mogueden-douar (= fumée de terre), breton de Vannes, P. GrÉgolre.

[E. E.].

louzaouenn ann teil (= plante du fumier), breton, Trouve. L'auteur ajoute,

« ce nom, dit-on, lui vient de ce qu'elle laisse dans la bouche un très

mauvais goût quand on la mâche. » C'est plutôt une imitation du fran-

çais fumeterre pris au sens de fumier de terre). [E. E.].

jordrök, aikersille, jungfru Marie sünghalm, bukarfe, gallyras, rökniv,

suédois dial., JENSSEN-TusCH.

jord $\not y$, karetuogn, danois, JENSSEN-TUSCH.

dimica, dimljača, dimnjača, dimnjavica, zemaljski dim, rusnica, rosnica, rosica, rosopast, rusno zelje, rosno zelje, pelinac, mali pelinak, runjavac, liljuć svetoga Petra (clef de Saint-Pierre;, kljucić svetoga

Petra, mačja hrfulica, serbo-croate, Šulek.

polni routa, plana routa, routickla, zemêllym, tchèque, A. MüLler, Synn.

Nain.

rutka polnj, tchèque, Роцкоwiтsch.

kokorycz, kokorzyk, polonais, LINDE.

dymianka, (= plante de la fumée), russe, Schmalmausen. [Tlı. V.]

tschistak, russe, GEORGI.

poleva routa (= rue champêtre), ptachyna routa (rue des oiseaux), petıt

russien, c. par M. Тн. VoLkor.

mahtes sahle, letton, STENDER, Lett. Lex.

negakina, basque. [J. V.]

emina tus, emma rohi, punand, esthonien, HUPEL.

földfustfui, magyar, Fusz.

fom, albanais, Heldreich, Nutzpfl.

schateradj, arabe, Freytag. 
shatredj, arabe algérien, Jourdan, Flore murale, 1867.

shahtreg (espèces voisines du Fumaria officinalis), arabe égyptien, Ascherson et Schweinf.

shâterre, persan, PoLak, Persien, 1865.

shih outroudj, zoueyté, arabe syrien, BERgGren, Gride.

baglat ul mélik, arabe, Barbier de Meynard, Dict. turc.

liousfaratoun el hhimàr, (= coriandre de l'âne), arabe, SanguinetTi,

Chap. de méd. arabe, (Journ. asiat., 1868.)

dolihanet l'art, Malte, Dflicata, $\mathrm{Fl}$. mel.

tfishe, arabe algérien, MunBr, Noms arabes des plantes.

sibân, guerin djedey, arabe algérien, Duvelrier, Les Touaregs, 1864.

shahtharak, andzchot, arménien, Alishax. [Er. L.].

shìhteré, turc, Сıнас, Dict.

siahteru otu, turc, Pharmacopea româna, 1862.

pitpapra, pitpara, hindoustani, Fallov.

2. La Fumeterre est ainsi appelée pour ce que si on met son suc sur les ycux pour les esclarcir, à quoy elle a grand efficace, elle excite les larmes, tout ainsi que la fumée...

A. Constantin, Brief traicté de la pharmacie provinciale, Lyon, 1597, p. 117.

Fume terve est une herbe que l'on appelle ainsi fumus terre, pour ce qu'elle se engendre d'une grosse fumosité qui se esliesve de terre, et aussi qu'elle yst de terre en grant quantité ainsi comme fuméc.

xvo s., J. Camus, L'Opera salernitana, p. 69.

L'origine du mot fumus terræ est controversée. Voyez à ce sujet BRITTEN, Plant-names, sub verbo fumitory.

3. Sparisce come l'erba fumaria. (ll s'est éranoui, il a disparu comme la fumeterre.) Proverbe de Fabriano, Marcoaldi, Guida della citta di Fabr., 1877.

4. Les paurres recueillent cette plante et la fument mélangée avec le tabac.

Soulaucourt (Haute-Marne), c. par M. l'abbé Marchal.

5. Un bouquet de cette plante placé extérieurement à la fenêtre d'une jeune fille indique symboliquement que le mal, qu'on a dit d'elle, est vrai. Il n'y a pas de fumée sans feu.

Ruffey (près Dijon), rec. personn.

\section{FUMARIA GAPREOLATA. (LINNÉ).}

iбótupor, grec anc., Dioscoride (selon FraAs).

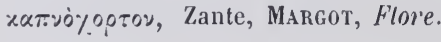


fumaria bianca, Vésuve, Pasquale, Flora vesuv. - Mondovi (Piémont), Colla.

conejitos de los vallados, espagnol, CoLmeiro, Dicc. de los nombres. erva molarinla maior, portugais, BRoTER0, Flora lus. sibrin, arabe tunisien, Prax.

\section{FU.MARIA AGRARIA. (Lagasca).}

yuemalli-bellardj, ragma, arabe de Constantine, Prax.

\section{PLATYGAPNOS SPICATITS. (BERNH.)}

fumaria spicuta, nomenclat. de LivNĖ.

terribusteri, Apt (Vaucluse), Colignon, Flore.

maou de testo, m., Avignon, Paluv, Cat.

\section{BOCICONZA FRLTESCENS. (LINNÉ).}

celandine, parrot weed, anglais colonial, CrRISEBACH.

\section{DIELYTRA (') SPECTABILIS. (DE GANDOLle).}

cœur de Marie, m., diclytra, m., français.

Reur de Marie, f., français, Mall.

Saint-Esprit, m., Saint-Pol (Pas-de-Calais), comm. par M. Ed. Eduont. baiyneuse, Enfant-Jésus au berceau, envir. de Paris, rec. personnell. boucles d'oreille, f. pl., environs de Metz, rec. personnell. gebrochen herz, jungfern-her\%, Marien-herz, bambel-herzche, Nassau, Geisenhagner.

hars blome, poppenblome, Oberneuland, Focke.

hersblumen, Carinthie, Zwanziger. - Prusse occident, Treichel.

bisschoppen (= évêques, kabasjesbloem, Flandre orientale, Pays d'Alost, comm. par M. A. DE COCK.

gringellen, gringelletjes, flamand, DE Bo. [A. de C.]. sweet betsies, locks and keys, West Somerset, ELWовтнy, West Somerset Word-book.

love-lies-bleeding, Devonshire, Friend, Giloss. of Devonslire Plant-Names.

(1) Par suite d'une erreur, qui se retrouve dans la plupart des ouvrages de hotanique, cette plante a été à tort dénommée: Diclytra. - Le $c$ doit être remplacé par un $e$. 
lady's ear-drops, lady-in-a-boat, anglais des Etats-Unis, BERGEN. hjärta fröjd, Suède.

hjœrteblomst, hyrdehjœrte, färe-hjœrte, danois.

pantofelki Matki Boskiej (= pantoufles de la Mère de Dieu), serduszka (petit cœur), polonais, comm. par M. J. DRĖGE.

Wenn man deren Blüthe einem Mädchen zeight, so sagt sic :

Wat de domme Jong sich denkt?

He denkt, ick heww min Hart verschenkt!

Ne, ick heww et nich verschenkt,

Heww et an den Strûk gehängt!

Neustadt (Prusse occident.), TreicheL, Volksth. \IX, 1874, et Volkslieder aus Westpreussen, 1895, p. $1 \dot{\sharp}$.

\section{GRUGIFERES}

morettia PHilaEANa. (De Gindolle).

saggar, arabe égyptien, Ascherson et SCHWEINF.

\section{MATTHIOLA INCANA ('). (R. BROWN). - LA GIROFLÉE}

\section{DES JARDINS}

1. - NOMS DE LA PLANTE.

šrsoị, grec ancien, ThÉophraste, De causis plant., IV, 25 ; identification assurée par Eugène FourxiER, dans son travail Sur l'étymologie et les origines du genre Hesperis (dans Bullet. de la Société botanique, 1866).

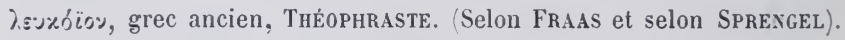
$\sigma_{\llcorner} \dot{\lambda} \alpha$, grec moderne, FraAs, Synopsis plant.

(1) Il y a deux variétés de cette plante, l'une d'un rouge vif, l'autre d'un beau blanc. 
viola alba, latin, Pline, XXı, 6, 11; Columelle, IX, 4; X, 97 (selon FraAs).

pallens viola ( $\left.{ }^{1}\right)$, latin, VirgiLe.

leucoia varia, nomencl. du $16^{\mathrm{e}}$ siècle, Max ScнмidT.

viola candida, viola alba, viola matronalis alba, viola matronalis pur-

purea, leucoium album, leucoium purpureum, leucoium incanum,

ancienne nomenclat., BadHix, Pinax, 1671.

cheiranthus incanus, nomenclat. de Linsé.

hesperis violaria, nomenclat. de LAMARCK.

giroflée, f., giroflée des jardins, f., violier, m., français.

giroflée grosse espèce, f., français, Bon jardinier p. 1827; Rev. hortic., 1850,

p. 18; Catalogue de Vilmorin, 1894.

grosse giroflée, f., français, Revue horticole, 1850, p. 17-18.

giroflée rouge, f., Allier, comm. par M. E. OLIviER.

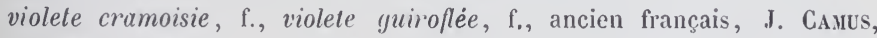

Livre d'heures.

vioulié d'estiéou, m., provençal moderne, RÉGuis.

gérouflée, f., gérouflie, f., giroufie, Bocage (Normandie), JoREt, Fl. p.

ginofrèe, f., Saint-Pol (Pas-le-Calais), c. par M. Ed. Edmont.

garaniè, m., Aix en Provence, BOYER DE Fonscolombe, Calendrier de

Flore, 1845 .

garranie, m., Bouches-du-Rhône, Villeneuve, Statistique des Bouches-

du-Rhône.

gouranié de tout l'an, m., provenç. mod., RéGuls.

goouraniè dé jardin, m., Var, Hankr, Catal. des pl.

bigouille, canton de Saint-Germain (Lot), Soulué, Catal. des pl.

biouléy', m., Libourne (Gironde), c. par II. L. DuRANd-DÉGRANGE.

bioulie, m., bioulié caranten, m., sud-ouest du Languedoc, Duвoul.

bieille, m., La Roquebrou (Cantal), c. par M. J. MaLvezin.

bioulè, m., biouliè caranten, m., languedocien, Duboul.

brioulè, m., env. de Barbaste (Lot-et-Gar.), c. par M. l'abbé L. Dardy.

vioulié rougé, m., Villefranche de Lauraguais, c. par I. P. FaGoT.

barico, m., mentonais, ANDREWs, locab.

violacciocca rossa, fior barco, leucojo bianco, violacciocca bianca, viola rossa, viola bianca, ital.

violacciocco, violacciocco rosso, violacciocea grigiolata (= Matth. flore

(1) BENoIst (dans son Virgile, I, p. 19 en note) veut voir dans pallentes violae les giroflées jaunes, mais pallens signifie blanc et non pas jaune. 
varieg.), violacciocca pavonazza (= Matth. flore purpureo), violacciocca pallida, italien, TARgIONI.

scrotina viola, italien, Duez, Diz. ital.; 1678.

vivole a ciocche bianche, ital., TARGION.

barcu, fior barcu, sicilien, Büsul, 1856.

baichi, Gênes, DE Notaris.

bàlacu, sicilien, Mortillaro.

violetta italianu, italien, Lericon italico-latinum, 1648, p. 107.

viola arrubia, Sardaigne du Sud, Monss, Flor'a sarloa.

aleli encarnado, aleli encanecillo, aleli blanco, espagnol, CoLmejro, Dicc.

de los nombres.

violetta blanqua, espagnol, Dodoess, 1608.

violer vermell, catalan, Costa, fil. de Cat.

goveiro encarnalo, portug., Brotero, Flora lusitanica.

aleli, galicien, Cuvejro, Dicc. fjall.

viora roshie, micshunica, roumain, Brandza, Limba botanica.

musliatenblümle, anc. h. all., Max Scumid.

winter-leutioje, stockieil, allemand, Rosentuld, Synopsis plantar.

gartenveiel, stochviole, allem., NEunich, Polyglolten-Lexicon.

leucoie (la fleur), leucoienstock (la plante), allem., Poeteris, Nouv. dict.

all., 1754 .

sommerfeigel, Carinthie, Zwanz.ger, Verzeichn.

levkuv, Aix-la Chapelle, Weitz, 1836.

leefloje, leefflioje, Vallée de la Weser inférieure, Focke, I'olksth. Pflanzenn.

vigeletten, Prusse, Treichel, Vollisth. aus d. Pflanzenwelt.

wies:apfa, maiazapfa, strossburgerli, pfingstveiali, veali, stammanägeli,

wiessmanna, canton de Saint-Gall, Wartmans, Saint-Gall. Volksbot.

lamberti veigel, Autriche.

lamperta, Vienne, LoRitza, 1847.

stock gilliflower, stock, anglais, PRIOR, Pop. names of brit. plants.

hopes, Norfolk, Britten, Dict. of engl. plant. names.

yernsey violet, anc. angl., Gerarue, 1636.

yarnesie violets, castle gilliflover, anglais dialectal, Britten, Idem.

leviriij, suédois et danois.

lewlionia, polonais, Linde, St. jez. polsk.

rumanija, serbo-croate, Šulek, Jug. im. bilja.

larasĩa, basque. [J. V.]

fejèr, veres violı, magyar, Fusz, Trivialnamen.

gizi, Malte, Delicata, Flora melitensis.

mantour, kheyley, arabe égyptien, Delule, Floræ œgypt. illustratio. 
manthoûr, kheyly, arabe égyptien, Ascherson et Schweinf.

quermezeu tchoha (= drap rouge), turc, BARBIFR DE MEYNARD, Dict. turc.

Une belle variété de cette fleur est celle qu'on appelle giroflier de Calabre ou giroflier d'Italie, tronc de chou. - RozIER, 1793.

Une autre variété s'appelle cocardeau (1) ou giroflée cocardeau (2). Bon jardinier pour 1843.

Pour les noms français des variétés du genre Matthiola, voyez l'excellente monographie de : E. Chaté, Culture pratique des giroflées, Paris, s. d.

2. - Le giroflé est une nuance du pourpre en moins foncé.

Macquer, Industrie de la teinture en soie, 1763, p. 56.

3. - Les giroflées que l'on sème le vendredi saint deviennent doubles.

Ramecourt (Pas-de-Calais), c. par M. Ed. Edmont.

- Pays d'Alost (Belg.), c. par M. A. DE CocK.

Il faut semer les ginofrèes, à jeun, le jour du vendredi saint, si on veut qu'elles soient doubles.

E. Deseille, Ciuriosités de l'hist, du pays boulonnais, p. 106.

\section{IATTHIOLA ANNUA. (3) (SwEET). - LA QUARANTAINE.}

cheiranthus annuus, nomenclature de Linsé.

cheiranthus aestiva, nomencl. de LAMARck.

quarantaine $\left.{ }^{4}\right)$, f., quarantain, m., giroflée quarantaine, f., giroflè, f.,

violier d'été, m., gıroflée d'été, f., giroflée annuelle, f., français.

tige de dix semaines, f., violier à tige annuelle, m., français, Fillassıer,

1791.

giroflier, m., français, RozıER, 1793.

quarantain millionnaire, m., Côte-d'0r, Clément-Janin, Sobriquets de la

Côte-d'Or, p. 7.

cocardieu quarantain, m., Somme, LEDIEU.

carantèno, $\int$, provençal mod., RÉGuis.

(1) Cocardieu dans la Somme, LEdieu.

(2) Pour l'historique de cette variété, dont la forme primitive a disparu presque complètement, voyez : Revue horticole, 1850, p. 17-19.

(3) Il y a deux variétés, une rouge et une blanche.

(') Ainsi appelée parce qu'elle fleurit quarante jours après avoir été semée 
goouranié de tout l'an, m., Var, Hanry, Catal. des pl. du Var. garanié de tout l'an, m., provenç. mod., Mistral, Tresor. girarde, f., giroflée blanche, f., Allier, c. par II. E. OLIvier. viooulié, m., viooulié blan, m., vioouliè d'estiéou, m., provens. mod., Mistral, Tres.

bioulèy, m., Libourne (Gironde), c. par M. Duraxd-Dégraige. bieillé, m., La Roquebrou (Cantal), c. par M. J. MALvezin. violacciocca quarantina, italien. vioule quarantin, piémontais, Colla. vieula palôvana, Plaisance, Braccifort, Flora piacentina. vioèula bianca, vioèula zinquanténna, Parme, Malaspina, Vocub. parm. viola bianca, romagnol, MoRri. aleli blanco, aleli encarnado, esp., Colmejro. milioner, Luxembourg, J. WEBER. vigeletten, Prusse, Treichel, Volksth. aus d. Pflanzenwelt. ten weeks stock $\left.{ }^{1}{ }^{1}\right)$ (= giroflée de dix semaines), anglais. gilóffer, Somer'setshire, Friend, Flower-Lore.

\section{IATTIIOLA BISANNUA.}

cheiranthus bisannuus, d'après certaines nomenclatures. yiroflée empereur, fransais, Revue liorticole, 1850, p. 19. - Catalogue de Vilmorin, 1894.

giroflée quarantaine cocardeau, f., giroflée impériale, f., français, MaIL. quarantaine royale, français, Fillassier, 1791.

\section{matThiola glabrata. (De Candolle).}

violer blanch, catalan, Costa, Flora.

aleli griego, aleli liso, espagn.. Colmeiro.

\section{MATTHIOLA TRIGUSPIDATA. (R. BrowN).}

berbesa, Sardaigne du Nord, MoRIs, Fl. sard. erva cravina, sarde logodourien, IDEM.

bacu marinu, Porto Torres (Sardaigne), IDEM.

(1) Est ainsi appelée parce que, avec certaines précautions, elle peut fleurir pændant 70 jours de suite. 


\section{IIATTHIOLA SINUATA. (R. BROWN).}

giroflee des dunes, f., Charente-Inférieure, comm. par M. E. LEMarié. violetta de mare, Alghero (Sardaigne), MoRis, fll. sard. válacu di mari, sicilien, Biasca, Flora d'Avola.

\section{mathiola VARIA. (De Gandolle).}

violur salvedi, Frioul, PIR0Na, Vocab. friulano.

\section{MAT'THIOLA GRAEGA. (De Gandolle).}

quarantaine grecque, f., français, Fillassier, 1791.

giroflée grecque, français, Le bon jardinier pour l'an XIII.

goouranié quaranten, m., Var, HaNry, Catal.

\section{MATTHIOLA FENESTRALIS. (R. BROWN).}

giroflée des fenêtres, f., français.

fenestrelle, f., giroflée chou, f., matthiole chiffonnèe, f., violier des fenêtres, m., français, Vilmorin, Fleurs de pleine terre. [ED. EDM.]. cocareau, m., Reims, Saubixet, Vocab. rémois.

viooulié de fenestro, prov. mod., Mistral.

violacciocca spennachiata, italien.

viola stražona, romagnol, MoRRI.

vioèula crespa, Parme, Malaspina, Vocab. parmiy.

baico, Gênes, Casaccia, Diz. genovese.

\section{MTTTHIOLA LIVIDA. (DE CANDOLLe).}

guelguelîn, arabe algérien, Duverrier, Les Touaregs.

tamadé, berbère, IDEM.

MatTHiola oxyGeras. (De Gandolle).

hârra, arabe algérien, DUVEYRIER, Les Touaregs.

tânehfait, berbère temâhaq, IDEM.

\section{FARSETIA AEGYPTIACA. (TURRA).}

garbä (selon FoRSKAL), haifal, melekiyeh, mourreyr, arabe égyptien, ASCHERSON et SCHWEINFLRTH. 


\section{CHEIRANTHUS (') CIIEIRI. (LINNÉ). -}

\section{LA GIROFLÉE JAUNE.}

\section{1. - NOMS DE LA PLANTE :}

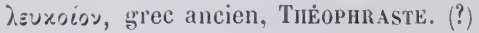

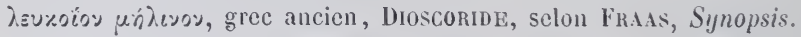

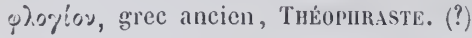

q⿳亠丷. 火itolys, grec moderne, Fraas, Synopsis.

viola lutea, latin, PLINE, XXI, 6.

violaria (la plante), viola (la fleur), anc. nomenclature, Mowat, Alphita. viola citrina, viola de romania, cheiri, alcheiri, nomenclat. du XV'os.,

DE Bosco, Luminare majus, 1496, fo 60, verso.

herba gallica, anc. nomenclat., Duchesse, De stirpibus, 1544.

leucoium luteum, leucoium aureum, flos cheiri, anc. nomenclat,

Bauins, Pinax, 1671.

galiophilée, f., anc. franc., Du Cavge, Glossarium, s. vo gariofilum.

gniroflé jausne, f., anc. franç., J. Canus, Livre t'heures.

giroflee $\left({ }^{2}\right)$ f., giroflée jaune, f., giroflée de muraille, f., français.

giroflier m., violier ${ }^{(3)}$, m., violier juune, m., français anc. et mod. (Ces

noms qui désignent le plus ordinairement la plante entière, arec ou

sans fleur, sont peu usités aujourd'hui.)

violier de murailles, m., anc. fr., Cotgrave, French dict., 1650.

géroflée jaune, f., franģais du WVII" siècle, Nouvelles instruct. pour les

confitures, 1732, p. 507; LIGER, Le jardinier fleuriste, 1718. t. I, p. 109.

girouflèye, f., Landes, comm. par M. J. DE LAP0RTERIE.

gironflee, f., Pays messin, rec. pers. - Vosges, Ilalldant, Fl. pop.

gironflèye, Vosges, Haillant, Fl. pop.

girofiée, f., Reims, Saubinet, Vocab. rémois.

gérofiée, f., Luxemb. Wallon, Dasxor.

ajunofrèy', f., Chiny (Belgique), J. Feller.

(1) Ce mot a été créé par Livé́. Il l'a tiré du nom de la plante en arabe " chciri » auquel il a

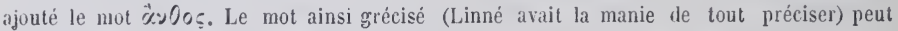
signifier fleur de main ou en main; en effet les siliques ont un peu l'air de loigts.

(2) Cette plinte est ainsi appelée parce que son odeur rappelle celle du Clou de girofle. L'Cillet porte des noms analogues pour la même raisun.

(3) Ainsi nommée à cause de l'odeur suave de violette qu'elle exhale, surtout le soir. 
ginofrée, f., Picardie. - Lorraine.

genofrèe, f., Valenciennes, HÉCART, Dict. rouchi. - Cambrésis, rec. pers.

- Luxembourg wallon, DAsNor, Dict. wallon.

janofri, m., Pays messin, rec. pers.

jalofrine, f., wallon du XVe siècle, Chronique de Jean de Stavelot. [J. C.]. jonofré, m., envir. d'Etale (Luxembourg belge), rec. per's.

gèrofle, f., Poncin (גin), rec. pers.

jalofrenne à prusain, f., spa, LEzAdck, Dict. des noms de plantes.

ginouflac, m. Lieutarles (Cantal rec. personn.

ginouflatj, m., Bozouls (Aveyron) rec. pers.

girouflada, viouliè, Basse-Auvergne, MaLral.

ginouflarlo, f., sud-ouest du Languedor, Duboul. - Hérault, Barthès. prov. morl, RÉGuss.

xinouflarlo, f., Castres, CorzıNı́, Dict. patois.

dzerofllaie, f., Su sse romande, Catal. des plantes du Pays de Vaud, 1836. dzerothayà, f. (avec th anglais doux), Bas Valais, Gultiérox, Patois de

Vionna:.

girofiasse, f., l'onts de Cé Maine-et-Loire), rec. pers.

clabéri, m., Vic-Bilh (Béarn, Lespy, Dict. béarnais.

claonéris, m., Luchon (Pyrénées), J. SAcaze.

garanié, m., garranié, m., Aix-ell-l'rovence, Borer de Fonscolonbe,

Calendrier. - Ipt (Vaucluse), Coligrox.

garanie fé, m., garranié fé, m., Bouches-du-Rhône, Villeneuve, Stal.

garanié jaouné, m., garranié jaouné. m., envir. d'Aix, GARIDEL. - Apt

(Vauclusej, Colignon, Fl. - Var, Asic.

yoouranié jaouné, m., Var, HANRs, Calal.

viole jaune, f., anc. franc., Boucherie (d'après un docum. de 1556),

Addit. au dict. de Littré.

violelte jaune, f., anc. franc., Duchesse, De stirpibus, 1541; DARI0T, De la goutle, 1589, p. 44.

violelle, f., anc. franç., Evon. Philiatre, Tresor des remedes, 1555 ,

p. 52. - Guernesè, MÉTIVIER, Dicl. fr.-norm. - Arcis-sur-Aube,

L'Arcisien pour 1865 (almanach).

violelle di kinarême, f., (= v. de carême), wallon, J. Fbller.

violaria,f., languedocien du XlVe siècle, Romania, 1883, p. 101.

violer, m., Pyrénées-0rientales, Compaxyo, Hist. nat.

violai, m., Ile d'Elle (Vendée), Rerue de philol. franc., 1889, p. 124.

vioulai, m., riolai, m., Suisse romande. Bridel, Glossaire.

yolai, m., vaudois, Durusı, Schweiz. Pflanzen-Idiot.

yolie, m., Mimbaste (Landes), rec. per's.

bioulèy, Gironde, Laterrade, 1846. 
viouli, m., Vallorbes (Suisse), Valloton-Aubert, Esquisse géogr. de Vallorbes.

vieullier, m., Lyon, Molard, Le mauvais langaye.

vioulto de mai, f., Arbois (Jura), comm. par II. H. Dorbon.

viouliè, m., Les Vans (Ardèche) rec. personn.

vioulié jaouné, m., provenç. mod., RÉGuis.

viouiè jaouné, m., Montpellier, LoRet, Flore.

jaune violet, m., Épinal. Gerbépal (Vosges), Halluant, Fl. des V.

jaune violi, m., Gérardmer (Vosges), IDEM. - Ileuse, Labourasse, Gloss. bioulié, m., Tarn, Gary, Dict. pat. - Montauban, Gaterau, Descript.

bioulié jaoune, m., Montauban, Gaterau, Descr. - Hérault, Barthės. bioulie xaouné, m., Tarn-et-Gar., LAgrèze-Fossat, Fl.

bièouliè, m., bièouleto, f., Aveyron, VArssier, Dict. pat.

bigouillè, m., canton de Saint-Germain (Lot), Soulı́,, Catal.

brioule, m., Gers, c. par M. DAIgxestous.

brigouliè, m., briouliè, m., biouliè, sud-ouest du Languedoc, Duвoul.

bruileto, f., Agenais, Bladé, Poésies populaires, Il, 126.

tchuvioli, m., Verviers et Liège, J. FELLER.

tchavioli, m., Stavelot (Belgique), J. FELlER.

tchirlive, f., Huy Belgique), J. FELler.

l'ouilhe, m., moyen et bas dauphinois, Moutier.

ıиѐ gronu, m., (= muguet graineux), Le Buisson (Dordogne), rec. pers.

graniè, m., provençal moderne, RÉGuis.

mioougranié, m., Forcalquier, c. par M. E. PLacchud.

flous jaounos, f. pl., Hérault, Barthês, Gloss.

jaouneto, f., gascon, Noulet, Flore.- Sud-0uest du Languedoc, Duboul,

Plantos.

jaounelo, f., Villefranche de Lauraguais (Haute-Gar.), c. par I. P. FAGot. jaunet, m., baguette d'or, f., Haute-Marne, comm. par M. A. Daguis.

bâton d'or, m., Haute-Marne, comm. par II. A. DAGun. - Nièvre, rec. pers. rameau d'or, m., Ineuil (Cher), rec. pers. - Normandie, JoREt, Fl. pop.

- Lyonnais, CARiot, Etude des fleurs. [ED. EDM.]. - Ce nom est surtout donné à la variété cultivée à fleurs doubles.

giroflée à biton, f., (belle variété cultivée), français, BaIlly, Ianuel du jardinier, 1825, II, p. 158.

paquette, f., Provins, Des Etaxgs, Noms pop. de pl.

mârs, m., Environs de Pamproux (Deux-Sẻvres), comm. par M. B. Souché. flou de mars, f., Gers, comm. per II. J. F. BLADÉ.

saint-jean, cassecol, Anjou, Desraux, Flore.

ravenelle jaune, f., français. 
ravenelle, f., français, Bon jardinier pour 1827. - Normandie. - Champagne. - Environs de Paris.

raguenelle, f., Mayenne, comm. par un botaniste de la Mayenne.

ramenelle, f., ram'nelle, f., canton d'Anet (Eure-et-Loir), rec. pers. -

Lieuvin (Normandie), JoRet, Fl.p. - Saint-Brice (Ille-et-Vil.), rec. pers.

ramoneuse, f., Gien (Loiret), c. par М. J. P0QLET.

comignou, m., environs de Valence (Drôme), rec. pers.

baliche, Nice, Colla.

suissard, m., Lyonnais, Cariot, Etude des fleurs. [E. Ed..].

ramoneur, m., savoyarde, f., français, Vilaroris, Fleur's de pleine terre.

[Ер. Ерм.].

musc, m., Pas-de-Calais, c. par M. B. DE KerHervé.

mazière, f., Isbergues (Pas-de-Calais), c. par II. Ed. Epмoxt.

muret, m., Namur, J. Feller. - Mons, Sigart, Gloss. montois. -

Valenciennes, HÉcart, Dict. rouchi. - Env. de Cambrai, rec. pers.

- Environ d'Hesdin (Pas-de-Calais), comm. par M. Ed. Ebsoxt.

meuret, m., Spa, LezAack, Dict. des noms wallons. - montois, SigarT,

Gil. mont.

murallier, m., env. de Boulogne (Pas-de-Gal.). c. par M. B. DE KERHERtÉ.

- wallon, FELLer,

muralli, m., wallon. SEMERTIER.

murailliez, Spa, LezaAck, Dict.

murelle, f., Saint-Iol (Pas-de-Calais), c. par II. Ev. EDMoxt.

multier, m., Warloy-Baillon (Somme), c. par M. H. CARxos.

carnache ( ${ }^{1}$, f., Condé (Nord), HĖCart.

cuiderelle, f., kéris, kiris, Valenciennes, HÉcart.

carafeee, $\left({ }^{2}\right)$ f., Haute-Marne, c. par M.A. Dagcix. - Langres, Mlcuson, Voc.

langrois. - Centre de la France, Boreav, Flore du centre. - Côte-d'Or,

ROYER, flore.

carafé, m., Allier, e. par M. C. Bourgougnon, et M. E. OLivier. - Issoire

(Puy-de-Dôme), c. par feu J. Bareire.

caraffe, f., (la variété à fieurs doubles), Verdun (Meuse), DoIsr, Hist.nat.

de la Meuse.

(1) Carnache signifie proprement crevasse de mur. La giroflée croît habituellement sur les vieux murs.

(2) Il s'agit probablement d'une variété obtenue par la culture. "A force de culture el de soins on est parvenu à avoir des fleurs panachées en brun et on les appelle giroflées caraffées. - Rozier, 1793. "- La giroflée caraffée double sappelle baton d'or. 
jalousies, f. pl., centre de la France, Boreav, Fl.; Jaubert, Gloss. fleur de parterre, f., dans quelques provinces, Rozier, 1793. erbo de vierd:s, f., Eymoutiers (Haute-Vienne), rec. pers. flour de Noustro-Damo, f., languedocien, c. par M. H. FAU. saint-Geourges, Villers-le-Sec (Haute-Saône), rec. pers. - Marnay (Haute-

Saìne), rec. pers.

violette de saint Georyes, anc. franc. comtois de Montbéliard, que nons ne connaissons que par le passage suivant en latin : "Sancti Georgii violas Montbelgardi vocant leucoium luteum. » Bauins, De plantis, 1591. yarofano, italien, J. Vicron, Tesoro, 1609, garufala, Bologne, Tosi, 1850. yaroffo, Valle del Taro, Eminanele, Valle del Taro, 1886, p. 247. violetta yialla, italien, Lexicon italico-latinum, 1648, p. 107. viola zala, Venise, BoErio.

leucojo, leucodio, leucojo yiallo, vivole a ciocche, bastone d'oro (variété cultivée), ital., TARGioNi, viola, viola gialla, violacciocca, violacciocco, italien. violàr, Frioul, Pirona, Vocab. friul. violè, Piémont, Zalli, Diz. piem.

riulè, piémontais, Colla. viör, Voghera (Piémont), Colla. riolé, Saluces, Eand, Statistica di Saluz̃zo. viole zale, pl.. Trévise, Saccardo, Flora treviy. vieula giâda, Plaisance, Braccifonti, Fl. piac. vicele zalde, Brescia, ZERSI. viola groya, sarde, Porru, 1866. baichi gianni, Gênes, DE Notaris. fior da pasqua, Vérone, Pollivi, flora veron. baichella, f., Gènes, Casaccia. Diz. genovese. balachi, Messine, P. Castelli, Horlus messanensis, 1640. barcu giarnu, sicilien, Mortillaro. válacu, Sicile, Braxca, Flora d'Avola. sirieugua, Saluces, Eand, Statist. violer groch, catalan, Costa, Flora; Cuxi, Flora. violer grog, Baléares, MarĖs, Catal. violèr groc, Minorque, Rodriguez, $1865-1868$. violetta amarilha, espagn., Dodonaeus, Herbarius, 1608. alhaili, (de l'arabe al-khîri), alheli, aleli, espagnol, Dozr, Gloss. des mots dérivés de l'arabe.

aleli amarillo, aleli pajizo, espagnol, Colmeiro, Dicc. girofe, espagnol, Missuev, Dictionary, 1623. 
aleluyes, (f. pl. ?), asturien, Rato.

viola, aragonais, Borao, 185\%. - navarrais, Gasaviella, 1880.

goiveiro amarello, portugais, Brotero, Flora lus.

aleli, galicien, Valladares.

flore de vióra, vióra galbena, micshunea, micsandra, micshunica ruginita, roumain, Brandza, Limba bot.

vasdóaga, shiboiu, roumain, Сгнас, Dict. daco-rom.

handblume, gelbes veilchen, goldlack, allemand.

veilstock (la plante) viol, veil, (la fleur), allem., Poetevin, Nouv. dict. all. bauernveigel, gelbe veigel, Augsbourg, PRITzEL ET JEss.

bohnenveielen, allemand, GRIMm, Wörterb.

kirche, Halle, Fulda, 1788 ; Martin, 1851.

stammennäyeli, Appenzell, STALDER.

näglblume, Weyersheim (Alsace, rec. personn.

strössburger, strösserli, mariastengel, vigeli, Appenzell, Pritzel.

nelke, Hesse, IDEM.

viöndli, canton des Grisons, Iden. - Berne, Stalder

mayennägeli, Schaffouse, STALDER.

mèrzfeiol, inetzfeiol, mezzfeiol, schtalfeijol, Luxembourg, J. WEBER.

metzfariule, Luxembourg, rec. personnellement.

giel foaltcher, all. de Transylvanie, Fusz, Trivialnamen.

gelber feigl, Basse-Autriche, HöғER, Wört. d. Pflanzennam. - Carinthie,

ZWANZIGER, Veræeichn.

gele viole, Brême, Brem. Wört. 1771.

winterfeigel, goldlack, Carinthie, Zwanziger, Verz.

güllake, Westplialie, Lindors, Westf. Pfl.

lämberta, (la plante a été importée en Autriche de Lombardie), lamper

Basse-Autriche, F. HöFrr.

lamberter, Transylvanie, F. HöFER.

gäl-vîlk'n, lack, Altmark, DANNEIı.

golden liken, Frise orientale, Focke, Vollisth. Pfl.

fjeellien, Brême, IDEu.

violke variété à fleur simple), loack, (variété à fleur double), Prusse,

Treichel, Volksth. aus d. Pflanzenwelt.

stockviole, Eifel, WirTgen, Vegetation d. Etfel.

maianägeli, stammanügeli, veiali, gäli, bruni veieli, poperli, poper,

poperbluamï, canton de Saint-Gall, Wartmann, Volksbot.

steenviolieren, geel violieren, flamand, Dodonaeus, Ilerbarius, 1608.

steenviolier, muurvlier, hollandais, VAN DALE. [A. DE C.].

muurviolet, hollandais, KraMers. [A. DE C.]. 
muurbloem, hollandais, flamand.

groffelier, stoffelier, stolfelier, stockviolier, violier, giroffel, flamand, DE Bo, Westvl. Idiot. [A. DE C.].

stoffelienen, flamand du Pays d'Alost, comm. par M. A. DE Cock.

banwyrt, (') anglo-saxon, Cocksrne. Leechdoms, etc. 1866.

gilly, geraflour, jeroffleris, july flower ${ }^{(2)}$, wall-july-flower, wall-

gilliflower, winter gilliflower, yellow gilliflower, yellow stock, blood wall (la variété double), wild chier, chare, wild cheir, bleeding heart, bee-flower, dans les différents dialectes de l'Angleterre, IRITTEN, Plant-names.

cherisaunce, anc. anglais, Prion, Names of brit. plants.

wallflower, gilliflower, anglais.

churl, gillofer, Shropshire, JAckson, Worlbook.

bloody warrior, Devonshire, Friend, Glossary. - Shropshire, JaCkson,

Wordb. - West Somerset, ElwoRthy, Word-book.

jelly-flower, Devonshire, FrIEND, Glossary.

giloffer, jilaffer, North Devon, Friend, Flower-Lore.

yilliver, Lancashire, Englısh Dialect Society, 188, p. 143.

walfair, anc. anglais, Mowat, Alphita.

ten week stock, (giroflée de dix semaines), anglais, Le bon jardinier, almanach pour l'an XIII.

lus-leath-an-tsamhraidh, (= half the summer plant), irlandais, 0'ReILly.

[H. G.].

bloden gorphenaf $[=$ fleur de juillet, traduction de l'anglais julyflower

qui est formé par fausse étymologic populaire sur gilliflower. La giroflée jaune est une fleur du printemps et non de l'été), gallois, CAmeron. [H. G.].

melyn y gauaf, (= jaune de l'hiver), murwyll, (= le vagabond du mur),

llys y llaw (= herbe de la main), gallois, Hugh Davies. [H. G ].

genofl, breton, Nomenclator; P. GRÉGoIRE. [E. E.]

genoflès, vyoletès melen, breton, P. Grégoire. [E. E.]

violet melen, breton, Nomenclator. [E. E.]

gilouflee, girauflé, breton de Vannes, DE l'A**, Dict. [E. E.]

givézen c’hénofl, Finıstère, Crouan.

jénoflez, breton, LIÉGARD.

lakkviol, suédois.

gyldenlak, danois et norwégien.

(1) A derivation of bana, a manslayer, from the bloodstained colour of petals. - CoCKAYNE.

(2) Corruption de gilliflower; la plante fleurit en mars et non en juillet. 
gule fioliter, danois, JENSSEN-Tusch.

¿̇luta fijala, tchèque, A. Müller, Synonym. Namen, 1866.

macica fiolkowa, polonais. Nemnich, Polygl. Lexicon.

gvózditchnaja fialka (= violette clou de girofle), russe.

šebenik, żuti šeboj, żuta ljubica, żuta viola, vihojla, ¿̇uta vihojla, ¿̇uta rumanija, spanska fiolca, rmene fiolce, serbo-croate, Šulek, Jug. im. bilja.

sárga viola, magyar, Fusz, Trivialnamen.

khîrî, kheiri, arabe. Le mot kheiri est d'origine persane.

khiri, kherounfla, arabe algérien, Florian Pharaon, Voc. ar.

khili, arabe de Constantine, Cherbonneau (dans Journ. usiat., 1849, p. 66. khaili, arabe marocain, DoмBAY, Grammat. mauro-arabica.

mentoûr, mantoùr asfar, oumm qourounfl, arabe syrien, BERGGRen, Guide arabe.

manthoûr, arabe égyptien, Aschersox et Schweinfurth.

menthour, arabe, IвN-BEїтнAR.

mantsour, arabe, Leclerc, Révélat. des énigmes, 1874.

o'ssaifira, arabe, IbN-BeïthaR (Edit. Leclerc), II, 68 et $\mathbf{4 5 1 .}$

chèbbouy, frenk mènèlichéci, turc, MaLlour, Dict.

chèhbouy, frenk benefchè, frenk menechè, turc, Ciнаc, Dict. daco-rom.

firenk benefchèsi, turc, BARBIER DE MEYNARD, Dict. tur'c.

shahprak, shahpourak, garin dzaylik (= fleur d'agneau), arménien, ALishan. [ER. L.]

Pour les noms français des variétés cultivées de la giroflée voyez : E. Chaté, Culture pratique des giroflées, Paris, in-18. s. d., 95 pages.

2. Es fresco coumo uno xinouflado. Elle est fraîche comme une giroflée; se dit d'une jeune fille.)

Castres, Couzıxie, Dict. patois, 1847.

3. Viola zala, amôur cala - Viola gialla, amor che scema. Diciamo in proverb. E gli amanti si tengono dal dono scambievole delle viole gialle, per tener lontano l' adagio.

Bologne, Coronedi-Berti, Voc. bologn.

Dar et viol - Licenziare l'amoroso, e per similit : Cacciare checchessia. I contadini si servono appunto delle viole gialle per licenziare l'amante.

Bologne, Coronedi-Berti, Vocab. bologn.

4. Pour avoir des giroflées doubles il faut les semer le vendredi saint (Seine-et-0ise; Deux-Sèvres), avant le lever du soleil (Loiret), à jeun (Touraine). 
5. Les femmes d'Escoussens et de Labraguière (Montagne noire, Cévennes) ont l'habitude, le jeudi saint, de mettre dans leur poche des graines de violier mêlées avec de la terre et, durant le chant du Stabat, elles agitent vivement ce mélange; elles sont convaincues que ce moyen leur procurera des fleurs doubles.

A. DE Chesnel, Usages de la Montagne noire, 1839.

\section{BARBAREA (GENRE). (R. BROWN). - L'HERBE DF. SAINTE-BARBE.}

\section{1. - NOMS :}

barbarea, latin du moyen âge, Diefenbach, Glossar. med. lal.

nasturtium hibermum, carpentaria, herba sancta, sanctie Barbarae herba, anc. nomenclat., J. BAcHix, De plantis a sanctis nomen habentibus, 1591.

eruca lutea latifolia, sinapi anreste, scopa regia, sideritis latissima, pseudobunias, carpentorum herba, eruca palustris, bunium adulterinum, anc. nomenclat., Bauhin, Pinar, 1671.

erysimum barbarca, nomenclat. de LINxÉ.

herbe sainte Barbe, f., anc. fr., J. Baunin, De Plantis, etc., 1591.

barbarée. julienne jaune, vélar barbarée, vélar de Samte-Barbe, girarde jaune, français.

herbe de sainte Barbe, f., herbe aur charpentiers, f., français.

herbe i sainte Barbe, f., herbe yrasse, f., aourrie, f., cresson, m., Aube, Des Etangs, Noms des $p l$.

herbe de sainte Maryuerite, f., herbe de saint Julien, f., Anjou, Dssvacx, Flore.

yèp' du sint' Bäb', f., yèp du sint' Bôr, f., wallon, J. Feller. herbe de saint-Syluain, f., Centre de la France, Jaubert, Gloss. herbe Notre Dame, f., Charente-Inférieure, comm. par M. E. LemariÉ. erbo de santo Barbaro, f., Var, Amc., Consilèr. sur Brignoles. - Apt, Colignon, Catal. - Gard, c. par H. P. FesQlet.

cresson de terre, m., cresson vivace, m., français, L. Dubors, Pratique du jarlinaye, 1821, p. 77.

cresson de jardin, m., cressonnelte de jardin, f., Anjou, Desvaux.

cresson des vignes, m., Mayenne, c. par un botaniste de la Mayenne. -

Anjou, Desvaux, Fl. de l'Anj.

cresson américain, m., Haute-Marne, c. par M. A. Daguin.

rondotte, f., français. - Côte-d'Or, Rorer, Flore. - Haute-Marne, c.

par M. A. Daguin.

roundoto, f., Apt (Vaucluse), Colignon, Catal. 
rondelotte, f., rond lotte, f., Bainville-aux-Saules (Vosges), Haillant, Flore pop. - Haute-Marne, c. par M. A. DaGuin. rondette, f., rondelette, f., Haute-Marne, c. par M. A. DAGUin. herbe à la brunette, f., Centre de la France, JAUBERT, Gloss. cassoule to jaouno, f., Toulouse, Tournon, Flore. roquette des jardins, f., français, LiTTRÉ, Dict. tiran (') m., Brive (Corrèze), c. par M. G. DE LÉPINAY. gras capou, Sud-0uest du Languedoc, DuBoul, Plantos. herbe grasse, f., Treigny (Yonne), c. par M. A. de GuErchy. aragone, f., Haute-Marne, c. par M. A. DAGuin.

aragonne gonne, f., (= julienne jaune), Haut-Boulonnais, c. par M. B. DE KERHERVÉ.

pied de vache, m., Forez, A. LeGrand.

barbarea, ruccola palustre, ital., Ambrosini, Phytologia, 1666.

erba barbara, erba santa Barbera, italien, TARGIONI, Diz. bot. erba santa Barbora, milanais, BANFi, Voc. mil.

erba de santa Barbôra, Plaisance, Bracciforti, Flora.

erba d'santa Barbra, piémontais, Capello, Dict. piémontais.

barbora, Tessin, DuRHEm.

uruga sarvaggia, sicilien, LAGusi, Erbuario, 1742.

gradisèlla, raviscetta, milanais, CHERUBINI.

ravisc selvarlegh, Lodi, Pavie, Cherubini.

yerva de santa Barbara, espagn., Bauhin, De plantis, etc., 1591.

yerba de santa Barbara, esp., Colveiro, Dicc.

herva de santa Barbara, port., Brotero.

erba de santa Barbara,f., galicien, Valladares.

berillo, Canaries, WEBB, Hist. nat.des Canaries.

barbushóra, roumain, BRANDzA, Limba botan.

cruzatcé, roumain, Ciнас, Dict. daco-rom.

krutzaitzie, roumain de Transylvanie, Fusz, Trivialnam.

winterkress, barbellkraut, anc. all., DIEFENBACH, Glossar.

winter kräss, all., BAuнiN, De plantis, etc., 1591.

barbenkraut, barbenhederich, allemand.

saurer hederich, Eifel, Wirtgen, Vegetation.

sauersinef, m., Luxembourg, J. WEBER.

racketenkraut, Suisse allemande, Durheim, Idiot.

rapunzel, Prusse, Frischbikir, Preuss. Wört. (1) Cette plante a la réputation de faire suppurer les plaies, mais elle les fait creuser,
dit-on. - Communic. de M. G. DE LÉPINAY. 
Barbarakraut, rekliaut, Carinthie, ZWANzigER, Ver: schnödsenf, habichtskraut, Silésie, Pritzel.

winterkersse, steenkruydt paree que cette herbe guérit la gravelle), anc. flamand, Dodonaeus, 16.14. [A. DE C.].

barbarakruid, winterker's, néerlandais. [A. DE C.]. winter-kers, flamand, DE GORTER.

winter cress, anglais.

american cress, belleisle cress, bank cress, french cress, land cress, cassabully, dialectes angl., Britten.

treabhach, gaélique écossais, CAMerov. [H. G.l.

berwer y gauaf (= cresson d'hiver), gallois, HuGH DAvies. ]H. G.]

costog y dom (= chien du fumier), gallois, J. DAvies, 1632. [H. G.]

berwerfrainc (= cresson français), gallois, Meddygon Mylllfai. [H. G.]

louะaouen santez Barba, breton, LiÉGARd ; Crovas.

lousaoën santès Barba, breton, P. GRÉGoIRE. [E. E.]

Barbaras ïrt, vinterlirasse, suédois.

vinterkarse, danois.

kårsticirel, norvégien, JENSSEx-Tusch.

repnica, ritnja, svete Barbare zele, suete Barbare rośa, serbo-croate, SULEK.

barbara, barbora, swiẹtej Barbary siele, polonais, LINDE.

téli tormants, magyar, Fusz, Trivialn.

bübara, arabe syrien, Berggrex, Guide.

2. USAGES :

Les feuilles pilées sont souvent employées pour les meurtrissures. On en mange les jeunes pousses en salade.

\section{TURIRITIS GLABRA. (LINNÉ).}

turritis, turrita, vaccaria, anc. nomencl., Bauhis, Pinax, 1671.

tourette, f., français, Neмsich, 1793; Bon jardinier pour 1827.

tourelle, f., français, Nennich, 1793.

turicel, roumain, BRANDzA, Limba bot.

tower-mustard, anglais, PRIOR, Names.

thurnkraut, anc. all., DIEFENBACH, Glossar.

thurnsenf, thurnkohl, thurnsaat, bergkresse, waldkohl, allem., NЕммicн, Lexicon.

torenkruid, hollandais.

turren-kruid, flamand, DE GoRTER. 
twriged, gallois, Hugir DAvies. [H. G.].

tiirnört, räkkentraf, suédois dialect., JENSSEN-Tusch.

türnsinnep, jomfruérter, norvégien, IDEM.

vild kïl, tïrnurt, danois, IDE.r.

plemenita vlas, svilene kose, serbo-croate, ŠulEk.

\section{ARABIS (Genre). (Linvé). - L'ARABETTE.}

arabette, f., français, FiLl.Issien, 1791.

gascaritca, roumain, CuHAc, Dict; Brasdza, Limba bot.

günsekraut, allemand.

honigschub, hollandais, NEMnICH, Lexicon.

scheeflelk, néerlandais. [A. DE C.].

wall-cress, anglais.

alyssum, anise, sweet Alice, Devonshire, Frimid, 1886, p. 487.

snow on the mountain, snow in summer, milk and water, Sussex, Friend,

Flower-Lore, 1886, p. 487.

berwr y faywyr (= cresson du mur), berwor y cerriy $=$ cresson $\mathrm{du}$ rocher), gallois, Hugh DAries. [H. G.]

gisemad, armkial, danois, JENSSEN-Tesch.

vårel, norvégien, IDEM.

gesiowtia, polonais.

husemit, tchèque.

repnjak, scrbo-croate, ŠLLEK.

lenok polevyi (= petit lin champêtre), petit russien, Schmaliauses. [Th. V.]

\section{ARABIS ALPINA. (LINNÉ)}

corbeille d'argent, f. français, Viluoriv.

arabette des Alpes, f., giroflier des Alpes, m., français, Nemxici, Lex. hungerblümchen, allem., A. HëLLER, Synonym. Namen.

hladovenka, tchèque, A. MüLLER.

lady's cushion, bishop's wig, dusty husband, dialectes anglais, Britten, Plant-names.

ARABIS ARENOSA. (Scopoli.)

cholot, m., Aube, Des Etangs, Noms des pl. 


\section{ARABIS HIRSUTA. (Scopoli).}

turritis hirsuta, nomencl. de LINNÉ.

crasson d'âw, m. (= cresson d'oie), wallon, J. FelLer.

baccellina, erba baccellina, ital., TARGIONI.

rasparoèula, Parme, Malaspina, Voc. parm.

torrecruydt, anc. flamand, Dodonaeus, 1644. [A. DE C.]

\section{ARABIS THALIANA. (LINNÉ).}

arabette des dames, français, LoISEleur, 1828.

arabette rameuse, f., français, Кемлісн.

famine, f., Anjou, Desraux, Flore.

skrén-blom, Norvège.

mûl, Danemark.

\section{NASTURTIUM OFFICINALE. (R. BRow'). - LE CRESSON DE FONTAINE.}

\section{1. - NONS DE CETTE PLANTE :}

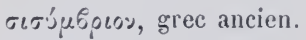

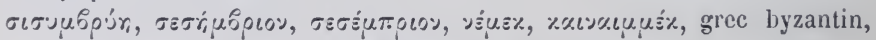
LANGKAVEL, Geschichte der Botanik.

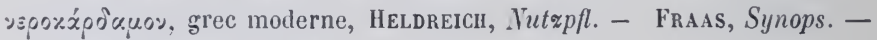

Tсhнататенғ, Asie min.

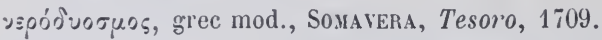

xúpơuov, grec mod., Portius, Dictionarium, 1635.

sisymbrium, latin, PLINE.

sisimbrium, lat. du Moyen-Age, Germania, 1888, p. 310 ; Zeitsch. f. d.

Alterth., 1872, p. 362.

sisimbria, lat. du Moyen-Age, Descenet.

nasturtium, nasturcium, cardamum nigrum, latin, SCRIBONILS LARGUS

( ler $^{\mathrm{e}}$ s. apr. J.-C.), Marcellus EMpiricus (IVe s. apr. J.-C.), selon

MEYER, Geschichte der Botanik.

nasturcium, lat. du Moyen-Age, Germania, 1881, p. 402 et 1888. p. 307. nasturcum, latin, Marcellus Esipiricus (IVe s. apr. J.-C.), Arch.f. latin

Lexicogr., 1886, p. 262.

crissonus, lat. du X'e siècle, LitTRÉ, Dict. s. vo cresson.

crisonium, lat. du Moyen-Age, Du CANGE, Glossar. 
cresco, lat. du Moyen-Age, EARLE, Engl. plant names.

cardamum, lat. du $4^{\mathrm{e}} \mathrm{s}$, Marcellus EMpiricus.

carlamus, lat. du Moyen-Age, Diefenbach, Gloss.; Germania, 1881, p. 40't

et 1888, p. $303 ;$ Zeitsch. f. d. Alterth., 1843, p. 370 et 1872, p. 336.

cardamonium, lat. du Moyen-Age, Germania, 1881, p. 402.

senecium, gracius, gratius, gracinus, cracius, lat. du Moyen-Age, DIEFENBACH, Gloss.

senapio, senecium, thymbra, thymbreum, Gloses latines du Moyen-Age citées par GRIMn, Wörterb.

carista, kerso, lat. du Moyen-Age, ZEuss, Gramm. celt., 1871, p. 1076.

[H. G.].

senacion, lat. du Moyen-Age, Germania, 1888, p. 309.

senationes, cressones (pluriel), Маттнаeus Silvaticus (auteur du $14^{\mathrm{e}} \mathrm{s}$ ), cité par Du CaNGE.

cressiones, crision, senescion, senacio, cardamen (la semence), lat. du

Moyen-Age, Mowat, L'Alphita.

senaciones ( ${ }^{1}$, , crescionum, nomencl. du $15^{\mathrm{c}} \mathrm{s}$., J. Camss, L'opera salernit., p. 116.

cardamus domesticus, cardamus, lat. du $15^{\mathrm{e}}$ siècle, DE Bosco, Luminare majus, 1496, fo 20 recto.

nastu:'clum aquaticum, lat. du Moyen-Age, Mowat, Alphita; J. Camus, L'opera salern.

damasonium, lat. du Moyen-Age, Germania, 1888, p. 303.

cartilayo, lat. du XIVe siècle, Escallier, Rem.

nasturtium aquaticum, sisymbrium, cardamine, vella Galeni, sium, laver,

cardaminum sion, cresso, laver odoratum, sisymbrium cardamine, sisymbrium aquaticum, sion Cratevae, senecio Serapionis, sium majus, anc. nomenclat., Baumin, Pinax, 1671.

sisymbrium nasturtium, nomenclat. de LINxÉ.

kersuns, m., anc. fr., Mowat, Alphita.

kersun, m., anc. franç., P. MEXER, Deuxième rapport s. une mission.

querson, m. anc. franç., J. Casus, Livre d'heures.

kerson, m., anc. fr. - Valenciennes. - Pas-de-Calais. - normand. -

picard. - manceau. - nivernais.

karson, m., Haut-Boulonnais, c. par M. B. DE KERHERVÉ.

kërchon, m., Tannay (Nièvre) rec. pers.

curson, m., Segré (Maine-et-Loire), rec. pers.

(1) L'auteur de l'Opera salernitana Circa Instans dit que si l'on veut désigner le Cresson aquatique le mot Senaciones doit ètre employé au pluriel. 
crissous ewages, cressouns, anc. fr., Mowat, Alphita. cressons, m., anc. franc., Escallier, Rem.

croisson, mi, anc. fr., Lagadeuc, Catholicon, 1199; Picot et Niror, Noul. rec. de furces, 1880, p. 194.

cressaien: français du $1^{\circ}$ siècle, Bibliothèque de l'Ecole des Chartes, 1869, p. 328 et p. 330.

cresson ( $\left.{ }^{1}\right)$, m., cresson d'eau, m., cresson de fontaine, m., français. crechon, m., heurson, m., krechelon, m.. Vosges, Halluaxt, li. p.

crèchon, m., Morvan, Chisbure. - Plancher-les-Mines (Haute-Saône,

Poulet. - Env. de Saint-Qnentin (Aisne), ree. pers.

crèchan, m., Chiny (Belgique), J. FeLder. - Etalle Belgique) rec. pers. cressan, m., Deux-Sèvres, c. par M. B. Souchè.

crasson, m., Malmèdy (Pays wallon), Zeligzor. - wallon, Feller.

crîzon, m.. Ponts-de-Cé (Maine-et-Loire), rec. personn.

creuzon, m., Saint-Pol (Pas-de-Calais), c. par M. Ed. Ed.ront.

crissou, m. Fournels (Lozère), ree. pers.

crisso, m., Thiers (Puy-de-Dòme), rec. pers.

crinchon, m., Coligny (Ain), Clédat, Le patois de Coligny, 1887.

crinson, m., Centre de la France, Jaubert, Gloss. - Suisse romande, Bridei, Gloss.

crescian, m., mentonais, ANDREws, Vocab.

créchou, m., Arrens (Hautes-Pyrénées), c. par M. M. CàÉl.AT.

creisson, anc. prov., Romania, 1892.

cressou, m., Argelès Hautes-Pyrènées), par M. P. TARissan.

créissoun, Bouches-du-Rh., Vilbeveure. - Var, rec. pers. - Forcalquier,

c. par M. E. Platchld.

créssoun, m., Gers, c. par II. H. Daignestous.

créissou, m., Haute-Vienne rec. pers, - Corrèze, DE LÉPINAY, Noms patois.

- Montpellier, Loret, Flore. - Haute-Loire, Arxadd, Flore. -

Livron Irôme), c. par M. E. H. Sibourg. - Ardèche, rec. pers. créichoun, m., créissou, m., Briançonnais, c. par M. Chabraxd.

crusson, m., wallon, J. Feller. - Marnay (Haute-Saône) rec. pers.

crussoun, Labouheyre Landes) c. par II. F. ARxaulis.

crussoun, m., creissillıou, m., Hérault, B.ırthès, Gloss.

crissoun dei vala, m., Toulon, Pıтоuт, Plantes médicinales.

(1) On fait venir ce mot du latin crescere, parce que le cresson de fontaine et surtout le cresson alènois ont une croissance rapide. - Cette ètymologic est invraisemblable. L'n grand nombre d'autres plantes, les champignons, particulièrement, ont une croissance rapiule; mais aucune dientre elles ne porte le nom de cresson. 
créssélou, m., Lieutadès Cantal . rec. pers. - La Roquebrou (Cantal), comm. par M. J. Maliezin. - Tauriac (Lot, ree. pers.

créssélous, m. pl., Castelnau de Montmiral (Tarn.), rec. pers. (Le mot ne s'emploie qu'au pluriel).

créissélou, m., Tarn, GART, Dict. patois. - Sud-Ouest du Languedoc, Deboll, Plantos. - Lot, Soulié, C'atal.

crécliélou, m., Aurillac (Cantal, rec. personn. - Le Buisson (Dordogne), rec. personn.

créichelou, m., Brive (Corrèze), DE LÉPINAY, Noms pat.

crichilhou, m., env. de Careassonne, LAfFage.

gréchéroun, m., Libourne (Gironde), c. par II. L. DuRand-DÉgrange. Marensin (Landes) e. par M. l'abbé V. Forx.

crécheiroun, m., Gironde, LATERRADE.

creysselou negre, m., Aveyron, Visssien, Dict.

graïssélou, Sud-0uest du Languedoe, Dtboul, Plantos. - gascon, Novlet,

Flore. - Tarn-et-Gar., LAgrìzE-Fossat, Flore. - Tarn, Lot, comm.

par II. J. DATMARD.

graïssou, m., Gard, c. par M. P. Fesquet.

gréissou, m., canton de Laurières (Haute-Yienne, rec. pers.

greissoun. m., Gard, Pouzolz, Flore du Gard.

gréssonn, env. de Barbaste Lot-et-Garonne), comm. par M. l'abbé

L. DARDY.

grachon, m., Combloux Haute-Savoie, rec. pers.

gresson, Naintré (Vienne), rec. personn.

greirous, m. pl., Oloron, LEspi, Dictionn. béarnais.

grechous, ni. pl., béarnais, IDEм.

grécliou, m., Eymoutiers Haute-lienne), rec. personn.

gracilloun, m., Luchon (Pýrérées). J. SAcAzE.

grécillioun, m., Dunes (Tarı-et-Gar.), rec. pers. - Gers, e. par M. J. F.

BLAdÉ.

kenneron, m , kenn'con. m., Mayenne, c. par un botaniste de la Mayenne.

- Valenciennes, Hécart.

crenesson, m., Gâtinais (Orléanais), c. par M. L. Malox. - Env. de Blois,

THibaUlt.

knesson, m., Anjou, Desvali, Flore.

senacions, m. pl., senessons, m. pl., français du $15^{\mathrm{e}} \mathrm{s.}, \mathrm{J}$. Canus , L'opera. senasson, ni., ane. fr., Duez, Dict. franc. ital., 1678.

cresson de caillay, m., anc. franç., C. STEPhaxus, Arbustum etc.. 1538, p. 30 .

cresson de cailler, m., fr. du $16^{\mathrm{e}} \mathrm{s}$, La Laison rustique.

caili, environs de Rouen, Le prévoyant jardinier pour 1781. 
cailli (1), Rouen, IIme Gacon, Dictionn. rural, 1808.

berne $\left({ }^{2}\right)$, f., Aunis, L. E. MEYER. - Charente-Inf., comm. par M. E. Lemarié. biône, f., Montbéliard, Contejean, Glossaire.

berle, f., crasson, m., Haute-Bretagne, P. SÉBılot, Trad. et Sup. de la Haute-Bretagne, II, 334.

carson, Ferrare, Nannini, Vocab. ferrarese. - Parme, Malaspina, Vocab.

- Plaisance, Bracciforti, Flora. - lombard, Pollini, Flora veronensis.

chërsun. chërsunil, piémontais, CoLla.

crsson, Mont-Cenis, Re, Flora segus. - Saluces, Eand, Statistica. cresson, env, de Turin, Re.

crasson, Piémont, Ann. de l'observatoire de Turin, 1811, p. 105. - lombard, Pollini, Flora veronensis.

crescione, italien, RogerI, Cataloyo d. piante, 1677. - napolitain, Mussafia,

Ein alt neapolit. reg. sanit., 1884, p. 30.

criscione, Barbaggio (Corse), rec. pers. - napolitain, Gusumpaur.

crisciuni, sicilien, Cupani, Hortus catholicus, 1696.

crisciuni, crisciuneddu, sicilien, Buxca, Flora d'. Ivolu.

erba crescione, env. de Pérouse, Zanetti, p. 28.

nastruzzo, Padoue, Patriarchi.

nastursio, nastursi, piémontais, Colla.

mastruะzo aquatico, grasson, gresson, vénitien, BoErio, Diz.

mastrozzu, sicilien, Mortullaro.

masturço, anc. napolitain, Mussafia, Ein alt neapolit. rey. sanit., 1884, p. 30 .

listör'c, piémontais, Zall, Diz. piem.

grassón, Mantoue, Cherubini. - Vérone, Pollini. - Milanais, BıNFi.

yressa, Vérone, Pollini. Flor'a veronensis.

yrassù, Brescia, Melchiori, Vocab. bresciano ; Zersi.

agretto, italien, Axgulllara, Semplici, 1561; A. Petronius, De victu roman., 1581 , p. 90 .

(1) Selon $\mathrm{M}^{\text {me }}$ Gacon, on cultivait àtrefois en grand une excellente qualité de cresson à Cailly, près Rouen; de lá vient que cette plante était connue à Rouen sous le nom de Cailli.

On lit dans le Recueil de poesies françoises, t. I, 1855, p. 94 :

Avec du cresson de Cailly

Et puis quelques herbettes fades

Feray cent sortes de salades

Pour réjouyr les compagnens.

(2) On appelle ainsi le cresson quand il est fructifié et qu'il n'est plus bon pour la salide. Il ressemble alors à la berle. Comm. par E. L.enarié. 
allegretto, environs de Rome, A. Petronius, De victu romanorum, 1581, p. 90.

cicembro, italien, TARgion, Diz. bot.

cardamo, Pouille, Avguillara, Semplici, 1561.

cresson, grasson, frisso, sgrigulo di aghe, rucule di aghe, nastrizz, Frioul, Pirona.

martu̇zи, sarde méridional, SPano.

martuizzu de arriu, Sard. du Sud, Moris, Flora.

nascienzo, napolitain, Mussafia, Ein alt neapolit. Reg. sanit., 1884, p. 30. acione, aschione, Alghero (Sard.), Monis, Flora.

ascione, sarde logodourien, SPANo.

ascioni, sarde septentrional, SPANo.

cannéle, livrije, aschióne, pisciacchiari, Alruzzes, Fivanore, Bot. pop.

sanaccione, Tarente, Mussafia, Ein altneapolit. reg. sanit., 1884, p. 30. mastuerzo acuático, esp., Colmeiro, Dicc.

berro, esp. du $15^{\circ}$ s., Dozy, Suppl. aux dict. arabes, II, 153. - Canaries,

WEBв, Hist. nat.

agrião, portug., Brotero, Fl. lusit.

brizo, galicien, Cuverro, Dicc. gall.

berro, galicien, Valladares.

crexec, creixens, catalan, Costa, Flora.

crexens, ancien catalan, Agustin, Secretos de agriculturu, 16:16; cat. mod. cardama, roumain, BRANDZA.

sisimbrima, anc. haut all., Zeitsch f. 九. Alterth., 1872, p. 362.

burncrasse, anc. liaut all., DesceMeT.

cresso, m., chresso, m., chressa, f., anc. haut. all., Diefenвach.

brunnekresse, bornkresse, moyen haut all.

kressig, brunnenkresse, wasserkresse, bachkresse, allemand.

kres, kressech, allem. des Alpes vénit, SchuELler.

karsch, Aix-la-Chapelle, Grimm.

burekascht, Luxembourg, J. WeBER.

graschum, Engadine, DurheIr.

lasse, f., Gottingue, Sснамвасн.

bornkassen, Brême, Brem. IVört. 1771.

bornkirsche, (la seconde partie du mot, kirsche, vient d'une fausse

étymol. popul.) braunkirsch, Thuringe.

burngress, Grosbliderstroff (Lorraine), rec. personn.

water-kersse, anc. flam., Dobonalus. [A. DE C.]

water-kers, flamand et lollandais. [A. DE C.].

watersalade, flamand, DE B0. [A. DE $\left.\mathrm{C}_{2}\right]$.

caerse, cyrse, cressae, anglo-saxon, Cockarne. 
watercresses, anc. angl., Mowat, Alplita.

well-grass, well-kir'se, well-yerses, écossais, Janieson, Dictionary.

water cress, waler yrass, water crushes, brown cress, dialectes anglais,

BRITTEN.

biror, anc. irlandais, W. STokes, Corrmac's ylossary. [H. G.].

bilar, moyen irlandais, W. Stokes, Anecilola oroniensia, 1890, p. XCV. [H. G.].

bilur uisgi $=$ cresson d'eau), moyen irlandais, W. Stokes, (dans Revue Cell., IX, 1. 238.

billar, bill'uyh, irlandais, J. КEоGH, 1735. [H. G.]. bior'ar, irland. mod., Jorce, Il, p. 325. H. G.].

biolair, durlus = waterplant, dobharlus (= waterplant), gaélique écossais, Caneron. H. G.].

burley, mannois, Cregeex; J. Kelly. [H. G.].

beru'r y du'r, = cresson de l'eau), mintys y divr (= menthe de l'eau, gallois, J. DAViEs, 1632. [H. G.].

berw. y /lynonu $=$ cresson des fontaines), gallois, Hugh Davies. [H. Gi.

ber'w' duff (= cresson de l'eau, gallois, J. Morgan. H. G.]. beler, ancien comique, Zerss, Gramm. cell., 187l, p. 1076. H. C.]. beler, breton, Lagunelc, Catholicon; Nomenclator; etc. [E. E.]. crẹson dour, breton, P. GRÉgonin. E. E. .

kreson, breton de Tríguier. [E. E.]. rïgsenap, wild senep, suédois, Jexssex-Tusch.

resucha, polonais, LINDE.

mitien, polonais de la Prusse, Treichel, Volksth. aus d. Pfanzenn. jéronkha, (= lo-manger des animaux), russe, Schualhausex. [Tн. V.]. jericha, rericha, rnker', lustinec, tchèque, A. Mülder, Synomym. Namen. rèricha polocinj. riericha vodnj, tchèque, Palkowitsch.

drayucac, drayusac, derezga, ljutolista derezya, drezgu, yarbac, yarboc, yarboc, yarbok. grbac, yrbaca, grbak, yrbaja, grbanj, mala yrba, ljubidrag, puscica, resinit, sludencica, lurina, uyas, vodena yorusica, gurdun, gresione, greśstu, greśtur, kres, volna lreśa, lireson, hivesun, lives, todeni lives, livesica. vodua kirèsa, vodna locika, ljuti moliris, divjı hren, vodeni ren, nastruc, sdencui bobounjak, bruntires, punkres, gorusica vorlena, serbo-croate, Šulek.

kehrshi, kehrses, letton, Stender, Lelt. lexicon. uhders schlieh'schi, letton, HurEL.

resukai, lithuanien, JAcom, Litauische I'flanzennamen.

berro, kerchun, basque. [J. V.].

halliko ker'sid, esthonien, Hupez. 
qernounech, guernounech, arabe algérien, LECLERc, Traduction d'Ibn Beïlhar, III, 67; Floman I'haraon, Vocab. arabe. yuerminech, kabyle, Hanotedu, La Kabylie. querminech, berbère, E. G. PARIS, Vingt-deux mois dans le Saluara, 1866. gerwennesh, hhurf, ziyata, arabe marocain, DomBAY. garlinous, arabe du Boghar (Algérie), Debeadx, Catalogue. horf, arabe égyptien, Alpwus, De medicinâ Aeygptiorum, 1645, p. 35. horf el $m \dot{a}$, arabe, Iв. Beithar (Traduction Leclerc, I, 430. reschâd el mî, qourrat el äin, arabe syrien, Berggren, Guide. harra = l'épicée), arabe algérien, Haxoteau, La Kabylie; E. G. l'Aris,

Vingt-deux mois dlans le Sahar'a, 1866. qreximi, sia, MaIte, Delicata, flora me'. boulaq oteu = herbe de fontaine), turc, Barbien DE Meriand, Dict. turc. sou térési, turc, Maldouf, Dict. tur'c.

djeri kotém (= cresson d'eau), djerdjeroul, arménien, IlısHax. [Er. L.]. canzia, langue inconnue du pays de Wiesbade au XIIe siècle, Descenet.

Pour I'historique du cresson voyez Av. Chatr, Note sur le cresson de fontaine (dans Bull. de la soc. botan., 1858, p. 158-167.

2. L'endroit où l'on cultive le cresson s'appelle : cressonaria, cressoneria, gressonaria, cressoria, latin du Moven-Ige, DE

CANGF.

cressonnerie, f., anc. fr., DuEz, 1664.

cressonnière, f., français.

gréssounèro, f., env. de Barbaste (Lot-et-Garonne), c. par II. l'abbé L. DARDY.

kersonnière, f., Artois, c. par M. Ed. Edmont.

3. Une courte poésie macaronique de l'an 150 s nous tat connaitre les propriétés du cresson :

Omnibus sit notum
Que cresson signifie,
Purgat stomachum
Et vuyde la vecye,
Donat appetitum
Et les reins mundifie.

Bibl. nat., ms. fr. 4.431 fo 76 . (Je dois la connaissance de ces vers á II. J. Couraye du Pakc.)

4. Net comme un ereissoun.

Locution provengale, Mistral, Trésor. 
Sain comme du cresson. m., Commerson, Un mari à l'étouffée, Vaudeville, 1854, p. 3.

5. «... L'Anglaise, toujours froide comme du cresson, semblait lieureuse de son flegme... »

Balzac, Gaudissart II. (Roman).

6. Sur la culture du cresson de fontaine daus la province d'Artois, dès le XlVe siècle, voy. Bull. de la Soc. botan. de France, V, 743.

\section{SUPFRSTITIONS.}

Le cresson ne vient que dans les ruisseaux où l'on a roui du lin; c'est la graine de lin qui le produit.

Haute-Bretagne. P. SÉBlLlot, Trad. ct Sup. de la Haute Bret. II, 33'.

Not long ago, an old woman was found, on a may morning, at a springwell cutting the tops of water cresses with a pair of scissors, muttering strange words, and the names of certain persons who had cows, also the words : " s' liomsa leath do choud sa " (= half thine is mine). She repeated these worls as often as sle cut a sprig, which personated the individual sle intended to rob of his milk and cream.

Ecosse gaelique, Cameron, p. 109. [H. G.j.

\section{N.ASTURTIUM SILIESTRE. (R. BRow). -}

\section{LA ROQUETTE SAUVAGE.}

sisymbrium sylvestre, nomencl. de Lixvé.

herbe à l'oie, f., herbe à l'empereur, f., Anjou. Desvaux.

bramefan, env. d'Aix en Pror., Garidel.

nasitort salbatje, mı, yraïsselou salbatje, m., rima, m., Sud-0uest du

Languedoc, Déboul, Plantos.

nasitort sauvage, m., français, Cotgrave, 1650.

roquette sauvage, f., français.

rouqueto fero, f., Aix, BoYer DE FonscoL., Calendrier. - Forcalquier

Basses-Alpes, c. par M. E. Plauchud.

aruga sarvaja, Schierano Piémont), Colca.

arughetta, piémontais, CAPELLO, Dict. piém.

rastielutt, rasçelutt, Frioul, PIRoNa, Vocab. friul.

oruga bra'a, portugais, Nemsich.

wasser-rauke, wilde rauke, waldrauke, allem.

waterraket, bosch-uterkers, aliker-waterkers, flamand et hollandais.

[A. DE C.]. 


\section{NATURTIUM AMPHIBIUM. (R. BROWN).}

sisymbrium amphibium, nomencl. de LinNÉ.

raphanus aquaticus, latin des pharmaciens, Mérat, Dict. de mat. méel., 1834.

sisymbrium amphibium, lat. des pharmac., Rosenthal, Synopsis.

raifort d'eau, m.. français, MÉRAT, Dict. de mat. médic., 1834.

radicine di chiana, ital., TARgIONI, Diz.

tajarinna, milanais, BaNfi, Vocab.

wassersenf, wasserrettiy, allem., Rosenthal, Synopsis plant. diaph.

waterkarse, waterklesse, flamand, DE B0, [A. DE C.].

wilde water-radys, flamand, DE GORTER.

\section{GARDA.MINE PRATENSIS (LINNÉ). - LE CRESSON DES PRÉS.}

\section{NoMs.}

nasturtium pratense, nasturtium agreste, flos cuculi, iberis, hiberis, lepidium minus, cardamine, sisymbrium cardamine, cardamine lalifolia, ancienne nomenclat., Bauhis, Pinax, 1671.

nasturtium sylvestre, anc. nomencl., DuE\%, 1664.

lepidium iberis, nomencl. de LAMARCK.

cresson des prés, m., faux cresson, m., cressonnette, f., cresson sauvaye, m., cardamine, f., cardamine des prés, $\mathrm{f}$., passerage sauvage, f., petite passerage, f., nasitort sauvage, m., chasserage, f., français.

cresson de Paros, m., français du XVIIIe siècle, Le préroyant jarlinier pour 1781.

crèssètte, f., Ruffey (près Dijon, rec. pers.

kerson d' pré, m. (Orne), LETACQ.

krinson, m., Pavs de Vaud, Bridel, Gloss. : Durhein, Idiot.

kerson das pras, m., cœur'son dé prêt, m., crohhon d' prés, m., crechon sauvaige, m., sauvaige her'son, m., courson doux, m., Vosges, Haillant, $F l$. pop.

cœurson de djadine, m., Ban de la Roche, OBERLin, Descript. cresson de saint Georyes, m., Doubs, BeAuquier, Voc.

saint Djôrdye, Bournois (Doubs), Rousser.

grä̈ssélou, m., Tar-et-Gar., LaGrèze-Foss.ıT, Fl. - Montauban, GateraU, Desc. 
grassillonassa, f., Luchon (Pyrénées), Sacaze.

graïssilou de prat, 111., Toulouse, Tournon, Fl.

créïsselou des prats, m., Tarn, Gary, Dict.

graïsselou de prat, m., creïsselou de prit, m., Sud-0uest du Languedoc,

Duboul, Las plantos.

gréssoun dè prat, m., yréssounasso, f., env. de Barbaste (Lot-et-Gar.), c.

par M. l'abbé L. DARDY.

crichilhou de prat, m., env. de Carcassonne, LAFfacie.

créissoun de prat, m., créïssounèto, f., provens. mod., Régus.

crètson de tsevuu, m., Montaigu-le-Blin (Allier), com. par M. F. Ducuon

DE LA JAROUSSE.

graïseloun, m., douceto, f., Forcalquier(Basses-Alpes), c. par M. E. PLAuchud. bouquet au loup, in., Aube, Des Etaxgs, Liste.

bouquin, m., Corniéville (Meuse), rec. pers. (Identif. pas absolument sûre). pain d'oiseau, m., environs de Langres, Des Etaxgs, Liste.

pain d'ayèlô, m. Leschères (Haute-Marne), c. par M. l'abbé Marchal.

pain d'oulié, in., Saulxures (Vosges), Haillant, fl. p.

pain d'alouette, m., Haute-Marne, c. par M. A. Daguin.

fourre de veau, m., Haute-Marne, c. par M. L. Avвriot.

pec à l'oiseau, ı1., Anjou, Desvaux, Fl.

hilt' d'ayuèss', hitt' d'onhai, f., Spa, LezaAck, Dict.

chil' d'ayass', f. pl. (excréments de pie), wallon, J. Feller.

soupe au vin, f., Cernois près Semur (Côte-d'Or), c. par M. H. M.ırLot. -

Côte-d'Or, Royer, Fl.

soupe en vin, f., Aube, Des Etangs, Liste. - Haute-Marne, c. par M. A. DAGUIN.

luit battu, m., Saint-Pol (P'as-de-Calais), c. par M. Ed. Ednont. - Pays de Bray (Normandie), JoRET, Fl. p.

lait battu, m. (la variété blanche), environs de Samer (Pas-rle-Calais),

c. par M. B. DE KeRHERvé.

trempee, f.. Aube, Des Etangs, Liste.

coucou, m., Eure, Robin, Dict. - Roumois (Normandie), Joret, Fl. p. pentecôte des prés, f., Normandie, JoRet, Fl. p.

pentecouéte, f., La Hague (Manche), J. Fleury, Pat. de La II.

saint-Jean, m., Ninville (Hante-Marne), c. par M. l'abbé Marchal.

sacre (1), m., Mayeme, comm. par un botaniste de la Mayenne.

fleur du tomnerre, f. (la variété cultivée flore pleno), français, Mall. sinfe rouge, f., Hesdin (Pas-de-Calais), rec. pers.

(1) La plante est ainsi appelée parce qu'elle fleurit à l'époque du Sacre (Fête-Dieu). 
meurampoule, f., env. de Langres, Des Etangs, Liste. - Haute-Marne, c. par M. A. Diguin.

tatapoulo, f., La Jonchère (Haute-Vienne), rec. pers.

cardinette, f., Bohain (Aisne), rec. personn.

claudinette, f., Haute-Marne, c. par M. L. AubRIot.

pingué, m. Offroicourt (Vosges), Halllant, Fl. p.

coquerette, f., couquerette, f., Haut-Maine, MonTEsson, Voc.

miguet bian, m., Montbéliard. Contejean, Gloss.

marest, anc. fr., J. Camus, Livre d'heures.

bran de Judas, m., Pays de Caux, Joret, Fl. p.

samevin, m., Pays de Madrie (Norm.). Joret, Fl. p.

chonfouin, (in prononcé comme en français), m., Le Buisson (Dordogne),

rec. pers.

graschan, Engadine, Grisons, Dunhem, Idiot.

billeri, viola da pesci, italien, TARGIONI, Di:.

raviz salvadeg, Vérone, P’ollixi, $F l$. ver.

erba celest, Veneria (Piémont), Colla.

créixens de prat, catalan, Costa, Fl. catalana; Vaýreda, Ciatal.

cardamina, portugais, Brotero, Fl. lus.

mastorzo dos pralos, galicien, Cuverro, Dicc.

stupitul cuculut, roumain, Br.s.dza, Limba bot.

iarba cocoshului (= herbe du coq), roumain, Ciнас, Dict.

gauchblume, ancien allem., DiEFExBacir, Gloss. merl. lat.

wiesen-schaumkraut, wiesenkresse, kukulisblume, wilde kresse, allemand. süsse brunnliresse, Autriche allem., Хемлісн, Polygl. Lex.

mattenliressich, schisgelte, ziegerbe, geltenblume, Suisse allem., PRITzEL.

hannotterblö, Altmark, DANNEIL.

grïttblom, heinotterblom, Altmark, PRITzEL.

spreenblome, Wildeshausen, Pritzel.

kiewitsblome, störkeblome, Frise orientale, Pritzel.

hennaïugli, chessali, canton de Saint-Gall, Pritzel.

schlafblume, storchblume, Prusse, Frischier, Preuss. IT.

kuckucksblome, Westphalie, LandoIs, Westph. Pflanzenn.

wilder liress, Carinthie, Lexer, kürnt. Lex.

liesblum, gêsseblum, wiseliacht, Luxembourg, J. WEBER.

loekoeks-bloem (1), flamand, Roucel, Fl. du norl. - Flandre orientale

(Brabant et Limbourg), c. par M. A. DE Cock.

(1) Là où le coucou (koekoek) a craché il naît une fleur de coucou (koekoeksbloem). ... Limbourg, comm. par M. A. DE COCK. 
koekkoeks bloem, water-violen, klein water ker's, flamand, DE GORTER. hoekoetsbloem, Flandre occidentale, DE Bo. [A. DE C.]. koekoeksbloem, wateriool, klein-waterkers, kievitsbloem, pinksterbloem hollandais, Oudemans. [A. DE C.].

kievits-bloem, Utrecht, DE GoRTER. pinxster, Frise hollandaise, DE GORTER. lustmoce, anglo-saxon, Cockayne, Leechdoms, etc. cuckoo flower, lady smock, (1) anglais.

cuckoo, cuckoo bread, cuctioo flower, cuclioo spit ( $\left.{ }^{2}\right)$, cuckoo's shoes and stockings, bogspinks, spinks, lady flock, bird-eyes, bonny birl een, lady milk sile, milksile, milk maid, milk maills, apple pie, bread and milk, may flower, headache, dans les divers dialectes de l'Angleterre, Britten, Plant-names.

milky maid, Devonshire, Friend, Gloss. of Dev. glourane, gleoran, lenymurry, irlandais, J. КЕоGн, 1738. [H. G.] billar grayan, irlandais, THRELKELD. [H. G.]. gleoran, plur na cubhaig (= cuckoo's flower), gaélique écossais, CanErox. [H. G.].

hydyf y waen (= la belle pousse du pré), hydyf blewog (la belle pousse chevelue), gallois, J. Morgan. [H. G.].

blodau'r gôg (= fleurs du coucou), gallois, Hugh DAviEs. [H. G.]. blodyn y gog (= fleur du coucou), gall. de Llanrwst, Jonx Williams [11. G.]. creç:on, breton, P. Grégoire. [E. E.]. kreson jardin, breton de Tréguier. [E. E.]. giøgeleyer, storkeblommer, danois, JENSSEN-Tusch. lirafnaklukia, lambaklukia, Islande, Jexssen-Tusch. brïsmagrïs, braxnablomster, suédois, IDEs. baekkekarse, gogeblomster, blejkurt, norvégien, IDEM. pènèn, pènènlia, tchèque, A. Müller, Synon. Nam.

(1) From the resemblance of its white fluwers to little smocks hung out to dry. Prior, Pop. names

(2) In the north of England the plant is known only by the name of cuckoo-spit, the rather inelegant cognomen being gained, no doubt, from the fact of almost every flowerstem having deposited upon it a frothy patch much ressembling the human saliva, in which is enveloped a pale green insect. Few north-country children will gather these flowers; they have a superstition that is unlucky to do so, and will tell you with the gravest countenance that the cuckoo has spit upon it while flying over. - Journal of Horticulture, May 4, 1876 (p. 355). BRITTEN, Plant-names. 
jèroukha bolotnaia (pâture des marais), russe, Schialmausen. [Th. V.]. gandro pipivai, lithuanien, JАСОВY, Lit. pflanzenn.

krasa, wende, Schulenburg, Wend. Volksth.

vodeni garbać, senożetna kres̈a, gardan divji, gardan, serbo-croate, ŠULeK, Jug. im. bil.

buminka, basque. [J. V.]

nuttu lirassi, finnois, Fellyan, Index.

jürri lilled, esthonien, IDEM.

ghassal, arabe algérien, Florian Pharaon, Voc.

qourrat el a'yn, resâd, arabe syrien, BERGGREs, Guide.

\section{SUPERSTITION.}

La Cardamine est la fleur favorite des serpents. Les enfants ne doivent pas la cueillir, sinon ils seront piqués dans l'année par un de ces reptiles. - Naintré (Vienne), rec. personn.

\section{GARI)A.MINE HIRSUTA. (LINNÉ.)}

cresson de vigne, m. Doubs, BEAuquier. - Villefranche (Rhône), cornm. par M. DÉRESSE (1).

cresson de muralle, m., quersonnette, f., Normandie, JoRET, Fl. $p$.

aiguilles à la Vierye, f. pl.. Cherbourg, JoRET, Fl. p.

creïssoun soouvagi, m., Forcalquier (Basses-Alpes), c. par II. E. PLAuchud.

billeri, ital., TARGioni, Diz. bot.

aruculicedda sarvagyia, sicilien, BIAncA, Flora d'Avola.

agrião menor, galicien, CuveIRo, Dicc.

ruige veldliers, néerlandais. [A. DE C.].

land cress, lamb's cress, anglais, BRITTEN.

berwr blewog ( $=$ cresson chevelu), gallois de Llanrwst, Jonn WiLliams.

[H. G.].

\section{CARDAMINE IMPATIENS. (LINNÉ).}

herbe au diable, f., Aube, DEs ÉTANGs, Liste.

billeri, italien.

(1) On mange les feuilles de cette plante, au printemps, dans le département du Rhône. (Comm. par M. Dénesse.) 


\section{CIARDAMINE AMARA. (LINNÉ).}

nasturtium aquaticum amarum, anc. nomencl.

cardamine amere, f., français savant.

herbe Saint-Taurin, f., Beaumesnil (Pays d'Ouche, Normandie) Joret. cresson amer, m., creusson, m., crohhon, m., kerson, m., caurson de

fontaine, m., crohhon d'ru, m., Yosges, Haillant, $F l . p$.

cardamindo amaro, italien, Nesnich, Polygl. Lex.

grassù, Brescia, Zersi.

mastuer'a amaryo, espagn., Colmeiro, Dicc.

cardama, rijnica, roumain, BBANDzA, Limba bot.

bitterkresse, allemand.

vandkarse, norvégien et danois.

jéroukha vodianaïa, (= pâture aquatique), russe, Schmalbausen. [TH. V.].

\section{GARDA.IINE ASARIFOLIA. (LINNÉ).}

coclearia d' montagna, Mondovi (Piémont), Colla.

cocleargia (perchè si usa come succedaneo della coclearia officinalis che manca nella nostra provincia), Brescia, ZERSI.

\section{CARDA.IINE PARVIFLORA. (LINNÉ).}

buconet, Druent (près Turin), Colla.

\section{DENTARIA (Genre). (LinNÉ.)}

coralloides, viola dentaria, dentaria, dentellaria, dentaria minor (1),

herbariorium alabastrites, anc. nomencl., Baunin, Pinax, 1671.

dentaria antıdysenterica, anc. nomencl. des officines, Rosevthal, Synopsis. dentaire, f., français savant.

récin' du din (racine de dent, allusion à la forme de la racine), Sourbrodt

(Pays wallon), J. Feller.

arabastro, f., Var, AMIc, Consid. s. Brignolles.

(1) On l'appelle ainsi pour la distinguer de la dentaria major qui appartient à une famille differente, celle des Orobanchos. 
dentaria, viola dentaria, italien du 16 e siècle, J. Camus et 0 . Penzıg, Illustrazione del erbario estense, p. 26.

dentanèla, Brescia, Zensi.

dintaria, sicilien, MorTLLLARo.

zankraut, schuppenwurzel, anc. allem., DIEFENBACH, Gl. med. lat. zahnwurz, allem., Rosenthal, Synopsis.

tandkruid, hollandais. [A. DE C.].

leadwort, toothwort, anglais, J. KEOGH, 1735.

tooth cress, tooth violet, coral-wort, anglais, Prior, Pop. names.

slanufikal (= santé des dents), irlandais, J. KEOGH, 1735. [H. G.].

lhachichet es senain, arabe syrien, BERGGRen, Guide.

kaghendzni, arménien, Alishan. [Er. L.].

\section{DENTARIA BULBIFERA. (LINNÉ.)}

dentaria bulbifera, dentaria baccifera, dentaria heptaphyllos, anc. nomenel.

Baunin, Pinax, 1671.

coltçishor, roumain, Brandza, limba bot.

grimwurz, grimwur:l, Autriche allem., F. IÏ̈FER et KRONFELd.

scluppenwurz, Thuringe, Pritzel.

deintlys (= herbe aux dents), gallois, Hugh Davies. [H. G.].

jassenetzi, petit russien, c. par M. Тн. VoLkov.

\section{DENTARIA DIGITATA. (LaMaRci.)}

dentaria pentaphyllos, anc. nomencl., Bauhin, Pinax, 1671.

cresson de bois, m., Doubs, BeAuquier, Voc. du D.

pertafillu fausu, m., sicilien, Lagusi, Erbuario, 1744.

tavarau, Trévise, Saccardo, Fl. Trev.

blauer scharniggl, Carinthie, ZWANZIGER, Verzeichn.

babie zẹby (= dents de vieille femme), polonais, LINDE.

\section{DENTARIA PINNATA. (LAMARGK.)}

viola capraria, italien vulgaire du XVIe siècle, J. Casus, Historique des Herbiers, 1885, p. 24.

dentanela, Brescia, MeLchioRi.

canudera, Vall de Nuria (Catalogne), VAyredA, Catal. 


\section{DENTARIA ENNEAPHYLLOS. (LINNE.)}

enneaphyllon, anc. nomencl., Batur, Pinax, 1671.

berysanikel, Suisse allem., Pritzer.

samikl, Bavière, IDEM.

weissa sánigel, weissa sennig'l, Autriche allem., IIÖFER et KroNFeLd. weisser scharniggl, Carinthie, ZWANZIGER.

\section{NOTOGERAS G.NARIENSE. (R. BROWN.)}

trevol reventon, Canaries, Wesв, Hist. nat. des Can.

IIESPERIS IATRONALIS. (LINNÉ). - LA JULIENNE.

\section{1. - Noms de CETte plante.}

šrsois (1), grec ancien xíб:os, grec mod., Schmid, Nouv. dict. grec mod., 1838.

hesperis hortensis, viola matronalis, viola purpurea (2), viola alba, viola damascena, leucoinm purpurenm, leucoium alburn, anc. nomencl., Bauin, Pinax, 1671.

flos gariofilus, anc. nomencl., C.ES.lLPinus, De plantis, 1583. girollèe, f., français.

riolette des dames, f., franç., Dodoneus, Herbarius, 1608. - Cotgrave, A french dict., 1650.

violier des dames, m., roquette des jardins, f., français, Fillassier, 1791. giroflee des dames, f., franç., Dodon.eus, Herb., 1608; Vallot, Hortus regius, 1665; BaIllx, Hanuel du jardinier, 1825, II, p. 182. violette de Damas, f., franc., Cotgrave, A french dict., 1650. - Dod0N.EUS, Herb., 1608.

violette des matrones, f., matrones, f. pl., franç., Cotgrave, 1650. matrône, f., wallon, J. Feller.

matronle, f., La Reid, Hautregard (Belgique wallonne), J. FELLER. djoley' matrône, f., (la variété de couleur violacée), wallon. J. FELLER. violée matrône, f., (= matrone violette, la var. violette), wallon, J. Felleb.

violette musquée, f., français, Montsainct, Le jardin senonois, 1604.

(1) On l'a appelée ansi du mot $\hat{\sigma} \sigma \pi \varepsilon \rho \circ \varsigma$, soir, parce que c'est le soir qu'elle répand le plus d'odeur.

(2) Il y a deux variétés principales, l'une d'un rouge pourpire, l'autre d'un beau blanc. 
giroflée d'Angleterre, f., franç., DE Cinanvalos, Manuel des champs, 1765, p. 59 .

géroflée musquée, f., gérofleee d'Angleterre, f., LIGER, Le jardinier fleuriste, 1718 , t. I, p. 54 et p. 118.

juliane, f., français, Vallot, Horlus regius, 1665.

juliane, f., (la giroflée musquée double), P. Monsn, Rem. s. la cult. des fleurs, 1694, p. 6.

juliane des jaidins, f., français, Fillassıer, 1791.

julienne, f., français.

jouliano, f., Var, Hanry, Calol.

juillenne, f., Loiret, Eure-et-Loir, comm. par M. J. POฉLET.

joulieto, f., Var. Axic, Consil. sur Brignoles.

julio, f., Ballon (Sarthe), rec. pers.

pentecótes, f. pl., Normandie,, JoRET, Fl. p.

carafée, f., Haute-Marne, comm. par M. A. DAGUin.

yiroflée rose, f., Idem, IDEM.

lamns, m., français, Valenciennes, Hécart. - Saint-Pol (Pas-de-Calais),

comm. par Eb. Ediont, - wailon, Feller.

muguél, m., Tulle (Corrèze), Figreac (Lot), G. DE Lépinay, Noms.

blanc musque, m., Saint-Pol Pas-de-Calais), comm. par M. Ed.

EDMONT.

cassolette, f., français, BALly, Manuel du jardinier, 1825, t, I, p. 182. cassoleto, f., Montauban, (iaterau, Descr. des plantes.

cassouleto, f., Sud-0uest du Languedoc, A. Diboct. - Aude, Laffage. -

Gard, comm. par II. P. Fesqcet. - Forcalquier, com. par M. E.

Placchud.

cassouleto salbatjo, f., Sud-0uest du Langued., A. Duboul, Plantos. -

gascon, Noulet, Flore.

yirarda, f., Suisse romande, Bridel. - Lyonnais, Puitspelu.

givarde, f., Centre de la France. Boreav, Fl. du Centre. - Lyon,

Molard, Le manvais langage corrigé, 1810.

girardo, f., env. d'Aix en Prov., Garidel.

Igrrardo, f., Haute-Loire, ARnaud, Flore de la Haute-Loire.

bombarle, f., Yonne, Jossier, Dict.

saint-Jacques, Normandie, Joret. - Orne, LetacQ.

beurrée, f., français, LAMarck et DE CANDolle.

thanta-polè (avec th anglais), Cervant Haute-Savoie), rec. pers. (? - L'identification n'est pas absolument sûre'.

aragonne, f., Marquion (Pas-de-Calais, rec. pers. - Samer (Pas-de-Cal.), comm, par M. B. DE KerIIERvÉ.

cloquelourde, f., Pays d'Auge (Norm.), Jonet, Fl. $p$. 
coquelourde, f., Pont-Audemer (Eure), Robin, Dict. - Normandie, Joret. - Orne, Letace.

conclourde, f., Franqueville (dans le Roumois, Norm.), Joret, Fl. p. mahoun, m., Sud-0uest du Languedoc, А. Duboul, Plantos. croix blanche, f., croix rouge, f., Haut-Maine, Movtesson, Voc. croix de Jérusalem, f., Olloy (Belg. wallonne), J. FELLER. crèchado pitiouno, f., Le Buisson (Dordogne), rec. pers. clabete, f., clabetine, f., béarnais, Lespy, Dict. béarn. viola a ciocca svizzera, viola matronale, italien, RAMbelu, Vocabol. domestico, Bologna, 1850.

riolucciocco forestiero, violacciocco svizzero, ital., Targioni, Dis. bot. viulè d'Spayna, viulè sarraj, giulien-e, piémontais, Colla. vioèula matronala, Parme, Malaspina, Voc.

viola madrunêla, romagnol, MoRri.

daminna, milanais, BANFi, $10 c$. samberi, Trévise, Saccardo, Fl. trev. juliana, espagn., Colmeiro, Dicc. goiveiro incarnado, port., Brotero, Fl. mirodénie (= parfum), roumain, СıнAc. damastblume, all :mand, Grims, IVört. damaschteblum, Luxembourg, J. WEBER. damaschle, Prusse, Treichel, Volksth. aus d. Pflanzenw. dammast'n, vijôl maternaol, Altmark, DAxNeıL. medaschlie, Prusse, Frischbier, Preuss. Wört. matronalviole, matronenblume, frauenviole, allemand, Хемкісн. matronalfeigel, Carinthie, Zwaxziger, Verz.

vigole maternaole, nachtvigöllen, Westphalie, Laxdors, Westph. P . nachtveil, nachtviole, allemand.

pfingstveiali, cant. de Saint-Gall, Wartmans, Volksbot.

pfingstnägele, Alsace, KirRsChlEGER, Fl. d'Als.

mastbloemen, damasbloemen, anc. flam., Dodox.eus, 1644 [A. DE C.]. damastbloem, nachtviolier, flamand et hollandais. [A. DE C.]. queen's gilloflowers, rogues gilloflowers, close sciences, damask, dames violets, damask violets, anglais, CotGRave, A french äict., 1650. white rocket, whitesun gilawers, West Somerset. Elworthy, West Som. word-book.

whissun gillofer, Shropshire, Jackson, Word-book. siney, Plymouth, Briggs, Flora of Plym., 1880, p. 19. julyennès, breton, l'. GRÉG0IRE. [E. E.]. nattfiol, suédois. damaskblommer, himmelsprækker, danois, JENSSEN-TusCH. 
večernica (= plante du soir), serbo-croate, Šulek.

notchnä̈a fialka (= violette nocturne), russe, Schmalhausen. [TH. V.]. notschnáïa krassotá, russe du gouvern. de Voronèje, c. par M. A. DiKAREv. 2. - On dit : Blanc comme un damas.

Valenciennes, HĖCART, Dict. rouchi.

3. - Nachdem bei dem Kreuzestode Christi alle Blumen bereits in tiefem Schlafe versunken waren, blieb nur noch die Nachtviole wach und sandte zur Erquickung dem sterbenden Gottmenschen balsamische Düfte empor. Als Lohn dafür entfaltet sie seit diesem Tage auf Gottes Geheiss immer zur Nachtzeit die Pracht ihres Kelches.

Autriche allemande, communication de M. F. BRANKY.

4. - Si vous donnez un plan de matrone, celles qui vous restent au jardin meurent. Pour éviter cet ennui, il faut simuler une vente de la plante et se faire remettre quelques centimes en échange.

Verviers (Belgique), J. Feller.

5. - Celui qui donne une matrone à un autre, meurt. Il faut simuler une vente ou un échange pour échapper à ce malheur.

Herve et Liège (Belg.), J. Fellen.

IIESPERIS ACIRIS. (FORAKAL).

meddiul, sefeyry, arabe égyptien, DELILE, Fl. aey. ill.

\section{MALGOLMIIA MARITIMA. (R. BROWN).}

cheiranthus maritimus, nomencl. de Liswé.

giroplée de Mahon, f., julienne de Mahon, f., gazon de Mahon, m., julienne de Chio, f., français.

mahonille, f., français, B.ıLLY, Manuel du jardinier, 1895, t. II, p. 151 ;

Le bon jardinier pour 1827, p. 627.

gazon, m., Pays de Caux, JoRet, Fl. p.

yaミoun de Paris, m., Bouches-du-Rhòne, Villeneuve, Statist.

yazou d'Oulando, m., languedocien, Poumarède, Manuel; A. Duboul,

Plantos.

violacciocca di Ilaone, ital., TARGIONI, Diz.

baichetto, Gênes, Casaccia, Diz. gen.

aleli de Mahon, aleli del papa, espagn., CoLmeıro, Dicc. 
prado, catalan, Costa, Fl.

yasó, Baléares, Marès, Catal.

devotekatoen, flamand, DE Bo, Westel. ldioticon.

gaziun, Malte, Delicata, Fl. melit.

\section{IIALCOL.MIA LEGYPTIACA.}

dakhayân, qoreyn, arabe d'El-'Arich (entre l'Egypte et la Syrie), Ascherso.

(i la suite de l'lllustration).

el maroûdjé, el hamî, arabe du Sahara, Duverrier, Les Touareys.

almaroûdjet, berbère témâhaq, IDEM.

\section{LEPTALEUM FILIFOLIU.M. (DE Gandolle).}

qeseysah, arabe égyptien, Ascherson et Schweinfurth.

AlLIARIA OFFICINiLIS. (DE Gandolee). - L'AlLIAIRE.

alliaria (1), alliastrum, rima marina, rima maria, thlaspidium cornutum, anc. nomencl., Bavhis, Pinax, 1671.

scordotis, anc. nomencl, Fuchsius, 1546.

alliaris, pes asini, pes asinimus, anc. nomencl., Fuchsils, 1540 ; LiNOCIER, Hist. des pl., 1584.

erysimum alliaria, nomencl. de LiNvé.

hesperis alliaria, nomencl. de LAMARCK.

alliaire, f., français, A. Pindeus, Ilist. pl., 1561 ; Livocier, H. des pl.,

1584; Duez, Dis. it. fr. 1678.

julienne alliaire, f., vélar alliaire, m., français.

herbe à l'ail, f., Centre, Jalbert, Gloss. - Allier, c. par M. E. Olivier. herbe aux aul.x, f., français, Cotgrate, A french dict., 1650: DuEz, Diz.

it. fr., 1678.

erbo d'al, f., Toulouse, Tourion, Fl., - Aude, Laffage. - Sud-Ouest du

Languedoc, A. Duboel, Plantos.

lerpe ì z’aux, f., Valenciennes, Hécart, Dict. rouchi.

yèp' au.x as, f., Wallon, J. Feller.

herbe aux aillets, f., français, DuEz, Diz. it. fr., 1678.

ä̈ets, in. pl., Liège, J. FelLEr.

(1) Les feuilles de cette plante, quand on les écrase, répandent une forte odeur d'ail. 
erbo d'alh, f., alhas, m., Barbaste (Lot-et-Gar.), c. par M. l'abbé L. DARDY. erbo de laïet, f., Environs d'Avignon, Palun, Cat. erbo d'aièt, f., Forcalquier, c. par M. E. PLAdchud. aillaut, m., Haute-Marne, comm. par M. A. DAguin. ailladés, f. pl., Luchon (Pyrénées), J. SAcaze. aury, ory, Anjou, Desradx, Fl. - Mayenne, c.par un botan. de la Mayenne. rumat, m., Saint-Pons (Hérault), Barthès, Gloss. bot. poulo gracho, Corrèze, fr. DE LéPINAx, Noms des $\mathrm{pl}$. herbe sans pareille, f . Pamproux (Deux-Sèvres), c. par II. B. Solché. reutche, f., Plancher-les-Hines (Haute-Savoie), Poulet, Voc.

cadotte, 1., herbe ai lai griotte, f., (= herbe au foie), Montbéliard, Contejean, Gloss.

dchotte de chnache, f., Ban de la Roche, H. G. OBErlin, Descr. pied d'asne, m., français du $16^{\circ}$ s., L. Fuchs, Hist. des pl., 1558. alliaria, erba alliaria, lunaria salvatica. piè d'asıno, ital., TARgioni, Diz. agghialora, aghialora, sicilien, CuPAsi, Hort. cathol.. 1696; LAgUsi, Erbuario, 1742.

erba joèule, Parme, Malaspixa, Voc. rimamíria, ital., DuEz, Diz. it. fr., 1678. herva alheira, portug., Вкотеко, Flor'a lus. erba d'o allo, galicien, VALLADARES. jarbe de lingoarie, roumain de Transylvanie, Fusz, Trivialnamen, 1848. usturoitca, roumain, BRANDZA, Limba. knoblauchs-hederich, allemand. knoblauchkraut, ramschelwurz, Silésie, PRITzEL. blöderkraut, Eifel, Wintgen. Veq. I. Eifel. blôderkraut, Luxembourg, J. WEBER. blatterliraut, Eifel, Wintgen, Veg. di Eifel. salzkiraut, Prusse orientale, Pritzel.

lauchel, Prusse, Frischier, Preuss. Wört. knoblengswurz, linoblengsliroclit, all. de Transylvanie, Fusz, Trivilnamen. loock sonder loock, ancien flamand, DoDoxaEcs, 1644. [A. DE C.]. look zonder look, lookraket, flamand et holland. [A. DE C.]. leaccerse, anglo-saxon, Cockarse, Leechloms, etc. yarlick-wort, anglais. jack by the hedlye, jack in the bush, beggar-man's oalmeal, saulce alone, english treacle, anglais ( $\left.{ }^{1}\right)$, BRITteN.

(1) Sur les noms anglais de cette plante, roy. Notes and Queries, $2^{\circ}$ ser. t. XII, p. 148 et p. 338 . 
bocuineall ( $=$ chandelle de vache), gairleog coillah ( $=$ ail des bois), irlandais, J. Кеосн, 1735. [H. G.].

bo coinneal (= cow-candle), yarbhraitheach (= rough, threateniny), gairleach collaid, gaélique écoss., CAmenon. [H. G.].

troed yr assen (= pied de l'âne), (g)arlleyog (= alliacé), gallois, J. Davies, 1632. [H. G.].

garllegog, garlleg-ferwr (= cresson-ail), gallois, HuGH DaVIEs. [H.G.].

llawer y coed (= sol du bois), gallois, J. Morgan. [H. G.].

garleyd keylley (= ail des bois), mannois, J. KELLY. [H. G.].

hvitlöksörtt, suédois.

yaffelkäl, danois.

tchesnotchnaia traia (= herbe de l'ail), russe, Schislн. [Тн. V.].

iesnaček (petit ail), česnekol'a bylina, tchèque, A. MüLler, Syn. Namen. c̈es̈njaka, česen borstni, hren rogati (= raifort cornu), kremuć, lučina,

lucica, lukovac, serbo-croate, Šulek.

baratzuri belhar, basque. [J. V.].

foghayyma línya, magyar, Fusz, Trivialn.

hhachichet et toumiyye, kerât, arabe syrien, BERGGren, Guide.

chestoramaïr (= mère de l'ail), anc. arménien, Alishan. [ER. L.].

\section{ERYSIIUU CHEIRANTHOIDES. (LINNE).}

viola lutea sylvestris, anc. nomencl., Tragus, 1552.

giroflée sauvage, f., fausse yiroflée, f., carafée sauvage, f., fausse cara-

fée, f., Haute-Marne, comm. par M. A. Digurn.

violacciocche salvatiche, ital., TARGIONI, Diz.

vioele salvadeghe, Brescia, Zersi.

schotendotter, Silésie, Pritzel.

yroote wilde ker's, flamand, DE GORTER.

jeltouchnik (= plante de la jaunisse), russe, Scmmalhausen. [Ti. V.].

sweris, didaszwa, lithuanien, ЈАсову, Lit. Pfl.

\section{ERYSIMUM ODORATUM. (EHRHART).}

giroflée sauvage, f,, Aube, Des Étangs, Liste.

\section{ERYSHMUM MURALE. (Desfontaines).}

roquette, f., Valenciennes, HÉcart.

torlelo, f., Apt, Colignon, Fl. d'Apt.

erba diavola, ruchetta, rucola, salterelli, ital., TARgrosi, Diz. 
ERYSIMUM OCHROLEUGUM. (De Gandolle).

iaounclo, f., Sud-0uest du Languedoc, A. Duboul, $\mathrm{Pl}$.

ER YSIMU.I HIERAGIFOLIUM. (LINNÉ).

grespignáccio, ital., TARgioni, Diz.

SYRENIA SESSILIFLORA. (LEDEBOUR).

jeltouchnik (= plante contre la jaunisse), russe, Schmalhausen. [TiI. V.].

SIS YMBRIUM OFFICINALE. (SGOPOLI). - LE VÉLAR.

غे '́r

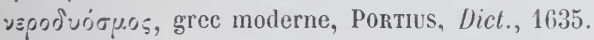

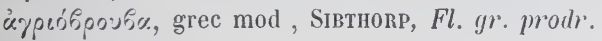

irio, latin, Pline.

erismon, eruca gortina, eruca gorgona, nomenclat. du moyen âge, Mowat, Alphita.

eruca geratina, tortela, vellarum, nobilis eruca, erision, nomenclat. du moyen âge, Diefenbach, Gil. m. lat.

erysium, anc. nomencl., Proprietez des simples, 1569, p. 59.

erysimum Diocoridis, sinapi, erysimum vulgare, hierobotane fremina, verbena foemina (d'après Tragus), verbena mas (d'après Fucissus), verbena recta, anc. nomencl., BauHis, Pinax, 1671.

erysimum, rapislrum, sinapis sylvestris, anc. nomencl., LINocIER, Ilist. des $p l ., 1584$.

erysimum officinale, nomencl. de LinNÉ.

vélar, m., tortelle, f, herbe au chantre $\left.{ }^{2}\right)$, f., français.

julienne jaune, f., BaIll, Manuel du jardinier, 1825, t. II, p. 182.

ieppe di chantre, f., Vosges, Haillant. Fl. p.

(1) Nais non l'èp'úrųoy de Théophraste, qni est sans doute l'hibiscus esculentus. (Eug. Fournier, Réforme de la nomencl. bot., dans Journ. des sav., 1880). Voyez encore à ce sujet : Bauhis, Pinax, 16 1 1, p. 100.

(2) On se sert de cette plante contre l'enrouement, l'aphonie, les maux de gorge. 
erba daou chantré, f., Gard, Rouger.

erbo dé cantaïré, f., Barbaste (Lot-et-Gar.), com. par M. l'abbé L. DARdy. erbo dès chantres, f., Villefranche de Lauraguais (Haute-Garonne), conm.

par M. P. FAGOT.

yèbe di chanteu, f., mostâde di hàye, f., wallon, Semertier.

mostotte th hồy, f., moustôte dés hay' ( moutarde des haies), f., rèvlouhhe, f., v'erveine, f., vèlvône, f., v'erlin-n', f., vètvöne, f., djène verveine (= verveine jaune), $\mathrm{f}$, djène marjolaine $1=$ marjolaine jaune), f., verveine des hommes, f., fleûr du mossieu, f.. (par opposition ì la fleûr de malame qui est la verveine officinale), fleûr dè mocheu, wallon, J. FELLER.

tourtelo, f., Var, Awic, Consid. sur Brignolles.

ravanisclé, m., Gard, PouzoLz, Fl. du Gard.

tzompil, m., Le Buisson (Dordogne), rec. pers.

ranth (avec th anglais), Cervant (Savoie), rec. pers. (? - Identification non absolument sûre).

erbo dé santo Barbo, f., Forcalquier, comm. par M. E. Plauchud.

cascèllora, erba cornacchia, erba crociona, erba grana maschio, erisamo,

erisimo, irione, rapa saliatica, rapino, senapaccia salvatica, verbena muschiata, ital., TARGion, Diz.

spinass sarvaj, Cunćo (Piémont), Colla.

radeci falsi, radeci salvadrghi, Vérone, Pollın, Fl. ver.

barbèna, Trévise, Saccardo, Fl. trev.

jerve d'amore, Abruzzes, Finamore, Bot. pop. abruzz.

cacacàni, sicilien, Branca, fil. d'Avola.

trion, milanais, Banfi, Voc.

trione, lombard, Scandagatti, Erbe dei prati asciutti, 1794.

lassanèddi, sicilien, Mortillaro.

feugyia de navôn, gènois, Casaccia, Diz.

yerva de san Alberto, espagn., Colmeiro, Dicc.

rabanissas, Vall de Nuria (Catalogne), Valreda, Catal.

rinchôo, portug., Brotfro, $\mathrm{Fl}$.

erba dos chantres, galicien, Cuverro.

bubolnic, roumain, Cinac, Dict. daco-roman.

frunse roinkriului, raptjitze, roumain de Transylv., Fusz, Trivialnamen. frunlça voinicului, brincutça voinicului, roumain, BRANdza, Limba bot. weysenff, ackierköl, anc. all., DiefEnBACH.

herlerich, wegesenf. wilder senf, lireuskraut, allemand.

gelbes eisenliraut, Silésie, P'RITZEL.

liök, kötge, Holstein, PRITzEL.

steenraliet, gemeene raket, hollandais. [A. DE C.]. 
hederick, flamand, Rovcel, Fl. du nord.

duchtiy kruid, flamand du pays d'Alost. Se donne aux vaches pour les rendre duchtig, pour les faire entrer en rut), comm. par II. А. DE Коск. tynthare, anc. anglais, Mowat, Alphita.

hedlge mustard, anglais.

crambling rocket, anglais, PRIOR, Pop. names,

lucifer matches, bankcress, anglais, BRITTEN.

melisse, lusanore, irlandais, J. KE0GH, 1735. [H. G.] .

meilise, gaélique écossais, CaMeroN. H. G.].

$\operatorname{arfog}(=$ armé), gallois, Hugh DAvies. [H. G.].

cedw'r gwrych (= moutarde de la haie), gallois de Llanrwst, Јонм

Williams. [H. G.].

güéerès, breton, P. GRÉGoIRE. [E. E.].

gwéérez, breton, LıÉGARD. (de guea = tordre). [E. E.].

dufvekål, vägsenap, suédois, JENSSEN-Tusch.

hořcice planá, hulevnik, křez, tryzel, gorušica divja, tchèque, A. MüLLER. soukhorebrik (= ayant les côtes sèches), petit russicn, Schyalhauses.

[Тн. V.].

gortschitza polewaja, russe, FALK, Beyträge, 1786.

pelırkones, letton, Ulmans, Lett. Wörterb.

mendazhi, basque. [J. V.].

nöstényfï, magyar, Fusz, Trivialnamen.

bsima, Malte, Delicata, Fl. melit.

thrayou chot (herbe des petits des oiseaux), arménien, ALISHAN. [Er. L.]

\section{SIS YIIBRIUM SOPHIA. (LINNÉ).}

nasturtium sylvestre, seriphium germanicum, seriphiuin absynthium, thalictrum, sophia chirurgorum (1), sophia duplex, accipitrina duplex, anc. nomencl., Bauhin, Pinax, 1671.

sophie, f., français, Cotgrave, Fr. dict., 1650.

talictron des boutiques, thalitron, sagesse des chirurgiens, sisymbre à

petites fleurs, m., français d'origine savante.

sneide, Romont (Vosges), Haillant, Fl. p.

talitro, pigamo, verdemarco, ital., Nemich, Polygl. Lex.

erba falcuna, Brescia, ZERSI.

(1) Ce nom est dû à la haute opinion que l'on a eue pendant longtemps de son efficacité comme vulnéraire. 
talitro, arnacho, espagnol, Neмnich, Polygl. Lex.

ajenjo serifio, espagn., Colarerro, Dicc.

erba sophia, portugais, Nexnich, Polyyl. Lex.

sophienkraut, sophiekraut, allemand.

sophrankraut, allemand, Rose.Tthal, Synopsis.

sophienrauke, bluthraut, allemand, A. MüLLER, Syn. Nam.

wurmsamen. wurmkraut, grosses besenkraut, allemand, Neмxici.

wellsamen, Bavière, Hesse, Pritzel.

habichlshraut, Silćsie, IDE.y.

fiene grete, Frise orientale, Focke, Pflanzenn., 1870.

besenkraut (1), Prusse, Frischbier, Preuss. Wört.

vuur-kruid, sophie-kruid, fie-kruid, flamand, DE GoRTER.

fiekruid (abréviation pour Sofieliruid, hollandais.

lax-wort, flix wort, flix weel, anglais, Cotgrave, Fr. dict., 1650.

flux-weed, anglais, Prior, Pop. names.

sophiegräs, barbärlälija, hunddill, suédois, JENSSEN-Tuscr.

teineal Muire, irlandais, Stokes, dans Revue celt., IX, p. 233. [H. G.]. gorrullumine, finelmuire, irlandais, J. KEOGH, 1735. [H. G.].

finel Muire, irlandais, THRELKELD (H. G.].

fineal Mhuire (= fenouil de Marie), irlandais, 0’ReILlY. 'H. G.].

berwi y fam (= cresson de la mère), piblys (= pipe-weed), gallois, Hugi

DAvies. [H. G.].

piblys, gallois, J. MoRGAN. [H. G.].

barbeerforstand, bartskjærs visdom, hundesennep, danois.

vild asparres, norvégien.

hulevnili, tchèque, A. Müller, Syn. Nam.

gorušica divja (= cresson de Jérusalem), serbo-croate, ŠulEk.

guliavnik, strutschatoï guliavnik, russe, FAlk, Beytrüge, 1786.

kanja-fü, seb' sorraszto fü, magyar, NEмxich.

SIS YMBRIUM IRIO. (LINNÉ).

rouqueto fero, f., envir. d'Avignon, Palun, Catal.

rouqueto salbatjo, f., Toulouse, Tounnon, Fl. - Sud-Ouest du Languedoc,

A. Duboul, $P l$.

rouqueto jaouno, f., rouqueto soouvajo, f., Forcalquier, com. par M. E. Plauchud.

(1) Besenkraut, Kraut aus welchem Besen gebunden werden. (FrIschBIER). 
erba irida, ireos, senapaccia salvatica, ital., TARGIon, Diz.

pisciacani, Mirto (Sicile), Cupani, 1696.

mazzareddi amari, lapazzani, Sicile, CUPANI, 1696.

matacandil, espagnol, Colmeiro, Dicc.

apagallums, catalan, Costa, Flora.

london rocket, anglais, PRIOR, Pop. names.

schutsemff, allem., EBEr et PEucer, Appellationes, 1556.

berwr caersalem (= cresson de Jérusalem), gallois, Hugi Davies. [H. G.]. lebsân el bh'aïr, arabe de Constantine, Prax, Plantes de Constantine, 18.j0. kernounech çifi, arabe de Tlemcen, Joundan, Flore de Tlemcen.

\section{SIS YMBRIUM POL YCERATIU.I. (LINNÉ).}

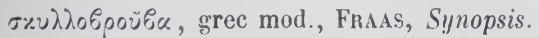

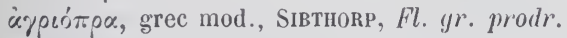

cimiciara, sicilien, BIANCA, Fl. d'Avola.

SIS YMBRIUM ASPERUMI. (LINNÉ).

creissous bastars, m. pl., Le Vigan (Gard), Rouger, Topographie, 1819.

\section{SISYMBRIUII GINEREUM}

gherîra, arabe de Biskra, Prax, Pl. de Const., 1850.

SIS YIIBRIUM ORIENTALE. (LINNÉ).

eureillè de lèbre, f., Issoire (Puy-de-Dôme), comm. par feu J. Bareire.

$$
\text { SIS Y.MBRIUII ALTISSIMUM. (LINNÊ). }
$$

rohatchka (= la cornue), petit russien, Schmalhausen. [TH. V.]. 



\section{LISTE PAR ORDRE ALPHABÉTIQUE}

\section{DES AUTEURS CITÉS}

\section{W.INS LE PREMER VOLUME DE LA FLORE POPLLAIRE}

Ackermann, Parabilium medicamentorum scriptores antiqui;... L. Apuleii de medicaminibus herbarum liber. Norimbergae, 1788.

ADAM (L.), Les patois lorrains. Nancy, 1881.

ADIY, Glossary of words used in the neighb. of Sheffield, 1888.

Alisiran, Haïboussali = Les noms arméniens des plantes). Venise, 1895. en arménien .

Alonso (J.), Dies pririleyios para mugeres preñadas, Alcala, 1606, in-4․ A la fin de cet ouvrage se trouve un dictionn. des termes d'hist. nat.)

Ambrosinus (H.), Plyytologia, Bononiae, 1666, in-folio.

AMIC (A.', Considerat. topogr. sur Brignoles (Var), 1837.

ANdrews (J. B.), locabulaire mentonais. Nice, 1877.

Ancuirlara (L.), Semplici. Venise, 1561, in-12.

Apuleius, De medicaminibus herbarum. Edité par Ackermans, 1788.

(Voyez ci-dessus Ackermann.

Arvald (J. A. M.), Flore de la Haute-Loire. Le Puy, 1825.

Ascherson(P.), Die aus dem mittlern Nordafrica, dem Gebiete der Rohlf's'schen, Expedition nach Kufra beliannt gewordenen Pflanzen (dans G. RoHLFs, Kufra, Leipsig, 1881.)

Ascherson P. et G. Schweinfurth, Illustration de la Flore d'Egypte dans

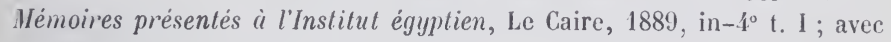
un Supplement dans le t. II.)

AzaIs, Dictionn. des idiomes romans du midi de la France. 1877.

BANFI (G.), Vocabolario milanese italiano, 1870.

Barbier de Meynard, Dictionnaire tur'c francais, 1885.

Barbosa, Dictionarium lusitanico-latinum, 1611.

Barthès (M.), Glossaire botanique de l'arrondissement de Saint-Pons (Hérault). Montpellier, 1873.

Baudoun (A.), Glossaire du patois de la forêt de Clairvaux (Aube). Troyes, 1887.

Bıuhin (G.), Pinax theatri botanici. Basileae. 1671.

Baunin (J.), De plantis "divis sanctisque nomen labentibus, Basileae, 1594, in-8. 
Beauchet-Filleau, Essai sur le patois poitevin on petit ylossaire des mots usités dans le canton de Chef-Boutonne. Melle. 1863.

Beavquer (Сн.), Vocabulaire des provincialismes usités dans le Doubs, Besançon, 1881, in-8.

Bergen (F. D.), Popular american plant-names (dans Journal of mmerican Folk-Lore, 1893.

Berggren, Guide francais-arabe vulgaire, Ipsal, 1811.

Béronie (N.), Dictionn. du patois du Bas-Limousin. Tulle, in-4o.

Bravca (G.), Flora dei dintorni d'A rola dans Atti dell'Accademiu giveniu di scienze naturali di Catania. (a paru par fragments de 1842 a 1857.

Billerbeck, Flora classica, Leipsig, 1824.

Boerio (G.), Dizionario del diuletto veneziano. Venezia, 1856 , in-4 ${ }^{\circ}$.

Böhner (D. G. R.), Commentationes øeconomico-medico-botanicae. Vitelergae. 1792.

B̈̈нтылgк (0.), Sanscrit Wörterbuch. 1855.

Boissier (Edm.), Voyaye hotanique dans le midi le l'Espagne, 1839-1845. in- $4^{\circ}$.

BoNxet Ed.), Una nomenclatura medico-botanica estratta da un codice del secolo $I X$, scritto nell' Italia settentrionale. (Estratto dagli Atti del Conyresso botanico internazionale, 1892, in-8; 11 pages.)

Borao (G.), Diccionario de t'oces arayonesas. Zaragoza. 1859, in-8.

Boreau, Flore du centre, 1857.

Botcherie (1.), Additions an dictionnaire de Littré, Lexicologie iotanigue, ele., in- $8^{\circ}$ de $3 i$ pages.

Boucherie (ג.), Patois de la Saintonge, 1865.

Boyer de fonscolombe, Calendrier de Fane et de Filore pour les environs d'Aix: (dans Mem. de l'Acad. d'Aix, 1844-1845.)

Bracciforti (A.), Flora piacentina, Piacenza, 1877.

BRACHet (F.), Dictionn. du patois savoyard parle duns le canton d'Albertville, 1883.

Brandza (D.), Prodromul florei române, Bucuresci, 1879-1883.

Brandza (D.), Limba botanica a tecranului românu (dans Columna lui Traianu, Bucuresci, 1882.)

Bremisches Wörterbuch, Bremen, 1767-1771, in-8.

Bridel, Glossaire du patois de la Suisse romande. Lausanne, 1866.

Briggs, Flora of Plymouth, London, 1880.

Britten (J.) and R. Holland, Dictionary of english plant-names, London. 1880.

Bronon (Jean), De stirpibus, 1541, in-12.

brotero (F. A.), Flora lusitanica, Lissibonac, 1804.

Brom (A.), Descrizione botanica delle campagne di Barletta, Sapoli, 1857. 
Caasalpinus (A. De plantis libri XVI. Florentiae, in-4º, 1583.

[Caesalpinus], Th. Caruelii Illustratio in horlum siccum Andreae Caesalpini. Florentiae, 1858.

Cialcara (P.), Storia naturale dell' isola di Ustica. Palermo, 1842.

Callet (P. M.), Glossaire vaudots, Lausanne, 1861.

Cameron (J.), Gaelic names of plants, Edinburgh, 1883.

Camsola, Flora astese. Asti, 185 i.

Camus (J.), Eludes de Lexicologie botanique, Additions an dictionnabre de. Liltré, (dans Rerue botanique, t. IIl, Toulouse, 1884).

Camus (J.), L'opera salernitana « circa instans 》 ed il testo primitivo del «Grant herbier en françoys» secondo due codici del secolo $X V$, conservati nella regia biblioleca estense, Modena, 1886.

Camus (J.), Réceptaire francais du XIV e siecle d'après un manuscrit de Turin. (dans Bull. de la Soc. syndic. des pharmaciens de la Côte-rl'Or, Dijon, 1892.)

Canus (J.), Les noms des plantes du Livre d'heures d'Inne de Breta!me, 1894. dans Journal de Bolanique, nos 19-23, 1894).

Canus (J.), Un manuscril namurois du XVe siècle (dans Rev'ue des lanyues romanes, 1895.)

Canus (J.), Studio di lessicografia botanica sopra alcune note manoscritte del sec. XVI in vernacolo veneto. R. Istitulo veneto, t. II, 1881).

Caurs (J.) el 0. PExzif, Illustrazione del ducale erbario estense conservato nel R. Archivio di Stato in Modena. Dans Atli delli Societi dei nuturalisti di Iodena, Serie III, vol. IV, 1885).

Capello (L.), Dictionnaire piemontais-français, Turin, 1814.

Cariot, Etude des fleur's, Lyon, 1865.

Casaccia (G.), Dizionario genovese ilaliano, 1873-1875.

Castor (J. J.), L'interprète provencal, Apt, 1843, in-12.

Chamberlain, Glossary of West Worcestershire words, London, 1882.

Сhambure (E. DE), Glossaire du Morvan, 1878.

Cherubini (Fr.), Vocabolario mantovano, Milano, 1827.

Cihac (A. DE), Dictionnaire d'étymologie daco-romane, 1870.

Clédat (L.), Le patois de Coligny (Ain), dans Revue des palous, 1887.)

Cockayne (0.), Leechdoms, wortcunning and starcraft of early England, 1866, 3 vol. in-8.

Colignon (E.), Flore d'Apt (dans Annales de la Soc. littér. d'Apt (Vaucluse). 1864-1865.)

Colda (Aloysio), Herbarium pedemontanum, Turin, 1837.

Colmeiro (M.), Diccionario de los diversos nombres vulgares de muchas plantas. Madrid, 1871.

Comelin (Cisparus), Flora malabarica, Lngduni Batav., 1696. 
Comolu, Flora comense, 1834-1836.

Companyo (L.), Histoire natur. des Pyrénées orientales, Perpignan, 1864.

Contejean (Сн.), Glossaire du patois de Montbéliard. 1876.

Conbichon, Le Propriétaire des choses très utiles, 1525.

Corblet, Glossaire du patois picard, 1851.

Cordus (E.), Botanologicon, Coloniae, 1533, in-12.

Cornu, Phonologie du Bagnard, (dans Romania, 1877:.

Coronedi-Berti, locabolario bolognese, 1869-1874.

Costa (A. C.), Introduccion à la Flora de Cataluña, Barcelona, 186.4.

Cotgrave, A french and english dictionary. Editions de 1650 et de 1660.

Couverchel, Traité des fruits ou dictionnaire carpologique, 1839.

Coczıxié, Dictionnaire de la lanyue romano-castraise, Castres, 1850.

Cregeen (A.), Dictionary of the manks language. Douglas, 183.).

Crouan, Florule du Finistère, Brest, 1867.

Cuxi y Martoreld, Flor de Calella, dans Anuari de lı Assoccincio l'excursions catalema, 1883.

Cupani (Fr.), Ilortus catholicus, Neapoli, 1696-1697.

Cuveiro Piñol (J.), Diccionario yallego, Barcelona, 1876.

DANNEIL, Wörterbuch iler altmärlisch-plattleutschen Mundart, 1859.

Dirlingtox (Tu.), Folk Speech of South Cheshive, 1887.

DARToIs, Importance des patois (dans Acad. des sciences, belles lettres, etc. de Besancon, 1850.)

Dasnor, Dictionnaire wallon-français, 1858.

Davies (HeGH, Welsh botanology, London, 1813, in-8.

Daries J.), Antiquæ linguæ britamnicæ nunc vulgo dictæ wallicre dictionarimm. Londini, 1632, in-4o.

DEBEalx (0.), Cotal. des plantes du territoire de Boghar. 1859.

DE Bo (L. L.), Westilaamsch Idioticon. Gent, 1892, in-4º.

DE Bosco J. J. oe Maxlils). Luminare majus. Lugd., 1536, in fol.

I)ECAISNE, Catal. des plantes représentées dans le livre d'heures d'Anne de bretayne, in-fol. (à la suite du Lirre d'heures d'Anne de Bretagne publié par Curmer. (Voy. J. Cayus, Les noms des plantes du Livre d'heures.)

DE CoCK (A., Vollisyeneeskunde in Vlaanderen, Gent, 1891.

DEcorde, Dictionn. du patois du pays de Bray, 1859.

I) Gorter D.), Flora VII provinciarum Belyii foederati indigena. Harlemi, 1781.

De Gubernatis (1.), Mythologie des plantes, 1878.

Delboulle, Glossaire de la Vallée d'Yères, 1876.

Delicata, Flora melitensis, Melitae, 1853.

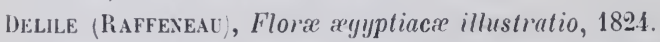

DEscemet (CH.), Ln erbario del secolo XII ossia nomenclatura ricatata dall' 
iynota lingua di Santa Ildegarda. Estratto de Memorie della Accal. dei unovi Linrei, Roma, 1884.

Des Etangs (S..) Noms pop. des plantes de l'A ube el des environs de I'rovins (dlans Mlém. de la Soc. d'ayric. de l'Aube, 1814.

Despontes, Flore de la Sarthe el de la Mayenne, 1838.

Desvaux (A. X., Flore de l'Anjou, 1827, in-8.

DiEfENBACH L.), Glossarium latino-yermanicum mediae et infimae latinitatis, 1851.

DODONAEus R. , Ilerbarills oft r'ruydt boeck, Leyde, 1608, in-folio.

Dodonaeus R., IIerbarms oft cruydl-boeck, Antwerpen, 1644, in-folio.

Dons b.), Cilnestomathy of musthii or afyhan language, 1847.

Dozs (R.), Supplément aux dictionnuires mrabes, Leyde, 1881.

Dozr et Engelmaxi, Glossoire des mots espaymols et portugais dérires de larabe, 1869.

DrugexdoRfF GEokg, Zur Folhsmedicin Turliestuns. (Tir. at part de Buchner's

N. Reperlorium, Band XXII, Heft 3, Dorpat, 1872.

Dubors el Travers, Glossaire du patois normand, Caen, 1856.

Duboul Axel, Las plantos as camps, Glossaire patois, Toulouse, 1890, (deuxième édition.)

Du Cange, Glossarium mediae el infimae latinitatis. Edition Didot.

Duchesse (L.), [L. à Quereu], In Ruellium de stippibus epitome. I'arisiis, 1544 , in-12.

Dukz (N.), Dictomnaire francois-nllemand-latin, Amsterdam, 1664, in-40.

Duez N.), Dictionnaire italien et francois. Genève, 1678, in-8.

Derrawo, Calentrier de la Flore alyérienne. dans Bull. de la Soc. climatol. alyér. 1865.)

Durhein (C. J.), Schweizerisches Pflanzen-Idiotikon. Bern. 1856, in-8.

Duval L.), L'enquête philologique de 1812 dans les arrondissements dilencon et de Mortayne. Alençon, 1890.

Deverrier (H.), Les Touaregs du Nord, Paris, 186 亿.

EANDI (G.), Statistica detla prorincia di Saluzzo, 1833.

E..roe (J.), Enylish Plant Numes from the tenth to the fifteenth century. Oxford, 1880, in-16.

Liber (P.) et G. Peucer, Ippellationes quudrupedum, insectornum, volucrum, piscium, fruyum leyuminum, olerum et fructuum communium. Se trouve à la suite de : Vocabula res mummariae ponilerum et mensurarum graecu et latima, etc. Vitebergae, 1556, in-12.

Elwortil F. T.), West Somerset worl-boul, Londun, 1886.

ERndetelius, Warsavia physice illustrata. Dresdae, 1730.

Escaller (E. A.), Rem. sur le patois suivies d'un rocabul. latin-français inérlit du X/Fe siccle. Douai, 1856. 
Estiense (Ch.), Dictionarium latinogallicum, Lutetiae, 1561, in-40.

Evans (D. Suliax). Diction. of the welsh language. Carmarthen, 1887.

Falk (J. P. , Begträge aur topogruphischen Kenntniss des russischen lieichs, Saint Pétersb., 1786.

Fisuen (J.), Flore populnire wallonne dans Bulletin de Follilore, Liege, $1891-95$.

felduax (J.), Imdex pluntarum in Lapponiu fennici lectarum. (dans Bull. de la Soc. imp. des naturalistes de Hoscou, 1835.)

léraro (G.), Glossario monferrino, Ferrara, 1881.

Ferraro G. , Botanica popolare di Carpeneto d'Acqui (dans Archirio d. Trad. pop. 1885.)

Figuera, Diccionari mallorqui-castella, Palma, 1840.

Fillassier, Dictionnaire du jarlinier francois, 1791.

Finanore (G.', Vocabolario dell' uso abrus:ese, Lanciano, 1880.

Fixamore (G.), Botunica popolare abruszese (dans Archirio d. Trad. pop). t. VIII, P. 99-46.)

Fleuri (J.), Essai sur le patois normand de La Hague, Paris, 1886.

Flëckiger and Haxisur, Pharmacogruphiu, London, 187.'.

FосKE (W. O.), Die volksthïmlichen Pflan:ennumen im Giebiete ler unteren Weser und Ems. Bremen, 1870.

FoLer 1).), English-irish Dictionary. Dublin, 18\%.

Говвг (R., Tocubulary of East-Amylia. Lond., 1830.

Fonı (11.), Dictionnaire liègeois-frunsuis, Liège, 1886.

lika Is, Synopsis plunturum florie classicie, 1870.

Frestag (7. II.), Lexicon arabico-latimum, 1830.

Faiexp (H.). Glossmy of Deronshire Plant-names, $188 .$.

Frusn (H.), Flowers and Flower Lore, London, 1886.

Finschimer (11.), Preussisches Würterbuch, 1882.

Fucus (L.), L'histoire des plantes, Lion, 1558.

Fuchsu's (L. , De historia stirpium, Parisiis, 1546.

Fusz. (I.), Alphabetarische Zuzummenstellun!! der sächsichen, unyarischen, walachischen und deutschen Trivialnamen in Siebenhüron wilduruchsender. oder cultivirter Pflanzen ilans Archiv. I. Ver. f. siebenbüry. Landesk.. 1. III, Hermannstadt, 18.18).

Gallé (I. H.), Sachsischen Namen van Plunten dans Tijilschr. voor nederlamlsche Taal-en Lettertunde, Leiden, 1887, p. 261-282).

G.ancix (E.), Non'eun dictionn. provensal, Draguignan, 1841.

G.Aridel, Hist. des plantes des environs d'Aix, 1716.

Gary, Dictionnaire patois du Tarn, Castres, 1815.

G.ITEr.u, Deser. des plantes des environs de Montumban. Montauban, 1789. 
Gattive (G.), Saygio sulla prohlu:ione spoutunea dell aygro materano. Napoli, 1892 , in-8\%.

Germano (G.), Vocabol. italiuno e greco volyare, Roma, 1622.

Gilliéron J.), Patois de Vionnaz (Bas Valais), 1880.

Goderrond, Dictiom!. Ie l'anc. Iangue frans., 1880 et suiv.

Grandgagnage et SiHel.er, Dictionn. étymol. de la langne wallonne, liege el Bruxelles, 1816-1880.

Grangier (L.), Glossaive firibouryeois, 1864-1868.

Grassmann, Deutsche Pflanzenuameu. 1870.

Graves, Catulogne des plantes de l'Oise, Beauvais, 1857.

GrÉgolre (P.), Jictionn. frantrois-celtique ou frantois-bieton, hennes, 1732. Grimi J. und W.), Deutsches Wörterbuch.

Griseb.ch, Flor'a of the british indian islamls, 18 (8i.).

Grister G.) Viridarium lusitanicum, Ilysipone, 1660.

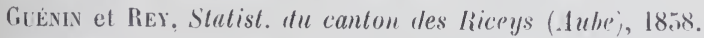

Gunbemin (J.), Glossaire des putois de la biresse chilonnuise (dans Mem. de la Soc. de Cihulon-sur-Saóne, 1852.

Gussone, Enum. plantar. insulie intrimes, Neap. 18.j.

Gusumpaur (T.), Focabol. botanico napoletano, Napoli, 1887.

Hallant N.), filore populaire des Vosyes, Epinal, 1886.

H.soteau et Letounnex, La Kabylie, 1872.

H.ınrs, Catal. des plantes du Var, Draguignan, 1853.

Histoeu (B. Petriceicu), Elymoloyicum maynum Rumunix, Bucuresci, 1887 et suiv.

HĖcart, Dictionnaire rouchi et fransais, Valenciennes, 1834.

Helureich (Th. voN), Die Nutzpflanzen Griechenlanls, Athen, 1862.

Hörer F. und M. KRONFELd, llie Vollismmmen der niederösterreichischen Pflanzen, Wien, 1889, in 8 .

HoHeNaCKer, Enum. plantar. provincize Talysch, 18:37.

Honvorat, Dictionn. movencal français, Digne, 1846.

illoet, Dictionurie. London, 1572.

Hulsius (L., Dicl. frangois-allemand-ilutien, 1616, in-1º.

HtPEL 1. W.), Idiotikon der deutsche Syrachen in Lief- Ind Eihstlumd (dans

Veue nordische Viscelluneen, XI, XII, 1795 et XVII, 1797, Riga.)

lb.N-EL-Bë̈thar, Traite des simples, trad. par L. Leclerc, 1877-1883, in-4º. JaCkson, Shropshire Woord-book, 1879.

Jасовт, Litauische P'flunzennamen (dans Mitteilungen der litanischen litter Ges?llschaft, 11, 3, 1884).

Jamieson (J.), Etymoloy. dictionary of the scottish linguaye, 1879-1882.

Jalbert, Glossaire tu centre de la France, 1864-1869.

JExixis (J.), On some of the dialects of Euylumal, 1825. 
Jenssex-Tusch (H.), Nordistie plantenaine, lijobenhavn, 1867, in-8`.

Joxisx (P.), Dictionn. du patois saintongeais, Royan. 1869.

Jorer (Ch.), Flore populaire de la Vormandie, Caen, 1887.

Jossier S.), Dictionnaire du patois de l'Yonne, Iuxerre, 188..

Jourdax (P.). Flore murale de Tlemcen. Alger, 1866.

Jocrdax (P.). Flore murale du tombeau de la Linetienne, 1867.

lorce P. W.), The origin und history of irish names of places. Dublin, $1869-1875$

Kelly J.), A manx and english dictionary, Douglas, 1866.

Keogh (J.), Bolunologia hibernicu. Cork, 1735 , in-4.

hirschleger, Flore d'.1lsace, Strasb.. 18 jo.

liobbaxi (P.), Gifthistorie des Pflanzenreichs, Wien, 1807.

LouTz. Flore du Grand Duché de Luxembonry. 1873.

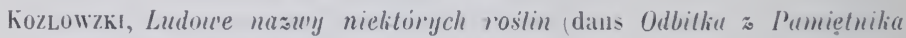
Fisyjoyrafic:ulleyo, 1885.)

Labotderie, Гocub. du palois de la IIaute-.1uvergne dans Mem. de lu Soc. (les Intiq., 1836.)

Labothasse, Glossaire du patois de la lleuse, 1887.

L.ACAVALLERI.1. Ga:ophylacium catalano-latimm, 1696.

L.AFFage A.). Toms patois des animur et des plantes dans les environs de liurcassonne (dans Bull. de la Soc. d'Etules scientifiques de l'.1ude, 1. 11, 1893.

L.sciddetc J.), Le Cutholicon, Diclionnaire brelon, frantruis et latin (1499) publie par LE ME., Lorient. 18:2.

L.dirèz-Fossat. Flore de Tarn-el-Goronne, 1847.

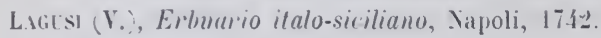

L.IL.INxE, Glossicire dı patois poilevin, 186i.

L.M.ARck (DE) et DE C.MDolle, Flor'e frunçaise, 1805 et années suir.

Laxdors (H.), Die westfälischen Pfanzennamen (dans Botanisches lientrull.latt, 1882.)

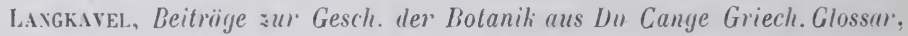
Berlin, 1866. Proyramm des Friedr.-Werlerschen Gymnusiums .

L'Armene]. L'A**, Dictionn. francois-breton du dialecte de l'annes. A Leide, 1741 par Cillart de Kiérampoul.

Laterrade, Flore borleluise, 1816 et autres éditions.

Lalgier de Chartrolse, Nomenclat. patoise des plantes les entirons d'Alles, Irles, 1838.

LfCEsNe (Ed.), Le patois artésien dans Mém. de l'Acad. des sciences, etc. l'Arras, ge série, t. XX, 1889).

Leclerc (L.), Traité des simples, par Ilon el Beilhar (auteur arabe mort en 1218), Traduction, 18:i-18\%3. 
LEDIEL . 1.), Monographie d'un bourg picard 'contient un Glossaire du patois de Démuin, Paris, 1893.

LE Goxidec, Dictionn. breton-franrais, Saint-Brieuc, 1850; Dictionn. francais-breton, Saint-Brieuc. 1847.

Legraid (A.), Statistique botanique du Fore:. 1873.

LEdeuxe, Flore des environs de Spa, 1811.

Lenticchis, Flore du Tessin, Lugano, 1884.

Le l'elletier, Dictionn. de la langue bretome, Paris, 175.'.

Lépixis (G. Godin DE), Noms palois des plantes de la Corréze, Auch, 1886.

Lespy et R.IYMoxb, Dictionnaire bearnais, Montpellier, 1887.

LetaCQ, Noms ruly. des plantes usités duns les cantons de Vimoutier's et de La Ferté-Fresnel, Argentan, 1888, in-8.

LETACQ, Flore popul. des environs d'.1lencon et de Carrouges Urne) (dims Annunire normand, Caen, 1895, p. 989-305.

LEXER M.), Kämutisches Würterbuch, 186ュ, in $4^{n}$.

LELA.1CK, Dict. des noms wallons des envir. lle Spa, Liege, 1884.

L'Herminier, Synonymique créole et botanique des arbres et bois indigenes el exotiques observes a La Guadeloupe (publice a la suite de Notice sur. F.-L. L'Herminier, par G. Guibourt, Paris, 183i. in- $8^{\circ}$.

Likgard (1.), Flore de Bretagne, Paris. 1879, ill-80.

LightFoot (J.). Flora scotica, London, 1777.

LiNDE, Slownick jezyka polskiego, Lemberg, 1857.

Linocier, Ilistoire des plantes, Paris, 1584, in-8\%.

L.ıxs, Ess. s. les v'égétau.r utiles des Bouches-du-Rhine, 186:3.

LoIseletr-DEsloxgCHayps, Manuel des plantes usuelles, 1808.

LONG, Hict. of the Isle of Wight dialect, 1886.

Loret et Barrandox, Flore de Montpellier, 1876.

LORITzA, Neues Idiotilion viennense, 1847.

LonRaix (D.), Glossaire du patois lomain, 1876.

Mac Alpixe, Gaelic Dictionary, Edinburghlı, 1858.

Macaleso Storaci, Iuovo rocabolario siciliano, $18 \mathrm{i}$.

MaIL (Ти.), Tíat. sur les noms palois des plantes cultivees dans bull. de la

Soc. prat. d'hoiticultuie de l'arr. d' letol, 1883-188i).

Malaspixi, rocabol. parmigiano-itnliano. 1859.

MaLlouf, Dictiomaire francuis-turc, 1881.

Malval, Dialectes de la Basse-Aurergne, Clermont, I878.

Maxfredi, Dizionario puvese-italiano, 1874.

Ilaréchal (V.), Flore légendaire de Bretagne, Saint-Brieuc, 1884.

Marès et Vigrneix, Cat. des plantes des Baléares, 1880.

M.nigot (H.), Flove de l'ile de Znnte, Genive, 18 il.

Martexis (Vos, limise nurhe lenediy. I lin. I82y. 
Maulny, Plantes des environs du Mins, Avignon, 1786.

Meddyyon Myddfui, Llandovery, 1861, in-8 ${ }^{\circ}$.

MELCHon, Vocabolario bresciano, 1817-1820.

Méxı̇̇e (СH.), Glossaire anyevin, Angers, 1880.

Mesiski, Thesaurus lingum orientalium, Vienne, 1681-1687, in-folio.

Mentzel, (СН.), Lexicon plantarum polyylollon, Berlin. 1715.

Métivier (DE), De l'agriculture des Landes, Bordeaux, 1839.

MÉTwiek (G.), Dictionnaire franco-normaml, Londres, 1870.

IEexer (Enxst H. F.), Geschichle der Botanik, Königsberg', 1856.

Meter (L. E.), Glossaire de l'Lunis, 1870.

MEXER (PAUL), Jeurième rupport sur une mission litt. (dans Irehires des missions, 1867.)

Mikosich, Die slut. Elem. in magyar., Wien, 188.1.

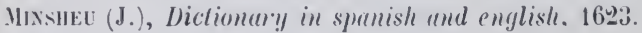

Mistral (F.), Lou Tresor dou Felibriyje.

IloLARo, Le mantais langaye corrige, Lyon, 1810.

Moxtelro de Carvalho, Jice. portug. das plantus, 1765.

Movtesson C. R. bE', Tocub. du Mlunt-Maine, 1859.

Moxtı (1'.), Vocubol. dei dialelli di Como, Milano. 18.15-1856.

Mongax J.), Flora of Llanbrynmair (dans Bye-Gones, 18!12, p. 143-452).

Mons (J. H.), filora sardon, Taurini, 1\$3̈-1859.

MonRI (A.), Vocubolurio romurynolo, Faenza, 1840.

Mortillaro (V.), Nuovo Disionario siciliano, Palerme; 1853.

Mocrier, Glossuire patois des végpitaux du Dauphiné jans Bull. de lu Soc. d'Archieol. de la Diome, Valence, 1889-1890).

IowAт J. L. G.', Alphita, a medico-botanical ylossary from bodleian manuscript, 0xford, $188 \mathrm{i}$, in-4 ${ }^{\circ}$.

MïLlèn (1.), Alphab. Wörterb. lateinischer, deutscher und böhmischer Namen der Pfanzen, Prag, in-4º oblong, 1866.

Muxil (G.), Noms arubes des plantes de l'Alyérie (dans bull. de lı Soc. botun. de Fr., t. XIII, 1866, p. 217 et suiv.)

Iixisis (l\%), Vocabolurio ferrarese, 1705.

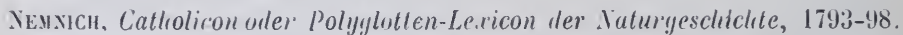

Fompruclutor communium rerum... Iuctore Adriano Iunio Medico... En celte derniere élition "esté adionstèe la langue brelonne... [par] Maistre Gvillaume Qviquier de Ros [coff]. Morlaix, 1633.

Nomi adoperuti in Italia a designare le piante di bosco (dans Annali del ministero di ayricolt. Firenze, 1873!.

Notice sur les forêts de la Tunisie et catal. des coll. ì l'Exposition de 1889, Tunis, 1889.

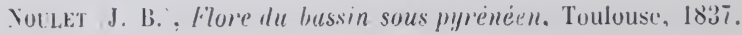


Oberila (H. G.). Description du Ban de la Roche, Strasb., 1806.

OLivier (E.), Flore popul. de l'Allier, Moulins, 1886.

OLbiuer, Cat. de la Flore de Porquerolles, Marseille, 1885.

Onarn (1D,), Glossaire pat. d'Ille-et-Vilaine, 1886.

Oribignу (D'), Dictionn. d'hist. natur., 1841.

O'Renly (ED.), Irish-English Dictionary. Duhlin, 1817.

Oudenans, De Flora van Nederland, Amsterdam, 1872.

PAGÈs (1.j, Cot' de floütos, poesios, Paris. s. d.

Palkowitsch, Böhmisches Wörterbuch. Pressburg, 1821.

Palmireno L.), Vocabulario del humanista. Barcelona, 1575.

PaLUx II.), Catal. des plantes du territoire d'Avignon, 1867.

PARIS (E. G.), Vingt-deux mois dans le Sahara algerien dans Bull. de lu

Soc. bot. de Fr. 1866$)$.

Pasurale: G. A. , Fora vesuriana. Napoli, 1869.

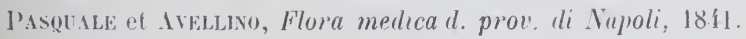

Patuut, Plantes méducin. des envir. de Toulon. Toulon, 1864.

Patriarch (G.), Vocabol. venes. e padovano, Padova, 1821.

l'Etagia L.), Viaggio in alc. luoghi d. Basilicata e d. Calabria citeriore.

Napoli, 1827, in-80.

Phimaon (Floriax, Vocabul. francais-arabe, Paris, 1860.

Puxaeus A.), Ilistorin planturum, Lugduni, 1561, in-18.

PINET 1. Du), Commentaire de P. Mathiolle sur Dioscoride, trad. par I. lu Pinet, Lyon, 1660.

PIPER P.), Iltdeutsche Pflanzennamen (dans Germaniu, 1881).

Pinox.l, Vocubolario friulano, 1871.

Pluncet, Contes, prejugés, patois de l'arr. de Bayeur, liouen, 183.1.

Poetevin (Fr. L.), Nouv. dictionn. francais-allemand, Basle, 1751.

Polak (J. E.), Persien. Das Land u. s. Bewohner, 1865.

Pollin (C.) Florll veronensis, Verona, 1824.

Puxt, Oriy. du patois de la Tarentaise, 187๘.

Porru V.), Dizionaru sardu-italianu, 1866.

Portus (sin.), Dictionarium latinum graco-burbarum, Paris, 16:35, in-10.

Poulet F. V.), Vocabul. du putois de Plancher-les-Mines Haute-Saòne, 1878.

Poumaréde, Termes usuels, Toulouse, 1860 .

Pouzolz, Flore du Gurd, Nìmes, 185̃-186\%.

Prax, Algérie et Tunisie, Règne végétul (daus Revue de l'Orient, t. VIII, 1850 , p. $278-285$ et 342-348.

P'R10R, Popul, names of bristish plants, 1870.

Pirtzel (G.) und C. Jessen, Die deutschen Vollisnamen der Pflunzen.

Hannover, 188\%.

PLEL A.). Culalogue des plantes du Lot, 181́̃. 
Putspelu (N. DU), Dict. élymol. du patois lyonnais, 1887-1890.

P'vorı (B.), Vocabolario napoletano, Napoli, 1850.

Quedexreld (.I.), Nahrungs-, Rei:- und liosmetische Miltel bei den Maroklianer'n (dans Verhandl. 1. Berl. Gesellsch.f. Anthropoloyie, Berlin, 1887).

R.ATo de Irgüelles, Vocubulario de las palabrus que se hablan en el principado de Asturias, Madril, 1891, in-8॰.

Rallix, Descr. phys. de l'ile de Crète, Bordeaux, 1859.

R.Irocaro, Lexique roman, 1838.

lie (G. Fer.), Flora torinese, Torino, 18:5.

RE (G. Fer.), Flor'r segusiensis. Taurini, 1805.

Regus (II.), Nomenclat. franco-provencale des plantes (lans Mem. de l'Acar. des sciences d'Aix, t. XI, 1878.

Resssch, Maitre Audré de Coutances (dans Arch. f. dhs Silud. 1. nener. sprach. ron Herrig. 1880); 1. 170 at p. 176 se trouve nn chapitre intit. secrets de médecine.

licharus (W.), Welsh-Enylish pocket Dict., Wrexham, s. d.

Robix, Le Prérost, Passi et Blosserille, Dict. du patois de l'Eure, Evreux, 1889.

Rosenthal (D. A.), Synopsis plantarum diaphoricarum, Erlangen, 186 i.

Rotcer. (F.), Flore du Nord de la France, 1803.

Rotger (F. A.). Topogrr. statist. Iu cunton du Viyan (Gard, Montpellier. 1819.

Rousser (Сн. , Gloss. du parler de Bournois Doubs), 1894.

Rover CH.), Flore de ln Cóle-rl'Or, 1881.

Rozier, Cour's d'ayriculture, 1793 et années suiv., $12 \mathrm{rol}$. in-4ª

Sicaze (J.), Flore popul. de Luchon (dans Rev. des Pyrénées, 1890).

S.ccindo, Flora trecigiana, Venezia, 1864, in-8.

SAlvages (DE), Dictionnaire languelocien, Alais, 1820.

scoxxagatt, Erbe de' prati asciulti (dans Atti della soc. patriotica di Miluno, t. III, 179', in-1\%.

scmiмвасн, Würt. d. niederd. Mund. de Fïrstenth. Güttingen u. Grubenhaygen, 1858.

Schyalhalsex, Flor jugo-zopalnö Rossil (La Flore de la Russie du sudonesl. Kiew, 1886.

SCHMeler (J. A.), Bayerisches Wörlerbuch, München, 1872-1878.

SCHMeller (J. A.), Cimbrisches Wirterbuch, Wien, 1855.

Scнmі J. Cнr.), Schü̈̈bisches Wärterbuch, Stuttgart, 1831.

Sснирт Мах), Ein botanischer Garten in Meissen im. 16 Jahrhundert dans Mitleilungen des Ver. f. Gesch. 1. Stault Meissen, 1895, 1. 45-70).

Schlexberg (Vos. Mendisches Vollisthum. Burlin, 1882. 
Scuderi (S.), Tratt. dei boschi dell' Etna (daus Atti dell' Accad. yioenia di scienze nat. di Catania, t. I, 1825).

Semertier (Сh.), Vocab. de l'apothicaire-pharmacien, Liège, 1891.

SiвthoRP, Florae græcæ prorlromus, London, 1806, in-8.

Sigart (J.), Glossaire montois, Bruxelles, 1866.

Somarera, Tesoro d. lingua grece iolyare, Parigi, 1709.

Soubix: (A.), (iat. des plantes du cant. de Saint-Germain dans Bull. de la Soc. des Etudes litt. du Lot, 1884 ).

Spaxo, Vocabolario sardo italiano, Cagliari, 1851.

STAlder, Ver's, ein's Schweizerischen lliotikon, Aaran, 1812.

Stender, Lettisches Lexicon, Mittad, 1789, in-80.

Strpilanus (R.), l)e lutinis et graecis nominibus arborum, etr. cum yallica eorum nominum apprellatione, Lutetix, 1547, in-16.

Steven Chr. Vox), Verะ. d. auf d. taurische Ilalbinsel wildwuchs. Pflanzen (dans Bull, de la $S$. des natur. de .Moscou, 1856).

Stokes (Wh.), Cormac's Glossrury. Calcutta, 1868, in-4.0.

Stokes (Wh.), Anecdota oxoniensia, medieval and modern series, Part $V$. 0xford, 1890.

Stolze und Andreas, Die Ilandelsverhältaisse Persiens (dans Petermann $s$ Mutteilunyen, Ergünzungsheft 77, Gotha, 188i).

Storrie (J.), The Flor'a of Cardiff. Cardiff, 1886.

ŠLLK (B.), Jugoslavensti imenik bilja. Agranı, 1879.

Targioni Tozzetti, Dizionario bolanico ital. Firenze, 1825.

Тсннатснеғ, Asie mineure (Trois. part. : Botanique), 1860.

Temrinck (Is.), Plantlore. I. De plant een levend, beжield, handelend w'esen. Gent, 1892.

Thibault (A.), Glossaire du Palys blésois, Blois, 189!2.

тнiniat, La vallée de Cleurie, Mirecourt, 1869.

Threlkeld, Synopsis stirpium hibernicarum, Dublin, 1797.

Tissot (J.), I'utois des Fourys (I)oubs), Besancou, 1865.

Tournon, Flore de Toulouse, 1811, in-80.

Treichel (A.), Polnisch-ivestpreussiche Vulyärnamen von Pflanzen (dans Schrift. 1. naturforsch. Gesellsch. zu Danzig. V. Band).

Treiches. (1.), Volksthümliches aus der Pflanzenwelt, besonder's für IVestpreussen (dans Schriften der naturforsch. Gesellschaft :u Dan:ig, Bd. V. VI et dans Altpreussische Monatschift, Bd. XXIV u. folg.)

Troude, Nouv. dictionnaire francais-breton, 1869 ; Noun. dictionnaire pratique breton-francais, Brest, 1876.

UlMaN, Lettisches Wiirterbuch, Riga, 1872-1880.

Val.l.ddares Nuñez, Jiccionario gallego-castellano. Santiago, 1881.

Valdottox-Aubert, Vallorbes. Esquisse statistique. Lausanne, 1875. 
Vax Dale J. H., , „ieuw Woordenboek der Nederlandsche Taal, 1881.

Varreda y Vila, Catcilech de la Flara de la Vall de Nuria. Barcelona, 1882. Varssier, Dictionn. patois francais de l'Aveyron, Rodez, 18i9.

Vicat, Hist. des plantes vénéneuses de la Suisse, in-8`, 1776.

Victor (J.), Tesoro de las tres lenguas francesa, italiana y espaùola. fonì;, 1609.

Vigutier (A. L. G.), Notice sur la ville d'Auduze (Gard), 1823.

Villeneuve (ve), Stutistique des Bouches-du-Rhône, Marseille, 1821.

Vunonis, Fleurs de pleine terre, 1870.

Visathey (Fr.), Grödlen, der Grödner u. s. Sprache, Bozen, 1864.

lincent et Bacues, Topographie de Nimes et de sa banlieue, Nìmes, 180".

Walters (J.), English-Welsh Dictionary. London, 1794.

Wirtmaxx (B.), St. Gallischen Volksbotank, St-Gallen, 18;i.

Wirson, Index to the natue names of indian plants, 1868.

Wen et Berthelot, Hist. naturelle des Canaries, in-4º

Whiner (Josef), Lezeburjesch-latein-fransesch-deitschen Dirionèr fun de Mlanzen, Luxemburg, in-8' de 102 p.

Weinhold, Beiträge zu einem schlesischen Wörterbuche, 1855.

WF.Tz, Die aachener Mandart, 1836.

West, Beytr. aur Beschreib. von St. Croix, etc., Kopenhagen, 1791.

WiedfaAx, Ehstnisch-deutsch Wörterluch, 1869.

Wilitays (J.), Fuunula grustensis. Llanrwst, 1830.

Hilliom und Lange, Prodromus florae hispanicae, 1877.

Wirtgen, Die leggetation der Eifel (dans Verh. d. naturh. Ver. der preuss. Rheinlanile $u$. Westph. 1865.)

7.aldi (C.), Dizionario piemontese, 1830.

Z.ANetti (Z.), La medicina delle nostre domne, Citti di Castello, 1892, in-12. ZELIGzoN LÉOx), Lothringische Mundarten, Metz, 1889.

ZErsi (E.), Piante rascolari della provincia de Brescia, 1871.

Zwaxziger, Veræ. d. in Kürnten volksth. deutschen Pflanzennamen, 1888. 


\title{
TABLE DES MATIERES
}

\author{
10 FAMILLES.
}

\begin{tabular}{|c|c|c|}
\hline Renonculacées & 1 & Xyımphéacées. \\
\hline Magniolacées. & 131 & Papavéracées. \\
\hline Anonacées & 132 & Fumarices. \\
\hline Menispermíes & 135 & Crucifères . \\
\hline Berbéridées & 137 & \\
\hline
\end{tabular}

2o GENRES ET ESPEGES ( Noms latins,

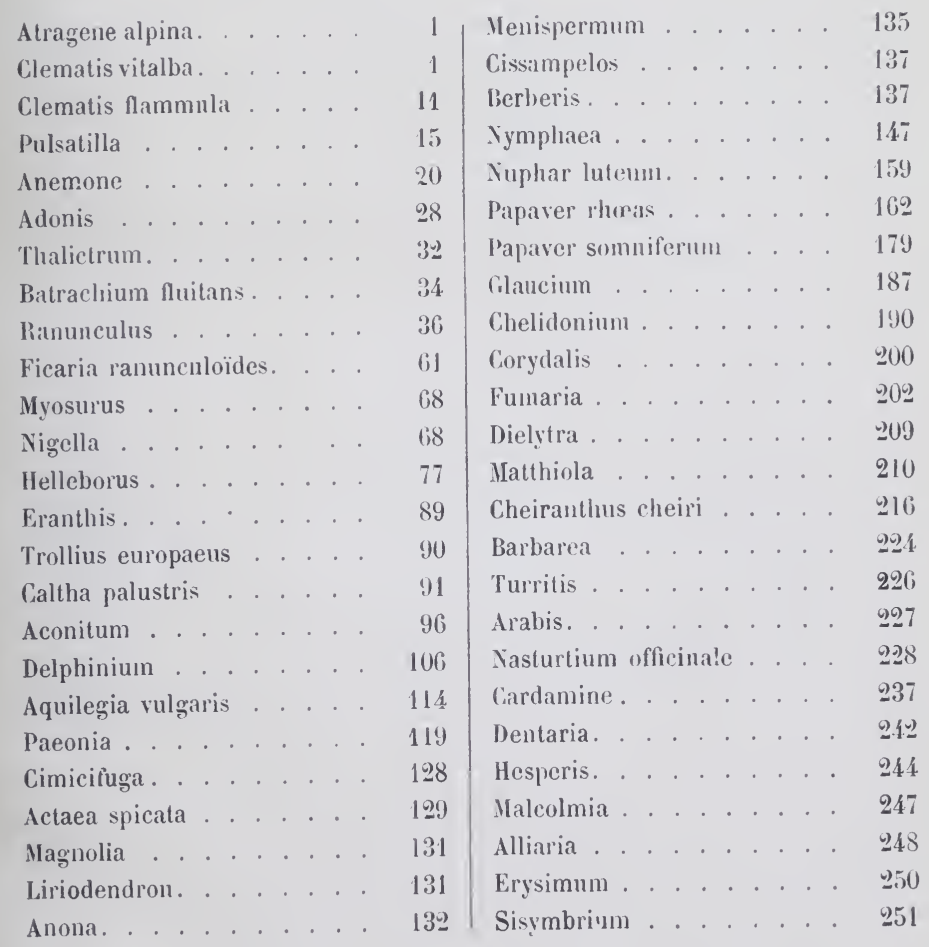


30 GENRES ET ESPLCES (Noms franerais).

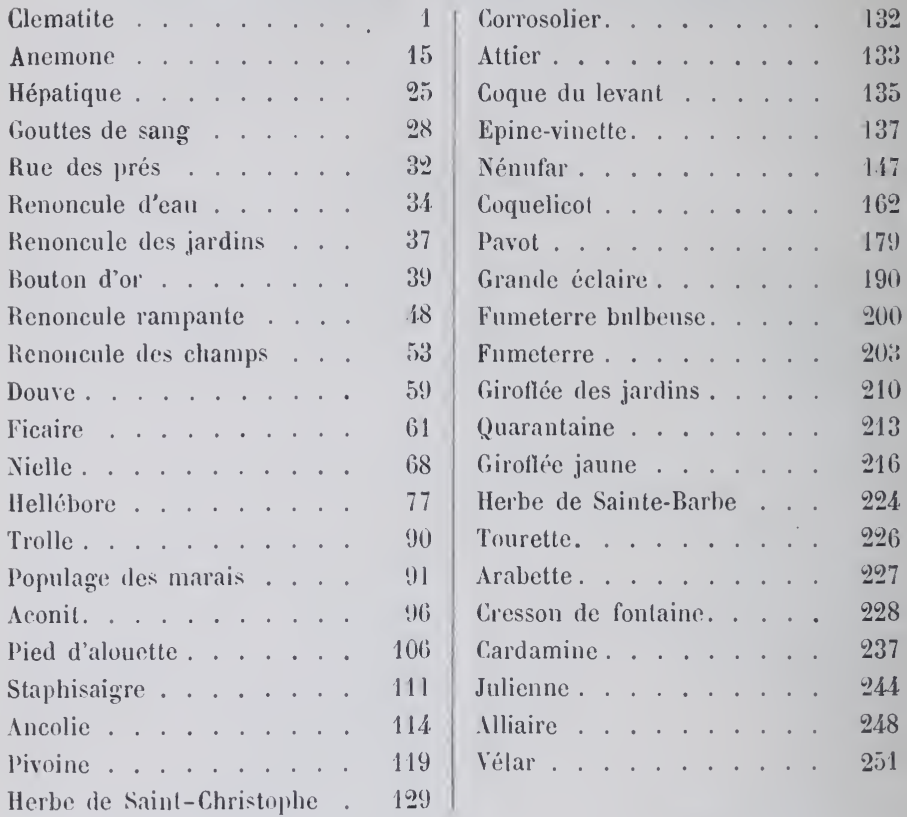

\section{ERRATA}

Page 64, 16 ligne, au lieu de Bolla, lisez Colla.

P. 100, 32e ligne, au lieu de blysiau lisez llysiau.

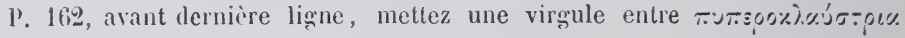

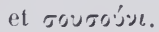

P. 170, ligne 4, au lieu de T. Forx lisez V. Forx.

P. 171, ligne 5, mettez une virgule entre done et donëtte.

P. 171, ligne 5, an lieu de Cunio lisez Cun o.

P. 174, la note (2 appartient au mot row-moc'h deux lignes plus haut. 


\title{
DU MÊME AUTEUR
}

\author{
EN VENTE \\ A L A
}

\section{LIBRAIRIE MAISONNEUVE}

Rue Madame, 26

Faune populaire, $1877^{-1883,6}$ vol., in- $8^{\circ} \ldots .49 \mathrm{fr}$.

\section{ON VEND SÉPARÉMENT:}

Tome I. Les Mammiferes sauvages de la France, 1877 . $\mathrm{xv}$ et 179 pp. ......... 5 fr.

Tome II. Les Oiseaux sauvages, 1879 . xv et 42 I pp. $\quad$ Io fr.

Tome III. Les Reptiles, les Poissons, les Mollusques, les Crustacés et les Insectes, I88I. xv et 365 pF. . Io fr.

Tome IV. Les Mammifères domestiques (première partie), I88i. xir et 276 pp. . . . . . . 8 fr.

Tome V. Les Mammifères domestiques (deuxième partie), I882. vi et 265 pp. . . . . . . . 8 fr.

Tome VI. Les Oiseaux domestiques et la Fauconnerie, I883. xir et 244 pp. . . . . . . . 8 fr. 
(20)

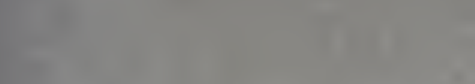

s.

$x^{2}+x^{2}$

a

$$
\text { 1. }
$$

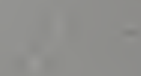

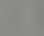

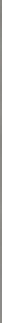

y.

$\sqrt{2}$

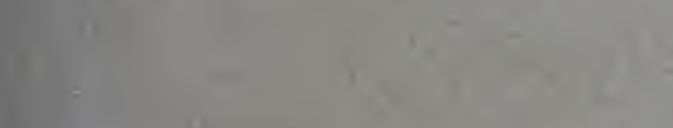
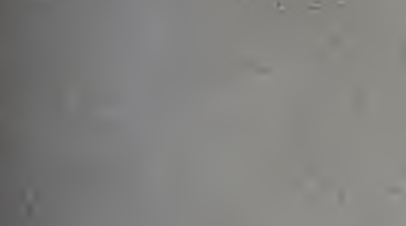

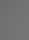

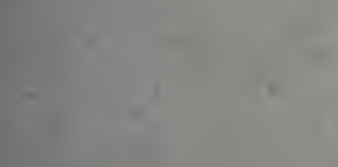<smiles>CCC</smiles>

+10

1)

$1=$

58

18.

$2=$

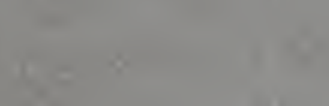




$x^{2}=1+3$

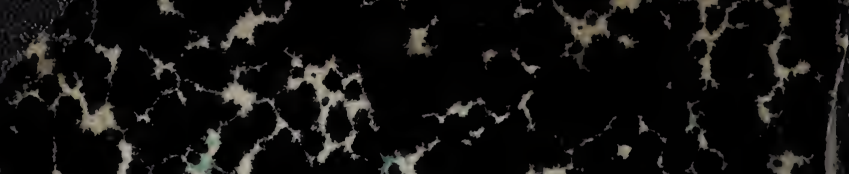
$x+2400$ 4. a 1 the $0+2 x+4+2$ $+x+5$

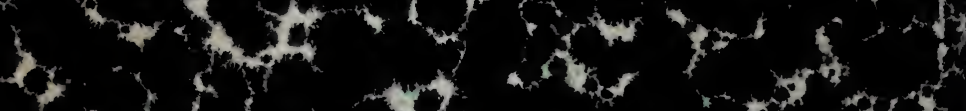
$x^{2}+x^{2}$ - 5 $+2 x^{2}+\cdots+1$

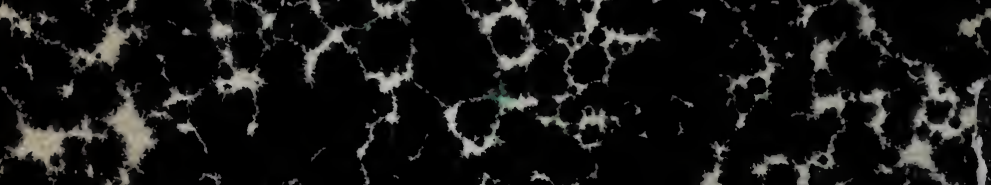

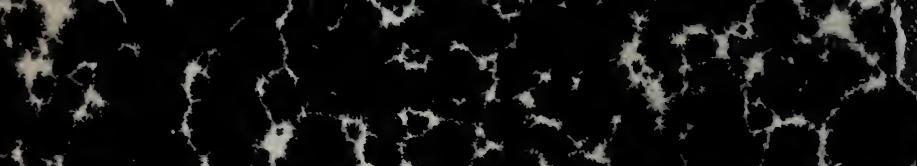
$4+2+30+2$

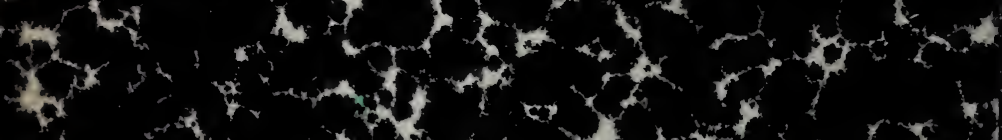
$x-3+y+7$ $x+2)+c^{2}$ $+x+3 y^{2}$

$$
\text { t }-4
$$

net $x^{2}+1$ $\left(x+4 x^{2}+x^{2}\right.$ $+x^{x}+x^{2}+a^{2}$

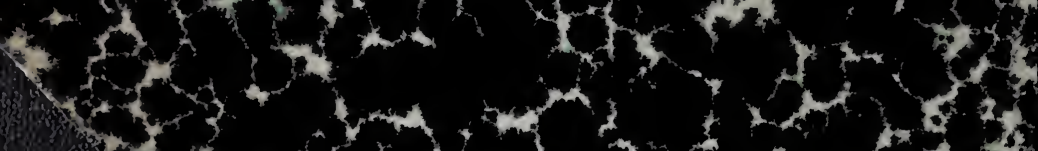

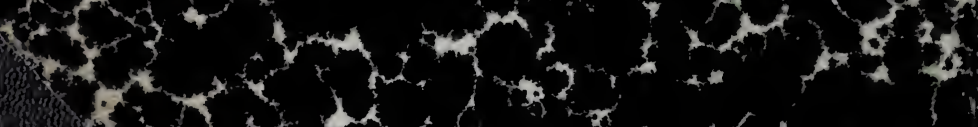

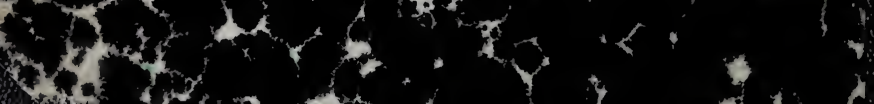
$+2 x+1+4$ $+4+4$ 